\title{
We are probably Wales' best kept secret": An Exploration of the Role of Care \& Repair Cymru Caseworkers in Facilitating Independent Living for Older People in Wales
}

\author{
Wolton, Joanna L.
}

How to cite:

Wolton, Joanna L. (2018) We are probably Wales' best kept secret”: An Exploration of the Role of Care \& Repair Cymru Caseworkers in Facilitating Independent Living for Older People in Wales. Doctoral thesis, Swansea University.

http://cronfa.swan.ac.uk/Record/cronfa49017

Use policy:

This item is brought to you by Swansea University. Any person downloading material is agreeing to abide by the terms of the repository licence: copies of full text items may be used or reproduced in any format or medium, without prior permission for personal research or study, educational or non-commercial purposes only. The copyright for any work remains with the original author unless otherwise specified. The full-text must not be sold in any format or medium without the formal permission of the copyright holder. Permission for multiple reproductions should be obtained from the original author.

Authors are personally responsible for adhering to copyright and publisher restrictions when uploading content to the repository.

Please link to the metadata record in the Swansea University repository, Cronfa (link given in the citation reference above.) 


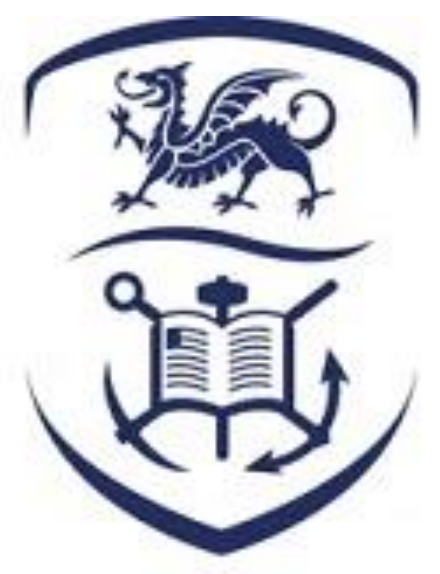

\section{Swansea University Prifysgol Abertawe}

\section{"We are probably Wales' best kept secret": An Exploration of the Role of Care \& Repair Cymru Caseworkers in Facilitating Independent Living for Older People in Wales.}

\section{Joanna L Wolton}

Submitted to Swansea University in Fulfilment of the Requirements for the Degree of Doctor of Philosophy 


\section{Summary}

Wales has an increasing number of older people, who typically have complex healthcare needs, and a diminishing pool of family members available to provide informal care. Care \& Repair Cymru are funded by Welsh Government to facilitate independent living at home and their Caseworker service plays an important role in achieving this. However, to date there have been no empirical studies that have examined the individuals who deliver the Caseworker service. The rationale underpinning this research is to fill a knowledge gap about how Caseworkers are acting as link agents to facilitate independent living for older people ageing in place in Wales. It is grounded in a policy context of welfare retrenchment and the promotion of partnership working.

An exploratory sequential mixed methods approach was adopted to examine the patterns of interactions between Caseworkers across Care \& Repair Cymru agencies in Wales, their professional links with external organisations and the relationships they develop with their clients. Data was gathered from four study populations using self-completion questionnaires, individual face-to-face interviews and focus groups. Social network analysis, bivariate statistical analyses and thematic analysis were used to map and interpret data.

In sum, the thesis found that Caseworkers represent a highly specialised workforce, playing a key role as link agents in facilitating independent living for older people who live at home. As an interpretive framework, social capital, human capital and emotion work theories help us to understand the sum of competencies that Caseworkers have developed and draw upon to work collaboratively in achieving this objective. Findings show that the context in which Caseworkers are operating (including agency location and certain issues encountered as part of delivering services) influences how they link with others to deliver services. 


\section{Declaration and Statements}

This work has not previously been accepted in substance for any degree and is not being concurrently submitted for any degree.

Signed (candidate)

Date

\section{STATEMENT 1}

This thesis is the result of my own investigation, except where otherwise stated. Where correction services have been used, the extent and nature of the correction is clearly marked in footnote(s).

Other sources are acknowledged by footnotes giving explicit references. A bibliography is appended.

Signed (candidate)

Date

\section{STATEMENT 2}

I hereby give consent for my thesis, if accepted, to be available for photocopying and for inter-library loan, and for the title and summary to be made available to outside organisations.

Signed (candidate)

Date 


\section{Contents}

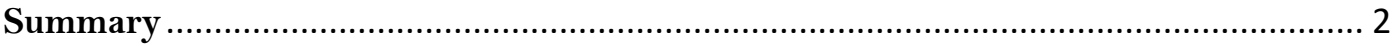

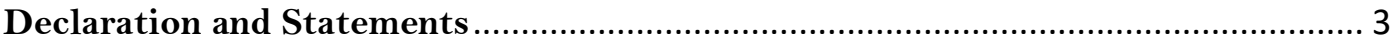

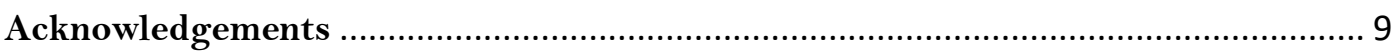

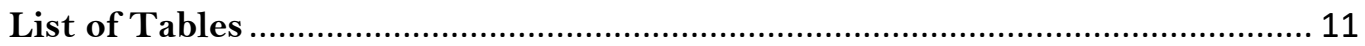

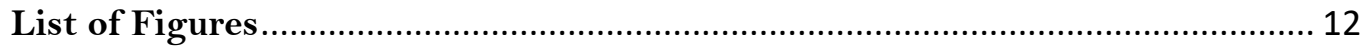

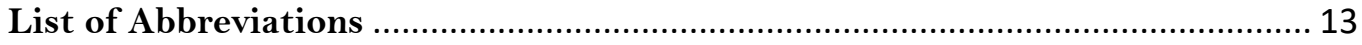

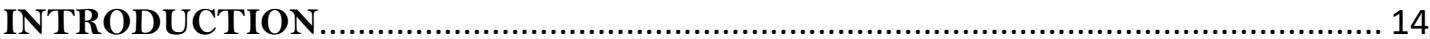

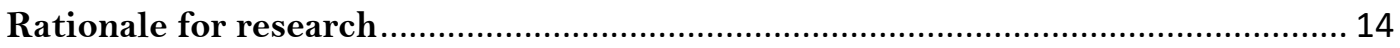

Mapping and explaining Caseworkers as link agents........................................ 19

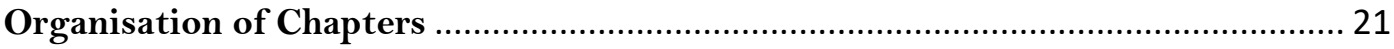

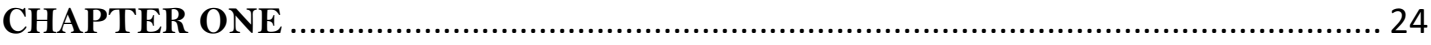

The Policy, Social, Cultural and Demographic Context in Wales .......................... 24

Policy response to an ageing population in Wales ............................................... 24

Defining Ageing in Place and Independent Living .................................... 25

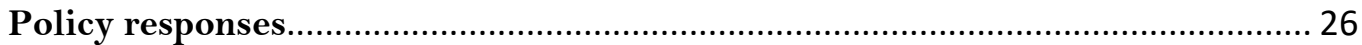

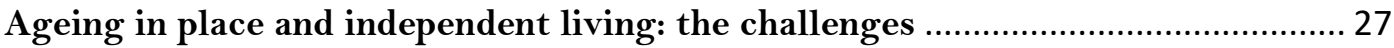

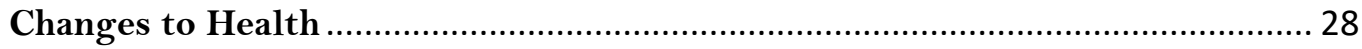

Changes to Family Roles ....................................................................... 30

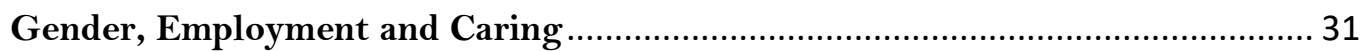

Housing in later life ............................................................................ 32

Care \& Repair Cymru: A Response to an Ageing Population and Housing Needs . 35

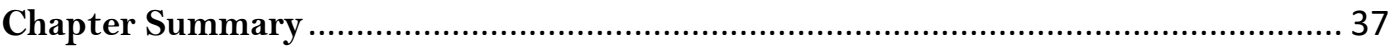

CHAPTER TWO

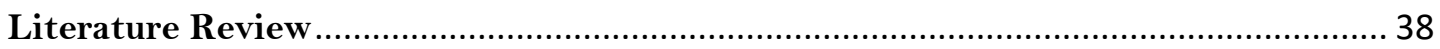

Approach to Reviewing the Literature.......................................................... 38

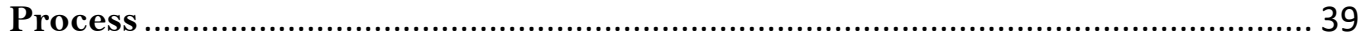

Critical Overview: theoretical foundations of the thesis Social Capital: Bourdieu, Coleman and Putnam ............................................................................... 41

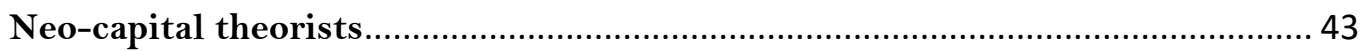

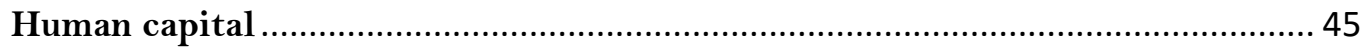

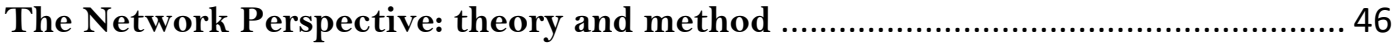

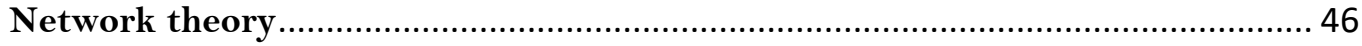

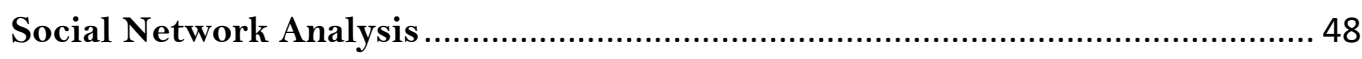

Applied social network analysis studies ................................................ 50

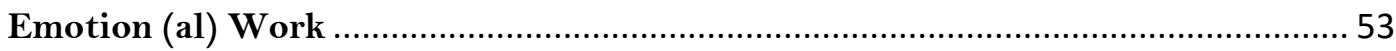

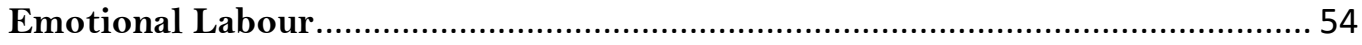

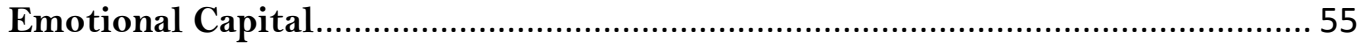

Conclusions from the Literature Review......................................................... 56

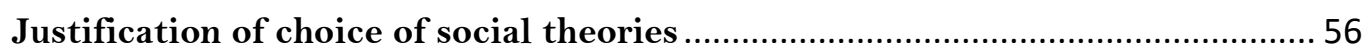

Conceptualising the Caseworker role ........................................................ 60 


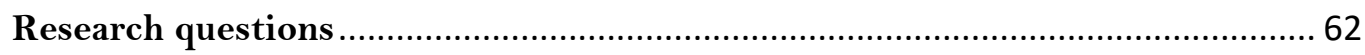

Chapter Summary ...................................................................................... 63

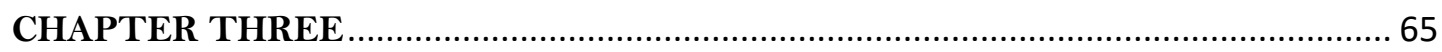

Ontological and Epistemological Foundations of the Thesis ................................. 65

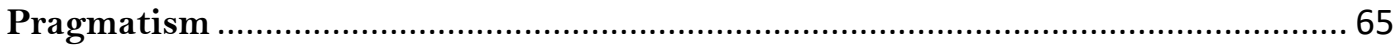

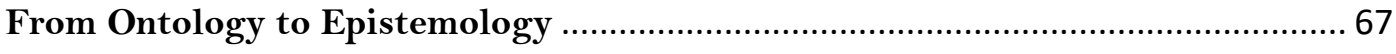

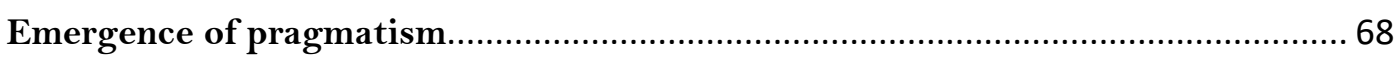

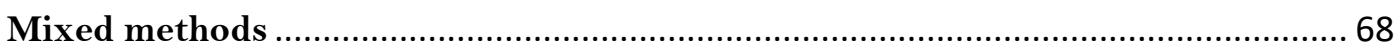

Mapping the reality of the Caseworker network: a social network approach ......... 69

Methodological approaches to data collection ............................................. 70

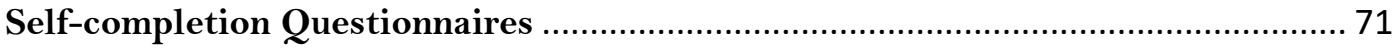

Semi-structured Interviewing: individual face-to-face interviews........................ 72

Semi-structured interviewing: focus groups ................................................... 74

Rationale for choosing pragmatism and a mixed methods design ...................... 75

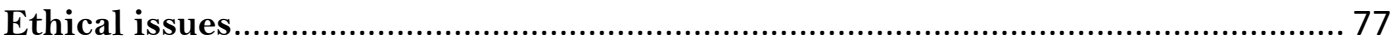

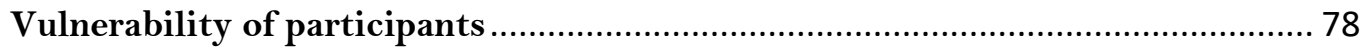

Confidentiality, Anonymity and Informed Consent ......................................... 78

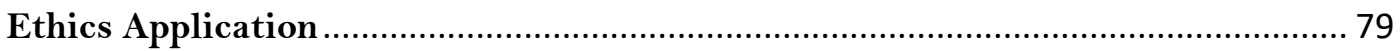

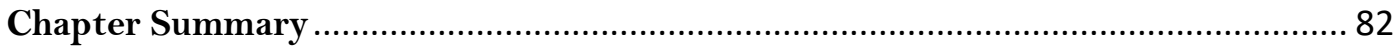

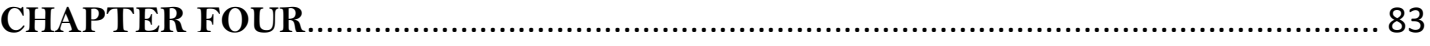

Research Design, Data Collection and Analytical Techniques .............................8 83

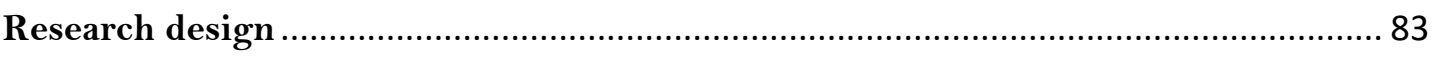

Exploratory sequential mixed methods approach....................................... 83

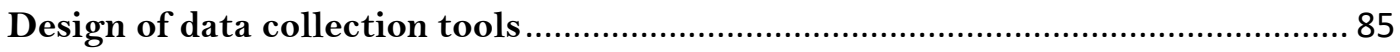

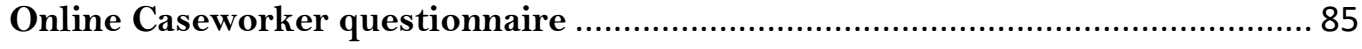

Semi-structured interview schedules for Chief Officers and clients ................. 86

Semi-structured interview schedules for Technical Officers and Caseworker ... 86

Study populations, sampling frames, recruitment and data collection .................. 87

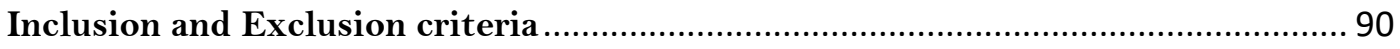

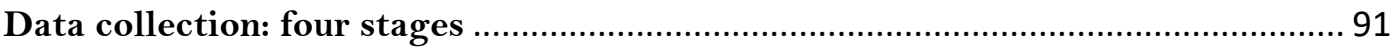

Quantitative data preparation and analysis ............................................. 92

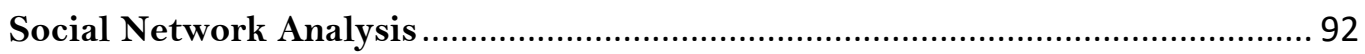

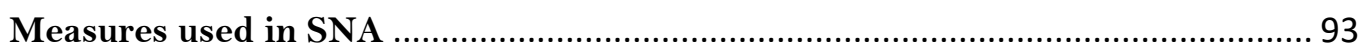

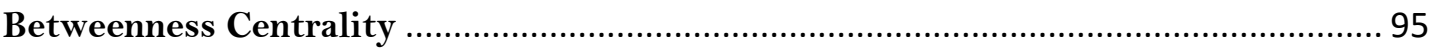

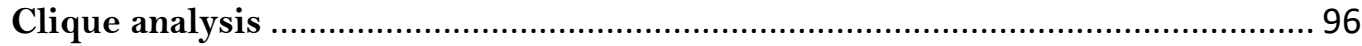

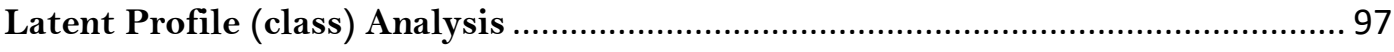

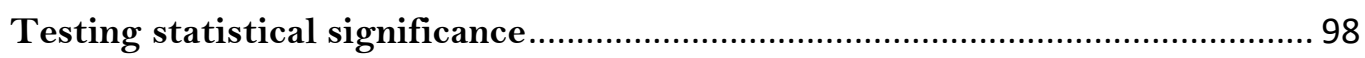

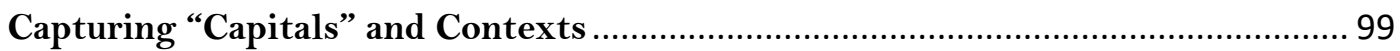

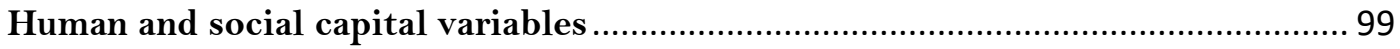

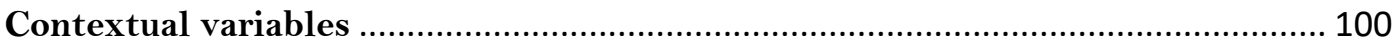

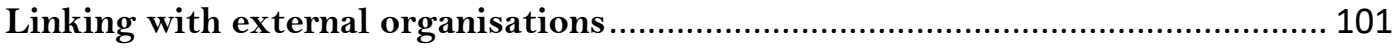


Frequency and breadth of external links.......................................................... 101

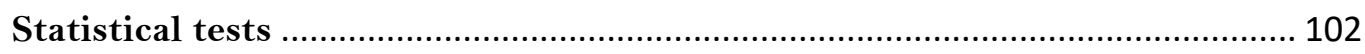

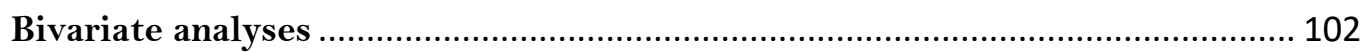

Qualitative data preparation and analyses .............................................................. 103

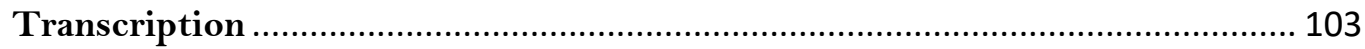

Thematic analysis and coding ............................................................................ 104

How the coding framework was developed...................................................... 104

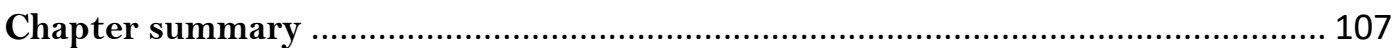

CHAPTER FIVE

Presentation of Findings - Part One: Networking within Care \& Repair................... 108

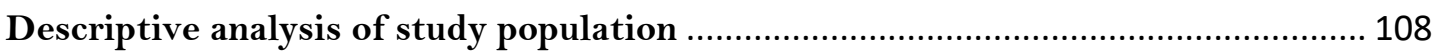

How Caseworkers are connecting with each other ............................................... 110

The numbers of connections Caseworkers make ................................................ 110

Caseworkers as bridges or "connectors" ............................................................ 115

Patterns of interaction within the Caseworker network .................................... 117

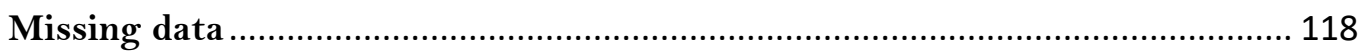

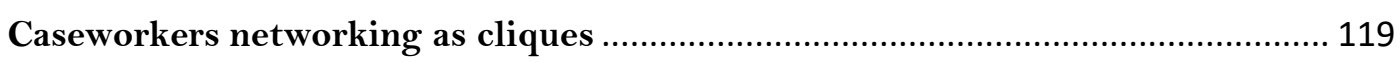

Types of Caseworker connections: patterns of networking................................... 123

Explaining differences in connectivity in the Caseworker network ....................... 125

Factors close to explaining numbers of Caseworker connections ......................... 127

Factors which are associated with patterns of networking ................................ 128

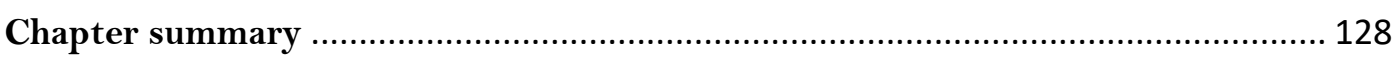

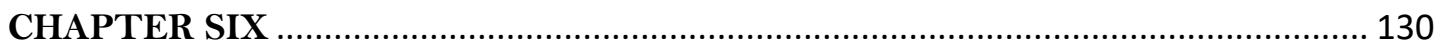

Presentation of Findings- Part Two: How Caseworkers Link with External Services

The importance of inter-organisational working ................................................ 130

The organisations that Caseworkers connect with............................................... 131

How frequently do Caseworkers link with these organisations? .......................... 132

What factors shape the frequency of contact with these organisations? ............. 133

Social and Human Capital factors and frequency of contact ............................... 134

Contextual Variables and Frequency of Contact................................................. 136

The breadth of connections Caseworkers have with external organisations ........ 138

What factors are associated with breadth of connections? ................................... 139

Qualitative insights: Chief Officer perspectives on how the local context influences the Caseworker role............................................................................. 144

Relative deprivation, rurality and links with external organisations................. 145

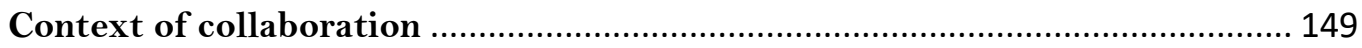

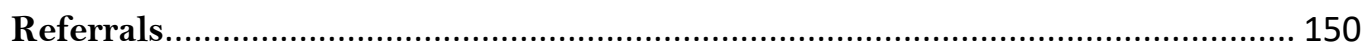

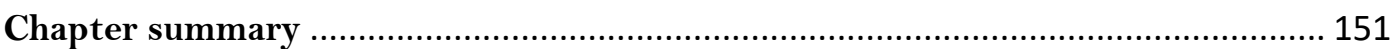

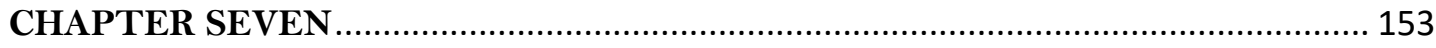

Presentation of Findings - Part Three: The 'Care’ Aspect of Care \& Repair ............ 153 
Section One: Emotional engagement of Caseworkers from Care \& Repair Cymru's

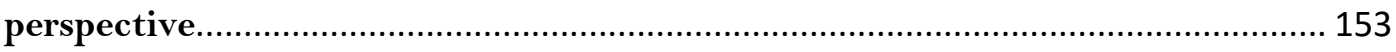

Building up a relationship with a client ........................................................ 154

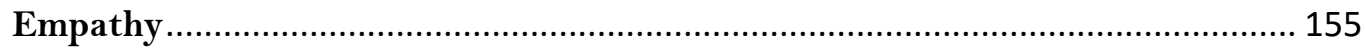

Going the extra mile...Caseworker perspectives.............................................. 157

Section Two: Client experiences of the services .................................................. 159

Trustworthy and professional service ............................................................. 159

Follow-up service: Caseworkers 'being there’ for clients..................................... 161

Emotional engagement of the Caseworkers: client's perspectives .................... 161

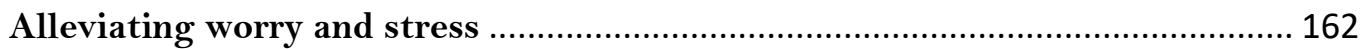

Confidence and reassurance in service delivery or contractors ........................ 163

Emotion work and the Caseworker role ................................................................... 165

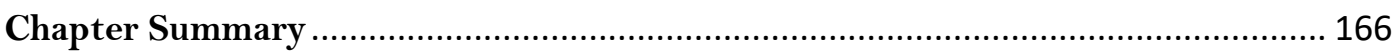

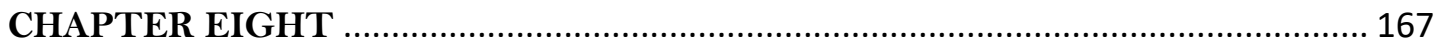

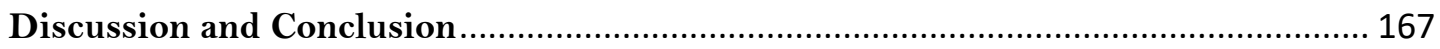

How Caseworkers are connecting with each other ................................................ 167

Explaining these networking connections ....................................................... 168

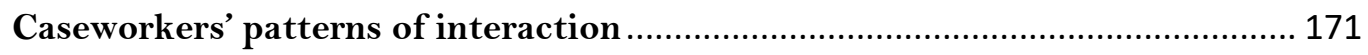

Caseworkers linking across agencies ............................................................ 172

Working collaboratively with organisations beyond Care \& Repair Cymru......... 173

Factors that influence the number of external organisations Caseworkers

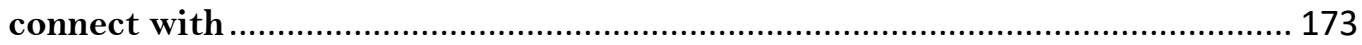

Factors that shape how often Caseworkers connect with external organisations

Experiences of the Caseworker service: 'emotion works'! ..................................... 177

Implications of the research for government and Caseworkers in the current policy

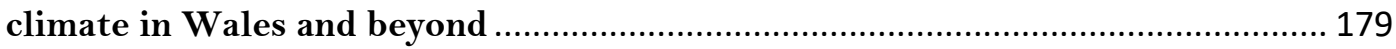

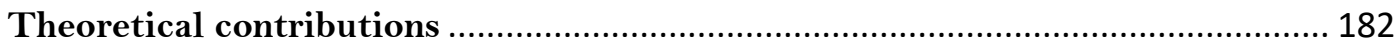



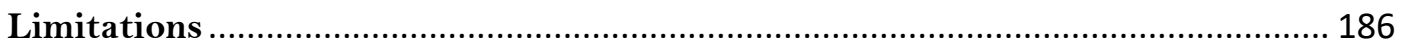

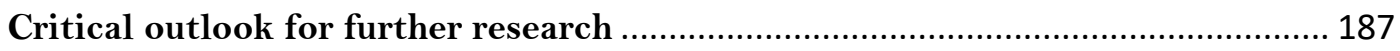

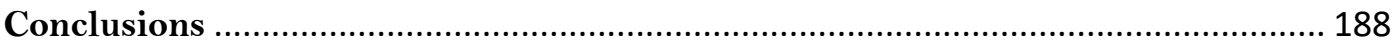

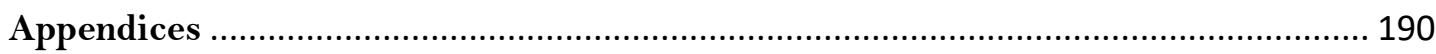

Appendix Item 1- Illustration of the ontological and epistemological

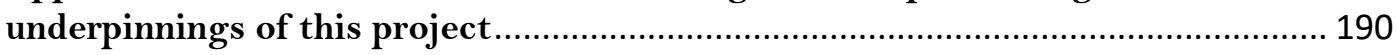

Appendix Item 2: Information Pack for Care \& Repair Cymru Caseworkers........ 191

Appendix Item 3: The Self-completion Caseworker Questionnaire ........................ 197

Appendix Item 4: Chief Officer Interview schedule................................................ 226

Appendix Item 5: Client Interview schedule.......................................................... 228

Appendix Item 6: Technical Officer Interview schedule........................................ 229

Appendix Item 7: Caseworker interview schedule for focus groups....................... 232

Appendix Item 8- Client information pack ……………………………................ 233

Appendix Item 9- Figure showing part of the Microsoft Excel SNA matrix ........ 239 
Appendix Item 10- Technical explanation of the normalisation of SNA measures

Appendix Item 11- Coding of independent variables

Appendix Item 12- Portion of the coding matrix for the qualitative interviews with clients

Appendix Item 13- Frequency distribution of capital and socio-demographic characteristics of Caseworkers

Appendix Item 14- Centrality scores of non-responders to the caseworker questionnaire.

Appendix item 15- Technical explanation of the Latent profile Analysis output 250 


\section{Acknowledgements}

I first wish to thank my primary supervisor Dr Sarah Hillcoat-Nallétamby. I cannot thank you enough for your dedication, patience and encouragement over the years. I certainly would not have overcome the challenges of the $\mathrm{PhD}$ journey or developed my research and presentation skills without you pushing me forward and challenging me intellectually.

I also want to thank the rest of my supervisory team Professor Vanessa Burholt and Dr Helen Daniels for providing their time, expertise and guidance throughout my candidature. Without your involvement this thesis could not have been completed successfully.

Thank you to the staff at Care \& Repair Cymru and its regional agencies for not only being involved in the project but for all their help and logistical support throughout this research project. Without this level commitment the data would have been more difficult to collect, and this study would have been impossible to complete. In particular, I want to thank Neil Williams, Vera Brinkworth and Chris Jones of Care \& Repair Cymru whose unwavering enthusiasm made the completion of this study possible. I would also like to extend my appreciation to all the participants of this study including Care \& Repair Cymru agency Caseworkers, Chief Officers, Technical Officers and Clients who graciously gave up their time to be involved in the study. I want to express my gratitude to Care \& Repair Cymru and the Economic and Social Research Council (ESRC) for awarding me a full studentship to undertake this PhD. This support was crucial, and the undertaking of this research project would have been far more difficult without this.

I would also like to send my appreciation to my family and friends, particularly my Mum, Step-father, Sister and partner's parents for their continuing support and encouragement. Thank you for attempting to help in any way you could. You were all so understanding and supportive and I cannot thank you enough.

I want to thank my partner Dr Matthew Roberts for his sage advice in the later stages of this project and for proof-reading all my chapters. Thank you for being my rock, encouraging me to keep going and being my shoulder to cry on through the ups and downs of this PhD journey.

Thank you to my good friend Dr Lucy Dearn for listening to my PhD and general life woes. You gave me the small boosts (along with a glass of wine) that I needed to keep going and complete my $\mathrm{PhD}$ candidature.

Finally, I would like to acknowledge the support offered by my peers and colleagues within the Centre of Innovative Ageing and the College of Human and Health Sciences at Swansea University. Thank you to the administrative team of Maria Davis, Katie Retallick and Sue Williams for answering my many questions and being 
the friendly faces of the college. I would also like to thank my fellow PhD candidates: Amy Murray, Caitlin Reid and Carole Butler in particular. Thank you all for being friends as well as colleagues.

Thank you everyone for sharing the highs and lows of the PhD journey with me. Without the support from all of you, completing this thesis would not have been possible. 


\section{List of Tables}

Table 4.1-Illustration of creation of themes from word query search

Table 4.2-Illustration of the themes that were identified in the client interviews

Table 5.1-Descriptive statistics for the degree centrality scores for 55 Caseworkers with complete data

Table 5.2-Summary of the individual indegree and outdegree scores of Caseworkers

Table 5.3-Descriptive statistics for 'Betweenness centrality' scores for 55 Caseworkers with complete data

Table 5.4- Summary of the individual betweenness centrality scores of Caseworkers

Table 5.5-Comparison of betweenness centrality and degree centrality scores

Table 5.6-Illustration of the numbers of clique sizes found in the Caseworker network

Table 5.7-Latent profile analysis using MPlus

Table 5.8-Observed characteristics of the most probable class membership

Table 6.1-Freqeuncy (\%) of contact with external organisations ( $n=55)$

Table 6.2- Summary of the breadth of connections Caseworkers make with external organisations

Table 6.3-Summary of key findings: mean scores for association between the length of time in role and breadth of connections with external organisations

Table 6.4-Summary of key findings: mean scores for association between agency location and the breadth of connections with external organisations

Table 6.5-Summary of the key findings: mean scores for association between the issue of 'client is unhappy about changing their home environment and breadth of connection with external organisations 


\section{List of Figures}

Figure 2.1-Illustration of the processes involved in the review of the literature

Figure 2.2-Simple 'Star network' sociogram. Adapted from (Seed, 1990, p.10)

Figure 4.1-Stages of the exploratory mixed methods research design

Figure 4.2- Illustration of indegree and outdegree measures between Caseworkers

Figure 4.3- Illustration of Betweenness Centrality measures for Caseworkers

Figure 5.1-Sociogram of the Wales-wide Caseworker network

Figure 5.2-Illustration of Clique size 5 (1)

Figure 5.3-Illustration of Clique size 5 (2)

Figure 5.4- Illustration of Clique size 5 (3)

Figure 5.5- Illustration of Clique size 5 (5)

Figure 5.6-Illustration of Clique size 6

Figure 8.1-Illustration of the conceptualisation of the Caseworkers as link agents 


\section{List of Abbreviations}

Abbreviation Meaning

\begin{tabular}{l|l}
\hline C\&RC & Care \& Repair Cymru \\
CW & Caseworker \\
TO & Technical Officer \\
CO & Chief Officer \\
SNA & Social Network Analysis \\
LPA & Latent Profile Analysis \\
BG & Blaenau Gwent \\
Bridge & Bridgend \\
C\&V & Cardiff and the Vale \\
Caer & Caerphilly \\
Carm & Carmarthenshire \\
Con\&Den & Conwy and Denbighshire \\
Gwy\&Ang & Gwynedd and Anglesey \\
Mon\&Tor & Monmouthshire and Torfaen \\
MT & Merthyr Tydfil \\
NEW & North East Wales \\
Nport & Newport \\
NPT & Neath Port Talbot \\
Pow & Powys \\
RCT\&MT & Rhondda Cynon Taf \\
Swan & Swansea \\
WW & West Wales \\
SIP & Strategic Insight Programme \\
&
\end{tabular}




\section{INTRODUCTION}

This introductory chapter gives a brief overview of the rationale for the choice of research topic and aims, the broad research objective and the methodological and theoretical approaches which underpin the thesis. These have to some extent, been shaped both by funder requirements, and the broader UK and Welsh policy and practice environments operating at the time, which address the role of housing adaptation services in facilitating ageing in place for older people living in Wales. It ends with a synopsis of the chapters which constitute the rest of this thesis.

\section{Rationale for research}

Ageing in place, or enabling older people to remain living independently 'in their own home and community safely, independently, and comfortably, regardless of age, income or level of intrinsic capacity' (WHO, 2015, p. 36) by receiving personalised services (Phillips, Ajrouch, \& Hillcoat-Nallétamby, 2010), is a topic that has been given widespread attention within UK policy, service development and academia. In the UK context, independent living (see Chapter One for definition) has informed and underpinned policies directed towards older people and housing (Means, 2007; Sixsmith \& Sixsmith, 2008). However, policy makers have recently begun to question the idea that ageing in place at home is the best and preferred option for all older people (Hillcoat-Nalletamby \& Ogg, 2014). Instead, there has been a much more recent emphasis in policy on ensuring that older people are provided with the choice to either age in place or move on to more suitable accommodation (Communities and Local Government Committee, 2018; Expert Group on Housing an Ageing Population in Wales, 2017).

To date, there has been a wealth of research into understanding the notion of ageing in place and what influences and shapes it as a residential option. For instance, the theory of attachment to place examines how an individual's emotional attachment to their environment influences their choice of living arrangement as they grow older (Burholt, 2006; Hillcoat-Nalletamby \& Ogg, 2014; Wiles, Leibing, Guberman, Reeve, $\&$ Allen, 2012). Other research has examined individual experiences and challenges associated with ageing in place (Davey, 2006; Fausset, Kelly, Rogers, \& Fisk, 2011 ; Stones \& Gullifer, 2016); and whether offering different housing options and chances for residential mobility should be considered in policy and discussions about ageing well and living arrangements in later life (Boldy, Grenade, Lewin, Karol, \& Burton, 2011; Expert Group on Housing an Ageing Population in Wales, 2017; HillcoatNalletamby \& Ogg, 2014). Additionally, there has been research examining the efficacy of different services or approaches to facilitating ageing in place, for example 
adaptions to buildings to accommodate different levels of health and mobility needs (Fielo \& Warren, 2001; Powell et al., 2017; Procter et al., 2014).

However, much less research and attention has been directed towards examining the individuals who deliver these services at the grass roots level by actively working to ensure that older people can remain living in the community for as long as possible. This is most notably true in the Welsh context. Given the limited research in this area, the thesis will help address this knowledge gap by exploring the role that Care \& Repair Cymru Caseworkers potentially play as link agents ${ }^{1}$ - individuals who connect and work in a professional capacity with fellow Caseworkers, and others across health, housing and social care sectors to facilitate service delivery so that older people can age in place independently.

Care \& Repair Cymru (C\&RC) is a social enterprise and charity in Wales dedicated to improving homes and facilitating independent living for older people (Care \& Repair Cymru, 2016b, 2017a; Davison, 2012). As an organisation, they are in receipt of core funding ${ }^{2}$ from Welsh Government and operate 13 home adaptation agencies across Wales, which together employ a total of 75 Caseworkers. As an organisation, their work in facilitating the delivery of home adaptation and other services to older people living at home means that they play a pivotal role in facilitating ageing in place and independent living.

This research is timely when considering the UK context of general welfare retrenchment, issues of social care costs for an ageing population, and government priorities which espouse closer cross-sector and partnership working to stream line services and increase efficiency (see Chapter One). It is doubtless now an understatement to say that many organisations or services which benefit from public funding are faced with the paradoxical challenge of meeting the demands of an ageing population but with fewer resources at their disposal (Griffiths \& Kippin, 2017; Moriarty, Manthorpe, \& Cornes, 2015). Therefore, there is increased pressure to ensure efficiency and service development and delivery. To cope with this pressure, a common management strategy involves the formation of partnerships with other organisations based on shared goals (Leslie \& Canwell, 2010; Lowndes \& Squires, 2012). Indeed, in the context of austerity, there has been a growth in initiatives to

\footnotetext{
${ }^{1}$ A full explanation of what the term link agent means and the theoretical literature from which this term is derived will be examined in Chapter Two- Literature review.

${ }^{2}$ In terms of funding, Care \& Repair Cymru are a charity but do receive core funding from the Welsh Government which is supplemented by their own in-house social enterprise schemes and charitable donations.
} 
foster partnerships between health, social care and third sector organisations (Glasby \& Dickinson, 2014). Partnership working and networking is therefore common practice amongst many organisations and has been shown to offer strategic advantages (McQuaid, 2000), including a more efficient basis for sharing existing resources (Edwards, 2010), or as a means of attracting additional resources (McQuaid, 2000) such as funding. More critically speaking however, there are several recognised challenges in promoting partnership working on the ground including; negotiating resource sharing; reconciling different organisational aims and objectives and regulatory differences (Provan \& Milward, 2001).

Partnership and collaborative working have also become integral to policy discourse (Mur-Veeman, Hardy, Steenbergen, \& Wistow, 2003; Terry, 2017; Welsh Government, 2011,2014d), and in some instances this has become the norm (Glasby \& Dickinson, 2014, p. xiii). This is particularly evident in the Welsh context, where there the emphasis is not so much on facilitating collaborative practices, but rather justifying instances where this is not occurring (Glasby \& Dickinson, 2014; Welsh Assembly Government, 2011). The recent implementation of the Wellbeing and Future Generations (Wales) Act 2015 has been particularly significant, because stronger collaboration and new ways of working between different sectors and organisations is viewed as a mechanism to achieve the long term goals of ensuring Welsh citizens live in communities that are equal, healthy, vibrant and prosperous (Welsh Government, 2015b). More specifically in relation to this thesis, a recent report by the Auditor General for Wales (2018) examines the value and efficacy of housing adaptation services. It recommends increased partnership working between organisations as a mechanism for addressing the needs of older people effectively and efficiently. This is a timely policy statement which suggests that despite the emphasis on promoting collaborative working, this has yet to be realised in practice.

As with other UK devolved nations, the context in which C\&RC agency Caseworkers operate is not immune to the impact of austerity and reductions in public expenditure in the broader demographic context of population ageing. Throughout the duration of this thesis, this national-level organisation has undergone quite significant restructuring, with a reduction of its agencies from 22 to 13 by 2016. This notwithstanding, it has managed to maintain an overall Caseworker workforce of between 75 and 78 individuals (Care \& Repair Cymru, 2016a, 2017a). A recent internal evaluation report (Care \& Repair Cymru, 2016b, p. 12) suggests that their work has cost savings effects, with estimates of savings from health and social care budgets of $£ 7.50$ for every $£ 1.00$ the Welsh Government invests in its Rapid 
Response Adaptations Programme (a housing adaptation service for minor works e.g. installation of handrails, which aids older people to safely return to their homes following hospital discharge and reduces the likelihood of admission to residential care).This points to the financial viability of the services delivered by Caseworkers, which save on already restricted health and social care budgets.

In theory, C\&RC is well placed to respond to the broader objectives of cross-sector and partnership working to facilitate more effective service delivery, because their remit involves working across and with health, social care and housing providers (Care \& Repair Cymru, 2016a, 2017a; Davison, 2012). Caseworkers are, on the face of it, and by the nature of their work with older clients, key actors in this endeavour. However, when this research was commissioned, there was no robust empirical evidence which either documented or explained the role C\&RC agency Caseworkers may play in achieving cross-sector and partnership working.

Anecdotal and isolated service developments across Wales where Caseworkers play a central role does, however, point to this potential. For example, a 3-year project, the 'Managing Better Sensory Service', involved the appointment of 13 new specialist Caseworkers. These individuals are tasked with working with their local health and social care services to identify older people who may be vulnerable and require help to ensure that their home environments are safe and healthy, before a crisis point is reached. These actions indicate the potential for 'Managing Better Caseworkers' to effectively fulfil a link agent role, by facilitating cross-partnerships working (Care \& Repair Cymru, 2016a).

Other service initiatives such as those of a pilot 'Moving On' service in North Wales (Hillcoat-Nalletamby \& Sardani, 2015), funded by Wales' Intermediate Care Fund, relied on the work of a single Caseworker (as part of a small management team) to facilitate the decisions and practical steps involved in older clients making the move from their home to an extra care facility. This individual worked in a link agent capacity by connecting with different services including energy companies, solicitors and removal services, to make moving in later life a less stressful experience.

A 'Hospital to Home' service in Bridgend has relied on the networking skills of two of Caseworkers to ensure that hospital and care staff work in tandem with older clients to ensure a safe and timely return to their home. A similar service Healthy Homes for Discharge in North East Wales has been established by agencies in Newport, Flintshire and Wrexham (Care \& Repair North East Wales, 2018). A de-cluttering service, the 
New Attic Project is effectively delivered on the ground in Cardiff, Newport and the Vale of Glamorgan by Caseworkers who, in collaboration with volunteers, assist older clients with the complex and daunting task of de-cluttering their homes, to facilitate home improvements, or with a view to starting a discussion about relocation. Affordable warmth services are delivered in North East Wales (Flintshire and Wrexham) and Carmarthenshire which are funded by the Big Lottery and aim to advise and assist on issues pertaining to energy efficiency and reducing costs. As part of this service Caseworkers will liaise with energy companies and contractors to tackle these issues.

In sum, service initiatives in which Caseworkers play a central role are continually evolving. However, there is no empirical evidence for Wales to demonstrate how this potential for partnership working is really developing on the ground, in relation to a key national-level housing adaptations service provider. Furthermore, the Caseworkers potentially bring a whole host of skills, knowledge and experience to their diverse roles as these service initiatives illustrate.

As its overall objective, the thesis will therefore explore Care \& Repair Cymru Caseworkers in terms of their potential to be link agents who foster cross-collaborative working, by examining the professional network of relationships and links they develop within and beyond their own organisation, and the skills and expertise they bring to bear on this. To achieve this, the thesis will:

1. Use theory and empirical evidence to propose a conceptual framework of the Caseworker as a link agent.

2. Visually map the networking activities that Caseworkers across Wales have developed with each other by using Social Network Analysis techniques; and identify patterns and explanations for these networking activities;

3. Establish and explain the types of professional partnerships that Caseworkers have developed with other organisations;

4. Explore client perceptions of Caseworker interventions.

To this end, the thesis adopts a pragmatic mixed method approach and has involved data collection from all Care \& Repair Cymru agencies operating across Wales during the completion of the research. This includes information about Caseworker activities within their respective agency areas at the community level, mainly using an online survey. Data derived from this questionnaire are analysed using social network 
approaches and statistical analyses including latent profile analysis and other tests including analysis of variance. The results of these analyses are interpreted against a backdrop of contextual information provided by agency managers (Chief Officers) during face-to-face, in-depth interviews and focus groups with Technical Officers and Caseworkers. A small number of in-depth face-to-face interviews with Care \& Repair Cymru agency clients provide insights into service-user perspectives about the organisation and the Caseworker service and are subject to thematic analysis.

\section{Mapping and explaining Caseworkers as link agents}

Subsequent chapters outline in more detail the reasons why a pragmatic mixedmethods approach has been adopted for data collection and analysis. This includes the reasons for selecting certain theoretical approaches to help interpret and make sense of empirical findings.

Mapping the network with Social Network Analysis: in terms of mapping the Caseworkers' professional networking activities with each other, the thesis draws on social network analysis and network theory to help visually map and create measures which can be used to explain the types of links they develop as part of their broader professional network (the Caseworker network). Social and human capital theory are used to interpret these findings in terms of the relationships, skills and education which Caseworkers draw on as part of their collaborative working.

Network theory reflects the core assumption that human beings are social animals who are connected, and these connections form the basis of social structures and organisations (Borgatti \& Li, 2009). This differs from social network analysis, which can be conceptualised as a method of capturing and investigating these social structures or relationships (Tichy, Tushman, \& Fombrun, 1979) as a means of offering unique insights into how individuals connect with each other. For example, this analysis can help identify which actors can be considered important for a particular network, whether they act as bridges connecting disparate factions of the network, or are perhaps key brokers of information or highly connected to a number of people, and so are integral to the structure of the network (Burt, 1997; Nicaise et al., 2012).

The usefulness of social network analysis for the purposes of examining how individuals are linking has been noted in previous works (Cross, Borgatti, \& Parker, 2002; Marin \& Wellman, 2011; Tichy et al., 1979). 
'In order to fully understand the behaviour and policies of organisations involved in economic development and regenerations it is necessary to consider the nature of their relationships with networks of and partnerships between other actors, including the flows of resources, power and information within these networks' (McQuaid, 2000, pp. 1-2).

Although McQuaid (2000) was referring to public-private urban regeneration and economic development partnership projects, the points stated can be applied widely to any organisation. Chan and Liebowitz (2005), state that mapping how individuals interact "provides a basic tool for managers and employees to retrieve the necessary knowledge and to analyse the relationships between knowledge sources' (p. 21). Although the authors were discussing social network analysis in the context of creating knowledge maps in the corporate and business sector, this statement partially informs the rationale underpinning this thesis. This approach to understanding a given workforce allows a researcher to illustrate what the network looks like in addition to developing possible explanations of why or how it works.

Therefore, the findings from social network analysis studies have several potential implications for service development, which includes enabling managers to be aware of the kinds of expertise and knowledge contained within their workforce and increasing their awareness of the internal networking that may or may not be taking place and why.

In relation to this thesis, while social network analysis can help visually map the links that Caseworkers are making, the social theories of social and human capital enable interpretation and explanation of the links observed. Ennis and West (2010) argue that social capital theory and social network analysis are interrelated because social networks are the structure in which social capital exists. This thesis considers social capital to be an individual level asset representing the capital of relationships and the resources (prestige, knowledge, skills and contacts) embedded within them (Bourdieu, 1973; Coleman, 1988; Putnam, Leonardi, \& Nanetti, 1994). These relationships and hence social capital are essential to sharing information, resources, and facilitating social change (Ennis \& West, 2010) and are important to grasp in a policy context of austerity and promotion of collaborative working. A critical review of different social capital theories and the function they serve will be explored in greater depth in Chapter Two.

Human capital refers to the skills, education and training that individuals invest and develop over time, which can be accessed to obtain some form of advantage (Becker, 1962, 1994; Coleman, 1988; Schultz, 1961). These social theories will be used as a 
theoretical lens to explore whether there are associations between the dynamic of the network, the structural position that individuals occupy in this network and the competencies that they bring into the role.

Establishing the links with other external organisations: social and human capital theories will also provide important interpretive perspectives in understanding the factors which shape the professional relationships that Caseworkers build with organisations and services external to Care \& Repair Cymru agencies.

The emotional component of links with clients: the thesis also draws on emotion work theory as neither network theory, social network analysis nor social and human capital theories are designed to help capture the more subjective aspects of relational exchanges which may occur between client and Caseworkers. Emotion work can be understood as 'behaviours performed to improve emotional wellbeing in others and to create cooperative and positive social relationships' (Strazdins, 2000, p. 41). This theory provides an interpretive framework, which will enable an exploration of the interpersonal support component of the Caseworker role from the client's perspectives.

To summarise, ageing in place has been a prevalent topic within UK policy, service provision and academia with research around this subject typically focused on the services developed to achieve this. This notwithstanding, there has been little research which focuses specifically on understanding how those delivering services on the ground actually operate to make independent living at home a reality in the community. The thesis aims to address this gap for the Welsh context in particular, by examining a specialised workforce of Care \& Repair Cymru agency Caseworkers, who potentially hold a unique place and role as link agents in promoting ageing in place through partnership working, notably through their work with health, housing and social care services and organisations. An exploration of Caseworkers and their professional internal network is well-timed considering the emphasis placed on increasing collaborative and cross-sector working in response to wider societal issues e.g. ageing population, welfare retrenchment and increasing social care costs.

\section{Organisation of Chapters}

The thesis is comprised of eight chapters. Chapter One discusses the policy, social, cultural and demographic context of Wales. Its overall aim is to set the contextual scene in which the research is grounded. It will outline the population of older people in Wales, the policy responses put in place to address the needs of this growing population and notions of ageing in place and independent living which are central 
to the development of these policies. The challenges of enacting these policies and achieving independent living through ageing in place will also be discussed. Finally, this chapter will provide a background to Care \& Repair Cymru including their aims and objectives, in addition to outlining how the Caseworkers fit into the organisation and the potential role they play in delivering services that facilitate independent living and ageing in place.

Chapter Two provides a review of the literature and studies that have informed the research design and questions that this thesis seeks to answer. First, the narrative approach to identifying and reviewing previous literature will be explained. This chapter then discusses social capital theories from both traditional (Bourdieu, 1973; Bourdieu \& Wacquant, 1992; Coleman, 1988, 1990; Putnam, 2000; Putnam et al., 1994), and neo-capital (Lin, 1999, 2001) perspectives including their shortcomings . Then human capital theory (Becker, 1962, 1994; Beine, Docquier, \& Rapoport, 2008; Coleman, 1988), network theory (Borgatti \& Halgin, 201 1; Borgatti \& Li, 2009; Ennis \& West, 2010), social network analysis studies (Coleman, Katz, \& Menzel, 1957, 1966; Nicaise et al., 2012; Woodcraft, 2011), emotion work (Erickson, 1993; Strazdins, 2000), emotional labour (Bolton, 2005; Hochschild, 2003; Lopez, 2006) and emotional capital (Feeney \& Lemay Jr, 2012; Gendron, 2004; Williams, 1998) theories will be explored. Conclusions derived from a review of this literature will then be discussed which will detail how these theories and approaches have shaped the research questions, research design and the interpretation of findings.

Chapter Three reviews the theoretical and conceptual frameworks which have shaped the research design and approach. This chapter begins by exploring pragmatism as a research paradigm which incorporates both objectivism and constructionism into a single research design and its relevance and suitability to this thesis. Pragmatism is associated with a mixed methods approach because it views both the world and knowledge to be both objective and constructed; therefore, research should aim to capture this by using methods which best address these dimensions. It then outlines why a mixed methods approach was adopted over a mono-methods approach. This is followed by a discussion of the advantages and disadvantages of the data collection tools in addition to outlining the rationale underpinning the use of these methods in this thesis. Finally, the chapter outlines the ethical issues that had to be considered prior to conducting the fieldwork.

Chapter Four examines the research design and processes undertaken to answer the research questions. The first section explores the overall research plan, including the 
tools developed to gather the data. The second part of this chapter outlines the four study populations, recruitment frameworks and strategies used to recruit participants. This chapter then proceeds to outline how quantitative data was prepared for different kinds of analyses. This section also describes the different kinds of quantitative analyses used to answer the research questions including social network analysis and statistical tests. Finally, this chapter discusses how the qualitative data was prepared for analysis including data transcription and thematic analysis.

Chapter Five presents the key findings that emerged from the social network analysis and the examination of the factors associated with how Caseworkers network with each other. This chapter will illustrate that Caseworkers were networking in different ways and that their patterns of networking can be typified high connectors or low connectors. Similarly, this chapter shows that statistical tests indicate that capital factors (the length of time a Caseworker has been in post and having previous work experience in the business sectors) were associated with the different ways in which Caseworkers network.

Chapter Six presents the results from the analyses that examine the types of connections that Caseworkers make with external organisations and statistical tests of association. Specifically, the results indicated that both individual (capital) and contextual factors associated with the different ways in which Caseworkers link with external services.

Chapter Seven explores some of the findings pertaining to the caring aspects of the Caseworker role. This chapter outlines the findings which indicate how emotion work is realised through the Caseworkers' job role from the perspective of Care \& Repair Cymru staff and clients.

Chapter Eight will consider and discuss the main findings of the project within the theoretical framework of social and human capital theories, network theory and emotion work theory. This chapter will also outline the limitations of this study and considers opportunities for further research. Finally, the main conclusions are stated. 


\section{CHAPTER ONE}

\section{The Policy, Social, Cultural and Demographic Context in Wales}

\section{Policy response to an ageing population in Wales}

This chapter explores the Welsh context in which the thesis is grounded and begins by describing the ageing population of Wales. This includes an examination of the policy response made by the Welsh Government to address the needs of an ageing population. Definitions will be given for independent living and ageing in place as these concepts underpin Welsh policies around older people and have shaped service delivery and practical responses to meeting the needs of an ageing population. A critical appraisal of the challenges associated with trying to achieve ageing in place and independent living is then provided, including health related changes and social, cultural and demographic variations which have resulted in changing family structures, roles and expectations. The quality of the housing stock in Wales is then discussed because this can impede the ability of older people to age in place. The chapter ends with an outline of Care \& Repair Cymru and the work they undertake to facilitate independent living through ageing in place and addressing the needs of the population of older people in Wales.

Like most other devolved nations of the UK, Wales is experiencing an ageing population (Baxter \& Boyce, 2011; Collerton et al., 2007). According to the last census in 2011, approximately 18 per cent of the population in Wales are aged 65 years and above. This equates to a total of approximately 563, 000 individuals, and of this number there were 25,000 Welsh residents who are aged 90 years and above in 2011, compared with 19,000 in 2001 and 700 in 1911 (ONS, 2012). Furthermore, the number of individuals aged 65 years and above is projected to increase by 36.6 per cent between 2016 and 2041 (Welsh Government, 2017b), indicating that the population of older people itself is also ageing. This is reflected in broader UK population trends. In 2016 there was an estimated 14,910 individuals aged 100 years and over, and the proportion of the population aged 90 and above had grown more rapidly than younger cohorts (Office for National Statistics, 2017b; Tomassini, 2005). This increase in longevity and the increasing number of the oldest older people can be attributed to a number of factors, including a decline of mortality and birth rates (Hamza \& Gilroy, 2011; Moody, 2010). 
For the purposes of this research, individuals aged 65 years and over are considered, older people. This definition was influenced on the one hand by the classification of older people by the United Nations which defines older people as individuals aged 60 years and above (WHO, 2002). On the other hand, previous academic literature and UK policy documents tend to equate their definition of old age with individuals aged 65 years or over, mainly because it corresponds to a typical retirement age for men in the UK (Coleman \& Bond, 1990; Roebuck, 1979). However, Wales appears to be an exception, where the definition of 'old' adopted by the Welsh Government refers to individuals who are 50+ years (Carter-Davies \& Hillcoat-Nallétamby, 2015).

\section{Defining Ageing in Place and Independent Living}

The latest census data indicates that the majority of older people in England and Wales are ageing in place by living in the community rather than institutional contexts such as residential care or nursing settings ${ }^{3}$ (ONS, 2012, 2014). The concept of ageing in place has no one set definition, but broadly speaking refers to 'growing old without having to move home' (Phillips et al., 2010, p. 17), and is often aligned with the notion of independent living (Ahlqvist, Nyfors, \& Suhonen, 2016). However, there is no clear or universal definition of this concept, rather, studies including those conducted by Davey (2006); Sixsmith and Sixsmith (2008); and Wiles et al. (2012) appear to make associations between the notion of independence, living arrangements and ageing in place. Independence is a multifaceted and highly relative concept encompassing aspects of autonomy, self-identify and interaction with the physical and social environment (Hillcoat-Nallétamby, 20 14; Rabiee, 2013), although broadly speaking this can be understood as,

'a sense or state of physical, psychological and spiritual autonomy, self-identity, selfrespect, control and a degree of functional capacity' (Phillips et al., 2010, p. 131).

Leeson, Harper, and Levin (2004) conducted a review into independent living in later life, which determined that several individual level factors shape, promote or constrain experiences of independence including: living arrangements; integration into family networks; having a sense of control and empowerment; financial security; and health and social care issues. This thesis understands independent living to be a sense of having autonomy, control, choice, freedom, economic security and functional capacity regarding living arrangements and every day aspects of social life (Ahlqvist et al., 2016; Phillips et al., 2010; Rabiee, 2013; Seidel et al., 2010), which can enable individuals to age in place at home or in the community. Achieving independent living in old age is intimately linked with the ease with which individuals feel they

${ }^{3}$ For England and Wales, $3.2 \%$ of all those aged $65+$ lived in residential care homes. 
can interact and control their living environment (Heywood, Oldman, \& Means, 2002). Therefore, independence has become a central concept underpinning strategies and initiatives working towards ageing in place. Additionally, promoting independence has become more prevalent as care provision for older people have moved from institutional to community settings (Phillips et al., 2010) in England and Wales (Secker, Hill, Villeneau, \& Parkman, 2003).

\section{Policy responses}

The concepts of 'ageing in place' and 'independent living' have become central to policies in Wales and have heavily influenced the development of services and strategies, which address the issues associated with an ageing population (Baxter \& Boyce, 2011; Dawson, Bowes, Kelly, Velzke, \& Ward, 2015). In terms of policy they have been reflected through the work of the Older Person's Commissioner, first appointed in 2008 to coincide with the implementation of the second phase of the Welsh Government's Older People's strategy (2008-2013). The Welsh Declaration of Rights for Older People reflects an understanding of living independently and having a choice over living arrangements to be core components of the rights for older individuals. Whilst the Declaration has no statutory value, it does signal a commitment of the Welsh Government and the Older Person's Commissioner to take the safety and wellbeing of older people seriously (Welsh Government, 2014a).

The focus of the Strategy for Older People in Wales, currently in its third phase which commenced in 2013 and which will last until 2023, is to ensure,

'...that all older people in Wales have the social, environmental and financial resources they need to deal with the opportunities and challenges they face... in ways that go beyond the traditional health and social care agenda'. (Welsh Government, 2013, pp. ii-iii).

In practical terms, these resources entail access to information and social support; having an income either through employment, pension or other means; financial inclusion; having adequate housing that is insulated, safe, secure and adapted to meeting the needs of older people; having access to transport; and being able to live in, and be involved in their local community (Welsh Government, 2014c). This suggests that once an older person has adequate housing and resources in place, then they should be able to age in place and live independently in their local community. To achieve this, the Welsh Government has funded several initiatives to facilitate independent living at home for older people. This is illustrated in a recent report by the Auditor General for Wales (2018) which provides a review of the delivery of housing adaptation services across Wales. It found that approximately $£ 60$ million is spent each year on housing adaptations which are provided by around 70 
organisations (including Care \& Repair Cymru agencies) to over 32,000 individuals across Wales. However, it also notes that the current system for funding is inefficient and highly complex. Because of this, individuals with similar needs can receive different standards or service delivery based on where they live. The report also found that policies surrounding housing adaptation services reflected a focus on the property and not the individuals living within them. It concludes that many service delivery organisations work in isolation from each other, whereas a collaborative approach would enable them to better address individual health and housing needs. Overall, the report shows that housing adaptations constitute a major part of the policy agenda of the Welsh Government, but that the system underpinning service delivery is inefficient and flawed, resulting in inconsistent standards and experiences of services (Auditor General for Wales, 2018).

Other practical initiatives to facilitate independent living at home for older people in Wales include the 'ENABLE-Support for Independent Living' initiative which was launched in 2016 and received $\mathfrak{\&} 4$ million of funding to restructure and regroup the existing system of home adaptation service delivery, such as the Disabled Facilities Grants to make it simpler and more efficient. This initiative recognises the value of home adaptations as a preventative measure for costly hospital admissions and is seen as a key way to promote independence (Welsh Government, 2017a, 2018). Additionally, the Wellbeing and Future Generations Act (Wales) 2015, promotes preventative approaches and collaborative working between organisations to ensure that service delivery and the needs of older people are met (Welsh Government, 2015a).

Since 2003, Welsh Government has also funded the 'Supporting People Programme', which supports vulnerable people with housing related problems to live as independently as possible by taking a preventative approach to service delivery. The programme is also supposed to facilitate collaborative working between organisations to plan and fund services which complement those provided by health and social care organisations (National Assembly for Wales Public Accounts Committee, 2018; Welsh Government, 2017c). The programme is however aimed primarily at reducing levels of homelessness in Wales, so it is not solely focused on the housing needs of older people, e.g. the need for adaptations.

\section{Ageing in place and independent living: the challenges}

Despite the policy and service emphasis on facilitating and achieving ageing in place and independent living for older people, in practise it is becoming increasingly 
challenging for several reasons. First, the impact of the 2008 recession and ensuing reductions in public spending, which the Institute for Fiscal Studies (2017) estimate to have corresponded to $3 \%$ in real terms between 2010-2015, have had an impact in Wales. As most Welsh Government public sector funding is provided by the UK Treasury in the form of a block grant, measures of fiscal consolidation have resulted in reductions to funding, with the consequence of cuts to public spending in social welfare, health and social care and the third or charitable sectors in Wales (Deaner \& Phillips, 2013). This has meant that while the demand for social and support services has increased, the financial resources made available to support these services has been reduced or frozen (Luchinskaya, Ogle, \& Trickey, 2017).

Other factors which challenge the feasibility of promoting or sustaining ageing in place for older people include: health changes (including compression of morbidity, co-morbidity and mobility issues); changes in family structures linked to declining fertility rates (Crimmins \& Beltrán-Sánchez, 2011; Fries, 2005; Office for National Statistics, 2015; WHO, 2015); and changes in gender norms and expectations about caring and paid employment.

\section{Changes to Health}

Health changes potentially reduce the likelihood of older people living independently in their own homes. For instance, the compression of morbidity posits that while most people live the vast proportion of their lives in good health, it is typical for many to experience life-limiting illnesses in a very intense and brief period shortly before death (Crimmins \& Beltrán-Sánchez, 2011; Fries, 2002, 2005). Compression of morbidity is further complicated by the instances of co-morbidity or the presence of additional diseases or conditions alongside a primary diagnosis (Department of Health, 2014; Valderas, Starfield, Sibbald, Salisbury, \& Roland, 2009). Levels of mobility, an individual's capacity to be able to move themselves from one space to another both safely and independently (Rantakokko, Mänty, \& Rantanen, 2013), and mobility impairments or restrictions have been recognised as a precursor to disability (Manini, 2013). Loss of independent mobility is also associated with higher rates of morbidity, poorer quality of life and an increased risk of social isolation (Manini, 2013; Quadri, Tettamanti, Bernasconi, Trento, \& Loew, 2005).

Although compression of morbidity, co-morbidity and declines in mobility are distinct issues, quite often they overlap and become interconnected. As people age, there is a certain expectation that one's physical, cognitive and perceptual abilities (for instance a loss in vision and hearing, and a decline in physical strength) will 
change (Fausset et al., 2011; Hébert, 1997; Small, Dixon, \& McArdle, 2011). However, it is impossible to predict exactly when these changes will occur and what the ramifications are. For instance, a loss in visual acuity could mean that an individual's depth of perception is diminished, making them more prone to falls (Ivers, Cumming, \& Mitchell, 2002; Klein, Moss, Klein, Lee, \& Cruickshanks, 2003; Ray, Horvat, Croce, Mason, \& Wolf, 2008).

These age-related changes increase the possibility that some individuals will undergo periods of time when they are severely ill and may have to deal with multiple illnesses at one time. Consequently, Fausset et al. (2011) suggest that 'age-related changes can pose challenges for an older adult to meet the demands of the environment' (p. 2) making independent living and ageing in place increasingly difficult to achieve. Social gerontologists have already recognised this issue through theoretical and empirical work by scholars such as Lawton (1982) and others' around environmental gerontology. For instance, in their study Sixsmith and Sixsmith (2008) refer to the designs of homes as barriers for older people to age in place, especially as functionality and health decreases.

Similarly, research by Hwang, Cummings, Sixsmith, and Sixsmith (2011) and Johansson, Josephsson, and Lilja (2009) and Peace, Holland, and Kellaher (2011) analysed the relationship between ageing in place and home modifications, and found that person-environment fit (the extent to which an individual's characteristics matches their environment) needed to be considered into service delivery to promote independent living. Having older people remain in their own homes and communities for as long as possible is the preferred living option for health providers and policy makers because this purportedly avoids or at least reduces using costly institutional care (Oswald, Jopp, Rott, \& Wahl, 2011; Tinker et al., 1999). This assumption has however been challenged because it does not consider the quality of the housing in which older people reside. Living in poor quality housing in later life carries a risk of falls (Rubenstein, 2006), poor mental and physical health (Evans, 2003; HowdenChapman, Signal, \& Crane, 1999; Tinker, 1997), and isolation (Sixsmith \& Sixsmith, 2008), which can be costly for health and social care services .

Despite many older people being in good health (Office for National Statistics, 2013; Welsh Government, 2014a), as the population of 'very old' older people (aged 85 years and over) increases, health and social care services, particularly those implemented at the community level, are faced with the challenge of caring for individuals with complex needs (Moriarty et al., 2015; Welsh Government, 2014a). 


\section{Changes to Family Roles}

In addition to health care needs there are other societal trends which have been recognised as influencing an older person's ability to age in place and live independently, notably changes to family structures and dynamics. Family structures, referred to here as the composition of kinship relations and family models, can vary in size and marital status e.g. lone parent households (Gillies, 2003; Schoeni \& Blank, 2000), and can potentially provide informal support to their older relatives, such as providing transport to medical appointments or social events (Musselwhite, 2011; Shergold \& Parkhurst, 2012). However, family structures have undergone radical changes in recent years for several reasons.

First, declining fertility rates (Office for National Statistics, 2017a; StatsWales, 2017a; WHO, 2015), coupled with increasing life expectancy resulting from improvements in public health, knowledge and medical innovation (National Institute of Aging, 2010; Vincent, 2006), have brought about population ageing, and a recognised imbalance in the ratios of older to younger age groups. Generally speaking, this means a decrease in the numbers of individuals in younger cohorts available to support older people formally or informally into later life (European Commission, 2006; WHO, 2015).

Additionally declining fertility rate and smaller household sizes in Wales (StatsWales, 2017b), indicates that family sizes have become smaller. Recent UK figures (covering England, Scotland, Wales and Northern Ireland) from the Office for National Statistics (2016) indicate that the number of one child families has increased (by 18.6\%), while the number of families with 2,3 or more children decreased between 1996 and 2016. Although the number of families overall has increased because different types of families (e.g. sole parents) are now recognised, and family sizes have become smaller (Office for National Statistics, 2016). It has been suggested that smaller family sizes mean that individuals may be unable to access reciprocal care arrangements or goods that have been typically associated with larger households (WHO, 2015) because there are fewer younger family members in place to provide care or support for their older counterparts (Hoff, 2015).

Although, international evidence suggests that the effect of increased life expectancy means that it is more likely that different familial generations will be alive at the same time (Bengston, Rosenthal, \& Burton, 1990; Grundy \& Henretta, 2006), this does not necessarily lead to a greater availability of family members to support older people 
due to the fragmentation of family structures (Hoff, 2015). Historically these changes have been attributed to increased geographic mobility. Through processes of industrialisation, traditional cottage based industries were replaced by factory based industries (Winstanley, 2011), and with it changes to residential mobility patterns as people began to relocate, sometimes on multiple occasions from their place of birth for work and education. Consequently families become more dispersed not only nationally but at times internationally (Burholt \& Sardani, 2018; Rainer \& Siedler, 2005). This also increases the risk of loneliness and isolation for older generations as broader family networks of support become sparse and the likelihood of older people living alone increases (Age UK Oxfordshire, 201 1; National Institute of Aging, 2010).

\section{Gender, Employment and Caring}

Changing gender norms and expectations about caring and paid employment have influenced the availability of family to provide care and support for its older members. The traditional role of women as caregivers to both children and aged family members has been shifting, as more women are engaged in paid employment (Chwarae Teg, 2013; Fernandez, 2007; WHO, 2015). This has resulted in fewer women of working age with the ability to provide informal care and support for their older relatives (Hoff, 2015; Timonen \& Doyle, 2008; WHO, 2015). This means that some aspects of independent living or ageing in place can be harder to achieve because complex negotiations over care and support arrangements may need to take place between family members, who must factor in work, other commitments, and geographic location (Rainer \& Siedler, 2005). As such, the family unit cannot always be relied upon to facilitate independent living and provide informal care and support to their older relatives (WHO, 2015).

In summary, like the other nations of the UK and beyond, Wales has experienced an increase in the number of older people who are living longer and remaining at home in the community, rather than living in institutional settings. However, welfare retrenchment in Wales has meant that many services which are fundamental to achieving independent living and ageing in place are becoming strained. This is because they must attempt to meet the complex, acute, and long-term health and social care needs of older people, notably when ageing is accompanied by healthrelated changes, co-morbidity and potential loss of mobility. These issues along with changing family structures (decline in fertility rates, verticalization of the family, increase smaller family sizes, geographic dispersion of family networks and the increase of women's participation in the work force), has meant that the nature and availability of informal support for older people to live independently in their own 
communities has changed. Furthermore, economic, health, socio-cultural and demographic transformations mean that for older people to age in place and live independently several challenges need to be overcome by the individual and the wider communities in which they live.

\section{Housing in later life}

Older people's housing tenure and housing conditions in Wales creates another element of complexity for achieving independent living through ageing in place. It is often stated that older homeowners prefer to live and remain in their family home until death (Ahlqvist et al., 2016; Gitlin, 2003; Sixsmith \& Sixsmith, 2008). However, there is a growing body of literature which challenges this assumption and is pushing towards a wider interpretation of what older people's "preferred" housing options are in later life (Costa-Font, Elvira, \& Mascarilla-Miró, 2009; Hillcoat-Nalletamby \& Ogg, 2014; Krothe, 1997). In Wales a recent report from the Expert Group on Housing an Ageing Population in Wales (2017) has recognised that some older people may prefer to have the opportunity to move home in order to meet their changing housing needs. This was also reflected by Hillcoat-Nalletamby and Ogg (2014) who conducted a national level survey to capture older people's subjective views of housing and neighbourhood environment. This article focused on dislikes of these areas and moving desired. The analyses found that $15.4 \%$ of the population surveyed were interested in moving to more suitable accommodation. Similarly, other studies have found that for residential mobility involves individuals having a choice of housing options (Burgess, 2012; Wiles et al., 2012).

Notwithstanding this debate, the most recent census data in 2011 indicates that ' $77.6 \%$ of people over the age of 65 in Wales owned or had shared ownership of their property' (Carter-Davies \& Hillcoat-Nallétamby, 2015, p. 7). Therefore, older people are predominantly homeowners, which can present certain challenges for those wishing to live independently in their own home. For instance being a homeowner entails maintenance, renovation and adaptation costs (Adams, 2010; Davey, 2006), but even if a dwelling is in a good state of repair homeowners can be faced with challenges to mobility, safety and security (Golant, 2008). This has led to some researchers expressing concern about the quality and suitability of the housing stock for ageing in place, which centres on issues pertaining to suitability, size and insulation (Adams, 2010; Golant, 2008; Watts, 2016).

\footnotetext{
${ }^{4}$ Figures were adapted using Office for National Statistics (2011).
} 
The availability of 'decent' housing stock will also determine the options that older have to age in place, and this varies considerably across regions of Wales. In an effort to combat this issue, the Welsh Government formulated the Welsh Housing Quality Standard which stated the vision of the Welsh Government that

'all households in Wales... shall have the opportunity to live in good quality dwellings that are... in good state of repair... Safe and secure, [and] adequately heated, fuel efficient and well insulated...' (Welsh Assembly Government, 2008, p. 2).

Research into the condition of Welsh housing stock estimates that $49 \%$ of the individuals surveyed, live in accommodation which fails to meet the necessary criteria of the decent housing definition ${ }^{5}$ or housing standards (Bibbings, 2017). Other studies have found that a third of the population of people living in non-decent accommodation are owned by an older person (Hamza \& Gilroy, 2011; Leather \& Morrison, 1997). Quite often this is due to poor thermal comfort, which is a result of outdated heating systems and poor insulation of the properties (Age Concern and Help the Aged, 2009). For instance, a report from Electrical Safety First (2018), found that almost one third of all domestic fire casualties (as a result of poor electrical safety and presence of safety hazards), are older people.

Other UK based studies suggest that issues associated with poor housing can contribute to other health related issues including compression of morbidity and experiences of co-morbidity (Chartered Institute of Environmental Health, 2008; Oldman, 2014; Roys, Davidson, Nicol, Ormandy, \& Ambrose, 2010). Older people are more likely to occupy homes that are in a poor state of repair, which can lack adequate bathroom or kitchen facilities and can be fuel poor including having inadequate or hazardous heating systems and insulation (Handler, 2014; Leather \& Morrison, 1997; Rudge \& Gilchrist, 2005). Poor housing is problematic because it can often be associated with negative physical and mental health outcomes (Krieger \& Higgins, 2002). For instance, the risk of winter deaths and falls can be closely linked to poor housing (Chartered Institute of Environmental Health, 2008; Handler, 2014; Mason \& Roys, 2011). Poorly insulated housing is a risk factor for some respiratory diseases ranging from asthma to COPD (Howden-Chapman, 2004; Lloyd, McCormack, McKeever, \& Syme, 2008; Rudge \& Gilchrist, 2005). The older population are most at risk because they tend to spend the majority of their time (80\% to $90 \%)$ indoors within their homes (Berke, Koepsell, Moudon, Hoskins, \& Larson, 2007; Handler, 2014).

\footnotetext{
${ }_{5}^{5}$ The Welsh Government formulated the Welsh Housing Quality Standard which stated the vision of the Welsh Government that 'all household in Wales... shall have the opportunity to live in good quality dwellings that are... in good state of repair... Safe and secure, [and] adequately heated, fuel efficient and well insulated...'(Welsh Assembly Government, 2008, p. 2)
} 
Throughout the UK there are regional differences in both the condition and energy efficiency of properties, but this variation is most prevalent when examining the housing stock in Wales. Overall, Wales has an older housing stock, which is in worse condition with high levels of disrepair (IpsosMORI, Shelter, \& British Gas, 2017; Welsh Government, 2014e) compared to the rest of the UK (Joseph Rowntree Foundation, 1997). Additionally, if the age of residences is used as an indicator of housing quality; the housing stock in Wales could be considered to be some of the worst in Europe because over one third of the houses in Wales were constructed before 1919 (Fisk, 1996; Fisk \& Hall, 1997). For instance in 2001, almost half (48\%) of the housing stock in Gwynedd (North Wales) was constructed before 1919 (National Assembly for Wales, 2001). An older housing stock is not necessarily problematic but, previous research suggests that older properties tend to have insufficient heating, poor insulation and have occupants who are fuel poor compared to newer builds (Healy \& Clinch, 2004).

In conclusion, Welsh Government is presented with challenges on several fronts when it comes to ensuring independent living for older people who are ageing in place. First, ensuring that there are public services in place to accommodate the increasing numbers and needs of older people who are living in their own homes; second, improving the housing stock to align with quality housing standards to overcome the barriers that can prevent older people from living independently and ageing in place; third, supporting informal carers or compensating for the lack of informal support for older people, resulting from changes to family structures and gender roles for women.

As this Chapter has shown so far, in Wales an unprecedented number of older people are home owners and are therefore confronted with the challenges of home maintenance. However, these challenges are often occurring in poor quality housing stock, making the fulfilment of ageing in place through independent living more difficult to achieve. This has created a need for services like Care \& Repair Cymru because they provide support for older people who may not have access to a network of family members; they take on and facilitate housing adaptation services and help clients to negotiate welfare application processes. 


\section{Care \& Repair Cymru: A Response to an Ageing Population and Housing Needs}

Since 1979, the Welsh Government has recognised and facilitated the work of Care \& Repair Cymru and its agencies whose central remit has been to deliver services to ensure that all older people across Wales 'can live independently in safe, warm, accessible homes' (Care \& Repair Cymru, 2016a, p. 3). Welsh Government's commitment to the organisation's work reflects a broader one of facilitating ageing in place, (Expert Group on Housing an Ageing Population in Wales, 2017; Hillcoat-Nalletamby \& Ogg, 2014), and collaborative and partnership working has been encouraged as a means of improving health, quality of life and services (Hayes, Mann, Morgan, Kelly, \& Weightman, 2012). Central to this has been an investment in person-centred, home-based social care and technical services provided through the national-level network of Care \& Repair Cymru (C\&RC) agencies.

As a charitable body, initially established in 1978, Care \& Repair Cymru define themselves as an 'older people's housing champion' (Care\&RepairCymru, 2015) and at the heart of their work they strive to ensure that older people are living in suitable accommodation which does not hinder them in their everyday lives. This overarching objective is achieved through its local agencies with a staffing team of Chief Officers, Technical Officers and Caseworkers.

Previously Care \& Repair Cymru had affiliate agencies in each of Wales' 22 counties, but recent funding restrictions and budget cuts have meant that $\mathrm{C} \& \mathrm{RC}$ agencies underwent an extensive structural reorganisation. At present there is an individual agency based in each county, but the services offered have Wales-wide coverage (Care \& Repair Cymru, 2017a). The structural reorganisation of the agencies has effectively meant that many Care \& Repair Cymru agencies must do more with less. While most have not experienced a dramatic reduction in the number of their front-line staff (Caseworkers and Technical Officers), the number of the management and support staff have been lowered to reduce costs, but there is still a requirement to meet the needs of older people (Care \& Repair Cymru, 2016a).

Care and Repair Cymru has several types of employees: Chief Officers, Technical Officers, and Caseworkers, which each have distinct roles. Chief Officers are the heads of the local Care \& Repair Cymru agencies who manage their staff and services (including social enterprise services). They work with regional and national bodies to create collaborations and to deliver services. Technical Officers are frontline service workers who work alongside the Caseworker service, aiming to provide 
expert advice and knowledge on technical aspects of housing issues. They also oversee building work on behalf of clients and act as a safeguard to ensure that structural changes adhere to building standards. The Caseworker role in Wales is supported mainly through core funding from Welsh Government. Caseworkers work on the ground with clients, meeting with them in their own homes, co-ordinating service packages and acting as link agents by connecting older people with other organisations. The nature of their work is multi-faceted and tailored to suit individual needs and can encompass a broad range of social care and repair services. For example, Care \& Repair Cymru agency Caseworkers can undertake financial checks to ensure an older person is receiving the correct level of publicly funded financial assistance (e.g. attendance allowance or winter fuel allowance). Equally, Caseworkers can facilitate more large-scale housing adaptation or building work such as conversions, or extensions.

From this brief overview, it appears that agency Caseworkers potentially play not only a central role in assessing and addressing client needs across Wales, but also have the capacity to generate and facilitate high levels of collaborative and partnership working. From the service initiative examples given, they are playing a pivotal role in stimulating partnership working between local government authorities, practitioners (e.g. social workers), third sector, and business sector service providers in meeting client requirements.

As outlined in the introductory chapter, an exploratory review of the literature at the outset of the research showed that there is to date, little understanding of the increasing complexity of the Caseworker role and the networks they develop in response to diverse client needs, in a context of economic recession and reduced government spending. Much current research has tended to examine the actions (Adams, 2017; Mackintosh \& Leather, 2016; Mynatt, Melenhorst, Fisk, \& Rogers, 2004), strategies (Care \& Repair England, 2013; Dupuis-Blanchard et al., 2015), or services (Adams, 2018; Anonymous, 2011; Croucher \& Lowson, 2011; Davidson, 2012) that help older people to stay at home and age in place. For example, research has examined how their homes can be adapted or retrofitted to changing health and mobility needs (Powell et al., 2017). Other studies have evaluated home repair, maintenance and handyperson services in England and Wales to determine how effective they are in facilitating independent living and ageing in place (Adams, 2018; Anonymous, 2011; Croucher \& Lowson, 2011; Powell et al., 2017). Finally, studies have also examined the strategies or initiatives put in place to achieve ageing in place (Auditor General for Wales, 2018; Davidson, 2012; Dupuis-Blanchard et al., 2015), 
as well as the views of older people about the importance and meaning of home (Care \& Repair England, 2013).

What these studies have in common is that they focus on the things that can be done to promote independent living through ageing in place, rather than on the people or workforces who ensure that these things are coordinated and put in place to achieve this objective. The PhD will address this imbalance by exploring Wales' Caseworker workforce as their potential to act as link agents in terms of their ways of networking and linking with others, the skills and expertise they bring to bear on this and their potential to promote cross-collaborative partnership working.

\section{Chapter Summary}

To summarise, this chapter has explored the Welsh context in which the thesis is grounded. Beginning with an exploration of the population of older people in the UK and Wales, it is evident that the number of older people is increasing and that the population of older people is itself ageing. Additionally, the policy response made by the Welsh Government to the ageing population has been shaped by notions of ageing in place and independent living, and key to this is the effective delivery of services. This chapter then addressed issues that can impede independent living and ageing in place including the condition and suitability of the housing stock, decreased public spending in health and social care sectors, the increasing complexity of health care needs of older people, and changes to family roles and obligations. The interconnectedness of these issues can interact make ageing in place more difficult to facilitate in practice. Finally, Care \& Repair Cymru and the services they offer facilitate independent living and fill the gaps caused by cultural, social and demographic changes. Chapter Two builds on this chapter by examining theoretical literature which can be used to examine service delivery and understand the Care \& Repair Cymru agency Caseworkers. 


\section{CHAPTER TWO}

\section{Literature Review}

This chapter reviews academic literature and social theories that have shaped the research design, methods of data collection, formulation of research questions and the interpretation of the findings. The literature review method is discussed, followed by a critical overview of the theoretical foundations of the thesis including theories of social capital, human capital, network theory and emotion work. The chapter then moves on to reviewing social network analysis as a methodological tool and provide an appraisal of studies relevant to the $\mathrm{PhD}$ which have applied this methodology. A summary of findings from the literature review and how the social theories will be used in this thesis then follow. The chapter concludes by elaborating a preliminary conceptual framework of the Caseworker role, in addition to outlining the research questions informed by the review.

\section{Approach to Reviewing the Literature}

The literature review for this thesis had an initial starting point which was social network analysis. The rationale for this stemmed in part from the origins of the research which evolved from two Strategic Insight Programme grants awarded to Dr Hillcoat-Nallétamby. These enabled the co-production of a broad research project which reflected a strategic objective identified by Care \& Repair Cymru - establishing a better evidence base about the role Caseworkers play in delivering services to older people across Wales. A preliminary reading of literature around social network theory, analysis and empirical studies highlighted the relevance of social and human capital theory. The significance of emotion work theory became apparent once some of the initial fieldwork had been completed with Chief Officers who recognised an affective dimension to the Caseworker role.

The thesis adopted a narrative literature review approach. Fundamentally, this approach involves reviewing academic literature to interpret and critique current and previous research with the aim of reaching a deeper understanding of relevant topics or themes (Cronin, Ryan, \& Coughlan, 2008; Greenhalgh, Thorne, \& Malterud, 2018; Jahan, Naveed, Zeshan, \& Tahir, 2016). It is particularly useful when attempting to link together research from a variety of theoretical or contextual perspectives to generate or develop social theory (Baumeister \& Leary, 1997; Bryman, 2008). This means that narrative literature reviews are not limited to one type of research field or publication. They can be contrasted with a systematic review which uses a more 
structured and selective approach to reviewing literature. Typically, an exhaustive search strategy is used which produces an initial body of several hundred or thousands of potentially relevant studies. These studies are then whittled down using explicit inclusion and exclusion criteria to extract the most relevant or most useful before a researcher considers what they mean collectively (Greenhalgh et al., 2018). Systematic reviews are designed to seek to address pre-determined and focused research questions (Cronin et al., 2008), by providing a summary of pre-existing data and findings which can be replicated (Bryman, 2008; Greenhalgh et al., 2018).

Narrative approaches to reviewing literature have shortcomings, notably if they are carried out with a highly selective focus (Baumeister \& Leary, 1997); in other words, researchers may cherry pick their evidence to support a particular interpretive approach (Baumeister \& Leary, 1997; Greenhalgh et al., 2018). Furthermore, because narrative reviews of the literature tend to accompany constructionist approaches to social research, emphasis is placed on developing a deeper understanding rather than generalisations about social phenomena (Greenhalgh et al., 2018). Finally, there also appears to be no consensus or clear manner of undertaking a narrative literature review which means that the manner in which they are undertaken can vary significantly (Baumeister \& Leary, 1997; Greenhalgh et al., 2018).

Despite these shortcomings, utilising a narrative approach was considered to be suitable for this thesis because it would enable the identification of sources on a broad range of research topics, theories and methodologies from different disciplinary fields. The review was exploratory and led to the identification of research questions which were grounded in an examination of relevant studies, theories and the local social context. A systematic review of the literature would not have been appropriate because the research questions were formulated after the literature was considered and reviewed (Wright, Brand, Dunn, \& Spindler, 2007).

\section{Process}

Several databases were searched including Scopus, ProQuest, Web of Science, Google Scholar, Stats Wales, Welsh Government and Swansea University's iFind database and library catalogue. Quality of sources was judged based three factors: the number of times a source had been cited, if the paper was peer-reviewed and if reports had been commissioned by governmental departments. Reviewing the literature was a reiterative process involving many stages of searching, reviewing and refining results and continued throughout the data collection, analysis and the writing up of results 
(see Figure 2.1 which is adapted from Brereton, Kitchenham, Budgen, Turner, and Khalil (2007); Hjermstad et al. (2011); Kannisto, Koivunen, and Välimäki (2014)).

The focus was on identifying existing studies which combined topics on social network analysis, ageing in place, workforce, community and service delivery, in addition to exploring the social, policy and demographic context in which Caseworkers were operating. Searches were limited to English language materials and some exclusion criteria were set (e.g. when examining applied social network analysis literature, studies exploring online networks were excluded because online communication and networking is different from that of face-to-face networks). No time span was specified because some of the social theories were developed in the early 1960 s and it was therefore important to access these early works in addition to contemporary revisions of these theories to ensure that recent developments were considered and incorporated into the theoretical frameworks and methodological design of this thesis.

Figure 2.1- Illustration of the processes involved in the review of the literature

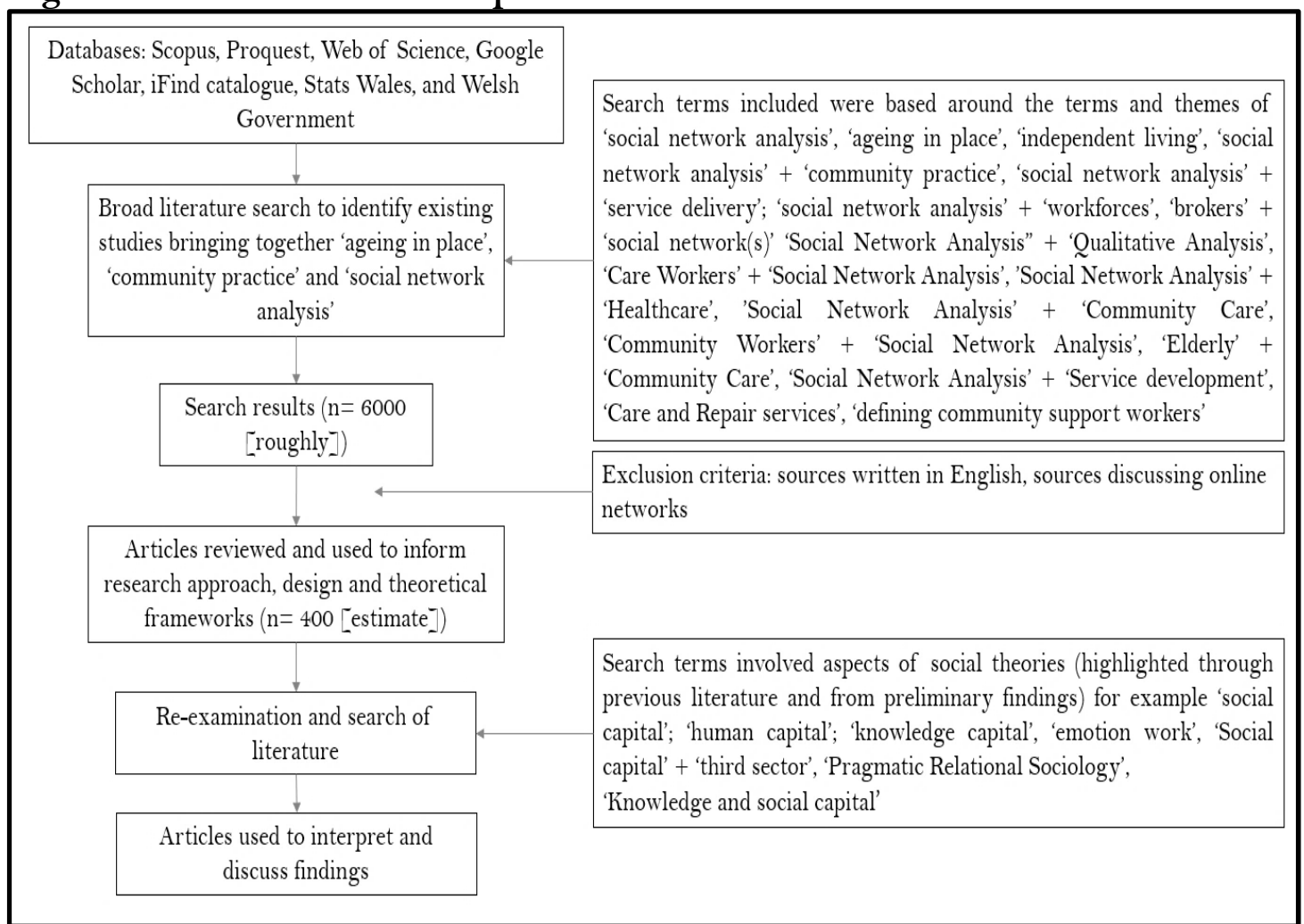

Findings were used to inform the methodological approach and design of the research tools. The theories used were influenced in part by the findings from the data collection and from a review of previous studies and academic literature. 


\section{Critical Overview: theoretical foundations of the thesis Social Capital: Bourdieu, Coleman and Putnam}

Social capital theory enables an exploration of the attributes that Caseworkers as individuals and as a workforce bring to their roles. Some scholars associated with this field view social capital as an individual asset, with others seeing it as a collective asset. Three classical scholars, Bourdieu, Coleman and Putnam, are recognised as having laid the foundations of discussions around social capital.

Bourdieu's theory of social capital is heavily influenced by Marx's concept of capital and its conflict perceptive, and is a prominent feature of his exploration of social class and inequality and the role of cultural symbols in reproducing inequality (Field, 2003; Lin, 1999; Siisiainen, 2003). From this perspective, capitals are viewed as entities that can be accumulated, exchanged and drawn on to produce profit (Field, 2003; Lin, 1999). Bourdieu views social capital as class-based and as a resource like economic (financial) capital and cultural capital (education and taste), which can be drawn upon to advance a person's interests and social position but will ultimately result in inequalities (Field, 2003). According to Bourdieu, social capital is:

'...the sum of the resources, actual or virtual, that accrue to an individual or group by virtue of possessing a durable network of more or less institutionalised relationships of mutual acquaintance and recognition' (Bourdieu \& Wacquant, 1992, p. 119).

Therefore, social capital can enhance a person's position in a network or the social structure in which they are embedded. This improvement in social position can occur despite the lack of other resources such as economic and cultural capital because social capital has the potential to complement or at times be a substitute for other resources (Adler \& Kwon, 2002; Field, 2003). Social capital also entails aspects of honour and respectability, which can serve as currency in advancing social position (Field, 2003). Furthermore, Bourdieu contends that the amount of social capital that a group possesses is a result of a combination of network size and the volume of different forms of capital (cultural, symbolic and economic) that networked individuals have (Lin, 1999).

Another theory of social capital is derived from the work of Coleman who defines social capital by its function and uses it to explain collective action and co-operation within the framework of rational choice theory (Field, 2003). Rational choice theory assumes that human behaviour is highly individualistic, where a person's automatic behaviour will reflect actions that will best serve their own interest irrespective of the outcomes for others. Coleman's version of social capital theory was influenced by Becker (1962), and his work on human capital (the education and training that individuals and organisations invest in to gain advantage in work markets), and he 
applied it to examine academic achievement in schools. Coleman argued that the most important factor in explaining different patterns of school attendance and educational attainment was the influence of community norms. These norms reinforced teachers' expectations and Coleman therefore concluded that communities were a source of social capital which could counteract the effects of social and economic disadvantage within families (Coleman, 1988; Field, 2003), leading to the development of human capital.

Like Bourdieu's conceptualisation, Coleman sees social capital existing within the structure of relationships. However, he does not adopt a conflict perspective, but rather views social capital as a means of facilitating social action which has two essential components: social structure (e.g. obligations, expectations, trustworthiness, information channels and norms and effective sanctions) and the ability to facilitate specific actions of actors (individuals or groups), while inhibiting others. In other words, social capital is a neutral resource that can be accessed by anyone in a community to facilitate social action (Adler \& Kwon, 2002) through networks or relationships of norms, trust and reciprocity (Coleman, 1988; Nahapiet \& Ghoshal, 2000). However, whether this social action is beneficial or advantageous to communities or individuals depends on the context in which it is based.

In sum, Coleman viewed social capital as an asset for individuals which is constructed from structural attributes or resources (Coleman, 1990). Some of these incorporate expectations of reciprocity and therefore involve relationships that are shaped by shared values and a sense of trust (Field, 2003).

An alternative theory was developed by Putman, who conceptualised social capital as a feature of social organisations. Specifically he sees social capital as the 'networks, norms, and social trust that facilitate coordination and cooperation for mutual benefit' (Putnam, 2000, p. 2). Putnam's theory of social capital has similarities to Coleman's, in that social capital is an asset which can be used to facilitate social action. However, Putnam examines the role of social capital in civic participation, volunteering, community and democracy (Grossman, 2013; Putnam, 2000; Putnam et al., 1994). In contrast, Bourdieu explored the role of social capital in producing and reproducing social inequality, while Coleman used it to explain collective action. Putnam views social capital as a characteristic of organisations or a public good which should be inherited and maintained over time (Grossman, 2013; Putnam, 2000). A key indicator of this form of social capital is trust, with higher levels of trust in organisations indicating that a region has high levels of social capital (Putnam et al., 1994). 
Consequently, areas with high levels of civic engagement and the successful performance of political systems can be attributed to the successful accumulation of social capital (Putnam, 2000; Siisiainen, 2003). On the other hand, social problems can be failure to secure and accumulate social capital over time (Siisiainen, 2003).

\section{Neo-capital theorists}

In addition to the three classical theories of capital, more contemporary neo-capital theorists (Adler \& Kwon, 2002; Burt, 1997; Flap \& Völker, 2013; Lin, 1999, 2001) have further developed the meaning and function of social capital. Whilst they use and understand social capital in slightly different ways, they can be grouped together by the common idea that social capital is a privately held resource, or an individual asset. Rather than seeking to explore social order in terms of the role of social capital in reproducing or overcoming inequality (in line with Bourdieu and Coleman), neocapitalists understand social structures as encompassing different layers of nuanced debates, discussion and interaction. Individuals can then draw upon their social capital to negotiate these discourses to facilitate action and generate expected returns (Lin, 1999, 2001). For example Lin $(1999,2001)$ states that having the ability to access and utilise social capital can lead to improvements in an individual's social position. If individuals can access a wide range of resources including knowledge, and social contacts they can use these aspects to improve their own social position.

Additionally Burt (1997) asserts that occupying certain position in networks near structural holes ${ }^{6}$ can impact on individuals obtaining better positions i.e. job roles in organisations. Specifically, this occurs when two individuals are located near structural holes but hold complementary information. Due to the different connections that individuals obtain, certain individuals can occupy pivotal positions as brokers and can facilitate the flow of information across boundaries or gaps in networks to help improve individual's social positions (e.g. new job roles). From an individual level conceptualisation, social capital is primarily understood to be the 'resources embedded in a social structure which are accessed and/or mobilized in purposive action' (Lin, 1999, p. 35). Therefore, social capital refers to an investment made by individuals in social relationships which can be accessed to achieve a goal or some form of advantage during a time of crisis, for material gain, personal advancement,

${ }^{6}$ This is a theory which brings together both network theory and social capital. It posits that social structures are characterised by clusters of dense connections. These clusters contain homogeneity of ideas, behaviour and information and a structural hole represents a gap between the clusters of relationships. Social capital represents opportunities to act as a broker or bridge between these clusters to bring about advantages for instance access to new information (Burt, 1997). 
enjoyment, or to mobilize collective action (Lin, 1999; Woolcock \& Narayan, 2000). The effects of social capital can range from the flow of information and influence, to mobilizing social action (Adler \& Kwon, 2002).

Studies on the links between social capital and social networks provide additional definitions of how social capital can be built and utilised in social networks. For instance, Woolcock and Narayan (2000) assert that social capital is wisdom born of experience' (225-6), and Hawe and Shiell (2000) postulates that social capital requires an investment of energy and time to maintain the networks that underlie social capital. This is also reflected in the work of Portes (1998), who states that 'social networks are not a natural given and must be constructed through investment strategy orientated to the institutionalization for group relations' (p.3).

Studying human capital in the field of economics Glaeser, Laibson, and Sacerdote (2002), assert that what classical theorists consider to be social capital (norms and networks of reciprocity etc.) are actually reflections of what communities do rather than the capital that people own. Instead social capital should reflect an individual's intrinsic attributes such as social skills, charisma, and the networks that have been developed (Glaeser et al., 2002). While the intrinsic attributes of individuals or social skills (Bowles, Gintis, \& Osborne, 2001) can represent the 'social component of human capital' (Glaeser et al., 2002, p. F438), the authors assume that an individual's attributes and their social networks are indistinguishable from each other and therefore represent individual social capital.

There are several critiques and shortcomings of social capital theories. Haynes (2009, 2014) considers the language of capital to be inappropriately applied to the study of interpersonal relationships. This is because such studies create a false comparison between personal relationships and economic exchanges found in capitalist markets (Haynes, 2009). Writers who adopt this position view social capital as a reductionist theory which removes the social aspect of inter-personal relationships (Cohen, 1999; Field, 2003). Another critique of social capital is that the negative impacts of social capital for individual and community status or cohesion are largely glossed over (Field, 2003). For instance, Coleman almost unilaterally discusses social capital as a means of overcoming barriers and increasing human capital. On the other hand, Putnam does acknowledge that there are negative consequences to social capital, but these are not really discussed in much detail. Bourdieu is perhaps the most critical because social capital is conceptualised as an asset of the privileged individuals, but it 
is only considered to be negative for those who are in a disadvantaged position (Field, 2003).

Furthermore, there appears to be a lack of consensus amongst social capital theorists as to whether it is viewed as a group or individual level asset (Daly \& Silver, 2008; Field, 2003; Lin, 1999). Haynes (2014) criticises both Bourdieu and Coleman for being ambiguous in discussing social capital as both group and individual level assets. In contrast, neo-capital theorists and Putnam understand it to be an individual level asset. This lack of consensus on a conceptual definition of social capital makes its use as a theoretical framework much more complicated.

A final critique is that the potential impact of 'social skills' on social capital is largely ignored. For instance, while Coleman (1988); Putnam (2000); Putnam et al. (1994) examined social processes or structures in relation to the development and function of social capital; most theorists make little reference is made about the social skills or characteristics.

\section{Human capital}

Human capital theory can be traced back to the work of Adam Smith (Spengler, 1977) but was popularised in the 1960's by Schultz (1961) and Becker (1962) when examining the growth of income in some countries which could be attributed solely to physical resources (Chartered Institute of Personnel and Development, 2017).

Human capital can be defined as the stock of skills, knowledge and abilities acquired by individuals that can be developed through experience, education and training (Becker, 1962, 1994; Coff \& Kryscynski, 2011; Coleman, 1988; Schultz, 1961). This conceptualisation has been widely used in economic or business studies where it is viewed as a value or costs to an organisation, and the accrued skills, experiences and knowledge of workers that can be exploited or used to generate returns or some form of advantage (Israelsen \& Yonker, 2017). For instance, the migration of younger workers from rural to urban areas or less developed countries to more developed nations, and the resulting impact on economies and businesses has been seen as a consequence of the loss of human capital in some areas as individuals seek to advance their careers and marketable skills (Beine et al., 2008). On the other hand, employers investing in the human capital of their workforces (through training and education) are likely to generate a greater level of returns (e.g. increased social capital or profit) because the workforce individuals will be engaged in work for a longer period of time. 
There are some limitations to human capital theory which stem from the assumptions underpinning it. Sweetland (1996) has suggested that there is a paradox translating micro or individual processes (i.e. investments in education and training) to the macro (or societal level), and that human capital theory does not acknowledge its own short comings. For instance, if education is equated with higher income and better opportunities, there is a lack of an answer to why some educated individuals are unemployed, why the standard of living has not improved and why some economies stagnate despite experiencing an increase in educational attainment (Sweetland, 1996). Similarly Piketty (2014) notes that one of the assumptions underpinning human capital theory is that other forms of capital i.e. social and financial have diminished and are not as important in determining social mobility. This assumption makes the relationship between education, work, earning potential and social mobility linear and oversimplified (Piketty, 2014). Another recognised limitation of human capital theory is what Tan (2014) refers to as 'methodological individualism' which assumes that the roots of social phenomena can be traced back to individual behaviours. In other words, human capital reflects the behaviours that individuals undertake to maximise their own interests and gain advantageous social positions e.g. investing in education to access well paid occupations. The limitation of this assumption is that it fails to recognise organisational decisions regarding investing in training courses for their workers (Tan, 2014). However, not all proponents of human capital theory adhere to this assumption.

Also, the paradigm of rational choice theory has typically underpinned human capital theory. As outlined in the social capital section, rational choice theory is a model which enables researchers to predict and understand human behaviour (Tan, 2014). As with methodological individualism, human capital is seen as an effective way of improving one's social position because specialist education or training can enable individuals to enter desirable and well-respected occupations. The limitation of a rational choice approach is that social or contextual aspects are not acknowledged (Piketty, 2014). For instance, it could be argued that familial or social factors influence education and training choices, rather than an individual's drive to obtain certain social positions through occupations and earning potential (Tan, 2014).

\section{The Network Perspective: theory and method Network theory}

Previous studies (Ennis \& West, 2010; Inkpen \& Tsang, 2005; Lin, 1999; Mobius, Quoc-Anh, \& Rosenblat, 2004) have indicated that there appears to be an association between social capital and social networks. As Ennis and West (2010) note: 
'The links between individuals and organisations are key to sharing of information and resources, and mobilisation for change. Social networks are a central concept in social capital literature and are generally considered to be the 'structure' within which social capital can exist' (Ennis \& West, 2010, p. 408).

Using this conceptual definition of social capital along with the previous examination of capitals, it is clear why many studies examining social networks make reference to social capital. Not only are social networks the structure in which social capital is able to exist, but network theory provides a means of explaining the nature and dynamics of relationships. For instance, because social capital is dependent upon social relationships and networks, the more relationships that individuals acquire and maintain, the larger one's networks and social capital appears (Smith et al., 2006). In other words, for social capital, social networks are important resources (Field, 2003) and vice versa.

The basic premise of network theory (sometimes referred to as social network theory and the network perspective) is that any system (e.g. society or organisation) is conceptualised as a group or network of interrelated entities or individuals (Borgatti $\& \mathrm{Li}, 2009)$. It is a structural approach which aims to capture information about the patterns of relationships or links between entities and to study these complex interacting systems by representing them as graphs (Baez, 2016; Luke \& Harris, 2007). It regularly utilises mathematical and computational models and is consequently a highly graphical theoretical approach to social research (Luke \& Harris, 2007).

Network theory operates on the assumption that organisations and individuals are rooted in a 'relational structure' (Wäsche, 2015) and consequently 'concerns itself with relational data and research questions'(Luke \& Harris, 2007, p. 70). Network theorists therefore assume that individuals do not exist in isolation, but rather, are connected and form relationships or links with each other. Another assumption of the theory is that networks are not randomly generated; instead they are created using certain rules or are defined due to how elements choose to interact with each other and other networks. In other words, individuals act or react in relation to other actors found within a network (Laumann, Marsden, \& Prensky, 1989; Wäsche, 2015).

However, network theory has recognised shortcomings. One limitation is that while it is useful for mapping and examining relationships between entities, it fails to contextualise them. Additionally, another criticism of network theory is the 'boundary specification problem' (Yang, Keller, \& Zheng, 2017, p. 28); this refers to the problem of where the boundary of the network or set of relationships should lie. Another 
dimension to the boundary specification problem is that network theory can be blinkered and does not take into account how individuals outside of a network can influence (De Brún \& McAuliffe, 2018). There is potential for individuals outside of a network to have an impact the dynamics or the spread of knowledge, ideas and actions through a network of relationships, yet this is not acknowledged in social network studies.

\section{Social Network Analysis}

Network theory is closely intertwined with but different to, social network analysis. If network theory can best be understood as a relational based theoretical approach to studying groups or organisations, social network analysis (or structural analysis as it is sometimes called) on the other hand, is not strictly speaking a formal theory but rather an analytical tool or 'broad strategy for investigating social structures' (Otte \& Rousseau, 2002, p. 441). In sum, the network theory is operationalized, measured and visualized using social network analysis. Social network analysis uses a set of methodological tools to gather relational data from an organisation or group of individuals (Provan, Veazie, Staten, \& Teufel-Shone, 2005; Yang et al., 2017) to examine how they are connected (Ennis \& West, 2013). It finds its origins in mathematics and graph theory, dating back to the 1930s, and has since been adapted by the social sciences. Working from the basic assumption that a social network is like a social map then a social network can represent four different things: a conceptual model, a description of an existing real-world structure or system, a mathematical model, or a simulation (Luke \& Harris, 2007).

Social network analysis operationalizes network theory by producing diagrammatic representations of relationships between individuals (sociograms) which may be otherwise difficult to represent. A sociogram is a visual representation of an individual's 'patterns of daily living and, in particular, the people, places and activities that are significant... a network is like a map' (Seed, 1990, p. 30). In other words, social network analysis generates visual data that illustrates connections between individuals or organisations or lack thereof. Figure 2.2 is an example of a sociogram that can be generated using social network analysis to illustrate the connections between a group of individuals (Seed, 1990). Diagrams of this nature are the starting point of many social network analysis studies. Starting with a sociogram, a researcher will continue their analysis by determining who is connecting with whom and who could be 'important' or hold strategic positions within that network. 
Figure 2.2- Simple 'Star network’ sociogram. Adapted from (Seed, 1990, p. 10).

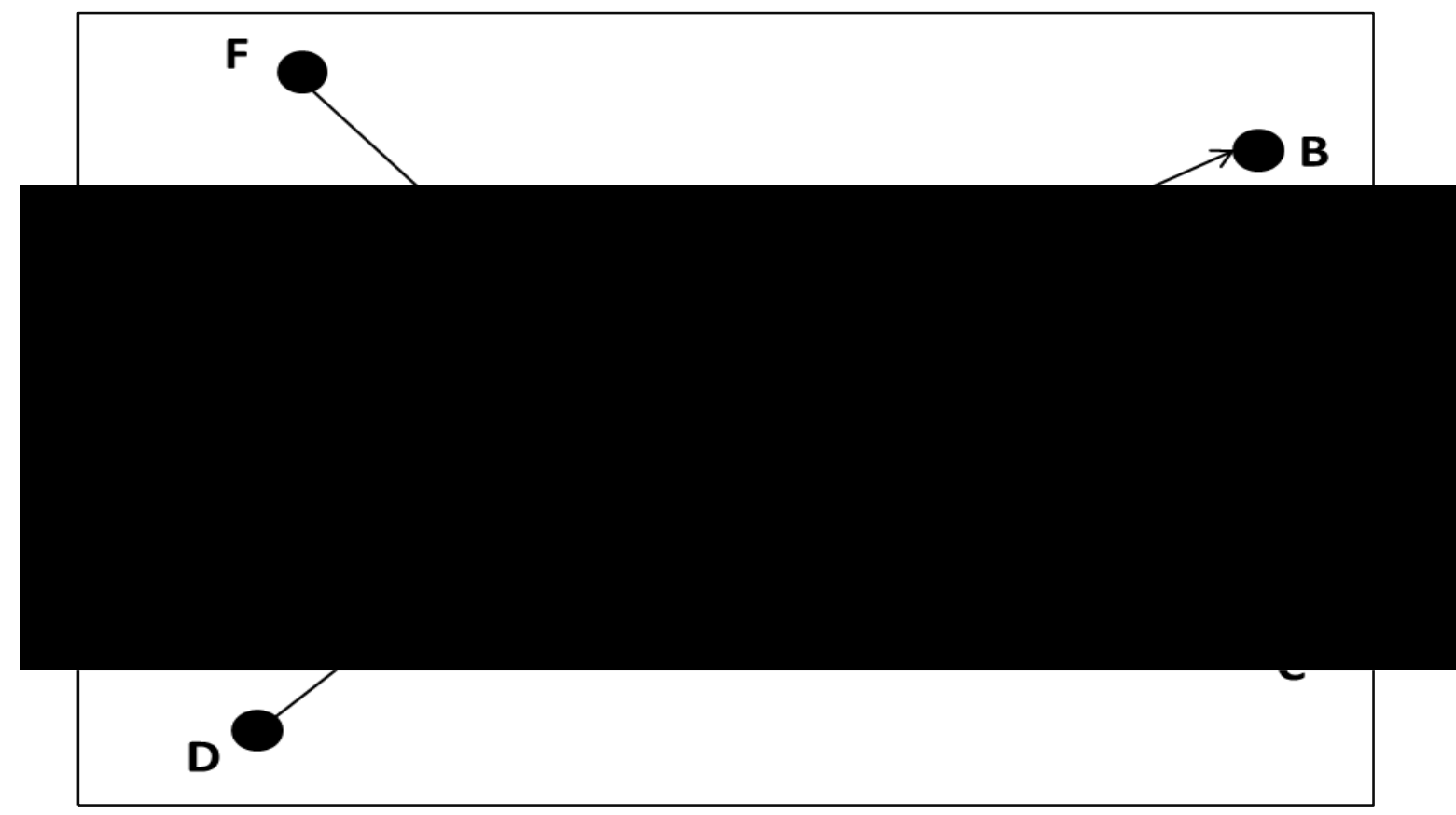

For instance, Figure 2.2 illustrates that there are six actors in within the network. Not all the individuals link with each other. Actor A has and makes the most connections (5 incoming and 2 out-going connections) and is therefore at the centre of the star network. Actors $\mathrm{B}$ and $\mathrm{C}$ have the second highest number of connections ( 1 out-going and 1 in-coming each), however Actors D, E and F make only one connection. Overall, a star network indicates that there is a relative inequality in a network, because one or a few individuals make more connections than others which can impacts aspects such as power (Hanneman \& Riddle, 2005), or the spread of innovation and knowledge across a network (Fleming \& Waguespack, 2007; Sibbald, Wathen, Kothari, \& Day, 2013). By analysing relationships which are embedded in a comparatively larger social structure, a researcher can '...tie together so many interdependent parts that constitute micro- and macro- social orders' (Galaskiewicz \& Wasserman, 1994, p. xiii).

Although social network analysis is useful for illustrating the connections between individuals or agencies, examining the structure alone only gives a partial understanding of the dynamics of the network (Provan et al., 2005). Furthermore, as a tool it only maps a social structure at one point in time (Wey, Blumstein, Shen, \& Jordán, 2008). The relationships and networks that individuals and organisations develop, are not static but constantly evolve, therefore social network analysis only provides a 'snap shot' in time of a network or a structure of relationships. This is a shortcoming because any potential findings or recommendations made could be redundant by the time results are published or disseminated (Mislove, Koppula, 
Gummadi, Druschel, \& Bhattacharjee, 2008; Mislove, Marcon, Gummadi, Druschel, $\&$ Bhattacharjee, 2007). However, this does not mean that the findings are not valid because this approach can still generate an insight into how organisations work to achieve certain goals (Cross et al., 2002).

In sum, social network analysis describes a set of methodological tools which can be used to examine and explore the relationships between individuals and between organisations. Studies relevant to the thesis which have applied social network analysis techniques will now be reviewed.

\section{Applied social network analysis studies}

Social network analysis has been applied to several areas of study (Vassilev et al., 2013). These include organisational management, collaboration and knowledge sharing (Cross et al., 2002; Liebowitz, 2005; Tichy et al., 1979; West \& Barron, 2005; West, Barron, Dowsett, \& Newton, 1999; Wey et al., 2008); online networking (Caulfield, 2013; Garton, Haythornthwaite, \& Wellman, 1997; Mislove et al., 2007); crime prevention (Carrington, 2011; CGI Group Inc, 2011; Colladon \& Remondi, 2017; McIllwain, 1999; Xu \& Chen, 2005); the use and uptake of medical innovations amongst health professionals (Coleman et al., 1957; Van den Bulte \& Lilien, 2001; West et al., 1999), and service delivery and development (Nicaise et al., 2012; Woodcraft, 2011). However, few studies have adopted this analytical tool to conduct an ego-centric ${ }^{7}$ examination of the networking activities of a highly specialised workforce such as Caseworkers in a third sector organisation. Social network analysis in this area of research is therefore still in its infancy; however, the materials reviewed in the following sections illustrate recent studies which have examined workforces in different ways. Despite the focus of the studies differing from that of this thesis, the studies remain relevant and have informed the research design and interpretation of findings in different ways.

Traditional social network studies tend to focus 'on the relationships and interactions of the members (actors) rather than their individual attributes or behaviours' (Long, Cunningham, \& Braithwaite, 2013, p. 159), but other studies (Coleman et al., 1966; Van den Bulte \& Lilien, 2001; West et al., 1999) have broadened this scope of research to also consider contextual factors. Contemporary users of social network analysis and the network perspective emphasise that researchers need to move the analysis

\footnotetext{
7 This term will be defined and explored in greater detail in Chapter Four, but briefly an ego-centric examination means that the focus of a study is on an individual or set of individuals within a wider social structure and the relationships they have (Seed, 1990).
} 
beyond 'dyadic processes of transaction to capture the complexity of managing... services' (Wäsche, 2015, p. 544). While Wäsche (2015) examined sports services, his approach and use of the network perspective is applicable to other areas of research in which the management of services is a part of the focus. Studies by Nicaise et al. (2012) and Woodcraft (2011) have used social network analysis to explore and identify different roles that organisations and individuals have undertaken within healthcare and community service networks in U.K and European contexts. These studies indicate that organisations and individuals occupy different roles in these networks, for example as hubs (people who have the greatest number of direct connections to others), gatekeepers or brokers (individuals who occupy pivotal positions between hubs and can act as bridges between disparate parts of networks), pulse-takers (the opposite of hubs but have the most influence using the smallest number of direct ties) and $c o-$ ordinators (individuals who act in a brokerage capacity to organise and manage service delivery) (Nicaise et al., 2012). These individuals work in subtle, indirect ways to shape the dispersion of knowledge, ideas and actions through a network (Stephenson, 2005; Woodcraft, 2011).

The studies conclude that the function and importance of these roles needs to be understood and utilised correctly to ensure that people's needs are addressed and that services are delivered efficiently. In the context of this thesis, the evidence has highlighted the importance of exploring where Caseworkers operate within the Caseworker network, and the different kinds of links they make with their colleagues, external organisations and clients.

West and colleagues' study focused on network measures including density (how many people are contained in a network), centrality (how many connections each individual makes and receives) and centralisation (the equity of connections amongst individuals in a network). This study found that these factors represent the professional socialisation and structural location of individuals in networks and should be considered in designing quality improvement teams (West et al., 1999). Other studies (Coleman et al., 1957; Van den Bulte \& Lilien, 2001), have found these measures to be linked to factors including social control, social influence and access to information.

Other studies also explored the context in which social networks are based. These have been reviewed below and have informed the approach that this thesis adopted in collecting contextual data about local and national contexts in which the Caseworkers were operating. 
Van den Bulte and Lilien (2001) used social network analysis to explore the idea of social contagion in social networks (the spread of behaviour or ideas, from one person in a group to another (Marsden, 1998), specifically in relation to the adoption of a drug by medical professionals. The authors also re-examined the original work published by Coleman et al. (1966) to explore and empirically demonstrate that social network effects may be 'confounded with common contextual factors' (Van den Bulte \& Lilien, 2001, p. 1411). Both Van den Bulte and Lilien (2001) and West and Barron (2005) highlight the importance of recognizing the context in which studies are based.

Finally, other studies have deviated from a traditional social network approach to examine how individual characteristics can influence social networks. Provan et al. (2005) used social network analysis to link theory and practice by demonstrating how social network analysis can be used to build community partnerships. The results indicated that by tracking relationships amongst organisations in networks, the leaders of these organisations can build capacity and address the needs of the communities that they are serving. Whilst Provan et al. (2005) acknowledges that relationships between actors can reflect wider societal structures, they also argue that to ignore the attributes or qualities (social and human capital) that an individual accumulates would miss a vital component of relationship building:

'Network analysis is useful for demonstrating the connections and relationships among agencies, reflecting the structure of the network. But, structure alone provides only a partial understanding of why a network may or may not be effective' (Provan et al., 2005, p. 605).

Meltzer et al. (2010) explored the applicability of individual social network measures to an academic healthcare context in Chicago. The result from this study indicates that new measures examining group level attributes can identify an optimal group of individuals to target when seeking to disseminate information and improve the quality of services within a health care setting.

Overall, these studies suggest that social network analysis is a useful tool to capture how Caseworkers are interacting with colleagues and other organisations to deliver services. Furthermore, they demonstrate the importance of considering the context in which the networks are based to give a more complete understanding of how networking and interacting is taking place. 


\section{Emotion (al) Work}

While capital theories and social network analysis are suitable theoretical and empirical tools for examining the Caseworkers as a workforce the attributes they bring to their roles and how these attributes have the potential to impact service delivery, there are certain aspects of the role which these theories fail to capture, notably the more affective dimensions.

The nature of their role requires Caseworkers to meet face-to-face with clients and work with them to deliver tailored services and solutions. This aspect of their role has already been shown to entail the use of emotional skills (Hillcoat-Nalletamby \& Sardani, 2015). It is important to acknowledge and explore this facet of the Caseworker role as this represents a key aspect of the reality of their work which is not referenced in their job description, and as such may be overlooked. The affective dimension of service delivery has been recognised in nursing research (Allan, 2006; Bolton, 2000, 2001; Bone, 2002; Gray, 2009; McQueen, 2004), which has shown that these aspects were tacit and sometimes unrecognised skills but were vital in establishing good professional-patient relationships. Similarly, studies in care work (Aronson \& Neysmith, 1996; Cancian, 2000; Lopez, 2006; Treweek, 1996) have also acknowledged the complex emotional processes that care workers undertake to support their clients.

The review of literature around emotions led to the identification of three prominent theories which are used in research to examine the affective dimension of some workforces. These include emotion work, emotional labour and emotional capital.

As a concept, emotion work describes the processes undertaken by certain individuals to enhance the well-being of others (Strazdins, 2000). These actions include, but are not limited to having empathy, building positive and co-operative relationships with others, and providing emotional support (England \& Farkas, 1986; Erickson, 1993, 2005; Zapf, Vogt, Seifert, Mertini, \& Isic, 1999). The origin of the concept stems from Hochschild (2003) who defined it as aspects of emotional management that take place in the private sphere to develop and maintain relationships (Hochschild, 2003; Zapf et al., 1999). Several researchers have since developed the concept further by applying it to aspects of social life which fall outside of work. Erickson (1993, 2005) used emotion work to explore marital roles and familial relationships. In her work emotion work signified:

'activities that are concerned with the enhancement of others' emotional well-being and with the provision of emotional support' (Erickson, 2005, p. 338). 
A similar definition has also been developed by England and Farkas (1986) who described emotion work as attempts made to empathise with others and their personal situations; Rafaeli and Sutton (1987) recognise this as a key aspect of emotional displays in the workplace for some occupations. Strazdins (2000) considers emotion work to be the actions undertaken to 'improve emotional wellbeing in others and to create cooperative and positive social relationships' (p. 41).

As a theory, emotion work provides a means of examining affective aspects of behaviour and interaction which aims to enhance the wellbeing of others through the creation and maintenance of relationships. Vassilev et al. (2013) study of social networks draws on the concept of emotional work in studying the personal networks of chronically ill individuals to examine illness management practices. Specifically, the authors were interested in exploring the individuals who comprised the personal communities (social networks) of chronically ill individuals; the circumstances that led them to become involved in providing support to these individuals; and the type of work they undertake to do this. One of the types of work explored was 'emotional work' which captured supportive actions undertaken to comfort worried or anxious individuals through everyday matters that commonly accompany long term illness management (Vassilev et al., 2013). The study found that having a broad set of relationships and diverse networks (beyond close family and partners) played a pivotal role in providing emotional support to chronically ill individuals.

Zapf et al. (1999) has criticised emotion work theory, arguing that there is a lack of consensus about how emotion work should be conceptualised and examined. Brook (2009) suggests that this lack of definitional clarity means that emotion work or emotional labour can be used as blanket terms, but that both oversimplify what can be a complex variety of social or affective actions.

\section{Emotional Labour}

A second social theory of emotions, emotional labour, stems again from the work of Hochschild (2003). Hochschild's more prolific and widely used notion of emotional labour refers to

'the management of feeling to create a publicly observable facial and bodily display; emotional labour is sold for a wage and therefore has an exchange value' (Hochschild, 2003, p. 7).

Essentially, individuals are required to internalise organisational expectations about how individuals should feel and act (feeling rules) and behave accordingly. This involves two different processes; surface acting (conforming to organisational 
expectations by presenting certain expected expressive gestures to an audience (Pisaniello, Winefield, \& Delfabbro, 2012); and deep acting (the use of emotion memory and association to align feelings and emotions with the feeling rules to create a desired emotional response) (Hülsheger \& Schewe, 2011). This theory has been applied to several research areas including service work (Hochschild, 2003; Wharton, 1993), nursing and care home labour (Lopez, 2006; Theodosius, 2008).

The theory has been criticised because, as Bolton (2005) notes, the definition of emotional labour developed by Hochschild (2003) is too deterministic and overlooks opportunities for independence of thought, action and vitality that individuals bring to organisations (Bolton, 2005; Brook, 2009). In other words, emotional labour theory assumes that individuals are passive and conform to organisational expectations, leaving no room for agency or honest social interactions in the occupational sphere.

\section{Emotional Capital}

The final social theory of emotion is emotional capital, which develops Bourdieu's notion of cultural capital and has been defined by Nowotny as:

'knowledge, contacts and relations as well as access to emotionally valued skills and assets, which hold within any social network characterised at least partly by affective ties' (Nowotny, 1981, p. 148).

This definition appears to be an oversimplification of what emotional resources are and creates an amalgamation of different forms of capital (human and social capital). A more precise definition of emotional capital is posited by Gendron (2004) who conceptualises it as the sum of emotional competencies that individuals develop through their lived experiences which can be used to guide decisions and for personal, cognitive social or economic development. Other researchers have likened emotional capital to a bank account where individuals could accumulate relationship wealth or a set of positive shared experiences which resources that can be drawn upon to ensure emotional success (Feeney \& Lemay Jr, 2012).

While Feeney \& Lemay Jr's (2012) discussion offers a more precise examination of the emotional resources that can be drawn upon by individuals to facilitate certain actions, it does not explicitly link emotional competencies to the management of social relationships. Furthermore, both iterations of this theory draw on Bourdieu's work by inferring that this form of capital can be used to advance one's social position by guiding decisions and behaviour. However, it does not appear to acknowledge more altruistic uses of emotional skills. For instance, nurses and care workers may 
use certain emotional skills to help support their patients and not necessarily to advance their own position or advancement in the occupational sphere. Also, both definitions remove or fail to acknowledge the social aspect of how emotions are used to create meaningful relationships. Instead, like with other forms of capital, emotional labour is viewed largely as a marketable asset.

\section{Conclusions from the Literature Review}

\section{Justification of choice of social theories}

The literature review has helped to inform a conceptual framework and research questions outlined in the following sections of this chapter, as well as shaping the mixed-methods approach taken in the study (elaborated in Chapters Three and Four) and the theoretical perspectives which will be used to interpret findings.

Social and human capital theories: social capital theory offers a way of examining how the Caseworker workforce operates to achieve their organisational aims and outcomes. This is particularly relevant in the current social and policy context in which partnership working has been heavily emphasised by the Welsh Government.

While there are many iterations and definitions of social capital theory this thesis uses both classical and neo-capital theorists by considering social capital to be an individual level resource that reflects individual investments which can be drawn upon to facilitate action (Lin, 1999). The thesis focuses on the individuals who comprise the Caseworker workforce and the relationships they build, manage and utilise to access resources deliver services to clients. If social capital was examined at an organisational level the diversity of expertise, skills and professional relationships that individuals bring to the Caseworker role would be glossed over. Social capital theory therefore offers a relevant interpretive framework of findings about how Caseworkers connect, manage and use their relationships and any associated resources to deliver services to older people.

Second, the thesis borrows from classical versions of social capital theory, specifically Putnam and Coleman's versions because they incorporate aspects of trust. Trust is an important aspect of social capital because it is through relationships that resources can be shared and accessed but '...trust is the glue that holds them [the relationships or networks] together' (Stephenson, 2005, p. 248). Consequently, trust could be an important explanatory dimension in helping to explain how Caseworkers are able to develop their networks of relationships and work effectively with their clients. 
Although this could be captured quantitatively, this aspect will be explored qualitatively to discern if and how trust has role in aiding the Caseworkers deliver services.

Finally, social capital requires an investment of time and energy by individuals to both build and maintain social networks of relationships in which social capital exists (Putnam, 2000; Putnam et al., 1994). Therefore, a Caseworker's age, past work experience and the length of time that they have been in post may be linked to the size of their professional networks and the amount of resources they can access to facilitate service delivery. These factors will be considered as part of the thesis' social network analysis.

The thesis will also draw on the theory of human capital, interpreting it as an individual level asset which includes the specialised experiences, skills and knowledge acquired over time through an investment in training, education and work experience (Coleman, 1988). In line with Bowles et al. (2001) and Glaeser et al. (2002), the thesis considers social skills and charisma as forms of human capital. This is because while these skills can help individuals to develop maintain their social capital; they are not in themselves social capital. Rather, they represent elements of human capital which can be enhanced through time and experience. So, while social and human capitals remain separate entities, they can overlap and aid in the development of each other. This is difficult to capture quantitatively and will therefore be explored qualitatively.

In summary, based on a critical review of social capital theory this thesis adopts a definition of social capital as the capital of relationships and the resources contained within them (Bourdieu, 1973; Coleman, 1988; Field, 2003; Putnam, 2000). Human capital is taken to represent the knowledge, skills (practical and emotional) and experiences that individuals accrue, and organisations invest in to generate a positive outcome e.g. increase social capital.

Network theory: this will be used to interpret the empirical findings of how Caseworkers connect with each other. Using network theory provides a useful and innovative way of examining the networking that occurs between Caseworkers.

Social network analysis: initial consultation processes which occurred prior to the commencement of the thesis led to the identification of a research topic which was of strategic importance to Care \& Repair Cymru. The literature review then showed that there was a gap in knowledge about this topic in relation to Caseworkers and 
the role they have in promoting independent living by facilitating ageing in place. A second outcome of these early consultations was the identification of social network analysis as a useful tool in exploring the professional networks that Caseworkers develop and utilise to deliver services to their clients.

After a critical review of the literature, it was decided that using an ego-centric ${ }^{8}$ social network analysis approach could provide a visual representation of the networking taking place between Caseworkers.

In contrast to traditional network analysis approaches in which the unit of analysis is the relationships that are embedded within the networks, the thesis will also examine the geographic context in which the networks are based. Some of the applied social network analysis studies reviewed (Coleman et al., 1966; Van den Bulte \& Lilien, 2001; West et al., 1999) have indicated that ignoring the context only provides a partial understanding of how individuals form relationships with others. These studies, along with the findings from the Auditor General for Wales (2018) report which found that the delivery and experience of home adaptation services varies regionally, have informed the research approach to include contextual factors in the analysis of how Caseworkers are networking with each other. The rationale underpinning this is that certain contextual or situational factors (e.g. geographic location) may how Caseworkers network.

Studies by Nicaise et al. (2012); Woodcraft (2011); Provan et al. (2005) and Coleman et al. (1966) provide a rationale for examining if individual Caseworkers are networking in such a way that they can be considered to be part of sub-groups within the larger Caseworker network. Analysing and empirically measuring how groups of individuals interact can identify individuals who occupy strategic position in the network in addition to illustrating if collaborative practices between agencies are occurring. This can provide an illustration of another way that Caseworkers are networking with each other and can also be used to make recommendations for service improvement or disseminating knowledge and training throughout the network.

Overall, both network theory and social network analysis will provide a means of examining how Caseworkers connect with their peers to deliver services and overcome structural barriers to facilitate independent living for older people.

${ }^{8}$ Approach social network analysis at the level of individuals to explore how they are connecting with others. 
Following on from this notion, examining individuals' capital can explain any differences in how individuals are connecting with each other in the Caseworker network.

Emotion work: finally, the thesis will use the concept of emotion work to understand and interpret the affective aspects associated with the Caseworkers' role and the nature of the interactions and relationships they develop with their clients. Despite its limitations, it was chosen over emotional capital and emotional labour for several reasons. Emotion work theory describes affective exchanges, actions and processes taken to develop positive and co-operative relationships which enhance the wellbeing of others without any real expectation of reciprocity (England \& Farkas, 1986; Erickson, 1993, 2005; Hochschild, 2003; Vassilev et al., 2013; Zapf et al., 1999). This is more relevant than emotion labour theory which encompasses acting and falsifying emotions to conform to organisational expectations; or emotional capital which views emotional competency and relationships as a form of exchange between people. Emotional labour theory implies that emotional engagement and the exchange that takes place in a workplace setting are highly controlled and inauthentic. Although Caseworkers are required to maintain a professional image while engaging with their clients, the notion of commercialising feelings and expression rules does not appear to translate to the realities of the job. This was observed during initial shadowing of the Caseworkers prior to data collection. In these instances, emotional engagement appeared to be genuine and go beyond feeling rules. Therefore, emotional labour does not adequately explain how Caseworkers are able to develop links with their clients and the affective actions they undertake to provide support.

Finally, considering that other theories of capital have been used as theoretical frameworks to interpret and analyse the networking activities of Caseworkers, emotional capital could have been a suitable theory for this thesis. While it does infer a connection with social capital and relationships, based on what was observed when shadowing the Caseworkers, this theory does not accurately represent the emotional processes used by Caseworkers to manage relationships with their clients. The key issue with this theory is that it assumes that relationships are equal or that each party can benefit from emotional exchange to some degree. Yet, from initial observations it appears that the relationships between Caseworkers are more complex and onesided. In other words, like other professional relationships Caseworkers invest in these relationships but do not derive any personal benefit from them. 
In sum, while it could be argued that emotion work appears similar to emotion capital theory, the key differences between the two are the themes of altruism and emotional wellbeing. Emotion work was therefore deemed a more appropriate interpretive framework because it focuses on the management of relationships between to promote well-being, rather than abstracting emotions to resource that can be used to generate returns.

From the literature reviewed, it appears that emotion work theory has the potential to explain the interpersonal aspects of the Caseworker role, specifically, how Caseworkers build and maintain links with their clients. This is potentially an important aspect of the Caseworker role because they may be fulfilling a role traditionally undertaken by the family, but due to recent changes to family roles and expectations others are needed to assume this responsibility (sees Chapter One).

\section{Conceptualising the Caseworker role}

Drawing upon the conceptual and theoretical premises of social network analysis and social capital literature, the Caseworker role will be explored in terms of the idea of a link agent who 'brokers' or facilitates relationships and connections. Tichy et al. (1979) describe 'links' in social networks as direct associations between pairs of individuals and in this thesis, encompasses the relationships between Caseworkers, clients and external organisations. Second, 'brokerage' type roles have been discussed in social network analysis and social capital literature. Burt (1997) posited that social structures are comprised of groups of people who may be unconnected; by inference, this would give certain individuals the opportunity to act as 'brokers', or 'a go-between or a bridge, linking two other actors that are otherwise not themselves linked' (Long, Cunningham, Carswell, \& Braithwaite, 2013, p. 339). Diani (2003) defines brokers in the context of social networks as:

‘.. an actor connecting other actors which are not directly related to each other...[their]most crucial property lies in their capacity to connect actors who are not communicating because of some specific political or social barrier, rather than the mere absence of practical opportunities' (pp. 107-108). ${ }^{9}$

Meyer (2010) in his discussion of connecting research and different audiences define brokers as entities that link otherwise unconnected actors which is crucial for ensuring that information and resources flow across individual and organisational boundaries. The notion of brokerage translates to the types of work activities that Caseworkers are expected to undertake as part of their job description ${ }^{10}$. Like brokers

${ }^{9}$ See also (Fleming \& Waguespack, 2007)

${ }_{10}$ The job description requires 'Co-ordination and implementation of packages of help for the Clients from different sources, e.g. Occupational Therapy, Social Services, Grants Department, Health, 
in social networks, the Caseworker facilitates the transfer of information between individuals or groups separated by a gap or barrier. Furthermore, as outlined in Chapter One, the nature of the Caseworker role requires them to operate at an intersection between housing, health and social care. Therefore, as brokers, Caseworkers have the potential to link individuals or organisations to each other and in doing so may be able to facilitate independent living through ageing in place.

Neo-capitalists do not appear to recognise any altruistic motivation in brokerage roles (Lin \& Chiou, 2011), but traditional network and capital theorists conceptualise brokers as individuals who service the needs of everyone by acting as go-betweens (Ward, House, \& Hamer, 2009). The traditional conceptualisation of brokers is relevant to the Caseworkers because there appears to be no advantage or gain for them to link clients with services.

Additionally, being a link agent also incorporates elements of case management. This is viewed as an effective way of facilitating and developing community based services (Bergen, 1992) and forms a key element of the Department of Health's approach to caring for individuals with long term health conditions (Department of Health, 2005; Ross, Curry, \& Goodwin, 2011). Research by Bergen (1992) examined case management in community nursing and identified three distinguishable aspects of the case manager's role; social entrepreneurship, service brokerage and an extended key worker or care co-ordinator role (Bergen, 1992). Furthermore, the applicability of case management in defining the Caseworker role as link agents is reinforced in a paper by Friedmann, Hendrickson, Gerstein, and Zhang (2004), which examined whether providing designated case management staff facilitates the delivery of comprehensive substance abuse treatment programs. In this article, case management professionals were understood to be individuals who work with clients to co-ordinate and facilitate service delivery of addiction treatment programmes (Friedmann et al., 2004). The type of work undertaken by the case managers in this paper is not dissimilar to the work that Caseworkers undertake. Therefore, like the case managers, Care \& Repair Cymru agency Caseworkers can also be conceptualised as both facilitators and co-ordinators of service delivery who link organisations and individuals together to enable the flow of information and services.

Charitable Organisations and specialist firms, progressing these in full consultation with the Client and other colleagues, both internal and external to the Agency.' 
In sum, this section has provided justification for conceptualising Caseworkers as link agents. This definition entails functions including brokerage, case management, coordination and facilitation of services. Understanding the Caseworkers in this way is highly relevant because as outlined in Chapter One, Care \& Repair Cymru Caseworkers work across health, housing and social care sectors. Therefore, it follows that Caseworkers would link with others to deliver services. Furthermore, linking and co-ordinating services reflects a broader organisational ethos of rationalising resources to make the best use of frontline services, which has partly arisen from the structural changes that Care \& Repair Cymru and their local agencies have recently gone through.

The conceptualisation of Caseworkers as link agents will be revisited and discussed against the backdrop of empirical findings.

\section{Research questions}

The literature review on social network analysis, social and human capital and emotion work theories has informed the development of questions which help address the research aims. It has demonstrated that different networking roles have important consequences for the structure and dynamics of networks of relationships (Nicaise et al., 2012; Stephenson, 2005; Woodcraft, 2011). Therefore, examining different patterns or ways of networking can explain how individuals act to make a network function.

Reviewing the capital literature has pointed to connections between networks and human and social capital (Ennis \& West, 2013), so exploring Caseworkers' capital may give an insight into how a network operates. However, the context in which the Caseworkers are operating should also be examined because previous studies (Coleman et al., 1957, 1966; Van den Bulte \& Lilien, 2001; West et al., 1999) suggest that there may be important contextual factors which can influence networking and the way individuals link with each other.

Finally, examining the perspectives of older people themselves may help determine whether their experiences of Caseworker interventions effectively involves the affective elements identified by others researching nursing (Bolton, 2000, 2001; Bone, 2002; Gray, 2009) and care work sectors (Aronson \& Neysmith, 1996; Bergen, 1992; Cancian, 2000; Lopez, 2006). 
In light of the literature review, the following research questions were formulated to address the research aims.

1.

a) What types of network connections are the Caseworkers building between one another within and across agencies?

b) Are there any subgroups in this Caseworker network?

2 .

a) Are there any distinct patterns of interaction (networking profiles) which capture how Caseworkers are connecting with one another?

b) What individual and contextual factors help explain these patterns of connectivity across the Caseworker network?

3.

a) What types of organisations outside of Care \& Repair Cymru do Caseworkers connect with across Wales?

b) What individual and contextual factors help explain the different types of external organisational links that Caseworkers build?

4. What can older clients tell us about their experiences of receiving support from Caseworkers?

\section{Chapter Summary}

This chapter has discussed the practicalities the narrative approach used to reviewing the literature. Then, a critical overview of social and human capital theory, network theory and emotion work was given. These theories were chosen because they informed the research questions, approach and design of this thesis.

First, theories of social and human capital were explored and defined. For this thesis social capital is understood as an individual resource embedded in networks of relationships. The resources are other individuals who each have an amount of human capital, which can facilitate actions or achieve outcomes. Therefore, social and human capital will be used to represent the sum of skills, knowledge, experiences and professional connections that Caseworkers bring to their roles.

Second, an examination of social network analysis and network theory literature has shown that these are useful theoretical approaches to examine the intraorganisational network of Care \& Repair Cymru Caseworkers because it can provide an illustration of which CWs connect the most with others and occupy important or pivotal position within the network. 
Finally, emotion work theory was examined as this theory offers an insightful way to analyse and examine the affective skills and personality traits that can be used by Caseworkers to develop effective working partnerships and help to deliver services to their clients.

This chapter concluded by offering a preliminary conceptualisation of the Caseworker role drawing from the Caseworkers' job description, two SIP projects, social network analysis and community practice literature. Drawing from these sources the Caseworkers can be conceptualised as link agents because of their position of linking with colleagues, external services and clients to deliver services and facilitate independent living for older people in Wales. This preliminary conceptualisation of Caseworkers will be confirmed and developed upon using the empirical evidence gathered in this thesis.

Finally, the research questions that this thesis aimed to address were outlined. These questions were developed from a critical review of the literature and considering the policy context of Wales which was established in Chapter one. Now that the pertinent literature which underpins this thesis has been defined and examined. The next chapter will explore the ontological and epistemological approaches, theoretical underpinnings and ethical considerations that have shaped the approach and design of this thesis. 


\section{CHAPTER THREE}

\section{Ontological and Epistemological Foundations of the Thesis}

This chapter presents the ontological and epistemological foundations that have influenced the design and approach that this research has adopted. The overall aim as suggested by Bryman (2008) is to link the epistemological position with the research and choice of methods. First, the emergence of pragmatism as a research paradigm and its relevance to this thesis is outlined. Then the rationale behind using a mixed methods approach for this thesis is discussed. However, the mixed methods research design will be discussed in Chapter Four. This chapter outlines the advantages and disadvantages of self-completion questionnaires and semi-structured interview schedules delivered through individual face-to-face interviews and focus groups as data collection methods, along with the reasons why these were chosen for this thesis. The final section of this chapter outlines the ethical considerations that this thesis had to address.

\section{Pragmatism}

This thesis has been influenced by a pragmatist paradigm, and in turn, a mixed methods approach. According to Johnson and Onwuegbuzie (2004) pragmatism considers knowledge to be both 'constructed and based on the reality of the world we experience and live in' (p. 18). Therefore knowledge, truth and meanings, are constantly changing and adapting over time. Researchers who adopt a pragmatist paradigm take a value orientated approach to research and believe that social research cannot be conducted with complete objectivity. This is because the subject of research are humans and the social world in which a researcher is embedded, and therefore retaining objectivity is considered to be almost impossible (Johnson \& Onwuegbuzie, 2004).

Pragmatism emerged as an epistemological position as the result of "conflicts" between two opposing ontological and epistemological perspectives: objectivism and constructionism. Ontology is our understanding of the nature of reality (Sarantakos, 2013), and guides the social research process by informing the other stages as to what the nature of reality is and what should be studied (Sarantakos, 2013). Whereas, epistemology is defined as the 'study of knowledge' (Benton \& Craib, 2011), or what is considered to be knowledge (Sarantakos, 2005). 
There are two dominant ontologies underpinning social research; objectivism that views reality as objective and external to individuals, and constructionism which sees reality as subjective and constructed by individuals. Objectivism is the ontological position which explains that 'social phenomena and their meanings have an existence that is independent of social actors' (Bryman, 2008, pp. 18-19). In other words, an autonomous reality exists with a distinct set of patterns and regularities that are independent of researchers or social actors (Bryman, 2008; Moses \& Knutsen, 2012; Sarantakos, 2005). This ontological position influences the epistemological considerations that underpin the quantitative approach to research.

In contrast, the ontological position of constructionism is based on the premise that 'social phenomena and their meanings are continually being accomplished by social actors' (Bryman, 2008, p. 19). In other words, constructionism acknowledges the integral role that individuals play in creating the 'patterns', categories or social phenomena that researchers are interested in studying (Moses \& Knutsen, 2012). As such, it is possible for individuals to observe the same phenomena but understand it in very different ways; hence, any attempt to understand the world in generalizable terms is inherently difficult (Moses \& Knutsen, 2012). Thus, research presents a 'specific version of social reality, rather than one that can be regarded as definitive' (Bryman, 2008, p. 19). The role of human agency means that constructionists believe that observational declarations are value-laden with inherent biases (Moses \& Knutsen, 2012), and therefore cannot be claimed to be neutral or consistent across researchers.

Unlike objectivists, researchers who adopt a constructionist position theorize that individuals do not simply experience the world in a direct and objective manner. Instead, it is perceived or interpreted based on individual (e.g. age and gender) or social characteristics (e.g. language and culture). Bryman (2008) summarises this by stating that social phenomena and categories are not only produced through social interaction but... they are in a constant state of revision' (pp. 19-20).

In sum, the broad differences between objectivism and constructionism stems from how each position views the nature of reality and what social research should focus on. For objectivists, reality is viewed as autonomous and existing externally to individuals. This means that research aims to measure aspects of social reality and use findings to confirm generalizable truths which represent one true reality (Moses $\&$ Knutsen, 2012). Constructionism on the other hand, views reality as subjective and constructed by individuals, therefore, social research aims to explore social phenomena from the subjective perspectives of individuals (Ibid). 


\section{From Ontology to Epistemology}

Although Crotty (1998) notes that ontological and epistemological issues often become intertwined because 'to talk of the construction of meaning is to talk of the construction of a meaningful reality' (p. 10)., they are distinct concepts and ontologies shape one's epistemology.

Constructionist ontology is frequently associated with interpretivism which involves researchers examining the 'subjective meaning of social action' (Bryman, 2008, p. 694). Moses and Knutsen (2012) state that the focus of the interpretivist epistemology fits into the constructionist ontology because value is placed on understanding social phenomena, despite there being more than one way to understand and interpret it. Interpretivism is associated with qualitative approaches which focus on capturing the subjective meanings behind phenomena and social action through observation and interaction and exploring opinions, views and perspectives around a particular social phenomenon (Bryman, 2008).

An objectivist ontology in contrast, is commonly associated with a positivist epistemological position, and with quantitative social research. According to Bryman (2008), positivists believe that the social sciences can and should apply the same methods as those used in the natural sciences, and that there is a reality which can be examined and measured objectively. Positivism also entails several assumptions that underpin its understanding of what knowledge is. One assumption, in keeping with an objectivist ontology, is that reality is external to individuals. Therefore, only social phenomena and knowledge claims that can be confirmed by the senses or through the application of scientific methods are genuine. A second assumption is that, scientific methods are value-free (Bryman, 2008) and any knowledge generated are regarded as social facts because they are objective, free of bias and '... in principle, theoretically independent' (Moses \& Knutsen, 2012, p. 8). Social facts can be tested (using scientific or quantitative methods) and are proven to be true or false (Benton \& Craib, 2011). Hence 'knowledge is arrived at through the gathering of facts that provide the basis of laws' (Bryman, 2008, p. 13). This links into the third assumption that research should use theory to produce hypotheses which can be tested and used to make generalisations (deductive approach to social research) (Ibid).

To summarise, the main difference between positivism and interpretivism is that positivism places emphasis on nomothetic knowledge (making factual statements that apply on a grand scale). Interpretivism, on the other hand embraces the knowledge 
gained by idiographic study in its own right, not just as a part of a larger study aimed at producing grand social facts (Moses \& Knutsen, 2012).

\section{Emergence of pragmatism}

As a paradigm, pragmatism has evolved in response to what Tashakkori and Teddlie (1998) have called the 'paradigm wars' (p. 3). This was a debate amongst academics in the social and behavioural sciences about which of the dominant epistemological models of positivism and interpretivism outlined above were superior to the other. A result of this debate caused many academics to adopt a purist and exclusionary stance towards epistemologies which has created a schism between qualitative and quantitative researchers (Ibid). This has led to the emergence of the 'incompatibility thesis' (Howe, 1988; Tashakkori \& Teddlie, 1998), which posits that quantitative and qualitative methods are theoretically incompatible because they are part of separate research paradigms entailing 'incompatible epistemological principles' (Bryman, 2008, p. 606). On the other hand, there is a contrasting argument ('compatibility thesis') that views quantitative and qualitative methods as compatible with one another. This position has been given the label of 'pragmatism' or 'paradigm relativism' (Tashakkori $\&$ Teddlie, 1998, p. 5), which is a third research paradigm.

In a pragmatist paradigm, knowledge, truth and meaning are viewed as being both a subjective construction and based objectively on the social world in which we are immersed (Johnson \& Onwuegbuzie, 2004). Creswell (2009) notes that the aim of pragmatism has been to find the middle ground between distinct philosophies or epistemologies and to find a workable solution to examining and solving social problems, phenomena and research questions.

\section{Mixed methods}

The thesis adopted a sequential mixed methods approach and aligns with a pragmatist paradigm (Johnson, Onwuegbuzie, \& Turner, 2007), because it acknowledges the virtues of constructionist and positivist paradigms together.

Johnson and Onwuegbuzie (2004) suggest that mixed methods research should 'use a method and philosophy that attempt to fit together the insights provided by qualitative and quantitative research into a workable solution' (p. 16). Creswell (2009) notes that this does not simply entail collecting and analysing both forms of data in a single study, but instead requires incorporating both approaches, philosophical assumptions and language into the research process. By bringing the two approaches together in this way, the strength of the study is greater than either approach on its own. 
A mixed methods research approach can entail using a combination of methods for different purposes. For instance, Denzin (1978) and Sarantakos (2013) refer to intermethod triangulation which combines research methods across different methodologies. The point of this is that the combination of methods reflects different ontological and epistemological positions which are brought together to examine phenomena. Holloway and Wheeler (2002) also talk of methodological triangulation or the practice of confirming or exploring findings generated from the use of one method with another method (Holloway \& Wheeler, 2002). These two processes can be reflected in a 'sequential mixed methods' strategy which involves 'conducting a study that mixes qualitative and quantitative research within one or more of, or across the stages of the research process' (Leech \& Onwuegbuzie, 2009, p. 271). Furthermore, Creswell (2009) notes that a sequential study design 'seeks to elaborate on or expand on the findings of one method with another method' (p. 14; see also Johnson et al., 2007), and Bryman (2008) notes that 'instrument development' commonly occurs in a sequential mixed methods strategy (pp. 609-619). Johnson et al. (2007) refer to this as sequential methodological triangulation, where the results of one stage of research informs the planning and implementation of the next method or stage of the research process.

The way in which the sequential mixed methods was developed and applied ${ }^{11}$ in this thesis is elaborated more in Chapter Four which focuses on research design. The following section explains how social network analysis works within a mixed methods approach.

\section{Mapping the reality of the Caseworker network: a social network approach}

Social network analysis is compatible with a mixed methods approach. Indeed, Edwards (2010) views a mixed methods approach as integral to social network analysis.

'A mixed-method approach enables researchers to both map and measure network properties and to explore issues relating to the construction, reproduction, variability and dynamics of network ties, and crucially in most cases, the meaning that ties have for those involved' (p. 6).

It is a way of objectively capturing the connections that Caseworkers build and explaining them through quantitative analysis. Social network analysis is therefore a set of methods that are used for 'collecting and analysing data from multiple individuals

${ }^{11}$ See Appendix item 1 for illustration of the ontological and epistemological underpinnings of this project. 
or organizations that may be interacting with one and another' (Provan et al., 2005, p. 605). It examines how individuals, organisations or other entities are connected (Ennis \& West, 2010), and is underpinned by the basic assumption that human beings are social animals who develop connections, relationships or 'social networks' through interactions with others within social and occupational spheres. It is these relationships in terms of the content and structural arrangement that are the unit of analysis in social network analysis (Ennis \& West, 2010; Provan et al., 2005; Rogers et al., 2014). The best way to capture the reality of the Caseworkers as part of a broader workforce is by visually mapping them through objective quantitative means. However, in recognising the interpretive limitations of this approach (see Chapter Eight); qualitative perspectives are also developed in the thesis.

\section{Methodological approaches to data collection}

'Methodology' means the study of which methods are most appropriate to measure or generate reliable knowledge and data (Moses \& Knutsen, 2012), and tends to be informed by the social theories used by a researcher. Essentially, the methodological approach informs the research design and links the choice and use of methods to the desired outcomes of a project. 'Methods' refer to the techniques or practical steps taken to gather and analyse data in order to answer a research question or test an hypothesis (Moses \& Knutsen, 2012).

This thesis used a combination of qualitative and quantitative methods to collect data, including:

- A self-completed, online questionnaire to collect quantitative data about the Caseworkers' personal characteristics and contextual factors, and their professional connections within and beyond their organisation; these allowed for social network analysis and further statistical analysis.

- Semi-structured interview schedules for qualitative data with Chief Officers and clients collected during face-to-face interviews.

- Semi-structured interview schedules for qualitative data with Technical Officers and Caseworkers collected through focus groups.

The way in which these were designed and used will be explained in Chapter Four. The next section of the chapter examines each method to outline the advantages and disadvantages and explain their relevance to the thesis. 


\section{Self-completion Questionnaires}

Questionnaires are one of the most commonly used social research tools and can be defined as:

'...methods of data collection in which information is gathered through oral or written questioning... Written questioning is accomplished through questionnaires' (Sarantakos, 2005, p. 239).

Questionnaires are similar to structured interviewing (or face-to-face survey methods) with the key difference that the researcher tends to be absent when questionnaires are completed, mainly if they are sent through the post or given directly to participants to complete in their own time (Bryman, 2008; Sarantakos, 2005). Despite the differences in the administration of questionnaires, they can all be classified as a 'self-administered or self-completion questionnaire' (Bryman, 2008, p. 216; Sarantakos, 2005, p. 239). Postal and web surveys are relatively quick and cheap to administer compared to other forms of data collection (Sarantakos, 2013), as there are little, or no travel costs compared to face-to-face survey methods (Bryman, 2008; Maxim, 1999).

Self-completion questionnaires are convenient for participants because there is no need for an interviewer to be present and it can be completed at their leisure (Sarantakos, 2013). Participants are also given the opportunity to consider the issues being explored which can lead to the collection of more thoughtful and pertinent data (Maxim, 1999; Sarantakos, 2013). In contrast, face-to-face survey methods have to be organised and arranged to suit people's schedules, and it would take longer to obtain the same number of responses (Bryman, 2008).

The absence of a researcher can be advantageous because the potential for interviewer effects or social desirability bias is reduced (Bryman, 2008; Maxim, 1999; Sarantakos, 2013). Additionally, questions are asked in a standardised manner, meaning that there is no risk of interviewer variability in self-completion questionnaires. As a consequence it is a stable, robust and uniform method of data collection (Sarantakos, 2013) which would be more difficult to achieve through faceto-face survey methods.

There are some limitations to self-completion questionnaires. The absence of a researcher can mean that participants cannot be prompted or probed for additional information, (Bryman, 2008); clarification about the meaning of questions cannot be obtained (Sarantakos, 2013); and additional data is difficult to collect once the questionnaire itself has closed (Ibid). Furthermore, during data collection the 
researcher cannot guarantee that the quality of the data collected remains consistent (Bryman, 2008), and the occurrence of missing data or partial responses (Sarantakos, 2013) is a disadvantage.

However, the most common problem associated with the use of self-completion questionnaires is the response rate (Maxim, 1999) as they do not provide opportunities for a researcher or interviewer to motivate participants to complete the questionnaire (Sarantakos, 2013). The lack of response rate can lead to bias because the representativeness of the sample can be questioned (Bryman, 2008).

The number and nature of the questions used can also entail problems. If a questionnaire has too many questions respondents can become bored and not answer questions in the most honest and truthful way. In a similar vein, Bryman (2008) noted that if questions were relatable to the research population then it is more likely that participants will complete the questionnaire. Therefore, if too many questions are unrelatable to the research population the response rate can be lower. Finally, with a self-completion questionnaire it is impossible to unequivocally determine and guarantee the identity of the respondent and the conditions under which it was completed (Sarantakos, 2013).

In sum, self-completion questionnaires were chosen over other survey methods because the evidence suggested that this method was most suited to collecting data from the Caseworkers within the timeframe of a $\mathrm{PhD}$ candidature. The ability to disseminate the questionnaire to the Caseworker population with minimal costs was the most efficient and effective ways to collect data.

\section{Semi-structured Interviewing: individual face-to-face interviews}

Interviewing is a type of oral questioning (Sarantakos, 1998) which can be used to generate 'subjective theory' (Flick, 2006, p. 155). This form of data collection is undertaken because those being interviewed have a form of specialised knowledge that the researcher wants to access and examine (Ibid). Interview data enables a greater understanding and exploration of the experiences, beliefs and emotions that are woven into the particular topic being examined, enhancing the validity of the data and findings (Coombes, Allen, \& Neale, 2008).

Interviewing can take many different forms depending on if an interview schedule is used, the types of questions employed, and whether individual or groups of people 
are interviewed at one time. The thesis used a semi-structured interview schedule containing a set of pre-set open-ended questions on topics that the researcher aimed to address (Jamshed, 2014) during individual face-to-face interviews. A semistructured approach places value on the perspectives and experiences of those being interviewed and gave participants the opportunity to speak in their own way (Byrne, 2004).

There are several advantages in using a semi-structured approach towards interviews. For instance, this type of interview guide provides a degree of flexibility in which answers are not limited to the interview schedule. Additionally, the use of open-ended questions can give participants the opportunity to elaborate and explore the parts of their answers that they consider to be important (Leidner, 1993).

Furthermore, a semi-structured approach limits the formality of the interviews allowing interactions to flow like ordinary conversations but remain purposive. This can have the effect of making the participants feel more at ease and more likely to disclose truthful information (Fielding \& Thomas, 2008). Participants may not have felt as comfortable disclosing such information, so freely if the interview had a more formal atmosphere. Finally, the flexibility of a semi-structured interview allows the questions to be adapted to suit the interviewees who may have different levels of competency and ability to articulate their answers, which can lower the possibility of non-response (Fielding \& Thomas, 2001).

Despite the advantages, there are some potential limitations and disadvantages of using a semi-structured interview approach. For example, there is the potential of non-response, which could stem from the meaning of the questions not being understood; a lack of knowledge; participants being reluctant to disclose information that could negatively impact them; and health issues (particularly with older people) may influence participant's ability to respond to questions. These factors could therefore lead to a degree of non-response bias within the interview data.

A disadvantage of this is that the presence of the interviewer could have resulted in interviewer bias, which could affect the validity of the data and findings that arise from interviews. However, the flexible design and approach of semi-structured interviewing reduces the possibility of this form of bias affecting the data and findings. 
There was also a risk that too much irrelevant data is gathered, which can make detailed analysis of the data problematic. However, this was ameliorated by administering the interview schedules face-to-face with participants (having a researcher present), meaning that interviewers can guide the conversation.

To summarise, this thesis employed individual face-to-face interviews which were guided by a semi-structured interview schedule. This method was suitable for this thesis because the evidence suggested that individual face-to-face interviews using a semi-structured interview guides were the best means of gathering knowledge of the local contexts in which Care \& Repair Cymru agency Caseworkers are operating in addition to exploring the experiences of older clients who made use of their services.

\section{Semi-structured interviewing: focus groups}

Two different semi structured interview guides were developed and delivered through focus groups. A focus group is a looser more flexible form of group interview that brings together a group of individuals to discuss, debate and address pertinent issues (Bryman, 2008). The focus group method comprises two aspects; the first is the group interview (as noted above). The second part is the focused interview where individuals are invited to participate because they have some level of involvement or expertise with the specific topic or issue (Merton, Fiske, \& Kendall, 1956). The discussion is led by the participant group, but it is guided by a moderator who also determines the topic of conversation (Bryman, 2008; Kitzinger, 1995; Morgan, 1996; Sarantakos, 2005). As noted above the interview schedule has pre-determined questions but allows a degree of flexibility for discussion.

Focus groups methods use group interactions or collective action is used as part of the method (Kitzinger, 1995; Webb \& Kevern, 2001). Group discussion, debate, exchange of experiences and perspectives is encouraged, giving this method a high level of validity because declarations can be stated, contradicted or reaffirmed by other participants (Webb \& Kevern, 2001).

There are other advantages associated with using focus groups as a research method. They do not discriminate against people who have difficulty expressing themselves in a written medium (Kitzinger, 1995). Even though this may not be much of an issue in the context of this research, there may be some participants who feel as though they can express themselves more eloquently and freely when responding to questions verbally. Focus groups can encourage participation from individuals who may feel intimidated by the notion of an individual interview, which can be perceived 
as too formal, leading to reticence to participate (Kitzinger, 1995). However, a group interview can create a more informal atmosphere where participants feel more comfortable to answer the questions posed by the researcher or moderator.

Recognised limitations to using focus groups include: participants hiding their true opinions for fear of backlash (Sarantakos, 2005); recording difficulties (Sarantakos, 2005); participants may talk simultaneously making it difficult to delineate between them (Kidd \& Parshall, 2000); group effects such as individuals dominating conversations, non-response and aligning with group expectations (Bryman, 2008); limited moderator balance between control over proceedings and conversational flow and freedom (Bryman, 2008); and difficulties in organising to accommodate all participants (Bryman, 2008; Sarantakos, 2013).

Despite these limitations, focus group methods do enable a researcher to examine not only what people think but how they construct meaning and think what they think (Bryman, 2008; Kitzinger, 1995). This is because the informal group interview can aid individuals to explore and refine their perspectives in ways that are more difficult to achieve through individual interviews e.g. group discussions and debates (Kitzinger, 1995). They also generate data on 'how the respondents themselves talk about the topics of the survey' (Morgan, 1996, p. 134), which can overcome the limitations of questions used in surveys, or questionnaires.

Given these advantages, a focus group approach was used to collect data about the Caseworkers, the context in which they are operating and the Technical Officer perspectives on this service and working with older people. The Caseworkers were also approached to participate in a focus group because it is a useful tool to examine and discuss survey data (Kitzinger, 1995). The focus group discussions gave the data more depth and validity because they could confirm, refute and explore the findings from the survey which can be considered to be a form of 'respondent validation' (Bryman, 2008, p. 377).

\section{Rationale for choosing pragmatism and a mixed methods design}

The rationale for using a mixed methods approach was informed by both a review of epistemology and ontology theory, and some initial interaction with Care \& Repair Cymru which occurred prior to the commencement of the fieldwork. There were opportunities to shadow some of the agency Caseworkers and speak informally to staff members. Additionally, a job description of the Caseworker role was also shared 
with the researcher because this would serve as a bench mark to explore and understand the Caseworkers.

After an initial examination of the job description and the informal observations gathered from the shadowing it became apparent that the Caseworker role was very multifaceted and highly complex. This was confirmed during interviews with Chief Officers where an affective dimension to the Caseworker role was alluded to, but this was not reflected in the job description. This showed that the reality of the Caseworker role was much more diverse and incorporated affective dimensions and suggested that a purely quantitative approach would not capture this diversity. While a quantitative approach captures and analyses the Caseworkers' networks and some of their characteristics in a systematic way; the qualitative methods would add more depth and exploration of the role within the local context in addition to the perceived outcomes of the Caseworker role from a diverse range of perspectives. Two openended questions were therefore included in the questionnaire to capture the perspectives of the Caseworkers. Using methodological triangulation in this way meant that the findings generated at each stage of the study could be enhanced or supported from several perspectives and in great depth.

Additionally, adhering to a pragmatic paradigm meant that knowledge of the Caseworkers' role is both objective and constructed. In other words, knowledge about this role exists but knowledge of the reality of the role is also constructed by coworkers, management, clients and Caseworkers themselves. In terms of gathering data, the job description formed an objective benchmark of what the Caseworkers' job entailed. However, it was also important to gather data from the Caseworkers' perspectives because this reflects the lived experiences or the reality of the Caseworkers' position. This data would add breadth to the exploration of the Caseworker role which could then be compared with the job description to enable a full understanding of what the reality of this role entails. Qualitative methods were utilised in recognition of the limitation of the self-completion questionnaires and the social network analysis. The data gathered from these methods would allow findings to be elaborated upon and contextualised (Bryman, 2008). For example, before the data collection phase an initial introductory group session with the Caseworkers took place to break the ice. As part of this session the Caseworkers were asked to list the links they make with external services and the reason for these links (which would be used to inform the design of the quantitative survey). This exercise captured part of the lived experiences of the Caseworker from their perspective using constructionist ontology. Information could have been gathered from senior 
management of local agencies or Care \& Repair Cymru, but this would not have been enough to capture the Caseworkers' reality from their perspective as there may have been instances where management were not fully aware of what Caseworkers do on the ground. The results from this session were translated into measures which could objectively measure the types of external connections that Caseworkers are making to deliver services to their clients (transformed into measures which fit into an objectivist ontology).

The social network analysis approach, whilst reflecting a positivist epistemology, allows this thesis the opportunity to confirm the idea that Caseworkers do link together, which has thus far not been substantiated. Social network analysis provides a means of visually representing the Caseworker network, which is useful because evidence from previous literature suggests that networking may not always follow a managerial hierarchy (Cross et al., 2002; Pow, Gayen, Elliott, \& Raeside, 2012). Therefore, social network analysis can illustrate how Caseworkers were connecting with each other, rather than what is assumed by others.

In sum, to fulfil the aims of this thesis, it is vital that a holistic examination of the Caseworker role is undertaken. To this end, pragmatism and mixed methods are appropriate because they offer a meaningful way to combine different forms of methods together within one research project in a way that is not epistemologically incoherent or incompatible (fitting with the compatibility thesis). Inter-method data triangulation provides a stronger research design and the best means to capture the complexity of the Caseworker role and provide depth about the reality and meaning of the Caseworker role. Simply, using a mono-methods approach to gather data or understand the Caseworker role would not be enough to capture and explore the complexity of the Caseworker role, their professional networks and the context in which they are operating in a holistic fashion.

\section{Ethical issues}

Several ethical issues had to be considered in the research design and data collection processes. This section will first examine these broad ethical issues before outlining how these concerns were addressed.

The first issue that had to be addressed was gaining permission to undertake the research. Care \& Repair Cymru were a part-funder of this research project, and their Caseworkers were the focus of this research. Prior to committing to the research project and bringing the PhD candidate on board, the head of agency support and 
development along with other members of senior management organised a meeting with all the Chief Officers to ensure that every agency agreed to support and be involved in the research project. Permission also had to be obtained from the ethics board in the College of Human and Health Sciences at Swansea University to ensure that the research design and aims met with the ethical standards and requirements.

\section{Vulnerability of participants}

Part of the research design (see Chapter Four) involved seeking the experiences and perspectives of older people. As a study population, older people may be vulnerable to illness, disability or frailty. The Welsh Government defines a vulnerable adult as:

'Someone who is 18 years of age or over and who is or may be in need of community care services by reason of mental or other disability, age or illness. They are or may be unable to take care of themselves, or unable to protect themselves against significant harm or serious exploitation' (Welsh Assembly Government, 2000, p. 14).

The definition above includes older persons who have support or care needs, and harm can take many forms including physical, psychological, financial, emotional, sexual abuse or neglect. Regarding the client study population, individuals were at risk of being emotionally vulnerable because the interview schedule contained questions asking about the reasons and issues underpinning their referral or relationship with their local Care \& Repair agency. This type of discussion could lead to embarrassment because the participants had to reminisce and relay experiences where they needed help for a personal problem or ailment. This had the potential to produce feelings of embarrassment or vulnerability because the participants were asked to share what could be private or personal information. Other ethical considerations were that as a study population, Caseworkers may have felt obliged to participate in the research, felt under undue pressure to talk about their work or felt that the process was unduly intrusive and would be inconvenient in terms of their workload (Social Research Association, 2013).

\section{Confidentiality, Anonymity and Informed Consent}

The confidentiality and anonymity of all the research participants was an ethical consideration which had to be addressed. Confidentiality is a particular concern when using and presenting the results arising from social network analysis (Zhang \& Zhang, 2009). Specifically the re-identification of participants can occur even if the personal identities of participants are excluded from publication (Zhang, Yu, Fan, \& Duan, 2013). To address this issue, data storage requires careful consideration. Any data collected and stored both electronically and on paper needs to be stored securely. This is to ensure that the identity of the participants remains anonymous and that 
their answers remained confidential in as much as only the researcher and members of the supervisory panel were able to access the data.

One of the biggest ethical concerns faced by social researchers is the issue of informed consent. This can be defined as a responsibility of a researcher or a research team to ensure that the aims, purposes and procedures associated with a research project is fully explained and understood. This means that before any individual participates they should fully comprehend the aims and objectives of the research project (Corti, Day, \& Backhouse, 2000; Wiles, Crow, Heath, \& Charles, 2008), what is required of them as participants, and how the data will be used and disseminated (Sarantakos, 2013). Participants must understand their rights when involved with the research project, including their right to withdraw at any point of the research process, without prejudice (Corti et al., 2000), and ability to refuse to answer any questions posed to them.

\section{Ethics Application}

To address these ethical issues the research was subject to scrutiny by Swansea University's ethics procedures before any fieldwork could be undertaken. This involved providing an overview of the research design, rationale, planned methods, samples of the interview schedules, information packs, consent forms and details of how ethical considerations would be addressed were submitted to ensure that the study complied with the Social Research Association's ethical guidelines and requirements (see Social Research Association, 2013).

In practical terms, to gain informed consent, the researcher developed and distributed 'information packs' to all the participants which detailed the purposes, procedures and aims of the research. For the clients, a follow-up telephone conversation was made by the researcher to confirm dates and times, and to answer any outstanding concerns. Both oral and written informed consent was sought from every study population.

Several measures were taken to protect potentially vulnerable participants. First, if the client participants were at risk of harm, then their identity could be revealed. This happened for one of the client interviews, where the participant was quite unwell (the interview data was later omitted from subsequent analyses). Second, prior to commencing the research, the researcher participated in Protection of Vulnerable Adults training, and was aware of the reporting process for POVA incidents. Additionally, a Disclosure and Barring Service certificate was applied and obtained 
before any field research was completed in line with Swansea University's ethical requirements. Finally, in line with the Mental Capacity Act (2005), to be able to give full informed consent to participate in the research a person must have the capacity or ability to make decisions, (NHS, 2018). Older people can be particularly vulnerable in cases of dementia, stroke or other illnesses which can impact cognitive ability. To address these concerns, certain exclusion criteria for participants were applied including ensuring that clients were capable of participating and giving informed consent (see Chapter Four). Additionally, while the consent forms were completed and delivered to the researcher prior to data collection, the researcher also discussed the consent forms with the clients face-to-face before the interview began to ensure that everything was fully understood and any questions the clients had were answered.

In terms of confidentiality and data storage, recorded interviews, focus groups, notes relating to this thesis and the personal details of participants including names and exact addresses were kept anonymous. Participants were given a reference number or code (known only to the researcher) and this was used in all documentation to ensure anonymity. Additionally, each Caseworker was given an identifier label which corresponded to their agency location, so the researcher could identify Caseworkers while maintaining their anonymity. Data (names, exact addresses, recordings, questionnaire data and transcriptions) were stored securely and all electronic data was password protected. The participant's real names and locations have been omitted from this thesis.

During conferences and presentations of the findings, anonymity was maintained. When presenting the social network data, the agency location in which the Caseworker operates was included in graphs and discussions, for instance $\mathrm{C} \& \mathrm{~V}$ represents a Caseworker from the Cardiff and the Vale agency. Aside from an agency location identifier, no other personal information was included and only the researcher would be able to identify which participants were represented by certain identifier labels.

Individual face-to-face interviews, Skype interviews and focus groups were voice recorded and reference numbers were used to identify and anonymise data which were then stored electronically on a password protected memory stick. Transcriptions and analysed data were stored securely, on a password protected hard drive and completed questionnaires were stored in a secure lockable cabinet that only the researcher was able to access. The same procedures were followed for the 
quantitative data. Consent forms and any files that refer to the coding of data (i.e. the pseudonyms used) were kept separate from the research data in a secure, lockable cabinet that only the researcher was able to access.

To offset any issues related to vulnerability for the researcher as a "lone worker" the researcher followed Swansea University's Centre of Innovative Ageing 'Personal safety guidelines for researchers and interviewers' and other training on issues associated with interviewing people in their own homes and safeguarding measures. On the ground, Care \& Repair Cymru agencies acted as a gatekeeper between the researcher and older clients. As part of this role, the Caseworkers identified individuals and home environments that would be suitable for interviews. In some instances, the Caseworkers drove the researcher to and from the client interviews. In addition to acting as a gatekeeper, this also safeguarded the researcher as these individuals were aware of where the interviews were being conducted and if required could be contacted if an unsafe situation arose. The researcher's mobile phone was charged and accessible during each interview. A designated individual (either a Care $\&$ Repair agency Caseworker or colleague within Swansea University) was informed of the time and likely duration of all interviews in addition to calling before entering and upon exiting a client's residence.

To alleviate any emotional distress, participants were given the opportunity to refrain from answering questions or withdrawing from the research process at any point, and this was reiterated at the outset of interviews and in a de-briefing document.

To ensure that Caseworkers did not feel pressurised or judged if they did not wish to participate, the researcher was introduced to, and liaised with various members of Care \& Repair Agencies (with Care \& Repair Cymru acting as gatekeeper) prior to the commencement of the fieldwork. Additionally, it was made clear to all Care \& Repair Cymru employees that the research was not a form of performance evaluation. Participants were also provided with an 'information pack' (see Appendix item 2) that outlined the importance of informed consent and their right to withdraw without prejudice at any stage of the research project.

Problems of professional intrusion were addressed by ensuring that times and dates of the data collection were convenient for Care \& Repair Cymru staff. For instance, the focus groups were coordinated to take place in a pre-arranged networking meeting organised by Care \& Repair Cymru to minimise inconvenience. Caseworkers 
were also given the opportunity to complete the online questionnaire at work. Client interviews were arranged by Care \& Repair agency Caseworkers on behalf of the researcher at times and dates that were convenient to the client participants, and interviews arranged to take place in their own homes to avoid travel costs.

\section{Chapter Summary}

This chapter has examined the ontological and epistemological foundations that have influenced the use of a mixed methods research approach guided by a pragmatic paradigm. This meant that the ontological and epistemological position of this thesis is that the social world is both independent and created by individuals. As such, knowledge is both objective and subjectively created and re-created by social actors. Therefore, social research should adopt whichever methods can best answer a research question or solve a problem. In this thesis, a sequential mixed methods approach (guided by pragmatism) was the most effective way to explore the Caseworkers' role by capturing the lived realities of Caseworkers themselves, exploring the reality of the Caseworker role as perceived by others, and comparing this to the Caseworker job description, which is an objective characterization of what the Caseworker role entails. Each method used in the data collection phase along with the advantages and disadvantages was conceptualised and discussed. Based on the evidence outlined in each section, the rationale underpinning the choice of each method was also outlined. Finally, the ethical issues that had to be considered and overcome in the design and implementation of the research methods were addressed. This chapter has discussed the rationales and epistemological frameworks which have underpinned the research design. This be explored in greater depth in Chapter Four along with the data collection and analytical approaches used. 


\section{CHAPTER FOUR}

\section{Research Design, Data Collection and Analytical Techniques}

This chapter presents the methods and analyses used to answer the research questions. The first section explores the overall research plan including the tools developed to gather the data. The second section of this chapter outlines the four study populations, recruitment frameworks and strategies used to recruit the study populations, and the inclusion and exclusion criteria. The chapter moves on to outline how the quantitative data from the online questionnaire was prepared for different kinds of analyses including what social network measures were captured, and how the capital and contextual variables were created. This section also describes the different kinds of quantitative analyses used to answer the research questions, including social network analysis and statistical tests such as ANOVAs, Spearman's Rho correlation tests, Chi-square tests of independence and Kruskal-Wallis tests. The final section outlines how the qualitative data generated from the client and Chief Officer interviews and the focus groups with Technical Officers and Caseworkers were prepared for analysis. Specifically, how the data was transcribed and the approach to undertaking the thematic analysis will be explained.

\section{Research design}

\section{Exploratory sequential mixed methods approach}

Drawing on the explanation given by Creswell (2014, pp. 225-226), an exploratory sequential mixed methods approach was adopted, which meant that data was collected at different times, and locations, and from different study populations. The fieldwork was completed over the period of September 2015 to September 2016. Four study populations were involved including Chief Officers from all agencies, and a selection of Technical Officers and Caseworkers from across all 15 agencies. The fourth group were Care \& Repair clients.

The first stage of the research design (see Figure 4.1- Stage 1) was exploratory and was undertaken to develop an understanding of each agency within their local context, in addition to identifying potential issues faced by agency Caseworkers. Qualitative data were gathered through individual face-to-face interviews with all Chief Officers and a focus group with a selection of Technical Officers. The Chief Officer interviews were focused on identifying issues at agency, regional and national levels, which they would be aware of, and how these might affect the work of each 
agency. The focus group was designed to provide insights into the sorts of activities agency Caseworkers undertake, how Technical Officers interact with them, and to explore the emotional aspects associated with frontline service delivery in this sector.

These findings were then used to inform the second stage of the research (see Figure 4.1), which involved the design and use of a self-completed online questionnaire that was sent to all Caseworkers employed by Care \& Repair. This data would be used to map and analyse how Caseworkers were connecting with each other using social network analysis techniques, the nature of the links they develop with other services and organisations beyond $\mathrm{C} \& \mathrm{RC}$, and to explore how individual and contextual factors might be associated with their networking patterns.

Figure 4.1- Stages of the exploratory sequential mixed methods research design

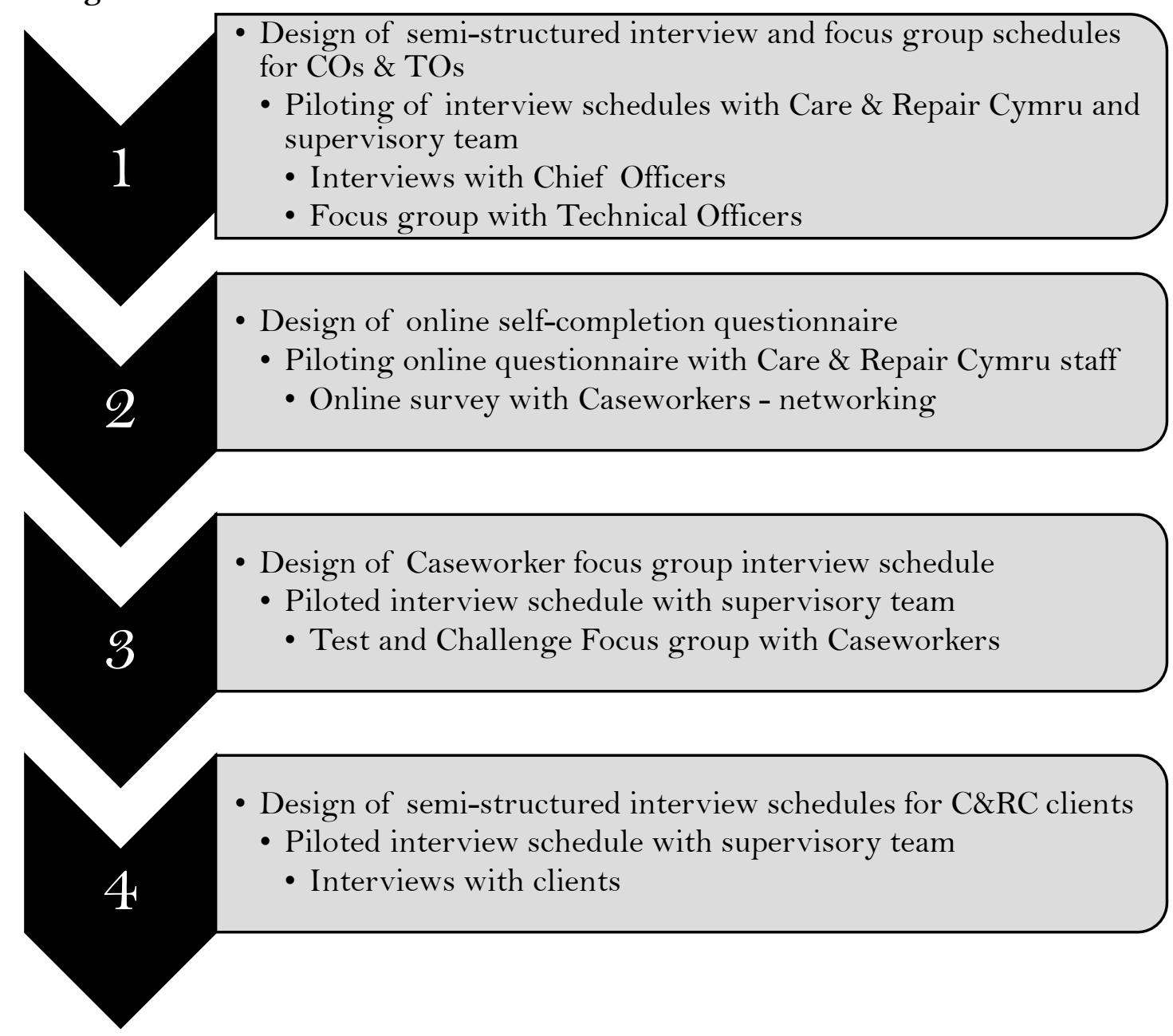

The Caseworker questionnaire also had sections that asked for more qualitative or open-ended responses and provided an opportunity for them to write about their experiences and thoughts regarding their work, including aspects they thought may be overlooked. The issues raised in these sections were explored during the test and challenge focus group in Stage 3. 
Stage 4 of the research involved qualitative interviews with older clients of C\&RC. It was intended that the data gathered would provide insights into clients' perceptions of Care \& Repair Cymru interventions.

\section{Design of data collection tools}

Data collection tools consisted of an online and printed version of a self-completion questionnaire for Caseworkers, two different semi-structured interview schedules for use during individual, face-to-face interviews with Chief Officers and clients; and two different semi-structured interview schedules for focus groups with Technical Officers and Caseworkers. The semi-structured interview schedules were piloted with members of the supervisory team to ensure that the questions were clear, suitable for the study population, and would promote discussion on desired topics. The online self-completion questionnaire was piloted with members of the supervisory team and staff from Care \& Repair Cymru.

\section{Online Caseworker questionnaire}

A survey tool (WebQuest) developed in-house at Swansea University was utilised to develop the questionnaire which included both closed and open ended questions to ensure that Caseworkers were given the opportunity to elaborate more in-depth answers if they wished (Bryman, 2008; Sarantakos, 2005). The questionnaire comprised of 26 questions divided into 5 sections (see Appendix item 3).

Section A included questions on Caseworkers' individual characteristics including agency location, age, level of education, vocational qualifications, training qualifications, past work experience and how their past experiences aid them in their role. Section B aimed to explore the different Caseworker roles and their responsibilities including job titles, funding, the length of time in post, working patterns, and main tasks. These questions capture the social and human capital of the Caseworkers (questions 2-13 see Appendix item 3).

Section C captured data for social network analysis. Caseworkers were asked to indicate other Caseworkers that they linked with for the purposes of work. The 15 agency locations were listed for this purpose so that to list Caseworker ties in particular geographic locations could be identified (See Appendix Item 3-Section C).

Questions in Section D were designed to capture the breadth of external service links and frequency of contact. This section was informed by a workshop which took place 
in January 2015 and asked participants to indicate which organisations they linked with for each of the sixteen categories identified from the workshop data and the Caseworker job description. Additionally, respondents were asked to indicate the frequency of contact with these external services or organisations, and if in their opinion, they thought that the service was good (see Appendix item 3). Section E aimed to explore wider contextual issues that can affect the Caseworkers' work (see Appendix item 3 section E, question 23) and included questions about difficulties they have experienced, improvements which could be made to improve the working environment and aspects of their role which they considered were not fully recognised.

\section{Semi-structured interview schedules for Chief Officers and clients}

Each semi-structured interview schedule was used for face-to-face interviews and comprised a list of open-ended questions and probes (Bryman, 2008; Fielding \& Thomas, 2001). The Chief Officer schedule (see Appendix item 4) covered topics about the areas and locations that the agencies covered, their client base (e.g. average age, gender, socio-economic background and service requirements), information about the Caseworker role and their responsibilities, patterns of working, how their role intersects with the Technical Officer role and the average number of visits made in a month. It also covered contextual issues relating to the agency (e.g. cross-sector or collaborative working; contextual issues that can impact service delivery) and an open-ended question to facilitate broader comments about the nature of the Caseworker role and the local contexts in which they worked.

The client schedule (see Appendix Item 5) which was used in Stage 4 of the research process included topics on reasons for contacting the service, the nature of the Caseworker intervention and outcomes (e.g. building works undertaken, welfare benefits applied for), and their thoughts about the service as a whole.

\section{Semi-structured interview schedules for Technical Officers and Caseworker}

Interview schedules developed for the focus groups were also semi-structured, making use of open-ended questions and probes. However, in the focus group setting debate and discussion was encouraged.

The Technical Officer focus group was designed to gain their insights into interactions with the Caseworker service. The interview schedule (see Appendix item 6) comprised broad questions about their role, the demand and types of clients they 
encounter, how their role interacts with the Caseworker service, challenges on the ground and an open-ended question to facilitate broader comments.

The Caseworker focus group interview schedule (see Appendix item 7) themes that had emerged from the answers to the open-ended questions in their online questionnaire and were designed to elicit more information about these themes and to ensure that the interpretation of their answers had been accurate. The schedule covered themes on the notion of support (as a means of teasing out emotion work themes), what 'going the extra mile' meant to them, issues around job role uncertainty and finally, the demands placed on the casework service in a context of welfare retrenchment.

\section{Study populations, sampling frames, recruitment and data collection}

Four study populations (Chief Officers, Technical Officers, Caseworkers and clients) were recruited using different sampling strategies. All research participants were provided with an 'information sheet' to provide basic information about the research; a 'recruitment letter' detailing why they have been approached to take part in the study; and an 'information pack' containing a consent form which explained what could be expected from participating in the research project (see Appendix Item 8). The consent forms were completed and returned to the researcher in the post or via email as a signed, scanned copy, or were collected prior to interview.

Chief Officers: an exhaustive sampling approach was adopted and Chief Officers from all agencies $^{12}$ across Wales were invited to participate in the fieldwork. They were approached by the gate-keeper (the head of agency support and development of Care \& Repair Cymru) to participate in individual face-to-face interviews. In terms of the recruitment process, following initial contact from the head of agency development and support, the Chief Officers were contacted individually. The response rate for this stage of the research process was $100 \%$, so 15 Chief Officers were recruited and participated in individual face-to-face qualitative semi-structured interviews.

Technical Officers: a convenience sampling approach was used to recruit Technical Officers based on their possible attendance at one of their regular networking meetings. This networking meeting in principle involved participation of every

\footnotetext{
12 At the time, this research was undertaken Care \& Repair were about to embark upon a major restructuring of their organisation. As a result, despite the existence of 22 regional agencies at the time, there were only 15 Chief Officers in post.
} 
agency across Wales and took place in a Mid-Wales agency office. Once the gatekeeper determined which Officers were planning to attend this meeting, a list of names was given to the researcher and all those listed were contacted by email. In total, 20 Technical Officers attended the meeting, and all were recruited and interviewed as part of the focus group.

Caseworkers: for purposes of completion of the self-completed online questionnaire, an exhaustive sampling approach was used for the Caseworker population. This meant that every Caseworker (approximately 78 in total) in each of the 22 local authorities in Wales were approached to complete the online questionnaire. This stage of data collection took place between February and September 2016. Participants were offered two methods of self-completion; online or paper. The online survey was contained in an external website, and once consent forms had been returned Caseworkers were sent another email containing an e-link to the website which contained the questionnaire or a hard copy of the questionnaire for the Caseworkers to complete. After the initial invitation email was sent to Caseworkers, 2 follow-up emails were sent out to non-responders to encourage a higher response rate. If Caseworkers had not responded after the three waves of invitation emails were sent, the Chief Officers of the agencies which had lower response rates were contacted to discuss potential solutions that could be put in place which would better enable the Caseworkers to complete the questionnaire.

Interim findings (comprising $54 \%$ or $n=42$ participants) were presented to Care $\&$ Repair Cymru after which the organisation issued another email to all Care \& Repair Caseworkers asking them to complete the questionnaire. Due to the low initial response rate, the questionnaire remained available online until the 05/09/2016. Finally, 55 Caseworkers (out of 78) were recruited and completed the online questionnaire; this meant that the survey had a response rate of $70.5 \%$. For reasons of confidentiality, the agency by agency response rate figures cannot be provided, so they have been grouped together under North (3 agencies), South (11 agencies) and Mid-Wales ( 1 agency). The non-response rate by region is: North Wales agencies $10.5 \%(n=2 / 19)$; Mid-Wales agency 40\% (n=2/5); and South Wales 35\% $(n=19 / 54)$.

Recruiting Caseworkers to the focus group was achieved using a convenience sampling approach. This was based on the attendance of Caseworkers at a networking event hosted by Care \& Repair Cymru. Care \& Repair Cymru supplied a list and work contact details of the Caseworkers who attended a networking event and provided a time slot during the day in which the focus groups could be conducted. The 
individuals on this list were informed via email of the aims and objectives of the focus group and were approached face-to-face on the day of the event to request participation. The sample comprised 27 Caseworkers (out of 78 ), which yields a response rate of $35.1 \%$.

Care \& Repair Clients: the clients were approached in part because the funders requested this to provide empirical evidence of the impact of Caseworkers. The other rationale underpinning the inclusion of Care \& Repair clients was to capture another perspective of the reality of the Caseworker role which may shed light on actions that occur which are not detected by the other study populations. Specifically, the affective dimension of role and how this links with service delivery.

Clients of Care \& Repair were identified using a purposive sampling approach. For pragmatic reasons (time/travelling constraints) the sampling frame was derived from the client lists in four agencies in South Wales: Swansea, Neath Port Talbot, Bridgend and Carmarthenshire, and participants were approached to participate in a face-to-face interview by Caseworkers from Care \& Repair agencies. Clients were selected using a 'purposive sampling strategy' (Bryman, 2008, pp. 415, 458; Robson, 2003, p. 264). Ten clients were approached to participate, however during one of the interviews, despite consenting to participate in the project it became apparent that the client was feeling ill. She had been recently discharged from hospital and was very confused and unable to answer a lot of the questions. This client was deemed to not have capacity to consent. In line with ethical requirements this interview was terminated, and the local Care \& Repair agency was contacted to inform them of what had happened. The interview data was also excluded from the analysis because the participant was unable to answer many of the questions.

Care \& Repair Cymru agency Caseworkers approached clients on my behalf to ask them to participate in an interview. There were some inclusion and exclusion criteria given to the agency Caseworkers, which had to be considered when identifying individuals to participate. For instance, clients had to be English speaking and have capacity to be able to fully consent to participate. In the first instance, the agency Caseworkers selected potential participants from their client lists and then contacted clients that they believed would be willing to participate via telephone. If the clients consented verbally during the initial telephone conversation, the gatekeepers sent them a 'client information sheet', a 'client recruitment letter' and a 'client information pack' (see Appendix item 8) containing a consent form. Some clients received these documents in the post, while others received them directly from their local 
Caseworkers who guided the clients through the forms during a face-to-face meeting. These documents gave more details about the research project and what would be required of the clients should they wish to participate.

Some of the consent forms were then returned to the researcher in the post (a stamped addressed envelope was included with the information pack); others were collected from the regional Care \& Repair agency office where the interviews were taking place. The Caseworkers also liaised with the consenting clients to arrange the time and dates of the interviews. However, a few days before the interviews were due to take place, the participants were contacted individually by the researcher over the telephone to confirm the time and dates of the interview and to answer any unresolved questions. Before commencing the interviews, the researcher read through the consent form with the participants one final time to ensure that participants understood their rights and were able to give full informed consent to participate. The final study population of Care \& Repair clients comprised 9 individuals who had made use of Care \& Repair services in the past, which gave a response rate of $90 \%$.

\section{Inclusion and Exclusion criteria}

For the three Care \& Repair study populations there was only one inclusion and exclusion criteria; the Caseworkers, agency managers and Technical Officers who were working for Care \& Repair agencies at the time of data collection would be approached (in an exhaustive sampling strategy). There were no exclusion criteria based on age, race, gender or working patterns (e.g. full or part time).

However, the inclusion and exclusion criteria for clients were a bit more nuanced. The inclusion criterion was based on geographic location. The qualitative face-toface interviews were conducted in four counties of South Wales, and each interview location was required to be commutable within one day. Client's also had to have the ability to provide full informed consent for participation (see Chapter Three). The final inclusion criterion was that the clients would be required to be able to understand and communicate verbally in English.

The inclusion criteria for the clients included clients being able to give full, informed consent (which was sought following the Mental Health Capacity Act 2005, see Chapter Three). For clients to be able to participate they must have been:

- Willing to participate;

- Able to give informed consent both oral and written, and in instances where 
informed consent cannot be obtained, the participant would have been excluded from the study;

- Able to understand and verbally communicate in English.

There were no exclusion criteria based on age (except that the clients had to be over the age of 65 to be logged in the $\mathrm{C} \& \mathrm{RC}$ client database), gender or ethnic background.

\section{Data collection: four stages}

Data was collected sequentially over four stages (See Figure 4.1). Stage 1 involved 15 interviews with Chief Officers and 1 focus group with 20 Technical Officers. The average length of the interview with the Chief Officers was 66.50 minutes; while the interviews ranged in duration from 24.27 minutes to 108.33 minutes. The focus group with Technical Officers comprised of 20 individuals along with the head of agency support and planning (facilitator and gate-keeper) and lasted 57.27 minutes The online self-completion questionnaire for Caseworkers was carried out for Stage 2 and 55 people participated.

Stage 3 involved the test and challenge focus group with Caseworkers. This was completed with 27 participants in addition to the head of business development who acted as a facilitator for the session. This focus group lasted 49.15 minutes. In Stage 4, 9 clients were interviewed from 4 different counties in South West Wales with the average client interview lasting 32 minutes (the shortest interview was 15.06 minutes while the longest interview lasted 49.15 minutes).

Focus groups and individual face-to-face interviews were recorded using two audio recording devices. The main reason underpinning this action was to ensure that the sessions were recorded and that no data was lost. Essentially, if one recording device failed, the other device could record the session. Additionally, using two recording devices in the focus groups meant that the researcher could accurately monitor which participants were speaking. In the Technical Officer focus group participants were asked to state their name and agency location before or after answering a question. This technique proved useful for initial transcriptions purposes, but any identifying text was later omitted from the transcription. The Caseworker focus group was run differently, as participants were split into groups and provided with worksheets which outlined the questions to be discussed. They were given a limited time to write down and debate their answers, before nominating a member to feedback answers to the moderator. This proved effective and ensured that recordings were clear. 


\section{Quantitative data preparation and analysis \\ Social Network Analysis}

Social networks can be analysed from the macro level (whole network) perspective to the micro-level (ego-centric) perspective. According to Provan et al. (2005), a macrolevel approach entails collecting data from every individual in a network using questionnaires or structured interviews. However, this thesis did not adopt a macro level network analysis, because not all of the individuals involved in the network of Care \& Repair Cymru agencies were approached. For instance, when examining the potential network actors that are present in Care \& Repair Cymru agencies there are many frontline workers who help to deliver services to older people including administrators, Technical Officers, Chief Officers, Occupational Therapists as well as local social enterprise schemes. But these individuals were not incorporated into the social network analysis aspect of this thesis.

Instead, an ego-centric or micro level approach was adopted where one group of individuals within a larger organisation are explored (DeJordy \& Halgin, 2008). This reflects the broader aim of the thesis of examining individual entities (Caseworkers) operating across different settings, their networks and the different types of links that they make (Ibid).

The reason for this approach was two-fold. The first was a practical consideration; due to the time restrictions in within which this study operated, it was not possible to gather data about all the different working groups in all the Care \& Repair agencies throughout Wales. The second rationale is that taking a micro-level network analysis approach to studying an organisation that operates within the health, housing and social care sectors, is that the results could 'provide understanding about how our microlevel relationships combine to create and/or challenge broader social structures' (Ennis \& West, 2010, p. 41).

The data generated from section $\mathrm{C}$, question 17 of the Caseworker questionnaire asked each participant to; 'please provide the names of any Caseworkers in any of the agencies that you have contact with for work purposes'. The data generated from this question was converted into a data matrix which can be used to undertake social network analysis (see Appendix item 9). In practical terms the preparation of the data involved: recoding the names of the Caseworkers and using, Microsoft Excel to create the data matrix that could be imported into UCINET and NetDraw for social network analysis purposes (see Figure 4.2 in Appendix item 9). 


\section{Measures used in SNA}

Individual measures of indegree and outdegree along with their normalised measures were calculated in UCINET along with the betweenness centrality measures. The results could then be transferred to NetDraw where the Caseworker network could be visualised and examined to determine how the Caseworkers were networking as individuals.

Betweenness centrality, degree centrality, indegree and outdegree were identified as being the most appropriate measures because they are individual level properties that capture how Caseworkers are connecting with each other ${ }^{13}$. Specifically, they can be used to identify if there are any particularly important individuals in the network and where they are located. Examining where individuals are located in social networks and how they connect provides a valuable insight into the dynamics of a network because

'a person's location within a social network can affect the volume, quality, and timeliness of information to which he/she has access, and how connections within a group can affect group cohesion, coordination, trust, knowledge sharing and problem solving/ innovation' (Meltzer et al., 2010, p. 2).

Centrality is an individual level property, that does not have a single definition, rather it is a collection of concepts (Borgatti, Everett, \& Johnson, 2013). Simply put, it is a quantitative measure of the 'contribution the node makes to the structure of the network' Borgatti et al. (2013, p. 164); in other words, centrality can refer to an individual's structural importance to the rest of the network (Ibid). Therefore, centrality can be conceptualised as 'falling under the general rubric of social capital concepts, in which a node's position is a source of opportunities and advantage' (Borgatti et al., 2013, p. 164).

\section{Degree centrality}

Degree centrality is the simplest centrality measure that can be used in social network analysis, and in basic terms this measure is a count of the number of ties or connections an individual has. This can be broken down into two components: the "outdegree score", which in this case refers to the number of colleagues that Caseworker reports linking with; and an 'indegree score', or the number of reported contacts received by one Caseworker from all others (Kruskal \& Snir, 1986). As illustrated in Figure 4.2. Combining these two measures gives the total number of connections an individual has (all-degree).

13 To respond to research question 1a) What types of network connections are the Caseworkers building between each other within the Care \& Repair organisation? 


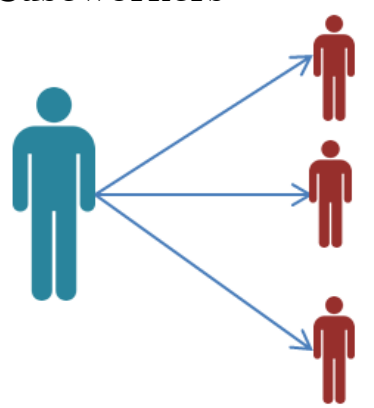

Example of outdegree of 3 connections

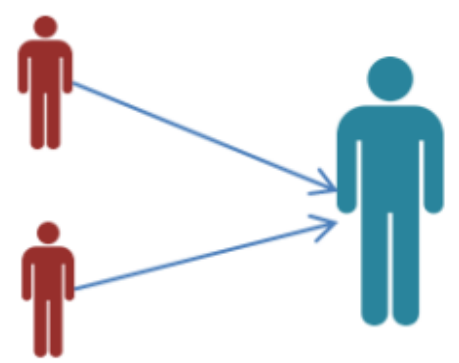

Example of indegree of 2 connections

Having high measures for both indegree and outdegree or all degree centrality suggests that there must be some reason why a Caseworker has a high score. There are several ways in which these scores can be interpreted. For instance, Borgatti et al. (2013) contend that outdegree could signify 'gregariousness or expansiveness' (p. 176), or an individual could be considered to be highly influential because they are able to reach more individuals within their network (Hanneman \& Riddle, 2005). Such individuals can be important in spreading innovation or knowledge throughout a network which can be important in the maintenance of an organisation.

On the other hand, a high indegree measure suggests that an individual has an amount of prestige which draws others into contacting that individual (Hanneman \& Riddle, 2005). From the perspective of social and human capital theorists (Adler \& Kwon, 2002; Becker, 1994; Burt, 1997; Lin, 1999) these individuals can be considered to be important because they have knowledge, skills or contacts that others wish to access or utilise (Borgatti et al., 2013; Cambridge Intelligence, 2017; Scott, 1991). Network theorists such as Borgatti et al. (2013) assert that the indegree denotes "prestige or popularity' of a person in a network (see also Hanneman \& Riddle, 2005).

These measures will inform and guide the interpretation of the results of the analyses because it raises the question as to why there may be different centrality measures. For instance, is there a characteristic that determines how individuals are networking i.e. do they have specialist knowledge that others wish to access which gives them a high indegree measure?

\section{Normalised measures of indegree and outdegree}

For the purposes of analysis, a normalised measure of degree centrality was utilised. This is a standardised degree centrality measure which makes the data more suitable for statistical analysis. This approach mirrors the practice of previous researchers who have used a normalised measure to compare the centrality between two different 
networks which may be of different sizes (Borgatti et al., 2013; Everett \& Borgatti, 2005).

\section{Betweenness Centrality}

As with degree centrality, betweenness centrality is also an individual level property which can be measured using social network analysis (Hanneman \& Riddle, 2005; Newman, 2005).

This measure focuses on measuring the extent to which an actor is connected to other nodes who are themselves not connected to each other. Essentially, this is a measure of the potential that an individual has to act as a bridge between other unconnected individuals (Hanneman \& Riddle, 2005; Newman, 2005; Wasserman \& Faust, 1994; Wey et al., 2008).

\section{Figure 4.3-Illustration of Betweenness Centrality measures for Caseworkers}

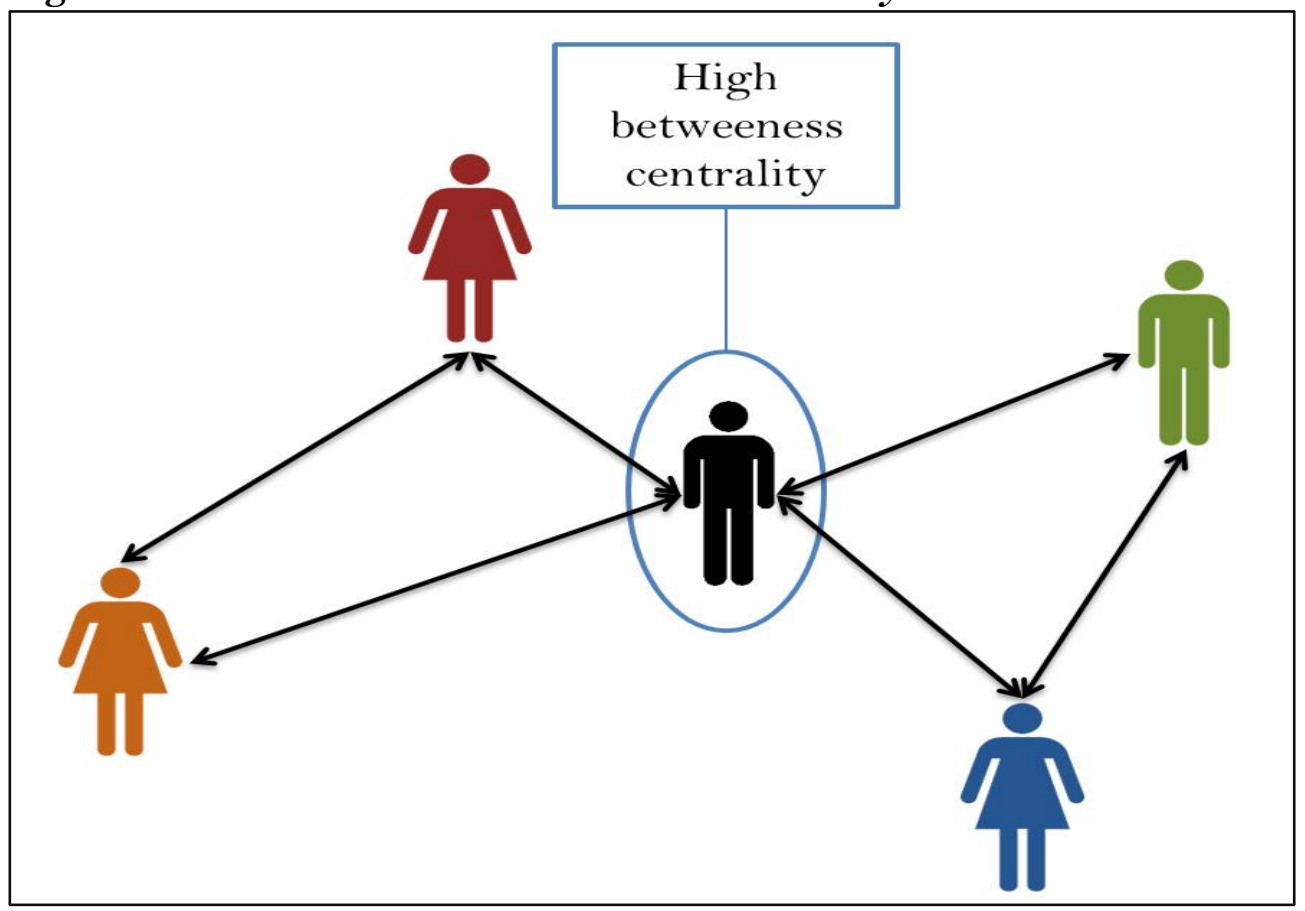

As indicated in Figure 4.3, an individual who has a high betweenness centrality measures acts as a bridge between unconnected individuals. One way to measure betweenness is to count the number of times an individual lies on the shortest possible paths between two otherwise unconnected individuals (Hanneman \& Riddle, 2005; Yang et al., 2017). This captures the potential for certain individuals to control the flow of 'information and resources exchanged between other pairs' (Yang et al., 2017, pp. 62-63) of actors. This provides another means of examining how Caseworkers are connecting with others in the network because individuals who do not have a high number of connections may occupy essential positions in the network and can 
facilitate the flow of knowledge and information between different parts of the network. In other words, an individual does not need to have a high degree centrality to have a high betweenness centrality measure. Also, unlike other centrality measures, betweenness is not contingent on the directions of the connections between individuals. Furthermore, due to the nature of this measure, betweenness centrality typically has high levels of variance.

\section{Normalisation of variables developed for SNA}

Caseworkers' individual indegree, outdegree and betweenness scores were computed using UCINET. Such measures are called absolute measures of centrality. Absolute measures cannot be used to compare the centralities of networks with different numbers of units. Therefore, these measures were normalised (in UCINET) to a range between 0 and 1 (see Appendix Item 10 for details of calculations).

\section{Clique analysis}

Another stage of social network analysis, clique analysis (or 'bottom up' Social Network Analysis approach) was used to examine how Caseworkers were linking with each other as groups.

A clique can be defined as a "subset of points in which every possible pair of points is directly connected by a line and the clique is not contained within any other clique' (Scott, 1991, p. 114). It is these cliques or subgroups which illustrate how the larger Caseworker network is composed of smaller ones. This analytical approach views the Caseworker network as a complex structure of relations which is built of small, simple relations i.e. dyads and triads which combine to create bigger but still closely connected substructures, sub-groups or cliques, which overlap and connect to create the larger complex network (Hanneman \& Riddle, 2005). Analyses of cliques are generally performed so that the sizes, variations and overlaps of cliques can be identified and explored (Hanneman \& Riddle, 2005). This enables an examination of which individuals comprise the sub-structures, where these cliques overlap (if at all), and which actors are the most central or the most isolated from the identified cliques.

UCINET's clique analysis option was selected and the minimum clique size was set to 3 individuals, although the smallest cliques in social network analysis can be a size of 2 or a dyad (Hanneman \& Riddle, 2005). Clique analysis aimed to examine connected groups of people beyond dyads because this would provide a more intuitive discussion about the dynamics of the network and how Caseworkers are connecting. 


\section{Latent Profile (class) Analysis}

Following on from social network analysis, Latent Profile Analysis was conducted on the social network data to determine if the way in which Caseworkers network with each other could be classified into groups. Essentially Latent Profile Analyses is a technique that seeks:

'to uncover hidden groups from observed data. They are similar to clustering techniques but more flexible because they are based on an explicit model of the data, and allow you to account for the fact that the recovered groups are uncertain' (Oberski, 2016, p. 1).

Latent profile analysis is a form of Latent Class Analysis which are statistical techniques used to create typologies, classes or groups from observed or measured data (Clark \& Muthén, 2009; Tein, Coxe, \& Cham, 2013). This technique was originally designed for use with dichotomous observed variables but has more recently developed to enable researchers to use continuous and ordinal data in the analysis (Tein et al., 2013), and the term used to describe this type of analysis is latent profile analysis or LPA. Latent Profile Analysis attempts to uncover sets of discrete subgroups or classes contained within a larger population based on the mean scores of continuous data (Clark \& Muthén, 2009; Oberski, 2016; Tein et al., 2013).

LPA does make a general assumption of local independence, in other words the values contained within each group are independent of each other. In a practical sense, the individuals contained within each group cannot be members of another group and membership is independent of the values contained within other classes.

This technique was adopted because it could be used to determine if the way Caseworkers were networking with each other in the larger Caseworker network could be quantified into connectivity profiles ${ }^{14}$. In other words, were groups of Caseworkers connecting with each other in similar ways at a level where they could be grouped together? Or were Caseworkers linking with each other in very different ways?

LPA was also chosen as an analytical method because the variables being examined were continuous variables (indegree and outdegree score). Not only was this approach appropriate given the type of variable being examined, but the results of this analysis could enhance our understanding of the dynamics of the Caseworker network. Furthermore, if patterns of networking are identified, a social and human capital

${ }_{14}$ In response to Q2a) Are there any distinct networking profiles which capture how Caseworkers are connecting with one another? 
perspective could be applied to explore if there is an association between these attributes and the ways in which Caseworkers are networking ${ }^{15}$.

Latent Profile Analysis was applied to the network data using MPlus (version 7.4). The criterion for distinguishing which model (or number of connectivity profiles) was the better fit for the observed data will now be examined. Results of this analysis are presented in Chapter Five.

The small sample size could result in bias, therefore bootstrapping (a form of resampling) was applied to test for correlations in the data when the LPA was being undertaken. This enhanced the confidence in the findings (Hayes, 2009; Roberts, 2014).

\section{Testing statistical significance}

To determine which results were statistically significant, tests of significance and goodness of fit indicators were used.

Tests of significance assumed to be significant at p-value $\leq 0.05$ (Bryman, 2008); this was applicable to all the quantitative analysis undertaken. However, while the p-value was of primary importance for most of the analytical techniques, for the Latent Profile Analysis, other general fit indices were also considered. These supplementary fit indices are studied when the p-values of different classes remain roughly the same and can be used to determine the number of classes (patterns of networking) that could be identified in the' social network data.

For goodness of fit indicators, aside from the P-value, the Bayesian Information Criterion (BIC) is the most commonly used 'goodness-of-fit statistic' (Field, 2009, p. 781 ) for model selections. This method is based on the 'maximum likelihood estimates of the model parameters for selecting the most parsimonious and correct model' (Tein et al., 2013, p. 4). In this thesis, the BIC was considered when determining the number of connectivity profiles (models) that best fits the data being examined. Typically, the model that has the lowest BIC value measure is the preferred model.

Along with the BIC, the Akaike Information Criterion (AIC) a common measure used to test the relative quality of model fit within a given data set. Essentially the AIC is a 'goodness-of-fit measure that is corrected for model complexity' (Field, 2009, p. 781). The

${ }^{15}$ In response to $\left.\mathrm{Q}^{2 b}\right)$ What individual factors help explain the differences in connectivity across the network? 
way the AIC works is that it is presented with a collection of models for the data (patterns of networking) and then estimates the quality of each model in relation to every other model it has been presented with and the estimated number of parameters to determine which model better fits the data being examined. Typically, the model with the lower AIC value has a better fit and is the preferred value for model selection.

Both the AIC and BIC values are not interpretable as a single value for one model but rather enables comparisons between models to determine which model or number of profiles is a better fit for the observed data being examined (Field, 2009).

Entropy is a 'measure of aggregated classification uncertainty' (Tein et al., 2013, p. 4) and is measured on a zero to one scale with a value of one indicating that individuals are perfectly classified into the latent groups or classes. Overall, a higher entropy value indicates a better classification of individuals (Clark \& Muthén, 2009). In contrast to $\mathrm{AIC}$ and BIC, the entropy value does evaluate the quality of the class measurement or delineation. Entropy values that are approaching 1 indicates that there is a clear delineation or separation of classes (Asparouhov \& Muthén, 2014; Burholt, Dobbs, \& Victor, 2016).

The adequacy of model fit was also determined using the Vuong-Lo-Mendell-Rubin adjusted likelihood ratio test and the boot-strapped parametric likelihood ratio test (Burholt et al., 2016; McLachlan \& Peel, 2000). Both tests compare the model with a number of classes $(K)$ to a model with that number of classes minus 1 (Tein et al., 2013).

\section{Capturing “Capitals” and Contexts}

The variables in the online questionnaire were coded to capture the Caseworkers' human and social capital attributes, and the contextual factors affecting their work. They were then used to test their association with social network variables (normalised indegree and outdegree scores), and the frequency and breadth of connections Caseworkers have developed with external organisations. The results of this analysis are presented in Chapters Five and Six.

\section{Human and social capital variables}

Professional/Vocational qualifications: the question 'Do you have any other professional or vocational qualification?' provided an opportunity for participants who replied 'yes' to the question to indicate what these were. Text answers were coded into seven dichotomous variables which are not mutually exclusive including: Finance; Health; 
Management; IT; Practical; Energy; NVQ (see Appendix Item 11 for details of variable coding).

Type of training received: five variables were derived from the responses to the question 'Please let us know about any training you have received since working for Care $\mathcal{E}^{\circ}$ Repair in your role as Caseworker, including any certified qualifications (e.g. First Aid)?' Textual answers were recoded into 5 dummy variables including Health and safety; Housing and energy; Finance; Personal skills; Interpersonal (see Appendix Item 11 for details of variable coding).

However, these variables are not mutually exclusive because Caseworkers can attend more than one type of training course throughout their time in post as a Caseworker. Additionally, another variable was created to count the number of training courses that Caseworkers have attended since beginning their job role as a Caseworker.

Previous job sector: twenty-one variables (derived from the Census) were generated from the question 'Please indicate which sectors you have previously worked in prior to taking on the role of Caseworker'. Answers were recoded into six variables: Public service Healthcare; Public service; Business; Practical/Technical; Charity and Voluntary; Customer Service (see Appendix Item 11 for details of variable coding). These variables are not mutually exclusive as Caseworkers can have had a varied working past spanning many job sectors.

Previous work experience: variables representing 'previous work experience' were created using the data arising from the question which asked respondents to 'Indicate which of the following categories describe your past work experience?' Textual answers were recoded into four variables: Managerial; Administrative; Customer service; Practical (see Appendix Item 11 for details of variable coding).

Job title: four job title variables were coded using textual answers from the question 'What is your job title?' These include: Caseworker; Technical Caseworker; Social care Caseworker; Specialist Caseworker (see Appendix Item 11 for details of variable coding).

\section{Contextual variables}

In addition to capturing human and social capital variables the online self-completion questionnaire also captured contextual variables. 
Core funding: a variable representing sources of funding for Caseworkers was created in response Question 12 (which asked, "Is your role funded by 'core funding'?"). This included Yes-funded by Welsh Government; Other type of funding (see Appendix Item 11 for details of variable coding).

Agency location; using the question 'In which Care \& Repair Agency are you employed?' One variable comprised of fifteen categories which were coded based on the textual answers provided by respondents. These include: Blaenau Gwent; Bridgend; Caerphilly, Cardiff and the Vale; Carmarthenshire; Conwy and Denbighshire; Greynedd a Mon; Merthyr Tydfil; Monmouthshire and Torfaen; Neath Port Talbot; Newort, North East Wales; Powys; Rhondda Cynon Taf; Swansea; West Wales (see Appendix Item 11 for details of variable coding).

Issues on the ground: thirteen variables were coded using the textual data from the question 'From the list below, please indicate the factors that correspond to the sorts of difficulties you may have experienced in carrying out your work as a Caseworker'. These included: Organisations and services are reluctant to work together; Difficulties in accessing. clients who live in remote areas; ICT problems; Client reluctant to accept help; Client's objections to giving personal details; Client is unhappy about changing their home environment; Couples disagree between themselves about the support needed; Client's family objects to Cङ्RC interventions; Limited finds available to help support client's needs; Changes to benefits eligibility criteria; Language barriers; Organisations restructuring; Time needed to complete administrative tasks (see Appendix Item 11 for details of variable coding). Like most of the variables discussed, these variables were non-mutually exclusive as Caseworkers could experience many of these issues.

\section{Linking with external organisations Frequency and breadth of external links}

Data collected from the online questionnaire captured the breadth and depth of links made by the Caseworkers with organisations outside of Care \& Repair Cymru ${ }^{16}$. Two variables were created; one to reflect how often Caseworkers link with each type of external organisation (frequency) and one to reflect how many (breadth) different organisations that Caseworkers link with.

${ }^{16}$ As a response to research question 3a) What types of services do Caseworkers connect with across Wales? 
Data for these variables came from the section D of the questionnaire (see Appendix item 3), which asked about six main types of organisations with a total of sixteen predefined categories of organisations they linked with. It also asked about the frequency of contact.

The categories included (1) Financial assistance organisations, (2) Fundraising on behalf of clients-Businesses, (3) Fundraising on behalf of clients - Public sector, (4) Fundraising on behalf of clients - Charities, (5) Fundraising on behalf of clients - Professional/ Occupational, (6) Referrals-Befriending/ community support, (7) Referrals-Re-enablement, (8) ReferralsHealth, (9) Referrals- Personal care, (10) Referrals-Domestic help, (1 1) Digital inclusion, (12) Healthy homes check- Fuel poverty, (13) Healthy homes check- Fuel safety, (14) Healthy homes check-Slips, trips and falls, (15) Healthy homes check-Security of the home, (16) Home Maintenance.

The frequency of contact variables were coded as (1) Daily contact, (2) Weekly contactless than daily but at least weekly, (3) Monthly contact- less than weekly but at least monthly, (4) One-off contact- less than monthly but at least once, (5) No contact.

Count variables were coded to reflect the breadth of connections Caseworkers made within each category. They represent the sum of organisations that Caseworkers indicate they link with. They were not normalised because the analysis could be undertaken without issue and the results could be clearly interpreted and understood without normalising the data.

\section{Statistical tests}

A range of statistical tests were used to assess the association between individual and contextual factors (social and human capital and contextual variables) and the Caseworker network (normalised indegree and outdegree measures). The purpose of these tests was to determine if individual or contextual factors could explain the different ways Caseworkers connect with each other. Other tests were used to assess associations between the same individual and contextual factors and frequency and breadth of connections with external organisations that Caseworkers build to deliver services to their clients.

\section{Bivariate analyses}

The first analytical technique used in this thesis was one-way analysis of variance. ANOVAS were used to explore if there was an association between the mean scores 
of normalised indegree and outdegree categorical or nominal variables that was gathered in the Caseworker questionnaire ${ }^{17}$. ANOVA tests were also used to examine if there were associations between the breadth of connections that Caseworkers were making, and categorical or nominal variables. Tukeys HSD post-hoc tests were also used to compare pairs of group means enabling the researcher to look for differences between groups.

Spearman's rho correlation tests were used to correlate ordinal variables with continuous or interval variables (Bryman, 2008). This test was used to correlate normalised indegree and outdegree scores with educational qualification (an ordinal variable). The educational qualification variable was also correlated with the frequency of connections with organisations to determine if this individual factor influences how Caseworkers network with each other and connect with organisations outside of Care \& Repair Cymru.

Additionally, Chi-Square test of independence was undertaken to determine if group membership (derived from Latent Profile Analysis) was associated with individual or contextual variables. In other words, this test was used to determine if differences in the Caseworkers' connectivity was associated with individual or contextual factors.

\section{Qualitative data preparation and analyses Transcription}

The interviews and focus groups were audio-recorded, and the data had to be transformed into a format which could be utilised in an analysis. In total, 1,442.15 minutes of focus group and interview data was collected.

The Chief Officer interviews focus group data, in addition to two of the client interviews were transcribed by the $\mathrm{PhD}$ candidate while the remaining seven interviews were transcribed by a private transcription service. Once the interviews and focus groups were transcribed, the documents were uploaded into NVivo 10 for the purposes of coding the data and to identify themes.

\footnotetext{
${ }_{17}$ The nominal variables used in the ANOVAS to answer research question $2 \mathrm{~b}$ include agency location, gender, Caseworker job role, funding, training courses, past work experience and previous job sector employment. The same nominal variables were also used to answer research question $3 \mathrm{~b}$ in addition to contextual variables including agency location and issues faced by Caseworkers on the ground.
} 


\section{Thematic analysis and coding}

Thematic analysis is a method of analysis used by researchers which provides a framework for identifying and exploring recurrent patterns and themes (Braun \& Clarke, 2006) that are present in qualitative data and which have been identified through transcript coding (Sarantakos, 2005). It reflects an inductive approach (Joffe, 2012) because generally speaking, themes are not prescribed beforehand by the researcher but emerge from the data (Sarantakos, 2005). Thematic analysis aligns with the mixed method approach adopted in this thesis, and an interpretive phenomenological perspective which seeks to examine how individuals attach meaning to life events through their own experiences and individual understanding of a phenomenon (Smith, Flowers, \& Larkin, 2009). In the context of this thesis, thematic analysis of qualitative transcripts was adopted because it offers the opportunity to examine the subtler dimensions to the caseworkers' role and networking activities which are not captured through the statistical analysis and social network mapping gives insights into client perspectives on the impact that caseworker interventions have on their lives.

\section{How the coding framework was developed}

Transcripts from qualitative interviews and the open-ended responses from the caseworker online questionnaire were initially explored by reading and re-reading them so that the $\mathrm{PhD}$ candidate could familiarise herself with the content and have a sound grasp of the broader context within which themes were emerging (Welsh, 2002). Initial manual coding of prominent words and themes was then undertaken, and this also helped to highlight and link or cross-link different parts of one or more transcripts, where common words and themes had been identified. Then coding categories were developed and applied using NVivo (Mason, 2010). Although the software cannot help as an analytical tool, it does facilitate coding, searching and annotating transcripts when large volumes of qualitative data are involved (Burnard, Gill, Stewart, Treasure, \& Chadwick, 2008; Hoover \& Koerber, 2011).

The first step of the coding with NVivo was to conduct a word query search of the qualitative transcripts, taking into account some of the words, terms and themes identified manually. Due to time and financial constraints, this was done in more depth for the interviews with clients, and a list of the most commonly used words throughout the interviews was generated. The word query search was then expanded to include synonyms, and the words were then grouped together to produce thematic categories. For instance, the word 'knows' its synonyms 'know', knowing' and 'knows' arose 354 times and the word 'worrying' and the related words of 'worried,' 'worries' 
and 'worry', occurred 53 times throughout the 9 client interviews transcripts analysed.

Once the commonly used words were identified, the transcripts were examined further to substantiate and refine the list of possible themes that could be included in the coding framework such as 'alleviating worry or stress' for 'worrying' and 'expertise and advice of the Caseworkers' for 'knows'. For instance, after searching and coding for 'worrying' in the qualitative data for each transcript, the word and its synonyms were then identified across transcripts and coded into a cross-cutting theme (see Table 4.1 for examples).

Table 4.1- Illustration of creation of themes from word query search

\begin{tabular}{|c|c|c|}
\hline Words & Theme & Example \\
\hline $\begin{array}{l}\text { 'worrying' 'worried,' } \\
\text { 'worries' and 'worry' } \\
\text { And } \\
\text { 'stress', 'strain', } \\
\text { 'stressed', 'stressing' }\end{array}$ & $\begin{array}{l}\text { Alleviating worry or } \\
\text { stress }\end{array}$ & $\begin{array}{l}\text { '...it has made my life a lot easier... } \\
\text { they've taken the pressure off me; a lot } \\
\text { of pressure and they've done things for } \\
\text { me which is very good' [Participant } \\
6] \text {. }\end{array}$ \\
\hline $\begin{array}{l}\text { knows', 'know', } \\
\text { knowing' and 'knows' }\end{array}$ & $\begin{array}{l}\text { Practical knowledge } \\
\text { and expertise }\end{array}$ & $\begin{array}{l}\text { 'Things we, we didn't have a clue } \\
\text { about; she guided us through } \\
\text { everything you know?' [Participant } \\
\text { 1a]. }\end{array}$ \\
\hline $\begin{array}{l}\text { 'Like', 'care', 'careful', } \\
\text { 'carefully', 'cares', and } \\
\text { 'caring,' }\end{array}$ & al supl & $\begin{array}{l}\text { '... she's back and forth asking me } \\
\text { how I am, how I'm getting on, do I } \\
\text { need help? She's there... she has been } \\
\text { there if I wanted to talk to somebody. } \\
\text { She said, "We're here with you now, } \\
\text { all the time. This is our job," and they } \\
\text { go beyond their job actually...there is } \\
\text { always that- I don't know- the } \\
\text { connection there with them. It's not } \\
\text { like somebody coming in and just } \\
\text { saying, "Yes, we'll do this, and we'll } \\
\text { do that." They do care, and they will } \\
\text { do it' [Participant } 6] \text {. }\end{array}$ \\
\hline
\end{tabular}

As indicated in Table 4.2 several themes about the caseworker and the services they provide were identified. Additional themes emerged which capture clients' perspectives on $\mathrm{C} \& \mathrm{RC}$ as an organisation, the Technical Officer and the external service providers whom Care \& Repair Cymru agencies link with. Others alluded to the more functional aspects of the service e.g. the processes involved in organising and carrying out adaptations. However, other themes were more subjective or affective and this provided the basis for considering the relevance of adopting an 
interpretive framework of findings which draws on "emotion work" research (see Chapter 2 for a review of emotion work theory).

Table 4.2- Illustration of the themes that were identified in the client interviews

\begin{tabular}{|c|c|c|c|c|c|}
\hline Themes & Sub-themes & $\begin{array}{l}\text { C\&RC as an } \\
\text { organisation }\end{array}$ & CWs & TOs & $\begin{array}{l}\text { External } \\
\text { service } \\
\text { providers }\end{array}$ \\
\hline $\begin{array}{r}\text { Reassurance } \\
\text { and } \\
\text { confidence }\end{array}$ & $\begin{array}{l}\text { Care \& Repair } \\
\text { provides } \\
\text { reassurance } \\
\text { - Giving } \\
\text { Confidence }\end{array}$ & $\mathrm{X}$ & $\begin{array}{l}X \\
X\end{array}$ & $\mathrm{X}$ & \\
\hline $\begin{array}{r}\text { Trustworthy } \\
\text { and } \\
\text { professional } \\
\text { service }\end{array}$ & $\begin{array}{l}\text { - Honest, reliable } \\
\text { and } \\
\text { trustworthy } \\
\text { referrals } \\
\text { - } \text { Benefit claims } \\
\text { - } \text { Adaptations } \\
\text { - } \begin{array}{l}\text { Practical } \\
\text { knowledge and } \\
\text { expertise }\end{array}\end{array}$ & $\mathrm{X}$ & $\begin{array}{l}X \\
X \\
X \\
X\end{array}$ & $\begin{array}{l}\mathrm{X} \\
\mathrm{X}\end{array}$ & $\mathrm{X}$ \\
\hline $\begin{array}{r}\text { Follow-up } \\
\text { service } \\
\text { outcomes }\end{array}$ & $\begin{array}{l}\text { Expectations/ } \\
\text { developing } \\
\text { person centred } \\
\text { service/ } \\
\text { proactive } \\
\text { - Emotional } \\
\text { Support } \\
\text { - Alleviating } \\
\text { worry or stress }\end{array}$ & $\mathrm{X}$ & $\begin{array}{l}X \\
X \\
X\end{array}$ & $\mathrm{X}$ & \\
\hline
\end{tabular}

To ensure that the coding framework and identification of themes were valid and accurately reflected the meaning of the data, 'multiple coding' (Barbour, 2001) or cross checking of data was used to enhance the validity of the results. Multiple coding is the qualitative equivalent of inter-rater reliability which is used in quantitative research (Barbour, 2001). As a process, it does not involve replication of results, but rather it involves independent researchers cross checking the 'coding strategies and interpretation of data' (Ibid, p. 1116).

In terms of this thesis, an independent researcher was provided with the coding framework and clean versions of a selection of 3 client interview transcripts and was asked to code them. The coded transcripts were then compared with those coded by the $\mathrm{PhD}$ candidate. Dissimilarities in the use of coding to identify the theme of "emotion work" were picked up and the coding framework refined, and transcripts recoded (see Appendix item 12 for illustration part of the client interview coding 
matrix). This enhanced the rigour of the results of the qualitative analysis (Saldaña, 2015).

\section{Chapter summary}

This chapter has outlined the research design, data collection and analytical techniques used in this thesis. First, the exploratory sequential mixed methods design of the research project was outlined before discussing the study population, recruitment, sampling procedures, and response rates of participants. The chapter then examined each of the research methods used to gather data throughout the research process. How the data was prepared was then referenced including examinations of how the variables and measures used in the analysis were created, in addition to transcribing and identifying themes in the qualitative data. The sorts of tests used, and the variables used in the quantitative analysis and social network analysis was discussed. The following chapter will present the findings from the social network analysis. 


\section{CHAPTER FIVE}

\section{Presentation of Findings - Part One: Networking within Care \& Repair}

This chapter will present the key findings that have arisen out of the social network analysis which examined how the Caseworkers were networking with each other. The results presented here address four research questions (1a, 1b, 2a and 2b) which explored: the types of connections that Caseworkers were making; if they networking as groups; if there were dominant patterns of networking between Caseworkers; and if there were any individual capitals, which were associated with differences in how the Caseworkers were networking within Care \& Repair Cymru.

\section{Descriptive analysis of study population}

First a description of the composition of the Caseworker study population (comprised of 55 out of 78 possible individuals see Chapter Four for more detailed response rate narrative). Appendix item 13 gives an overview of the frequency distributions that represent the contextual, socio-demographic and capital characteristics of those who replied to the questionnaire (55 Caseworkers out of 78). These characteristics represent some of the independent variables used in the analyses.

In terms of the regional distribution, $31 \%(n=17)$ of the Caseworkers were in a North Wales Agency (number of agencies $n=3)$, while $63.8 \%(n=35)$ were located and operating in South Wales (number of agencies $n=11)$, and the remainder $5.5 \%(n=3)$ were situated in mid Wales (number of agencies $n=1$ ).

The sample was predominately female; $87.3 \%(n=48)$ and appeared to be a more 'mature' work force where the modal age was the 40-49 years of age group. Only $9.1 \%$ $(n=5)$ of the study population were under the age of 30 years, $17 \%$ were aged between 30-39 years, and 35\% were aged between 40-49 and 50-59 years, with 4\% aged 60 years or more. At the time of data collection, around three quarters (75.9\%) of the study population were aged over $40(n=41)$ and around two-fifths $(40.7 \%)$ were aged 50 years or more $(\mathrm{n}=22)$.

In terms of educational attainment, $40 \%$ have higher education qualifications $(n=22)$, $32.7 \%$ have a further education qualification $(n=18)$, just over one fifth of the Caseworkers (21.8\%) have a GCSE/ O level qualification $(\mathrm{n}=12)$, and the remainder either have an apprenticeship (3.6\%), or no qualifications $(1.8 \%)$. 
About half (50.9\%) of all the participating Caseworkers have no professional or vocational qualification (e.g. NVQ, City \& Guilds). In decreasing order of frequency, the most prevalent type NVQ' vocational qualifications are held by $21.8 \%(n=12)$, followed by 'practical' vocational qualifications (including City and Guilds, PGCE and CELTA), held by $18.2 \%(n=10)$, while $16.4 \%(n=9)$ hold a Health and Social Care qualification, such as Trusted Assessor training. The mean number of training courses attended is $9.15(\mathrm{SD}=9.36)$. Given that just over half of the study population have no vocational qualifications, this may indicate that as a group they require more training to enhance or develop their capitals.

When examining the frequency of previous job sectors experiences, more than half of the Caseworkers (56.4\%) have worked in the 'Public Service sector' (e.g. education, public administration and uniformed services). This is followed in order of decreasing frequency by 'customer service sector' e.g. hospitality, leisure and travel, or retail and customer service sectors, and around half $(50.9 \%, \mathrm{n}=28)$ of the sample have worked in this job sector. The third most prevalent work sector previously occupied is the 'charity and voluntary sector' $(43.6 \%, \mathrm{n}=24)$.

Almost three quarters $(74.5 \%)$ of Caseworkers $(n=41)$ have the job title of the 'Caseworker'; however, $10.9 \%(\mathrm{n}=6)$ are a 'specialist Caseworkers', for example 'managing better Caseworkers', 'affordable warmth Caseworkers' and 'critical prevention Caseworkers'. Therefore, while much of the Caseworkers complete tasks and deliver services as part of the Wales-wide Caseworker service, there a variety of Caseworker roles which are more specialised and focused on achieving a specific agenda and meeting very specific needs e.g. social care or technical roles.

Just over half, $(52.7 \%, \mathrm{n}=29)$ of the Caseworkers have been in the role for 5 years or more. The next most prevalent group are the 'new starters group' or those who have been in the role for less than 6 months, and those who have been in post between 12 years (12.7\% for each group). This means that Care \& Repair Cymru have a 'seasoned' workforce made up of individuals who have been in their roles for at least five years.

Around two-thirds $(67.3 \%, \mathrm{n}=37)$ of the study population of Caseworkers have core funding from the Welsh Government, and almost three quarters, $(74.5 \%, \mathrm{n}=41)$ work full time in the organisation. Finally, around two thirds $(65.5 \%, \mathrm{n}=36)$ of the sample work across several Welsh counties. This suggests that Caseworkers may be working 
collaboratively with other agencies across county boundaries to deliver services to older clients.

In sum, the Caseworkers represent a workforce that has a diverse range of background, skills and knowledge. For instance, over half of them have been in post for at least 5 years which suggests that these individuals have been able to build their capital around this role. Similarly, as evidenced in the previous job sector frequency distribution their occupational background is quite mixed. This infers that they bring a range of human and social capitals into the role.

\section{How Caseworkers are connecting with each other}

Figure 5.1 provides a graphical representation of the Caseworker network depicting which individuals are connecting to one another across Wales at the time of data collection, between February and September 2016.

At first glance, the shape of the network indicates that Caseworkers are networking in different ways, because every participant is not connected to every other individual in the network. Some individuals have more connections than others and others have no connections to the network. For example, the large purple-blue rectangle (RCT5) towards the middle of the network suggest that this individual has the largest number of connections, whilst the small white squares on the periphery of the network (e.g. C\&V5), suggest that these individuals receive only one connection from others and therefore have the fewest number of links. What the sociogram also shows is that connections are not constrained to geographic location, and that individuals from different areas and regions of Wales are connecting with each other. This suggests that other factors (rather than location) influence how Caseworkers are connecting with each other and why some have more connections than others. For instance, some individuals may have specific skills, knowledge or contacts which others wish to access. This will be explored in greater depth later in this chapter, where the individual characteristics (social and human capital variables) are tested to determine if any of them are associated with the differences in connectivity across the network.

\section{The numbers of connections Caseworkers make}

As noted in Chapter Four (Measures used in SNA section), 'degree centrality' measures the number of connections or ties that each Caseworker has in terms of outdegree and indegree scores. In other words, the outward facing ties are those that each individual is initiating themselves, and inward facing ties are those which are received from 
others linking with them. The betweenness centrality measure provides insight into the potential of an individual to act as a bridge to connect otherwise unconnected individuals.

Table 5.1- Descriptive Statistics for degree centrality scores for 55 Caseworkers with complete data ${ }^{18}$

\begin{tabular}{r|llll}
\hline Measure & 1. Outdegree & 2. Indegree & 3. NrmOutDeg & 4. NrmInDeg $^{\mathbf{2 0}}$ \\
\hline Mean (SD) & $3.55(4.20)$ & $2.67(2.14)$ & $0.05(0.06)$ & $0.04(0.03)$ \\
Minimum & 0.000 & 0.00 & 0.00 & 0.00 \\
Maximum & 15.00 & 10.00 & 0.20 & 0.13 \\
\hline
\end{tabular}

Results (Table 5.1) show that Caseworkers' outdegree scores range from 0-15 with a mean outdegree score of $\mathrm{m}=3.55(\mathrm{SD}=4.20)$. The indegree scores range from $0-10$ and the mean indegree score is $\mathrm{m}=2.67(\mathrm{SD}=2.14)$.

Examining the different individual Caseworker indegree and outdegree scores by agency location, it is evident that they are developing different patterns of interaction, as some have higher individual scores of indegree and outdegree than others. For instance, RCT5 has established 14 outward facing ties (the highest outdegree score of all the Caseworkers in the network) but has only 5 incoming ties or links which their colleagues have initiated. In contrast, Con\&Den 1 has 10 incoming ties (the greatest indegree score of any Caseworker in the network) but has reported only 1 outward facing tie with other Caseworkers.

Overall, patterns of interaction indicate that RCT5 had the greatest overall degree score with 19 connections (see Table 5.2, Column 5), and is placed at the centre of the network as illustrated in Figure 5.1 (this is a sociogram or social map which visually represents the degree centrality measures identified in Tables 5.1 and 5.2). RCT5 is represented by a lilac box in the centre of the network indicating they have the most direct links with other Caseworkers across the network. In contrast, there are 4 individuals located at the periphery of the network with an overall centrality

\footnotetext{
${ }^{18}$ Some of the Caseworkers included in the socio-gram did not respond to the questionnaire but other Caseworkers indicated that they linked with these individuals. To omit these individuals from the sociogram would cause bias in the results. Also, the indegree scores of the non-responder Caseworkers were excluded from the statistical analyses to prevent bias. Hence, completed data in this instance refers to the Caseworkers who responded to the questionnaire.

19 Column 3 represents mean normalised outdegree measure for the 55 Caseworkers which is calculated by the following formula:

Outdegree measure x 100 / (Number of individuals in network-1)

After calculating this measure for each respondent, a mean average normalised outdegree score was computed.

${ }^{20}$ Column 4 represents the mean normalised indegree measure for the 55 Caseworkers which was computed using the formula above.
} 
score of 1 (including WW1, Pow4, NPT4 and Carm3). Furthermore, there are 8 Caseworkers who are not connected to the network at all who have an overall centrality score of o indicating that they are not networking with other Caseworkers to achieve the aims of their job roles. These have been omitted from the sociogram for reasons of clarity, but they are discussed later in the chapter. 
Table 5.2- Summary of the individual indegree and outdegree scores of

Caseworkers

\begin{tabular}{|c|c|c|c|c|c|}
\hline $\begin{array}{r}\text { Caseworker } \\
\text { identifier }\end{array}$ & $\begin{array}{l}1 . \\
\text { Outde } \\
\text { gree }\end{array}$ & $\begin{array}{l}\text { 2. } \\
\text { Indegree }\end{array}$ & $\begin{array}{l}\text { 3. } \\
\text { Normalized } \\
\text { Outdegree }\end{array}$ & $\begin{array}{l}4 . \\
\text { Normalized } \\
\text { Indegree }\end{array}$ & $\begin{array}{l}\text { 5. Total } \\
\text { number } \\
\text { of ties }\end{array}$ \\
\hline$B G 1$ & 3.00 & 4.00 & 0.04 & 0.05 & 7 \\
\hline$B G^{2}$ & 2.00 & 4.00 & 0.03 & 0.05 & 6 \\
\hline Caer 2 & 3.00 & 1.00 & 0.04 & 0.01 & 4 \\
\hline Caer 3 & 11.00 & 6.00 & 0.15 & 0.08 & 17 \\
\hline Bridge 1 & 0.00 & 1.00 & 0.00 & 0.01 & 1 \\
\hline Bridge 2 & 4.00 & 0.00 & 0.05 & 0.00 & 4 \\
\hline Bridge 3 & 4.00 & 3.00 & 0.05 & 0.04 & 7 \\
\hline$C \mathcal{E}^{2} V 1$ & 0.00 & 0.00 & 0.00 & 0.00 & $\mathrm{O}$ \\
\hline$C \mathcal{E}^{2} V 6$ & 0.00 & 0.00 & 0.00 & 0.00 & $\mathrm{O}$ \\
\hline$C \mathcal{E}^{2} V 8$ & 0.00 & 0.00 & 0.00 & 0.00 & $\mathrm{O}$ \\
\hline$C छ ి V 9$ & 0.00 & 0.00 & 0.00 & 0.00 & $\mathrm{O}$ \\
\hline$C \mathcal{G}^{2} V 10$ & 0.00 & 0.00 & 0.00 & 0.00 & $\mathrm{O}$ \\
\hline Carm 1 & 7.00 & 4.00 & 0.09 & 0.05 & 11 \\
\hline Carm 2 & 0.00 & 2.00 & 0.00 & 0.03 & 2 \\
\hline Carm 3 & 0.00 & 1.00 & 0.00 & 0.01 & 1 \\
\hline Carm 4 & 3.00 & 5.00 & 0.04 & 0.07 & 8 \\
\hline ConE Den 1 & 1.00 & 10.00 & 0.01 & 0.13 & 11 \\
\hline ConE Den 2 & 1.00 & 2.00 & 0.01 & 0.03 & 3 \\
\hline ConẼ Den 3 & 9.00 & 5.00 & 0.12 & 0.07 & 14 \\
\hline ConE Den 4 & 3.00 & 1.00 & 0.04 & 0.01 & 4 \\
\hline Gry & 0.00 & 0.00 & 0.00 & 0.00 & $\mathrm{O}$ \\
\hline Gry & 1.00 & 2.00 & 0.01 & 0.03 & 3 \\
\hline Gwy@A Ang 4 & 2.00 & 1.00 & 0.03 & 0.01 & 3 \\
\hline MonETor 1 & 3.00 & 3.00 & 0.04 & 0.04 & 6 \\
\hline MonETor 3 & 5.00 & 1.00 & 0.07 & 0.01 & 6 \\
\hline MonETor 4 & 3.00 & 5.00 & 0.04 & 0.07 & 8 \\
\hline NPT 2 & 3.00 & 6.00 & 0.04 & 0.08 & 9 \\
\hline NPT 3 & 0.00 & 2.00 & 0.00 & 0.03 & 2 \\
\hline Nport 1 & 0.00 & 3.00 & 0.00 & 0.04 & 3 \\
\hline$N E W 1$ & 0.00 & 3.00 & 0.00 & 0.04 & 3 \\
\hline$N E W 2$ & 1.00 & 5.00 & 0.01 & 0.07 & 6 \\
\hline NEW 3 & 9.00 & 8.00 & 0.12 & 0.11 & 17 \\
\hline$N E W 4$ & 2.00 & 3.00 & 0.03 & 0.04 & 5 \\
\hline$N E W 5$ & 0.00 & 3.00 & 0.00 & 0.04 & 3 \\
\hline NEW 6 & 7.00 & 2.00 & 0.09 & 0.03 & 9 \\
\hline$N E W 7$ & 0.00 & 3.00 & 0.00 & 0.04 & 3 \\
\hline$N E W 8$ & 11.00 & 3.00 & 0.15 & 0.04 & 14 \\
\hline NEW 9 & 0.00 & 2.00 & 0.00 & 0.03 & 2 \\
\hline Pow 1 & 0.00 & 0.00 & 0.00 & 0.00 & $\mathrm{O}$ \\
\hline Pow 2 & 0.00 & 0.00 & 0.00 & 0.00 & $\mathrm{O}$ \\
\hline Pow 4 & 0.00 & 1.00 & 0.00 & 0.01 & 1 \\
\hline$R C T 1$ & 3.00 & 4.00 & 0.04 & 0.05 & 7 \\
\hline$R C T 2$ & 11.00 & 2.00 & 0.15 & 0.03 & 13 \\
\hline$M T 1$ & 8.00 & 3.00 & 0.11 & 0.04 & 11 \\
\hline Swan 1 & 2.00 & 4.00 & 0.03 & 0.05 & 6 \\
\hline Swan 2 & 6.00 & 3.00 & 0.08 & 0.04 & 9 \\
\hline Swan 3 & 6.00 & 6.00 & 0.08 & 0.08 & 12 \\
\hline$W W 1$ & 0.00 & 1.00 & 0.00 & 0.01 & 1 \\
\hline$W W 4$ & 3.00 & 1.00 & 0.04 & 0.01 & 4 \\
\hline$W W 7$ & 15.00 & 3.00 & 0.20 & 0.04 & 18 \\
\hline Swan 4 & 6.00 & 3.00 & 0.08 & 0.04 & 9 \\
\hline$N P T 4$ & 0.00 & 1.00 & 0.00 & 0.01 & 1 \\
\hline$R C T 5$ & 14.00 & 5.00 & 0.18 & 0.07 & 19 \\
\hline Gryes'Ang 6 & 10.0 & 3.00 & 0.13 & 0.04 & 13 \\
\hline Nport 4 & 13.00 & 3.00 & 0.17 & 0.04 & 16 \\
\hline
\end{tabular}


Figure 5.1-Sociogram of the Wales-wide Caseworker network

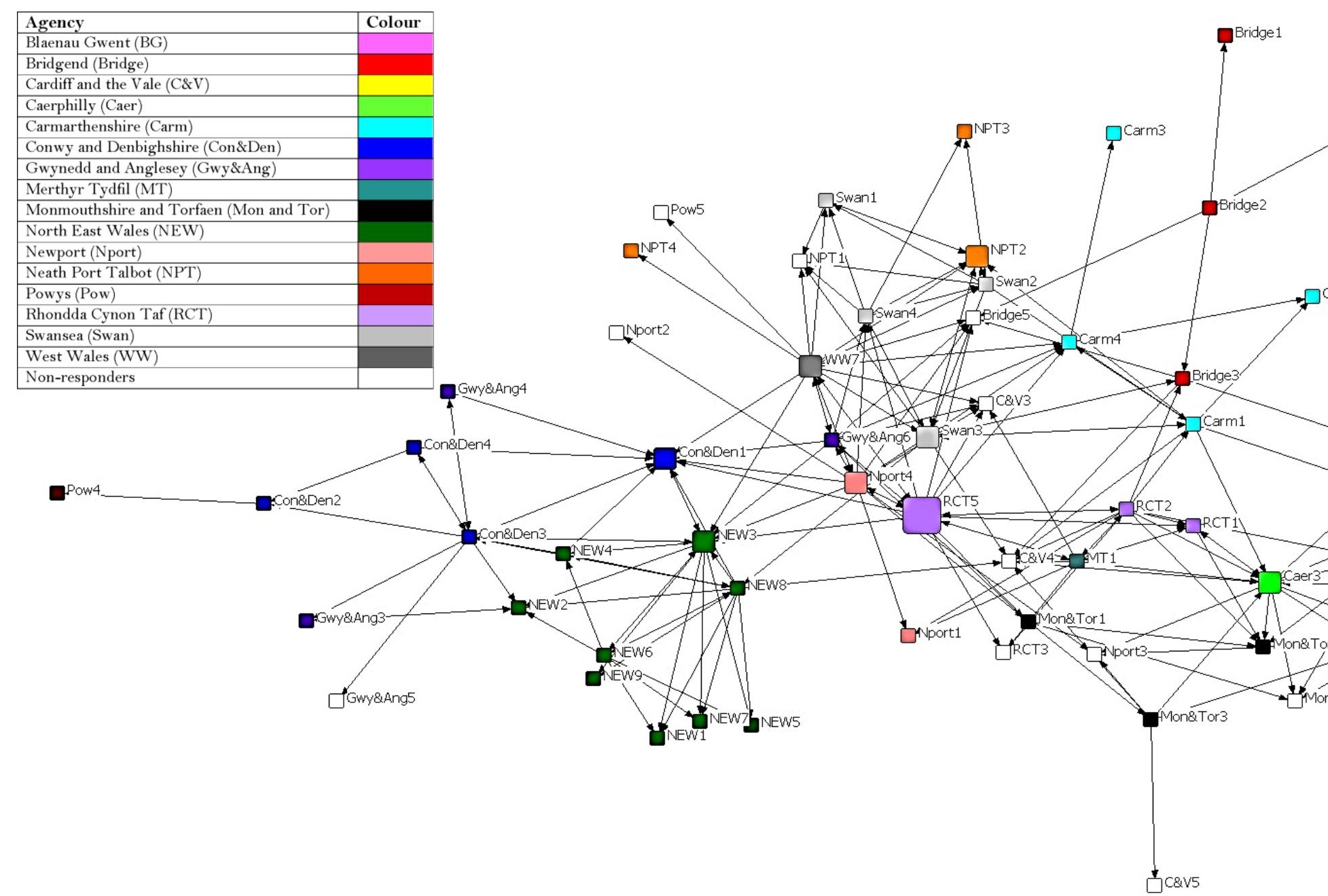




\section{Caseworkers as bridges or "connectors"}

Results for this analysis show a mean betweenness centrality score of $\mathrm{m}=58.44(\mathrm{SD}=$ 1 10.12) (Table 5.3). This means that in the Caseworker network, individuals have the potential to act as a connecting agent or bridge on average 58.44 times.

Table 5.3- Descriptive Statistics for Betweenness centrality scores for $\mathbf{5 5}$ Caseworkers with complete data

\begin{tabular}{r|ll}
\hline Measure & Betweenness $^{21}$ & NrmBetweenness $^{22}$ \\
\hline Mean $(\boldsymbol{S D})$ & $58.44(110.12)$ & $1.03(1.93)$ \\
Minimum & 0.00 & 0.00 \\
Maximum & 432.97 & 7.60 \\
\hline
\end{tabular}

The minimum and maximum betweenness centrality scores are 0.00 and 432.97 respectively. The difference between these scores suggests that some Caseworkers occupy more strategic positions in their network, which enables them to act as a bridge or connecting agent between network members who are otherwise unconnected.

${ }^{21}$ This column represents the mean betweenness centrality score of the 55 Caseworker participants and represents the mean number of times participants fall on the shortest paths between two otherwise unconnected individuals. This indicates the potential of individuals to act as bridges or brokers between other unconnected participants.

${ }^{22}$ This is a normalised measure of the mean betweenness centrality produced by the UCINET software. 
Table 5.4- Summary of the individual betweenness centrality scores of Caseworkers

\begin{tabular}{|c|c|c|}
\hline Caseworker identifier & Betweenness score & $\begin{array}{l}\text { Normalised betweenness } \\
\text { score }\end{array}$ \\
\hline Caer3 & 432.97 & 7.60 \\
\hline$M T 1$ & 356.45 & 6.25 \\
\hline$R C T 5$ & 354.27 & 6.22 \\
\hline$R C T 1$ & 324.36 & 5.69 \\
\hline Sswan3 & 293.74 & 5.15 \\
\hline Carm1 & 293.53 & 5.15 \\
\hline$N E W 3$ & 190.67 & 3.35 \\
\hline Conฮ̊Den\} & 171.08 & 3.00 \\
\hline$N E W_{8}$ & 162.5 & 2.85 \\
\hline Bridge3 & 74.78 & 1.31 \\
\hline Mon ฮాTor3 & 74.52 & 1.31 \\
\hline NPT2 & 71.67 & 1.26 \\
\hline$W W 7$ & 61.83 & 1.08 \\
\hline Swan2 & 57.41 & 1.01 \\
\hline Mon & 49.65 & 0.87 \\
\hline$W W 4$ & 40.00 & 0.70 \\
\hline$R C T 2$ & 36.13 & 0.63 \\
\hline Carm4 & 34.77 & 0.61 \\
\hline Swan4 & 33.83 & 0.59 \\
\hline ConछDen2 & 28.00 & 0.49 \\
\hline Nport4 & 26.34 & 0.46 \\
\hline$N E W_{2}$ & 12.5 & 0.22 \\
\hline MonछTTor4 & 10.12 & 0.18 \\
\hline Conฮ๋Den 1 & 7.17 & 0.13 \\
\hline$B G 1$ & 3.78 & 0.07 \\
\hline$N E W 6$ & 3.75 & 0.07 \\
\hline$B G^{2}$ & 3.50 & 0.06 \\
\hline$N E W 4$ & 2.33 & 0.04 \\
\hline Gry E̊Ang6 & 2.01 & 0.04 \\
\hline Swan 1 & 0.33 & 0.01 \\
\hline CछฐV6 & 0.00 & 0.00 \\
\hline ConE์Den4 & 0.00 & 0.00 \\
\hline Gry $\Xi^{2} A n g 2$ & 0.00 & 0.00 \\
\hline Grey $\Xi^{2} A n g 4$ & 0.00 & 0.00 \\
\hline$C \mathcal{E}^{2} V 8$ & 0.00 & 0.00 \\
\hline Caer2 & 0.00 & 0.00 \\
\hline Nport1 & 0.00 & 0.00 \\
\hline Bridge 1 & 0.00 & 0.00 \\
\hline Bridge2 & 0.00 & 0.00 \\
\hline$N E W 5$ & 0.00 & 0.00 \\
\hline Gry E̊Ang3 & 0.00 & 0.00 \\
\hline$N E W 7$ & 0.00 & 0.00 \\
\hline$N E W 9$ & 0.00 & 0.00 \\
\hline Pow1 & 0.00 & 0.00 \\
\hline Pow2 & 0.00 & 0.00 \\
\hline Pow4 & 0.00 & 0.00 \\
\hline Cध̊V9 & 0.00 & 0.00 \\
\hline$C E^{2} V_{1}$ & 0.00 & 0.00 \\
\hline Carm2 & 0.00 & 0.00 \\
\hline Carms & 0.00 & 0.00 \\
\hline$N E W_{1}$ & 0.00 & 0.00 \\
\hline$W W_{1}$ & 0.00 & 0.00 \\
\hline NPT4 & 0.00 & 0.00 \\
\hline$C \mathcal{E}^{2} V_{10}$ & 0.00 & 0.00 \\
\hline NPT3 & 0.00 & 0.00 \\
\hline
\end{tabular}

As Table 5.4 illustrates, 25 individuals have a betweenness centrality score of 0 , which suggests that they are not acting as "bridging" or "connecting" agents in their role. Caer3 (the light green square towards the bottom left of the sociogram) has the 
highest betweenness score of 432.97 which indicates that this Caseworker is positioned on more than four hundred of the shortest paths between pairs of unconnected Caseworkers and hence has the potential to link more than four hundred pairs of unconnected Caseworkers together. As such they can be considered to hold a pivotal "bridging" position in the wider Caseworker network. Similarly, 5 other Caseworkers (MT1, RCT5, RCT1, Swan3 and Carm1) have betweenness scores ranging from 293 to 356 .

In sum, what the betweenness measures shows is that certain Caseworkers can create links between colleagues within their own organisation who would otherwise not be collaborating. This can be instrumental in the flow of information or resources (including capital) around the network.

\section{Patterns of interaction within the Caseworker network}

Comparing degree centrality scores to the betweenness centrality scores as shown in Table 5.5 below, it is evident there is some overlap of those who have the highest scores. For instance, RCT5 (the large purple blue square in the middle of the sociogram) has the highest degree centrality score of all the Caseworkers $(n=19)$ and has the third highest betweenness centrality score (354.27). Similarly, Caer3 has the third highest degree centrality score $(n=17)$ and has the highest betweenness centrality score (432.97).

Table 5.5-Comparison of betweenness centrality and degree centrality scores

\begin{tabular}{l|l|l}
\hline Caseworker & \multicolumn{1}{l}{ Degree centrality } & \multicolumn{1}{l}{ Betweenness } \\
\hline RCT5 & 19 & 354.27 \\
CAER3 & 17 & 432.97 \\
MT1 & 11 & 356.45 \\
RCT1 & 7 & 324.36 \\
SWAN3 & 12 & 293.74 \\
CARM1 & 11 & 293.53 \\
\hline
\end{tabular}

Of the participants who had a betweenness score greater than 200.00, four do not feature amongst the highest degree centrality score list (these include MT1, RCT1, Swan3, and Carm1). This suggests that there are two Caseworkers who are key to this network because they are both link agents (hubs of activity) and bridging agents. These individuals are directly and highly connected but also occupy pivotal positions in the Caseworker network in that they can link colleagues who are otherwise unconnected. 
To summarise, thus far, results of the social network analysis have been used to explore the types of network connections that Caseworkers make with each other within Care \& Repair Cymru. Using the measure of degree centrality to measure the number of direct connections made between Caseworkers, it was apparent that on average most have relatively few direct connections to others. However, there are some individuals who are highly connected and so could be important to the network because they are able to reach a greater number of people, which is crucial in the spread of knowledge, skills and contacts throughout a network. Additionally, results for the measure of betweenness centrality indicate that some Caseworkers occupy more pivotal positions in the network because they act as bridges between people and facilitate contact, with the potential that these new relationships help with the diffusion of knowledge.

\section{Missing data}

There are some limitations to the analysis because there is missing data from nonresponders, which meant that the whole network was not examined. However, it is important to acknowledge the missing data or non-responders. There were 22 nonresponders and while some of these individuals have indegree scores (as indicated by other Caseworkers) their corresponding centrality measures had to be excluded from the full analysis. The reason for this exclusion was because the network data for these individuals was incomplete, and any subsequent analyses using this data would be erroneous and misleading. Appendix item 14 provides a summary of the centrality scores of the non-responders.

As illustrated in Appendix item 14, the non-responder Caseworkers have no outdegree scores because these individuals did not respond, and so there was no data available to indicate outward links made by these individuals to others. However, many of these individuals were identified by others as being connections within the larger Caseworker network. Consequently, it can be determined that there are several prestigious actors within the Caseworker network who have not been included. For instance, $\mathrm{C} \& \mathrm{~V} 4$ has a high indegree score of 8 which is amongst the highest in the network overall, however this individual had to be excluded from the larger analysis because they did not respond to the Caseworker questionnaire and to include this data in the analysis would lead to erroneous and invalid results. 


\section{Caseworkers networking as cliques}

The next part of the analyses aimed to address the research question about whether the Caseworker network might contain sub-groups or cliques $(\mathrm{Q} 1 \mathrm{~b})$.

The structure of the Caseworker network was analysed to see if there was evidence of smaller sub-groups or 'cliques' which may appear within the larger network. A 'clique' can be understood as small groups of at least three individuals contained within a larger network that all connect to each other. They illustrate how larger networks (in this instance the Care \& Repair Cymru Caseworker network) can be comprised of smaller networks (see Chapter Four).

Table 5.6 shows the results of 'clique' analysis (i.e. the numbers and sizes of the subgroups within the larger network).

Table 5.6- Illustration of the numbers of clique sizes found in the Caseworker network

\begin{tabular}{|c|c|c|}
\hline Clique size & Number identified (n) & $\begin{array}{l}\text { Number of same agency } \\
\text { cliques (n) }\end{array}$ \\
\hline 3 & 27 & 6 \\
\hline 4 & 24 & 6 \\
\hline 5 & 4 & O \\
\hline 6 & 1 & O \\
\hline $\begin{array}{r}\text { Total number of } \\
\text { cliques }\end{array}$ & 56 & 12 \\
\hline
\end{tabular}

A total of 56 different cliques with a minimum size of 3 individuals were identified within the larger Caseworker network; almost half $(n=27)$ were cliques containing three people, and another 24 contained 4 Caseworkers (Table 5.6). The larger cliques consisting of 5-6 Caseworkers were the minority with only 1 containing 6 Caseworkers. This indicates that most individuals tend to be networking in smaller (of 3 or 4 Caseworkers) rather than larger groups. Overall, these results indicate that Caseworkers tended to network and operate as smaller sub-groups, which are not just defined by agency location or membership, because 44 of the 56 cliques involve Caseworkers engaging with colleagues from different agencies.

The larger cliques consisting of 5 or 6 individuals were plotted on sociograms for the purposes of analysis. Figures 5.2-5.6 show that some Caseworkers feature in a greater number of cliques and these are highlighted in red squares. For instance, four individuals (WW7, Gwy\&Ang6, Nport4 and RCT5) feature in 3 different cliques comprising 5 individuals (see figures 5.2-5.5), as well as being members of the largest of all cliques comprising 6 Caseworkers (see figure 5.6). 
Figure 5.2- Illustration of Clique size 5 (1)

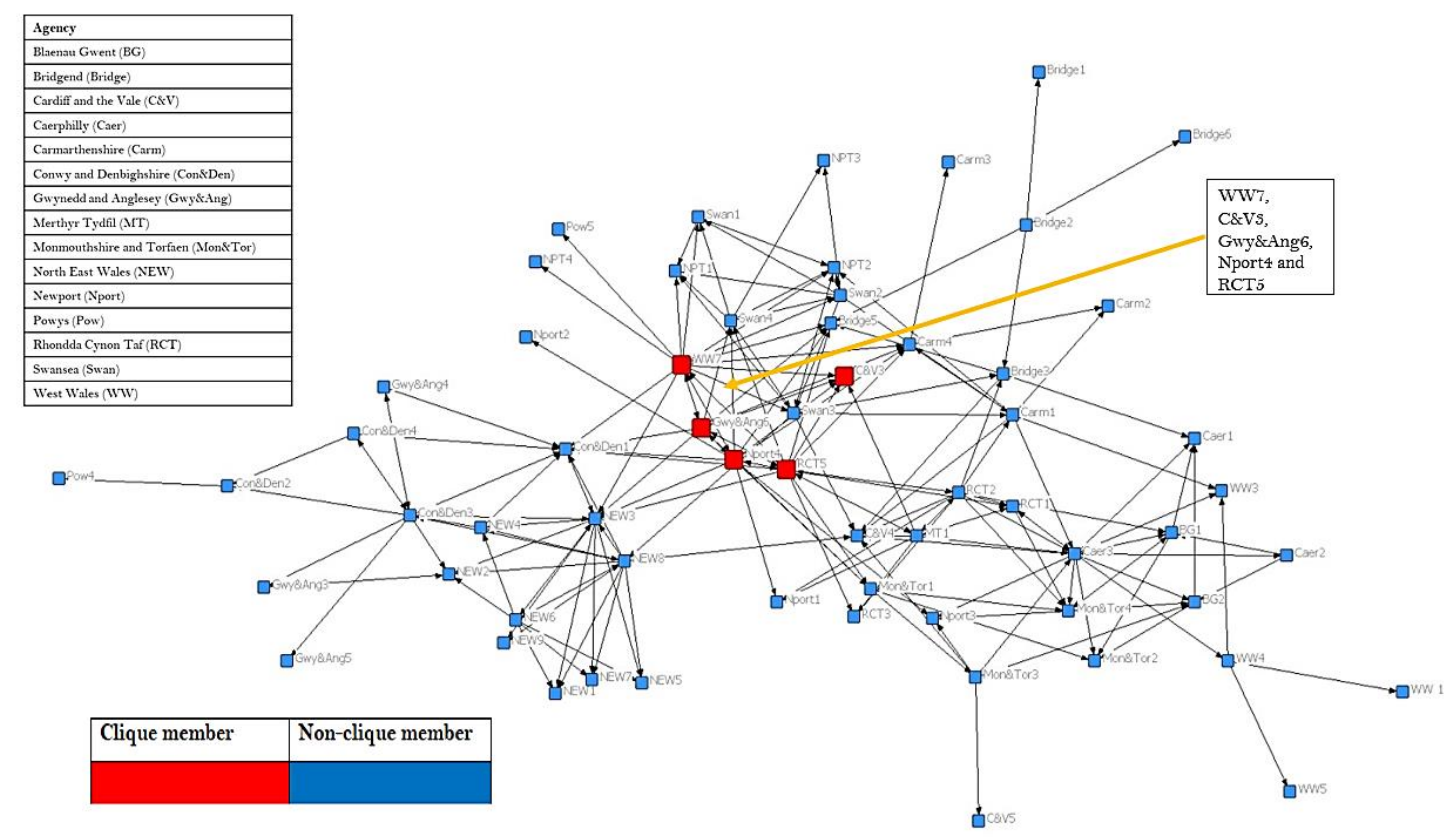

Figure 5.3- Illustration of Clique size 5 (2)

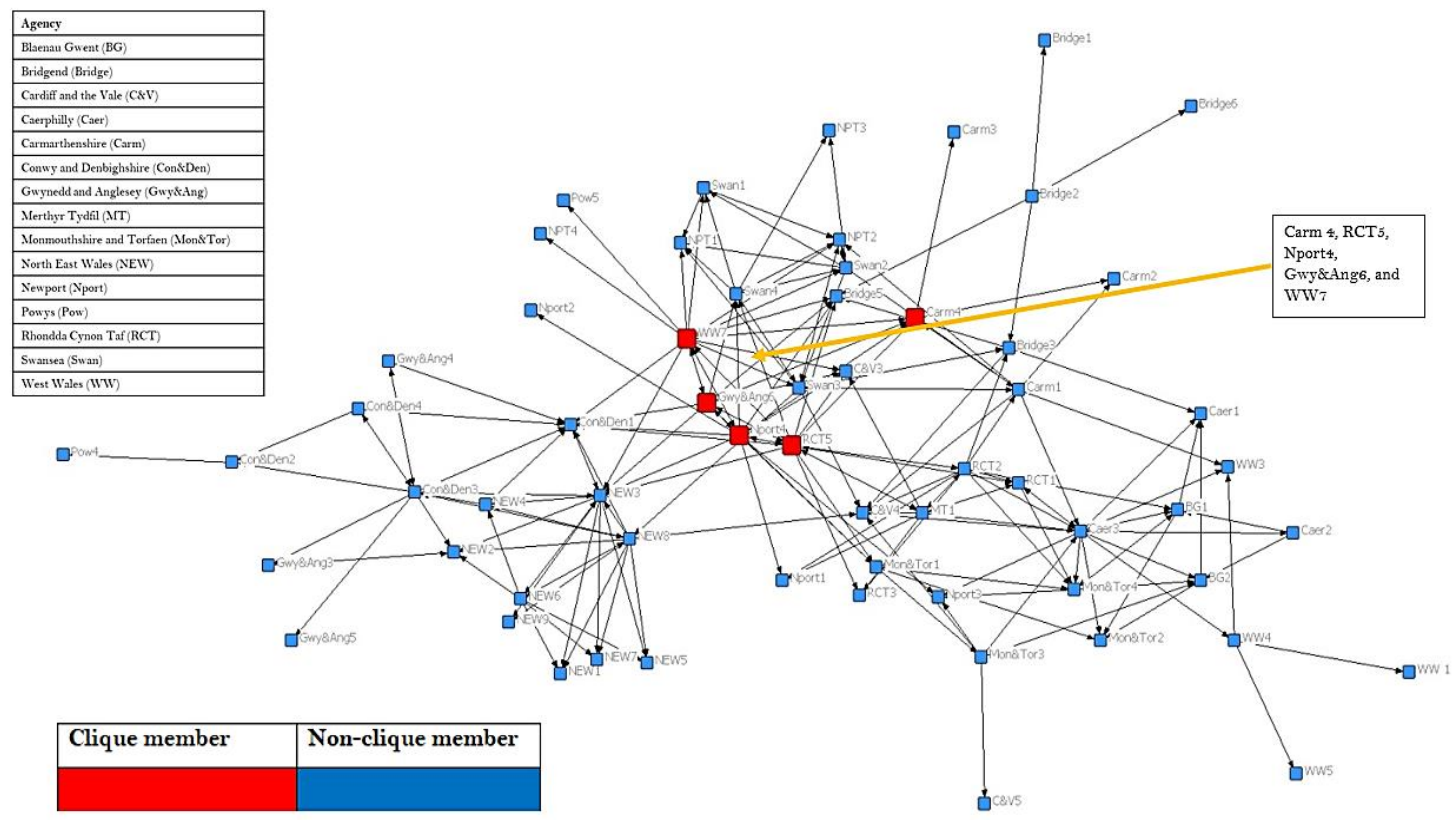


Figure 5.4- Illustration of Clique size $5(3)^{23}$

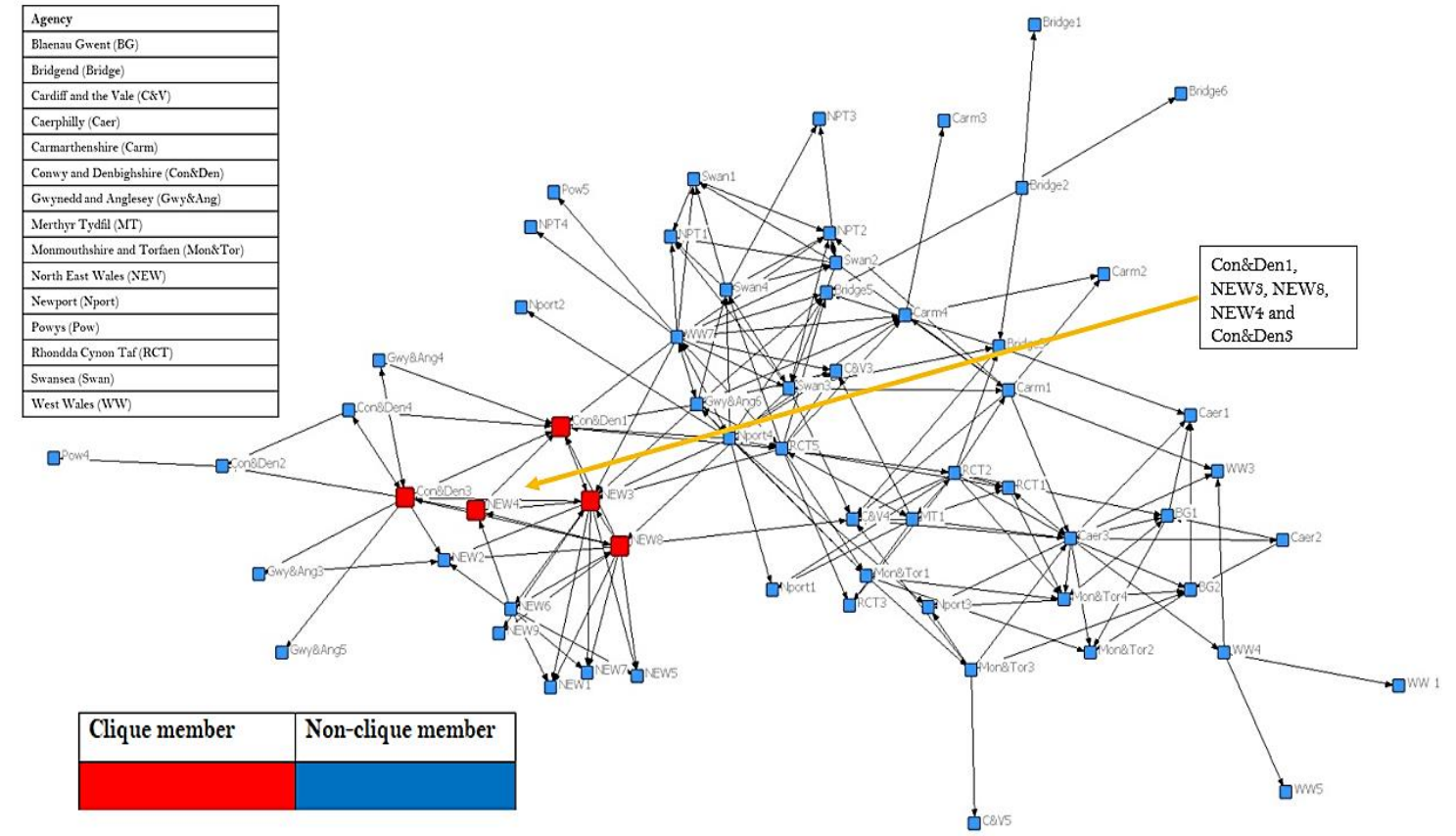

Figure 5.5- Illustration of Clique size 5 (4)

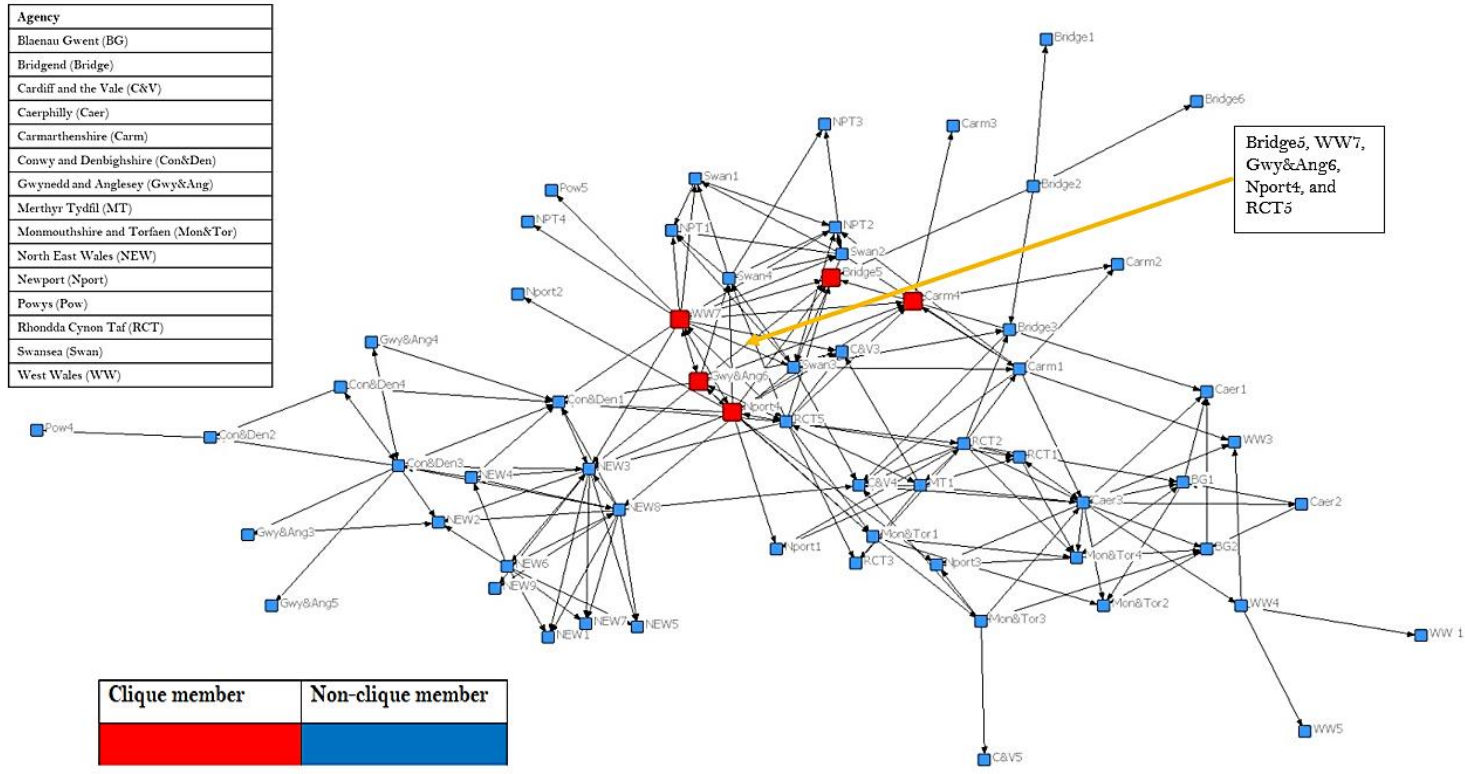

${ }^{23}$ All of the sociograms referenced are the same $\mathrm{CW}$ to $\mathrm{CW}$ network. The red boxes illustrate the clique members, and the blue are the non-clique members of the larger cliques. Group membership is not mutually exclusive. 
Figure 5.6- Illustration of Clique size 6

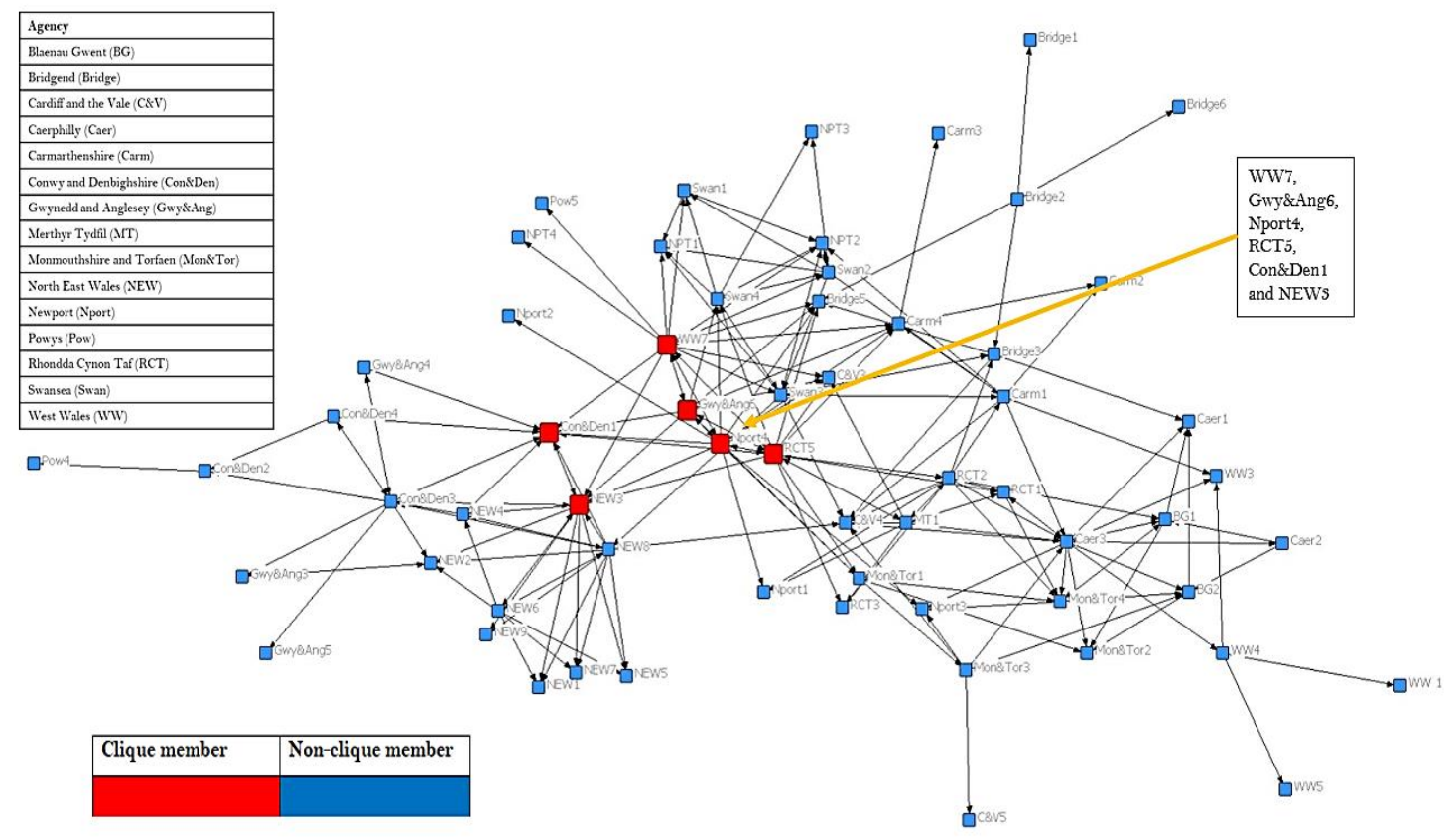

As the "clique" analysis has shown, of the 56 cliques identified, 44 were not located in the same geographic area, which again suggests that there is inter-agency working and collaboration in the delivery of services to older people throughout Wales. However, the clique analysis has also shown that the right-hand side of the sociogram does not contain any of the larger subgroups.

This suggests that other factors are at play in influencing the formation of subgroups and inter-agency working. For instance, the existence of Caseworker subgroups could reflect the need for them to build links with colleagues in other agencies with particular skills, expertise or specialisms, in other words, they are seeking to complete their work by accessing different capitals across the Caseworker network. Further statistical analysis is presented later in this chapter, which explores whether Caseworkers' capitals are associated with the different patterns of interaction observed, but qualitative feedback from Chief Officers does suggest this to be the case.

A South Wales Chief Officer spoke about the specialisms within the Caseworker roles and how this has influenced their collaboration and networking.

'...we've got 2 Caseworkers who do pretty much all the works around affordable warmth, so look at boiler replacement, window, doors etc...but because of their specialist knowledge they'll probably cover both counties as, as a team yet they would work with their colleagues across the Caseworker service and support them' [CO4].

Here, Caseworkers are linking with each other across counties (i.e. agencies), and hence maximising use of the specific social and human capital resources they have at 
their disposal. This quote also highlights the importance of Caseworkers building and maintaining good relationships with each other to create supportive collaborations and facilitating the transmission of this expertise and knowledge throughout the Caseworker network. This was also discussed by the Caseworkers in their focus group, in which it was acknowledged that building supportive relationships was an important way of finding and delivering solutions to clients:

... to source information from each other as caseworkers at events like this [networking meeting], team meetings in our offices just on a day to day basis, um discussing problems and discussing ways forward... with regards to Care E Repair as an organisation, there is a massive knowledge of support... as I said you know we can just pick up the phone if we need more information on Hardship funding we can discuss it with them' [Caseworker in focus group].

Another Chief Officer also alluded to how inter-agency connectivity (as demonstrated through the "cliques" results) can be shaped by the geographic region in which the agency is based.

'It comes down to relationships I think and the motivation of one party or all parties to deliver and... because of the physical geography of [Mid Wales] we will often... use colleagues from bordering agencies to deliver some of our rapid response work because it's actually quicker and cheaper... '[CO13].

This quote offers a further explanatory insight into the role that other factors beyond individual Caseworker attributes, (in this instance, the physical geography of Wales and cost and efficiency considerations) can play. From a qualitative perspective, geography, cost and time also appear to be contextual factors underpinning interagency networking, alongside individual, social and human capital assets.

\section{Types of Caseworker connections: patterns of networking}

Building upon these results, and as way of trying to develop more understanding of the underlying characteristics of the Caseworker network, a further stage of statistical analysis was undertaken. This time the data was analysed in terms of distinct networking profiles $\left(\mathrm{Q}^{2} \mathrm{a}\right)$, to examine if the way Caseworkers were connecting could by typified or grouped together. This approach was informed by previous research (Stephenson, 2005; Woodcraft, 2011), which identified different networking characters or patterns within social networks (see Chapter Two).

For this purpose, exploratory latent profile analysis was undertaken. Exploratory models were developed to establish whether the Caseworkers' indegree and outdegree scores (two dimensions of how Caseworkers were connecting with each other) could be grouped together as distinct classes (see Chapter Four). Results show that two statistically significant classes or two distinct patterns of networking can be identified in the networking data (see Table 5.7). 
Table 5.7 -Latent profile analysis using MPlus

\begin{tabular}{|c|c|c|c|c|c|c|c|}
\hline $\begin{array}{l}\text { Number } \\
\text { of } \\
\text { classes } \\
\text { included } \\
\text { in model }\end{array}$ & $\begin{array}{l}\text { Akaike } \\
\text { (AIC) }\end{array}$ & $\begin{array}{l}\text { Bayesia } \\
\text { n (BIC) }\end{array}$ & $\begin{array}{l}\text { Adjusted } \\
\text { Bayesian } \\
\text { (aBIC) }\end{array}$ & Entropy & $\begin{array}{l}\text { Vuong- } \\
\text { Lo- } \\
\text { Mendell- } \\
\text { Rubin } \\
\text { ratio test, } \\
\text { log- } \\
\text { likelihood } \\
\text { of } \mathrm{H}_{\text {。 }}\end{array}$ & $\begin{array}{l}\text { Vuong-Lo- } \\
\text { Mendell- } \\
\text { Rubin } \\
\text { adjusted } \\
\text { LRT test }\end{array}$ & $\begin{array}{l}\text { P-Value } \\
\text { Vuong- } \\
\text { Lo- } \\
\text { Mendell } \\
\text {-Rubin } \\
\text { ratio } \\
\text { test }\end{array}$ \\
\hline 2 classes & -417.59 & -403.54 & -425.53 & 0.91 & 200.23 & 28.62 & 0.04 \\
\hline 3 classes & -420.54 & -400.47 & -431.89 & 0.92 & 215.79 & 8.26 & 0.17 \\
\hline
\end{tabular}

Table 5.7 illustrates that 2 classes and 3 classes (distinct patterns of networking) can be found within the data, however the analysis was ceased after conducting a 4-class model analysis because the fit indices and p-value did not dramatically improve as the class size increased (see Chapter Four). Based on an examination of the output values and the p-values, the 2-class model fits the data better. The P-Value for the 2-class model is .0041 (less than 0.05), which is not improved when applying the 3-class model to the data (the p-value increases to become more than 0.05 (0.17)). Table 5.8 also indicates that the entropy does improve in the 3-class model (0.92) compared to the 2-class model (0.91). The measure of entropy does remain acceptable which reaffirms that the 2 -class model is a better fit to the Caseworkers' network data.

In sum, the latent profile analysis shows that there are 2 statistically viable classes or patterns of networking, which capture how Caseworkers are networking.

Table 5.8- Observed characteristics of the most probable class membership

\begin{tabular}{|c|c|c|c|c|c|c|c|}
\hline $\begin{array}{l}\text { Clas } \\
\mathrm{s}\end{array}$ & $\begin{array}{l}\text { Number } \\
\text { of } \\
\text { individua } \\
\text { ls in the } \\
\text { class }\end{array}$ & $\begin{array}{l}\text { Min } \\
\text { Indegre } \\
\text { e score }\end{array}$ & $\begin{array}{l}\text { Max } \\
\text { Indegre } \\
\text { e score }\end{array}$ & $\begin{array}{l}\text { Mean } \\
\text { Indegre } \\
\text { e score } \\
\text { (SD) }\end{array}$ & $\begin{array}{l}\text { Min } \\
\text { Outdegr } \\
\text { ee score } \\
\text { (SD) }\end{array}$ & $\begin{array}{l}\text { Max } \\
\text { Outdegr } \\
\text { ee score }\end{array}$ & $\begin{array}{l}\text { Mean } \\
\text { Outdegr } \\
\text { ee score }\end{array}$ \\
\hline 1 & 42 & 2 & 8 & $\begin{array}{l}4.08 \\
(1.80)\end{array}$ & 6 & 15 & $\begin{array}{l}10.08 \\
(2.78)\end{array}$ \\
\hline 2 & 13 & 0 & 10 & $\begin{array}{l}2.24 \\
(2.07)\end{array}$ & 0 & 6 & $1.52(1.80)$ \\
\hline
\end{tabular}

Once the number of groups within the patterns of networking were confirmed using LPA, the analysis progressed to determine which group each Caseworker is most likely to belong to based on their indegree and outdegree scores. This data was then explored in SPSS using descriptive statistics to explore some of these observed characteristics of these groups (see Table 5.8). 
Based on probable class membership and the means scores of indegree and outdegree, Group 1 has greater indegree and outdegree scores compared to Group 2. Taking the indegree and outdegree scores into consideration the groups can be labelled according to their centrality scores. Group 1 who have high scores of indegree and outdegree (indicating a high number of links) is the 'high connectivity group' and Group 2 with low scores of indegree and outdegree (indicating a low number of links) is the low connectivity group ${ }^{22}$.

The latent profile analysis indicated that there are two statistically viable groups or patterns of networking between Caseworkers can be found within the data. These groups reflect two different modus operandi in how the Caseworkers network together. One group represents 'high level connectors' (high number of links) and the other indicates 'low level connectors' (low number of links).

\section{Explaining differences in connectivity in the Caseworker network}

Thus far, statistical analysis has been used to try and understand how Caseworkers are connecting with each other using: social network analysis mapping and developing measures, which show how they make and receive links with colleagues (degree centrality); how they act as bridging agents by connecting other Caseworkers who would otherwise not be in contact $(\mathrm{Q} 1 \mathrm{a})$; exploring if Caseworkers were networking as sub-groups $(\mathrm{Q} 1 \mathrm{~b})$; and whether there were distinct patterns of interaction amongst Caseworkers that could be typified (Q2a). This section will use the results from the analysis to offer explanations as to why Caseworkers are connecting in these different ways by drawing on data collected about their capital attributes and contextual factors $\left(\mathrm{Q}^{2} \mathrm{~b}\right)$.

Statistical analyses (See Chapter Four) was undertaken to establish if any of the social or human capital (e.g. previous work experience), or socio-demographic (age) characteristics and other contextual factors (issues on the ground) (see table 5.1) were associated with differences in the Caseworkers' individual outdegree and indegree scores in the Caseworker network. Analyses on association between independent variables (capital, contextual and socio-demographic) and Caseworkers' scores of indegree and outdegree were run separately.

${ }^{24}$ See Appendix item 15- for technical explanation of how the patterns of networking were labelled and explored. 
Results indicate that two factors showed statistically significant associations with differences in Caseworkers' indegree and outdegree scores ${ }^{25}$ or the Caseworkers' patterns of interaction. These were the length of time a Caseworker has been in post, and whether they had previous experience of working in the business job sector.

Having previous work experience in the business job sector was associated with differences in individual indegree scores $(\mathrm{F}(1,53)=5.28, \mathrm{p}<.05)$. Caseworkers who indicated that they had previously worked in the business sector (e.g., such as banking and finance, business, legal, marketing media and PR, recruitment and HR) had a greater mean normalised indegree score (0.05), compared to those who have not had this type of work experience (0.03). More simply those who have worked in the business sector have higher indegree scores.

The other factor that was significantly associated with differences in individual measures of centrality scores was the length of time in role, which was associated with differences in individual outdegree scores $(\mathrm{F}(3,51)=3.08, \mathrm{p}<.05)$.

Caseworkers who have been in post for the least amount of time had the greatest outdegree scores $\mathrm{m}=0.90(\mathrm{SD}=0.79)$. Yet, those who have been in the role between 13 years have the lowest outdegree scores $\mathrm{m}=0.26(\mathrm{SD}=0.38)$. Furthermore, those who had been in the post $5+$ years also had a significantly lower outdegree score than Caseworkers that had been in post 6 months to a year $(p=.042)$. Similarly, those who had been in post 1-3 years also had significantly lower outdegree scores than Caseworkers who had been in post 6 months to 1 year $(p=.046)$.

In sum, the differences in the Caseworkers' individual degree centrality scores are associated with two different variables: having previous experience in the business job sector and length of time in role. Greater scores are associated with the length of time that Caseworkers have been in post and having previous work experience in the business job sector.

${ }_{25}$ Other factors examined included : Agency Location, Gender, Age group Finance and Management vocational qualification, Health and Social Care vocational qualification, IT vocational qualification, Energy vocational qualification, Practical vocational qualification, NVQ vocational qualification, Health and Safety training course, Housing and Energy training course, Finance training course, Administrative and Clerical skills training course, Interpersonal skills training course, previous job sector-Public service healthcare, previous job sector- Public Service, previous job sector-Business, previous job sectorPractical/Technical, previous job sector-Charity and Voluntary, previous job sectorCustomer Services, previous occupational role- Managerial, previous occupational roleAdministrative, previous occupational role-Customer Service, previous occupational rolePractical, job title, funding, length of time in role and number of training courses attended. 


\section{Factors close to explaining numbers of Caseworker connections}

There were several factors which were close to showing a statistically significant association with the indegree and outdegree scores. Although no statistically significant inference can be drawn from these results, they are mentioned here because they may provide some useful insights to inform analysis of indegree and outdegree scores in a larger study.

There is a weak association between indegree and agency location $(\mathrm{F}(15,39)=1.82$, $\mathrm{p}=.07)$, indicating there may be some regional variations in the indegree scores that Caseworkers have. While there are observed differences in indegree scores by agency location as shown in Figure 5.1, the differences are not statistically significant. For example, Caseworkers in Conwy and Denbighshire have the greatest mean indegree score $(\mathrm{M}=0.06, \mathrm{SD}=0.05)$ compared to Caseworkers in Cardiff and the Vale $(\mathrm{M}=$ $0.00, \mathrm{SD}=0.00)$. If the study population of Caseworkers were bigger, regional variation may be statistically significant in explaining different indegree scores of Caseworkers. Linking this result back to one of the Chief Officer extracts which alluded to specialist knowledge that certain Caseworkers develop, regional variations of indegree scores may reflect different concentrations of capital that others wish to access.

Having previous work experience in the practical or technical sector is also weakly associated with differences in the mean indegree scores of Caseworkers (F(15, $39)=3.56, p=.05)$. The Caseworkers with experience of working in practical or technical roles, including construction and engineering, creative arts and design, environment and agriculture, IT and technology sectors have a lower mean indegree score $(\mathrm{M}=0.02, \mathrm{SD}=0.02)$ than those who have not worked in this area $(\mathrm{M}=0.04, \mathrm{SD}=$ $0.03)$.

Caseworkers who have previously worked in technical roles may have built up forms of capital which is not wholly applicable to the Caseworker role compared to other forms of capital, which may influence how Caseworkers are connecting with their colleagues. This seems logical because the Caseworker role overall has some technical knowledge, but most expertise and technical queries are often directed to Technical Officers who have a more technically orientated role. 
To summarise, if the study population were larger two factors may be statistically significant in explaining the indegree scores of Caseworkers. These include agency location and having previous work experience in practical or technical roles.

\section{Factors which are associated with patterns of networking}

The final stage to this part of the analysis examined if probable group membership to the high connectivity group (group 1) or the low connectivity group (group 2 ), identified through the exploratory latent profile analysis results, was associated with any of the independent variables captured from the questionnaire.

The results of the Chi-square test of independence provides evidence that there is a relationship between probable group membership and Caseworkers having previous work experience in the customer service sector $\mathrm{X}^{2}(1, \mathrm{~N}=55)=5.28, \mathrm{P}<.05$. While the low connectivity group (group 2) contains less Caseworkers $(\mathrm{n}=13$ ), almost two thirds $(59.5 \%)$ of this group had previous work experience in the customer service sector. On the other hand, over three quarters of the high connectivity group did not have previous experience of working in the customer service sector (76.9\%). This indicates that previous work experience in the customer service sector is associated with possible group membership to the low connectivity group.

No other independent variables were associated with different group membership or patterns of networking.

\section{Chapter summary}

This chapter has presented the results of the social network analysis, which has addressed four of the research questions posed in Chapter Two: literature review.

First the results which examined the types of connections Caseworkers make with each other across the Caseworker network (Q1a) were explored. The results present an impression of how the Caseworkers are networking and linking with their colleagues to deliver services to older people. The first main finding was that Caseworkers are connecting with each other in different manners i.e. there are some Caseworkers who link more with their colleagues than others, some receive more links from others and finally some Caseworkers act as bridging agents by connecting otherwise unconnected colleagues.

The second part of the social network analysis examined if Caseworkers were networking as smaller groups within the larger Caseworker network (Q1b). The results indicated that Caseworkers network with each other as sub-groups. Mainly 
Caseworkers network as small groups, but there are some larger sub-groups containing five or six individuals. These groups are not geographically constrained, which indicates that there must be some other factor which influences how Caseworkers network within the Care \& Repair Cymru network (i.e. social or human capital).

Subsequent statistical analyses aimed to determine patterns of interactions between Caseworkers (Q2a). The results revealed that the networking activities of Caseworkers can be grouped together based on their indegree and outdegree score. These patterns of networking include high connectors and low connectors.

Finally, this chapter examined if the different patterns of interaction and the indegree and outdegree scores were associated with any of the capital, contextual or sociodemographic factors captured in the Caseworker questionnaire. The ANOVA analyses concluded that indegree scores were associated with having previous work experience in the business sector, while outdegree scores were associated with the length of time a Caseworker has been in post. Furthermore, the low connectivity group was associated with previous experience of working in a practical or technical role.

This chapter explored the links that Caseworkers make with each other and the subsequent chapter explores the links that Caseworkers make with organisations outside of Care \& Repair Cymru agencies. 


\section{CHAPTER SIX}

\section{Presentation of Findings- Part Two: How Caseworkers Link with External Services}

As part of their role, Caseworkers link with organisations outside of the Care \& Repair Cymru agencies and act as service brokers for clients. This understanding has been informed by the Caseworker job description and social network literature, however, to explore how they deliver services to clients, this thesis aimed to explore the types of connections that Caseworkers make with external services. This chapter will first discuss the importance of collaborative working from the perspective of Care \& Repair Cymru staff. Second the findings from the analyses which examined the types of links (frequency and breadth) that Caseworkers make with external organisations (Q3a) will be presented. Finally, if any personal characteristic or contextual factors are associated with these links $(\mathrm{Q} 3 \mathrm{~b})$ is explored.

\section{The importance of inter-organisational working}

Several Chief Officers addressed the importance of collaborative working in their face-to-face interviews. For example; one South Wales Chief Officer stated that '...partnerships are vital to the success of the agencies.' (CO8). This notion was explored in greater detail by another Chief Officer who alluded to factors external to the Caseworker role e.g. saving money and economies of scale, as reasons for supporting collaborative working;

'...rather than duplicate what other people do, we will refer on and work in partnership with people...' [CO 10].

These quotes not only highlight that Care \& Repair Cymru agencies already understand the importance of collaborative working, but they are already working in collaboration with external organisations to facilitate independent living and deliver services to older people throughout Wales.

To examine in more depth, the nature of these collaborative links, the following section provides results of statistical analysis which has looked at the frequency, breadth and type of connections that Caseworkers are making with external organisations. This analysis was initially informed by responses which Caseworkers gave during the January 2015 workshop, and which fed into the development of questions included in the online self-completion questionnaire (see Appendix item 3). 
This enabled the case workers to indicate both the types of organisations they linked with as part of their work, as well as how often they did this (daily, weekly, monthly, one off and no contact).

\section{The organisations that Caseworkers connect with}

In the first instance, the types of organisations that Caseworkers link with were explored. The findings from the questionnaire indicated that Caseworkers link with 383 different organisations across 16 different types of organisations which are grouped in Table 6.1 under 6 sectors (financial assistance, fundraising, referrals, domestic help, digital inclusion, healthy homes check and home maintenance). The frequency of these links are summarised in Table 6.1. 
Table 6.1- Frequency (\%) of contact with external organisations $(n=55)$

\begin{tabular}{|c|c|c|c|c|c|}
\hline $\begin{array}{l}\text { Type of } \\
\text { organisation }\end{array}$ & $\begin{array}{l}\text { Daily } \\
\text { contact }\end{array}$ & $\begin{array}{l}\text { Weekly } \\
\text { contact }\end{array}$ & Monthly & $\begin{array}{l}\text { One-off } \\
\text { contact }\end{array}$ & $\begin{array}{l}\text { No- } \\
\text { contact }\end{array}$ \\
\hline $\begin{array}{l}\text { Financial } \\
\text { Assistance }\end{array}$ & 14.5 & 49.1 & 21.8 & 7.3 & $7.3^{26}$ \\
\hline $\begin{array}{l}\text { Fundraising- } \\
\text { Businesses }\end{array}$ & $\mathrm{O}$ & 32.7 & 40.0 & 21.8 & 5.5 \\
\hline $\begin{array}{l}\text { Fundraising- } \\
\text { Public Sector }\end{array}$ & 29.1 & 25.5 & 29.1 & 10.9 & 5.5 \\
\hline $\begin{array}{l}\text { Fundraising- } \\
\text { Charities }\end{array}$ & 3.6 & 14.5 & 43.6 & 32.7 & 5.5 \\
\hline $\begin{array}{l}\text { Fundraising- } \\
\text { Professional } \\
\text { Occupations }\end{array}$ & 1.8 & 20.0 & 43.6 & 25.5 & 9.1 \\
\hline $\begin{array}{l}\text { Referral- } \\
\text { Personal } \\
\text { Support }\end{array}$ & 1.8 & 18.2 & 36.4 & 32.7 & 10.9 \\
\hline $\begin{array}{l}\text { Referral- Re- } \\
\text { enablement }\end{array}$ & 23.6 & 34.5 & 25.5 & 10.9 & 5.5 \\
\hline Referral- Health & 21.8 & 40.0 & 18.2 & 14.5 & 5.5 \\
\hline $\begin{array}{l}\text { Referral- } \\
\text { Personal Care }\end{array}$ & 7.3 & 23.6 & 30.9 & 23.6 & 14.5 \\
\hline Domestic Help & 1.8 & 12.7 & 25.5 & 25.5 & 34.5 \\
\hline Digital Inclusion & 1.8 & 29.1 & 36.4 & 25.5 & 7.3 \\
\hline $\begin{array}{l}\text { Healthy Homes } \\
\text { Check-Fuel } \\
\text { Poverty }\end{array}$ & 3.6 & 27.3 & 45.5 & 18.2 & 5.5 \\
\hline $\begin{array}{l}\text { Healthy Homes } \\
\text { Check-Fuel } \\
\text { Safety }\end{array}$ & 5.5 & 40.0 & 32.7 & 12.7 & 9.1 \\
\hline $\begin{array}{l}\text { Healthy Homes } \\
\text { Check-Slips, } \\
\text { Trips and Falls }\end{array}$ & 9.1 & 40.0 & 30.9 & 16.4 & 3.6 \\
\hline $\begin{array}{l}\text { Healthy Homes } \\
\text { Check-Security } \\
\text { of the home }\end{array}$ & 0 & 1.8 & 14.5 & 52.7 & 30.9 \\
\hline $\begin{array}{l}\text { Home } \\
\text { maintenance }\end{array}$ & 40.0 & 36.4 & 7.3 & 10.9 & 5.5 \\
\hline
\end{tabular}

\section{How frequently do Caseworkers link with these organisations?}

On a daily basis, and as would be expected, Caseworkers are the most likely to be linking with organisations dealing with home maintenance (40.0\%); the next most prevalent type of daily connections they make are with 'public sector' organisations for the purposes of fundraising (29.1\%); but they do not report spending any time each day contacting 'businesses' (for fundraising) or 'security' organisations (as part of the healthy homes check).

${ }^{26}$ The rows in this Table add up to $100 \%$ 
Each week, almost half $(49.1 \%)$ of the Caseworkers make contact with financial assistance organisations. Furthermore two-fifths (40.0\%) indicated that they made referrals to 'health' organisations, and as part of their health homes check organisations, the same proportion were in touch with 'fuel safety' and 'slips, trips and falls' organisations.

From the data, it appears that between 40-46\% make monthly connections with 'fuel poverty' (45.5\%) organisations, as well as links to fundraising bodies through 'charities' and 'professional/ occupational' organisations (43.6\%).

Clearly there are some service domains which the Caseworkers are less likely to link with on behalf of their clients; for example over $60 \%$ have only one off (25.5\%) or no $(34.5 \%)$ contacts with domestic help organisations; and the majority - over $80 \%$ have only one off $(52.7 \%)$ or no $(39.9 \%)$ contact with security services which form part of the healthy homes check. So, in both cases, around one-third of the Caseworkers have no contact or make no referrals to domestic help and security organisations.

In sum, the frequency with which Caseworkers are linking with external organisations varies considerably, depending on whether their work involves fundraising, referral, health home check activities, home maintenance, domestic help or digital inclusion services. For instance, in terms of frequency Caseworkers appear to spend a lot of time on fundraising activities. At least two-thirds of participants reported linking with fundraising organisations on a monthly or more frequent basis. Additionally, frequent referrals to organisations are focused around er-enablement and health services; and in terms of helping to create healthy living environments, it is only links with security services that are less likely to be engaged with as around a third of all the Caseworkers indicated that they have no contact with these types of organisations. Finally, linking with domestic help services is not something that the majority of the Caseworkers are involved with. There may be several explanations for this, including the needs of clients and the condition of the housing stock and the may influence contact. This will be discussed in greater depth in Chapter Eight: Discussion and Limitations.

\section{What factors shape the frequency of contact with these organisations?}

Having established the types of organisations and frequency with which Caseworkers link with organisations external to the Care \& Repair Cymru agencies, the next 
question is to examine which factors (individual and contextual) are associated with different frequencies of connections made by Caseworkers with external organisations (Q3b). Several capital and contextual variables were used in the analyses (See Chapter Four) because previous social network studies and capital theories (reviewed in Chapter Two) indicated that these factors may help explain the different forms of working relationships that Caseworkers build and utilise to deliver services to their clients.

Specifically, previous work roles and experiences, length of time in the role, issues faced by Caseworkers on the ground and agency location were tested because capital literature indicated that capitals could be built through experience including work and training (Becker, 1994; Coleman, 1988; Glaeser et al., 2002). Additionally, agency location was also included in the analyses because previous social network literature suggested that the local context can influence how services are delivered (Ennis \& West, 2010; Nicaise et al., 2012); as well as how it can shape networking activities e.g. by physically separating communities or groups and making it more difficult to network (Coleman et al., 1957, 1966; Ennis \& West, 2013).

Potential Group membership (i.e. being part of the high connectors or low connectors group) was also tested to determine if there was an association with how the Caseworkers are interacting with each other and the frequency of contact they make with external organisations. The rationale underpinning this was informed by social network analysis and capital theory. Past studies have developed typologies that captured how individuals were networking which was also linked to the types of links or capitals individuals had outside of the network (Stephenson, 2005; Woodcraft, 2011).

The following sections present results of the analysis which helped establish whether there were any statistically significant associations between Caseworkers' frequency of contact with external organisations and these capitals and contextual factors.

\section{Social and Human Capital factors and frequency of contact}

"Low" and "high" connectors: results show that the social capital (proxy) variable of being a 'high connector' or 'low connector' is not associated with differences in the frequency of links with external organisations.

Previous work experience in Managerial Roles: several factors are associated with differences in the frequency of links that Caseworkers build across three kinds of 
services. The first factor (a human capital variable) was if Caseworkers had previous work experience in managerial roles.

The first type of organisation associated with this factor were the frequency of connections to 'charities for fundraising' $\left(\mathrm{r}_{\mathrm{s}}(53)=.292, \mathrm{p}<.05\right)$. The analysis indicated a weak positive correlation suggesting that individuals who have this type of work experience make less frequent connections to the charity sector for the purposes of raising funds for their clients. The second type of organisation associated with this factor was the frequency of connections for 'personal support referrals' $\left(\mathrm{r}_{\mathrm{s}}(53)=-.266\right.$, $\mathrm{p}<.05)$. The analysis indicated a weak negative correlation coefficient between the two variables which suggests that those who have had previous work experience in managerial roles make more frequent connections with personal support organisations.

Finally, the frequency of connections with 'home maintenance' organisations was also associated with Caseworkers with previous work experience in managerial roles $\left(\mathrm{r}_{\mathrm{s}}(53)=.268, \mathrm{p}<.05\right)$. This factor showed a weak positive correlation, indicating that Caseworkers who have worked in managerial roles make less frequent connections with home maintenance organisations.

Overall, those who have previous experience of working in managerial positions make less frequent connections with charities and home maintenance organisations in addition to making more frequent connections with personal support organisations. This may reflect the skills and experience that these Caseworkers have built through their previous work experience, i.e. can manage most services effectively without the need for frequent contact with these organisations. However, Caseworkers who have this type of work experience make more frequent connections with personal support organisations. This contrasts with the overall findings which indicated that over $40 \%$ of Caseworkers made one-off or no contact with personal support organisations. However, the remaining $56.4 \%$ of participants make monthly or more frequent contact with personal support organisations. This may reflect the client's needs or funding opportunities available or may reflect a Caseworkers' ability to negotiate a client's access to personal support services which may arise out of working in a managerial role prior to working for Care \& Repair Cymru.

Previous work experience in the Public Service-Healthcare sector: another factor associated with different frequencies of connections with external organisations was 
a previous work experience variable (representing human capital). Specifically, there was an association between Caseworkers who had previously worked in public service (healthcare) sectors (e.g. within the NHS or social services) and frequency of contact with organisations.

The first type of organisations associated with this variable was the frequency of connections made to 'health referral' organisations $\left(\mathrm{r}_{\mathrm{s}}(53)=.275, \mathrm{p}<.05\right)$. The analysis indicated a weak positive correlation coefficient which suggests that the Caseworkers who have worked in the public service- healthcare sector previously made less frequent connections with health referral organisations.

The second type of service associated with this type of work experience are digital inclusion services $\left(\mathrm{r}_{\mathrm{s}}(53)=.452, \mathrm{p}<.01\right)$. Caseworkers who have worked in the public service- healthcare sector made less frequent connections with these types of services indicated by the moderate positive correlation coefficient.

The final type of service associated with this previous work experience variable were 'home maintenance services' $\left(\mathrm{r}_{\mathrm{s}}(53)=.296, \mathrm{p}<.05\right)$. From the analysis it can be determined that Caseworkers who have previously worked in the public servicehealthcare sector made less frequent connections with home maintenance services.

In summary, those who have worked in the public healthcare sector including the NHS and social services made less frequent connections with 3 types of services: (1) referrals to health services, (2) digital inclusion services and (3) home maintenance services. This may be due to the social and human capital that Caseworkers who have this type of experience have developed. This could indicate that Caseworkers may possess certain skills or have key connections in these sectors which mean that they do not have to make frequent connections to deliver services and meet their client's needs.

\section{Contextual Variables and Frequency of Contact}

Changing eligibility criteria for benefits claims: when examining the frequency of contact with external services, the spearman's rho texts indicated the contextual variable that was associated with differences in the frequencies of connections with external services was the issue of 'changes to eligibility criteria fir benefits claims' (changing eligibility). This variable captured whether the Caseworkers felt that this issue can make their job more difficult to fulfil. 
This factor was also addressed by one Caseworker in the qualitative section of the questionnaire when asked about what could be implemented to improve their working environment;

'... we need to be kept up to date with changes, benefits, LA grants etc... A list of organisations who give funding, what the funding is for and the criteria the clients has to fit. This should be set up and up-dated accordingly' [Caer3].

While the excerpt above does not directly illustrate how grant finding can impede service delivery, it does infer that this element of the case worker role appears to change frequently and therefore has the potential to influence their work on the ground. By inference, changes to benefits eligibility may well influence the frequency of connections that Caseworkers make with external organisations. Effectively, when tested as a contextual variable of 'changing eligibility', results showed that it was associated with different frequency of connections across 2 out of 16 different types of services.

The first type of service was 'referrals to personal care' services $\left(\mathrm{r}_{\mathrm{s}}(53)=-.276, \mathrm{p}<.041\right)$. Results showed a weak negative correlation coefficient which indicates that Caseworkers who experienced the issue of 'changing eligibility' made more frequent connections with personal care services on behalf of their clients.

Additionally, the frequency of connections with home maintenance services is also significantly associated with 'changing eligibility' $\left(\mathrm{r}_{\mathrm{s}}(53)=-.327, \mathrm{p}<.05\right)$. The analysis showed a weak negative correlation between the two factors, signifying that Caseworkers who experienced this issue on the ground made more frequent connections with home maintenance services.

In sum, Caseworkers who encountered the issue of changing eligibility criteria for benefit claims during their job role made more frequent connections with home maintenance and personal care services. These results indicate is that the way Caseworkers link with external organisations is shaped by the local context. As mentioned in Chapter One, the current social and policy context in Wales is one of austerity and welfare retrenchment. These contextual factors may provide an explanation as to why Caseworkers make more frequent connections with home maintenance and personal care services; in that the frequent connections represent a negotiation of access for their clients to be able to use these services i.e. providing evidence that the clients meet the eligibility criteria. This will be examined in greater depth in the discussion chapter. 


\section{The breadth of connections Caseworkers have with external organisations}

A second aspect to analysing the external links that Caseworkers develop was to examine if individual or contextual factors were associated with the breadth of connections with external organisations. This section will address the results that arose from the statistical analyses (See Chapter Four for the types of tests used) which explored associations between capitals and contextual factors and the number of external organisation connections ${ }^{27}$ made by Care \& Repair Cymru agency Caseworkers.

Once the count variables were coded the sum of connections that caseworkers have could be identified. As shown in Table 6.2, Caseworkers identified a total of 383 different organisations across the 16 different sectors. These include; 55 different financial assistance organisations and 12 different Public sector fundraising organisations they linked with.

${ }^{27}$ This is how the breadth of connections was measured and transformed into count variables; see Chapter Four for more information. 
Table 6.2 - Summary of the breadth of connections Caseworkers make with external organisations

\begin{tabular}{|c|c|c|}
\hline $\begin{array}{l}\text { Category of external } \\
\text { organisation }\end{array}$ & $\begin{array}{l}\text { Total number of } \\
\text { possible connections }\end{array}$ & $\begin{array}{l}\text { Mean score of } \\
\text { connections (SD) }\end{array}$ \\
\hline 1. Financial assistance & 55 & $6.65(\mathrm{SD}=3.92)$ \\
\hline 2. Fundraising- business & 21 & $3.02(\mathrm{SD}=1.66)$ \\
\hline Fundraising- public sector & 12 & $2.64(\mathrm{SD}=1.48)$ \\
\hline Fundraising- charity & 81 & $2.09(\mathrm{SD}=8.32)$ \\
\hline $\begin{array}{l}\text { Fundraising-professional/ } \\
\text { occupational }\end{array}$ & 19 & $5.53(\mathrm{SD}=3.37)$ \\
\hline $\begin{array}{l}\text { 3. Referrals: befriending/ } \\
\text { community support }\end{array}$ & 33 & $4.22(\mathrm{SD}=4.09$ \\
\hline Referrals: Re-enablement & 14 & $3.45(\mathrm{SD}=2.16)$ \\
\hline Referrals: Health & 27 & $4.51(\mathrm{SD}=3.11)$ \\
\hline Referrals: Personal Care & 6 & $1.20(\mathrm{SD}=0.73)$ \\
\hline Referrals: Domestic Help & 25 & $2.02(\mathrm{SD}=2.41)$ \\
\hline 4. Digital Inclusion & 6 & $2.05(\mathrm{SD}=1.22)$ \\
\hline $\begin{array}{l}\text { 5. Healthy Homes Check: } \\
\text { Fuel Poverty }\end{array}$ & 20 & $3.98(\mathrm{SD}=1.93)$ \\
\hline $\begin{array}{l}\text { Healthy Homes Check: Fuel } \\
\text { Safety }\end{array}$ & 11 & $2.25(\mathrm{SD}=1.06)$ \\
\hline $\begin{array}{l}\text { Healthy Homes Check: Slips, } \\
\text { trips and falls }\end{array}$ & 13 & $4.78(\mathrm{SD}=2.42)$ \\
\hline $\begin{array}{l}\text { Healthy Homes Check: } \\
\text { Security of the Home }\end{array}$ & 17 & $1.09(\mathrm{SD}=1.02)$ \\
\hline 6. Home maintenance & 23 & $4.44 \mathrm{SD}=2.43)$ \\
\hline $\begin{array}{l}\text { Total Number of External } \\
\text { Service Links }\end{array}$ & 383 & \\
\hline
\end{tabular}

\section{What factors are associated with breadth of connections?}

Length of time in role and the breadth of connections: this is the first factor (representing both social and human capital) was significantly associated with the breadth of connections with 6 different types of organisations and the length of time a Caseworker has been in post. The main findings have been illustrated in Table 6.3.

There are six types of service links which are significantly associated with the length of time a Caseworker has been in post, including; financial assistance, charities (fundraising), professional/ occupational (fundraising), personal support, fuel poverty and slips, trips and fall organisations.

One of the key findings from the analyses was that the Caseworkers who have been in post between 1-3 years have the highest mean score of connections with four out 
of six of the types of organisation (charities, personal support, fuel poverty and slips, trips and falls).

Additionally, of the two remaining types of service links (financial assistance and professional/ occupational organisations) Caseworkers who have been in post for 35 years have the highest mean scores of connections. On the other hand, the group with the overall lowest mean scores of connections with external services are the 'new starters' or those who have been in post from six months up to 1 year.

Furthermore, the post hoc tests indicated that the breadth of connections of some groups of Caseworkers was significantly different from others. For instance, Caseworkers who had been in post less than 1 year had significantly fewer connections with charities $(p=.029)$, personal support organisations $(p=.031)$, fuel poverty $(\mathrm{p}=.020)$ and slips trips and falls organisations $(\mathrm{p}=.042)$ compared to Caseworkers who have been in post between 1-3 years. Caseworkers who had been in post less than 1 year also had significantly fewer connections with charities $(\mathrm{p}=.009)$ and professional and occupational organisations $(\mathrm{p}=.028)$. New starters also had significantly fewer connections with financial assistance organisations $(p=.044)$ compared to Caseworkers who had been in post 3-5 years. 
Table 6.3- Summary of key findings: mean scores for association between length of time in role and breath organisations

\begin{tabular}{|c|c|c|c|c|c|}
\hline & & $\begin{array}{l}\text { Financial } \\
\text { assistance }\end{array}$ & Charities & $\begin{array}{l}\text { Professional/ } \\
\text { occupational }\end{array}$ & Personal Su \\
\hline \multicolumn{2}{|c|}{$\begin{array}{l}\text { Total number of possible } \\
\text { connections with external } \\
\text { organisation }\end{array}$} & 55 & 81 & 19 & 33 \\
\hline \multicolumn{2}{|c|}{ F-value and P-value } & $\begin{array}{l}(\mathrm{F}(3,51)=3.04, \\
\mathrm{p}=.037)\end{array}$ & $\begin{array}{l}(\mathrm{F}(3,51)=4.09 \\
\mathrm{p}=.011)\end{array}$ & $\begin{array}{l}(\mathrm{F}(3,51)=3.08, \\
\mathrm{p}=.036)\end{array}$ & $\begin{array}{l}(\mathrm{F}(3,51)=3.0 \\
\mathrm{p}=.036)\end{array}$ \\
\hline \multirow{4}{*}{$\begin{array}{l}\text { Mean score }(m) \\
\text { of connections } \\
\text { by Length of } \\
\text { time in role }\end{array}$} & $\geq 1$ year & $3.90(S D=4.17)$ & $4.50(S D=5.02)$ & $2.90(S D=3.03)$ & $1.80(S D=1.40$ \\
\hline & $1-3$ years & $8.00(\mathrm{SD}=3.28)$ & $14.67(\mathrm{SD}=11.53)$ & $5.22(\mathrm{SD}=4.09)$ & $6.89(\mathrm{SD}=7.2$ \\
\hline & $3-5$ years & $8.86^{28}(\mathrm{SD}=3.76)$ & $12.43(\mathrm{SD}=4.04)$ & $6.57(\mathrm{SD}=3.05)$ & $5.57(\mathrm{SD}=7.27$ \\
\hline & $5+$ years & $6.66(\mathrm{SD}=3.67)$ & $13.83(\mathrm{SD}=7.62)$ & $6.28(\mathrm{SD}=2.96)$ & $6.66(\mathrm{SD}=3.95$ \\
\hline
\end{tabular}

Notes: Values indicated in bold represent the Caseworkers who had the highest mean scores of connections with external or in italics indicate the Caseworkers who had to lowest mean scores of connections with external services. 
What is notable from these analyses is that simply being in the role for the greatest length of time does not necessarily equate with more connections with external organisations. However, it is evident that those who are relatively new to the organisation do have the lowest mean score of connections with several external services which at times are significantly different compared to other peer groups. This may because they have not yet had time to build up their network of contacts with external organisations. However, in some instances after being in post for over a year, Caseworkers have the highest mean score of connection with external organisations.

Agency Location and the Breadth of External connections: is the second factor associated with the breadth of connections Caseworkers make with organisations outside Care \& Repair Cymru. Table 6.4 summarises the statistically significant results that arose from the analyses. The breadth of connection with 7 out of the 16 different sectors was significantly associated with the contextual factor of agency location. These types of services include: fuel poverty, slips, trips and falls, businesses (for fundraising), personal support, health, home maintenance and digital inclusion organisations.

The North East Wales agency has the highest mean score of connections with five out of the seven external organisations. It also has the second highest mean score with the two remaining types of organisational links. Monmouthshire and Torfaen had the highest mean score of connections with digital inclusion services while Swansea has the highest mean score of connections with home maintenance services. In contrast, the Newport agency has the lowest mean score of connections with all seven types of organisation link.

Another key finding is that Blaenau Gwent has the joint highest mean score of connections with businesses (along with North East Wales) and the second highest mean score of connections with Health organisations. This illustrates that despite the size of the agency (Blaenau Gwent is one of the smaller agencies containing just two Caseworkers); they are still able to connect with a higher number of organisations compared to some of the larger agencies. 
Table 6.4- Summary of key findings: mean scores for association between agency location and breath of co

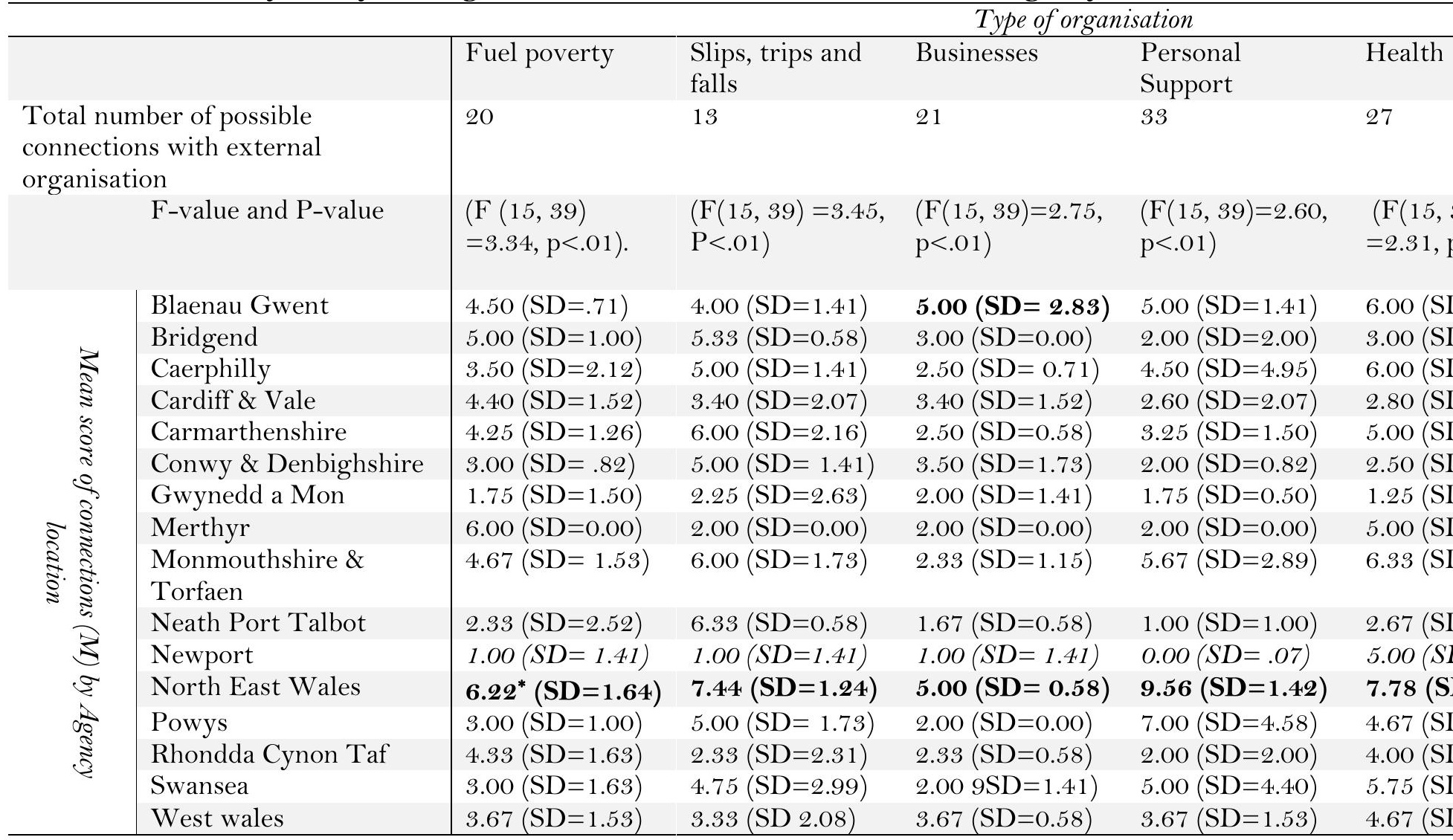

\footnotetext{
* Notes: Values indicated in bold represent the agencies which the ANOVAs indicated have the highest mean score of connec the values indicated in italics represent the agencies that have the lowest mean score of connections with external organisation some of these variables had fewer than two cases.
} 
In sum, the analyses indicate that agency location is a key factor which is associated with differences in the breadth of connections that Caseworkers have made with external organisations. Of the 15 agencies analysed, the North East Wales agency had the highest mean scores of connections with five different types of services and the second highest of the remaining two services. In contrast, Newport has the lowest mean score of connections with all but one of the different types of organisations. Merthyr has the lowest mean score of connections with digital inclusion agencies.

Post hoc tests could not be undertaken on this test because in some instances there were fewer than two cases. While this is a limitation of the small sample, the findings do indicate that Caseworkers link with different breadths of services depending on which agency they belong to. There are several possible explanations which could underpin these findings for instance, different needs of the client population, funding opportunities, different forms of accumulated social capital, contacts and experiences that the Caseworkers bring to their agency. These factors will be discussed in Chapter Eight: Discussion.

\section{Qualitative insights: Chief Officer perspectives on how the local context influences the Caseworker role}

The qualitative data gathered from the face-to-face interviews and focus groups enhances our understanding of the value placed on developing good working partnerships and how this can aid Caseworkers to deliver services to their older clients. For example, one South Wales Chief Officer stated in their interview that

'... what helps a Caseworker is the ability to access a range of services or partnerships that the agency has set up locally which enables them to find solutions as well...' $[\mathrm{CO} 2]$.

Different agency locations may well have developed different working partnerships over time or have different solutions and services available to them, funding priorities of local authorities or by the needs of the clients in specific areas e.g. health related issues.

Another explanation for the way Caseworkers were linking with external organisations will be to do with variations in job roles. For example, one mid-Wales Chief Officer commented that: 'Each agency has different job descriptions for their Caseworkers'(CO13). Variations in the breadth of connections by agency location may therefore reflect differences in the nature of the Caseworker role, which in itself, will operate differently in each local context. This was echoed by South Wales Chief Officer who commented: 
'I think with casework...you can't look at it and say you know this is what a Caseworker does... within one agency, you know with our merge with [south Wales agency]...we're only 4 miles apart but we know what our Caseworkers do is completely different to [this area agency's] Caseworkers do, which will be completely different to [other agency] to North Wales to every other agency so it is difficult to say, or define...this is a Caseworker, this is what it does' [CO10].

Another South Wales Chief Officer gave a much more restricted description of referrals:

'The Caseworker role is predominately about assisting referrals we get or clients that come our way in living independently...' [CO1].

This shows that the qualitative findings about agency location corroborate the quantitative results. Specifically, these quotes highlight that Chief Officers have different perspectives on the nature of the Caseworker role suggesting that it will vary depending on geographic location of agencies as well as job requirements, and that this in turn may shape the breadth of connections with external organisations.

\section{Relative deprivation, rurality and links with external organisations} In addition to indicating that geographical location impacts the Caseworker role and requirements, the local context also appears to influence how Caseworkers are operating to deliver services to older people. Qualitative data from Chief Officers and Technical Officers give further evidence of the significance of agency location as an influential factor in shaping the breadth of connections, particularly in relation to how contextual issues such as relative deprivation and rurality of parts of Wales can impact how Caseworkers are linking with external services.

One theme which arose during the interviews with the Chief Officer was the relative deprivation in relation to the nature of the housing stock which was encountered in different locations. For instance, when describing the local context in which their local Care \& Repair Cymru agency is operating, one mid Wales Chief Officer stated that in this region:

'...generally, we're talking about small towns, villages sparsely populated... not very good infrastructure... we are asked to help people for things like roof repairs and the sort of bigger, bigger repairs to their houses for which there are no grants available through the local authority... we often see a spike of that when the weather turns bad and the roofs start leaking...' [CO 13$]$.

The notions of rurality and how it can impact the demand on services and the quality of housing stock were also aspects which were discussed in another interview with a South Wales Chief Officer:

'...I think we're the third... largest county, I mean some of the merged agencies now are um I think bigger, but I think technically we are a bigger county... there's quite a 
lot of properties out there in the rural areas which are in a very bad state...I'm not just talking about an outdoor toilet, er literally the roof's caving in and there's no water system...' [CO5].

Examples of poor housing stock were also present in a North Wales Chief Officer interview who stated that one of the two counties that this agency covers:

'... I think has got one of the top 3 deprived areas in Wales... but generally you look at it and the thing that horrifies you is when you see some of the houses... that are so bad that they're unfit for human habitation, yet people are living there' [CO 12].

The theme of the presence of a poor housing stock was also highlighted by a Technical Officer:

'And in Wales in particular the housing stock is deteriorating far worse than it is in England...' [TO2].

A practical example was also provided in the focus group where another TO explained that while working on the front line of service delivery:

‘...we still find people with no heating, no inside toilet, often no hot water. The house is in a very deplorable state and some are surviving. It's very, very sad' [TO7].

These interview extracts allude to how deprived areas of Wales with poor housing stock quality is challenging for service delivery, not only in meeting the housing need of the clients, but in a logistical sense; delivering services to individuals who live in relative isolation where an insufficient infrastructure exists to address the needs of the client base. In addition to the theme of rurality, lack of funding to address the poor housing stock which makes the local context in which some agency Caseworkers are operating more complex. This may mean that depending on the agency location, Caseworkers may need to link with a greater number of services to meet their client's needs and the quantitative results about the breadth of organisations (see Table 6.4) for North East Wales, has indicated this. For instance, it could be posited that the North East Wales Caseworkers may have the highest mean score of connections with external organisations as a way of overcoming challenges of poor housing stock, rurality and a restriction of funding for public services.

Another Chief Officer also spoke about the condition of the housing stock in the two counties that is covered by their agency and the solutions available to their clients due to the funding streams available to them.

[Both counties are] 'Highly deprived areas, a lot of old council properties...that have been purchased obviously as time has gone on... A lot of our properties are terraced properties, old terraced properties in need of...general maintenance, general up keep... we are seeing a massive difference in the both areas based on the solutions available, [CO 8$]$. 
The same Chief Officer then went on to discuss the differences between the two counties that her agency covers. This occurrence is not just limited to South Wales; another Care \& Repair Cymru agency which covers two counties of North Wales also spoke about the differences in funding accessibility the Caseworkers can access between counties:

'... we get money from [one county] that we don't get from [another county] ...' [CO 12$]$.

Another South Wales Chief Officer also spoke about the accessibility of funding streams. However, in contrast to the previous instances where deprivation meant that Caseworkers struggled to access funding streams, in this area the opposite appeared to be true. The more deprived areas were able to access more funding streams and grants compared to the pockets of affluence,

'... in the more deprived areas 'we're able to access far more grants... there are pockets, big pocket of deprivation in [more affluent area of county] but it's a bit odd because we can't accept many grants because it's classed as a richer area...' [CO9].

One Chief Officer had a contrasting experience compared to those stated above. This individual who worked in South Wales stated that due to the nature of the relationship between the agency and the local authority meant that Caseworkers could access certain funding streams:

'I know that's not the case in some areas er but because of our relationship with the local authority, they're able to give us access to certain funding streams...' [CO5]

The two latter quotes show how access to funding can vary significantly across agencies. For instance, some agencies will be able to access more funding streams through local authorities, however, others who do not have these types of relationships may be required to develop and maintain links with a variety of external organisations to continue to meet the needs of their clients.

This notion and reality of relative poverty is not limited to this one county and in some instances can be accompanied by other issues including health provision and local authority funding. One South Wales Chief Officer spoke about his region as being:

'...one of the poorest local authority areas in Wales. Really down at the bottom end of the league...on some instances... with income...ill health and er age as well' [CO1].

A lack of funding may mean that Caseworkers must contact several different organisations to find solutions that address the needs of their clients. 
In sum, the quantitative data illustrated agency location is associated with varying breadths of connections between Caseworkers and external agencies, and provided different perspectives underpinning the regional variation in the breadth of connection with external organisations that Caseworkers are making. These may be attributed to the different role requirements of the Caseworker role depending on their location, access to funding or funding priorities, relative deprivation and poor housing stock.

Clients unhappy about changing their home environment: the last significant contextual factor which significantly influenced the breadth of connections that Caseworkers make with external services was the issue of client is unhappy about changing their home environment'. This factor is associated with the breadth of connections Caseworkers make with 6 out of 16 of the different types of external organisations (this includes re-enablement, health, domestic help, digital inclusion fuel poverty and fuel safety organisations). The main findings are highlighted in Table 6.5 below:

Table 6.5- Summary of the key findings: mean scores for association between the issue of 'clients is unhappy about changing their home environment' and breadth of connections with external organisations

\begin{tabular}{|c|c|c|c|c|c|c|c|}
\hline \multicolumn{8}{|c|}{ Type of organisation } \\
\hline & & $\begin{array}{l}\text { Re- } \\
\text { enablem } \\
\text { ent }\end{array}$ & Health & $\begin{array}{l}\text { Domestic } \\
\text { Help }\end{array}$ & $\begin{array}{l}\text { Digital } \\
\text { Inclusion }\end{array}$ & $\begin{array}{l}\text { Fuel } \\
\text { Poverty }\end{array}$ & $\begin{array}{l}\text { Fuel } \\
\text { Safety }\end{array}$ \\
\hline \multicolumn{2}{|c|}{$\begin{array}{r}\text { Total number of } \\
\text { possible } \\
\text { connections with } \\
\text { external } \\
\text { organisation }\end{array}$} & 14 & 27 & 23 & 6 & 20 & 11 \\
\hline \multicolumn{2}{|c|}{$\begin{array}{r}\text { F-value and p- } \\
\text { value }\end{array}$} & $\begin{array}{l}(\mathrm{F}(1, \\
53)= \\
6.62, \\
\mathrm{p}=.013\end{array}$ & $\begin{array}{l}(\mathrm{F}(1, \\
53)=11 . \\
22, \\
\mathrm{p}=.001\end{array}$ & $\begin{array}{l}(\mathrm{F}(1, \\
53)= \\
4.44, \\
\mathrm{p}=.040\end{array}$ & $\begin{array}{l}(\mathrm{F}(1, \\
53)= \\
12.92, \\
\mathrm{p}=.001\end{array}$ & $\begin{array}{l}(\mathrm{F}(1, \\
53)= \\
4.98, \\
\mathrm{p}=.030\end{array}$ & $\begin{array}{l}(\mathrm{F}(1, \\
53)= \\
4.72 \\
\mathrm{p}=.034\end{array}$ \\
\hline $\begin{array}{c}\text { Mean } \\
\text { score } \\
\text { (m) by }\end{array}$ & $\begin{array}{l}\text { Yes } \\
\text { 'experie } \\
\text { nce } \\
\text { issue' }\end{array}$ & $\begin{array}{l}3.97 \\
(\mathrm{SD}= \\
2.17)\end{array}$ & $\begin{array}{l}5.44 \\
(\mathrm{SD}= \\
2.74)\end{array}$ & $\begin{array}{l}2.50 \\
(\mathrm{SD}=2 . \\
72)\end{array}$ & $\begin{array}{l}2.44 \\
(\mathrm{SD}=1 . \\
13)\end{array}$ & $\begin{array}{l}4.39 \\
(\mathrm{SD}=1 . \\
78)\end{array}$ & $\begin{array}{l}2.47 \\
(\mathrm{SD}=1 . \\
06)\end{array}$ \\
\hline $\begin{array}{r}\text { experien } \\
\text { ce of } \\
\text { issue }\end{array}$ & $\begin{array}{l}\mathbf{N o}^{29} \\
\text { 'experie } \\
\text { nce } \\
\text { issue' }\end{array}$ & $\begin{array}{l}2.47 \\
(S D= \\
1.81)\end{array}$ & $\begin{array}{l}2.74 \\
(S D= \\
3.02)\end{array}$ & $\begin{array}{l}1.11 \\
(S D= \\
1.29)\end{array}$ & $\begin{array}{l}1.32 \\
(S D=1.0 \\
6)\end{array}$ & $\begin{array}{l}3.21 \\
(S D=2.0 \\
2)\end{array}$ & $\begin{array}{l}1.84 \\
(S D=1.9 \\
6)\end{array}$ \\
\hline
\end{tabular}

${ }_{29}$ The values presented in bold have the higher mean value of connections while those in italics represent the lowest mean scores of connections.

Post hoc tests could not be conducted because some of the groups have fewer than two cases. 
Overall, Caseworkers who experienced the issue of clients being unhappy about making changes to their home environment made greater breadths of connections with the 6 types of organisations, while those who did not encounter this issue had lower mean scores of connections.

As with agency location, post hoc tests could not be conducted because there were fewer than two cases in some instances. However, the results of the analyses illustrate that service delivery is associated with the local context or issues on the ground. For instance, the types of service links which are influenced by the issue of 'client is unhappy about changing their home environment' are services which can meet a client's needs without a client having to make considerable adjustments to their homes. This is important because it illustrates that the linking actions that Caseworkers undertake are in part shaped by the client's wishes and demands, and not just their needs.

\section{Context of collaboration}

The qualitative data reveals other factors which can influence how the Caseworkers link with external organisations. Specifically, the context of collaboration which has been established across the health, housing and social care sectors in Wales was highlighted.

Qualitative interviews with Chief Officers show that a broader national-level context of co-production (creating and delivering services in partnership with other organisations) in which Caseworkers are operating may also shape both the frequency and breadth of connections they make.

A south Wales Chief Officer notes:

'...co-production and increasing the need for strong partnership is coming in, is at a time where funding streams are being increasingly reduced... restricted...' [CO4].

Some regional agencies however appear to have good working partnerships with other organisations:

'...we've got really good working partnerships with the other organisations we refer into them and they refer into us as well um and we find that works really, really well because it gets the best results for the client...' [CO12a].

In contrast, some Chief Officers spoke about the difficulties in attempting to form working partnerships with certain organisations. 
'...I would say the charitable funding has been much more difficult over the last few years to get charitable funding for clients' [CO9].

This may provide some explanation as to why Caseworkers in certain agencies (see Table 6.4) contact a higher number of charitable organisations:

'Well we are told through Welsh Government and different conversations what funding is you know drying up, it's becoming more harder and reducing er and to look at alternative ways of ... sustaining services' [CO6].

Although funding was discussed in the context of collaboration and delivering services, some Chief Officers also spoke about how it directly affects the Caseworker role:

'... if we take staff on now it's really difficult, you have to do a 1-year contract you know rather than... a permanent contract... and I don't think that is particularly good for morale' [CO9].

In instances where Caseworkers are working on fixed term contracts, it may be difficult to continue developing working partnerships or building up networks if they are no longer working as Caseworkers.

\section{Referrals}

During the qualitative interviews with the clients, the theme of referral arose, and provides additional understanding of the links that Caseworkers make with external agencies, in terms of outcomes for clients.

Clients referred to notions of trust and the reliability of the referrals that Caseworkers made for them. For instance, a client described the referral process in the following terms;

'...you see the Care and Repair system, the way it's set-up, is that they have these contacts of contractors who have a lot of business from Care and Repair. So therefore, they don't charge extortionate prices for people if they have to pay. So that is one of the beautiful things about it...' [Participant $7 \mathrm{a}]$.

The breadth of professional partnerships with external organisations that Caseworkers develop mean that Clients can get in contact with approved contractors who are reliable and charge reasonable prices for the services rendered.

Another client participant commented that the way Care \& Repair Cymru agencies work and communicate with each other and their partners helps to deliver an efficient and effective service.

'They're [Care \& Repair Cymru staff and external services] a well-knit group. One was consulting with the other and things were progressing quickly' [Participant 8]. 
Inter-organisational working benefits clients in a practical way as they experience a more efficient service.

In summary, the breadth of connections made by Caseworkers is also associated with agency location, length of time in role and the contextual issue of clients being unhappy about changing their home environment. The qualitative data has developed our understanding of how these factors affect the breadth of connections that Caseworkers make. Furthermore, there are tangible outcomes and benefits of the external service links that Caseworkers link which has been highlighted in the client interviews. These results will be discussed and interpreted further in chapter seven.

\section{Chapter summary}

This chapter has presented the results of the qualitative and quantitative analysis which explored the external linking activities that Caseworkers undertake to deliver services to their clients, and if these are associated with individual (capital) or contextual factors.

The first part of this chapter examined the types of services that Caseworkers link with. The chapter then presented the results of the statistical analyses which explored associations between the frequency of contact and previous work experience and contextual variables. This was corroborated with qualitative data from the Chief Officer interviews and the Technical Officer focus group where applicable. The results indicated that Caseworkers who had previous experience in public servicehealthcare sector and in managerial roles tended to make less frequent connections (with the exception of previous work experience in managerial roles and frequency of contact with personal support services). Additionally, if Caseworkers encountered changing eligibility criteria for benefits claims as an issue impeding their work also tended to make more frequent contact with certain services.

The final section of this chapter presented the results of the analyses which examined associations between agency location, length of time in role and contextual variables and the breadth of connections that Caseworkers used. The results showed that these the breadth of connections with external services varied by location and the length of time that Caseworkers have been in post. Those who have been in post less than 1 year have the lowest mean score of connections which are significantly less than some of their other peer groups. Finally, the statistical analyses indicated that the clients' perspectives and opinions regarding changing their home environment was 
associated with the breadth of connections made by Caseworkers. CWs who experienced this issue tended to have higher mean scores of connections with external organisations.

In sum the findings from this chapter illustrates that personal attributes (capitals) and contextual factors can shape how Caseworkers link with external organisations and deliver services to their clients. This will be explored in greater detail in the discussion chapter. Chapter Seen will present the findings exploring the affective aspects of the Caseworker role which arose from examining the qualitative data. 


\section{CHAPTER SEVEN}

\section{Presentation of Findings - Part Three: The 'Care’ Aspect of Care \& Repair}

Thus far, the findings chapters have focused on the practical aspects or actions that Caseworkers undertake to achieve the 'repair' objectives of their roles. In contrast, this chapter will explore some of the findings pertaining to the caring aspects of the Caseworker role. What follows is a presentation of the findings of how emotion work is manifested in the Caseworkers' job role from the perspective of staff (Caseworkers, Technical Officers and Chief Officers), and then from clients in addition to some of the perceived outcomes of this emotion work.

\section{Section One: Emotional engagement of Caseworkers from Care \& Repair Cymru's perspective}

Prior to the data gathering phase, a few days were spent shadowing, observing and speaking with Caseworkers to better understand the type of activities required of them as part of their job role. During these initial shadowing activities, it was observed that although interactions were somewhat controlled and professional, the nature of the relationships that Caseworkers develop with their clients appear to be genuine, and the emotional exchange that takes place should be explored in more detail using social theory (emotion work see Chapter Two).

This affective dimension that the Caseworkers undertake was difficult to capture quantitatively, but it is important to acknowledge and examine this aspect of the Caseworker role for two reasons: (1) this was a theme which was identified in the Caseworker questionnaire when the Caseworkers were asked if there was anything that they do which 'goes the extra mile', and if there was anything that could aid our understanding of their role and the work that they undertake. This indicates that the Caseworkers view caring as an important part of their role and this should therefore be acknowledged. (2) it is important to address this aspect of the Caseworker role to generate a holistic view of the role and recognise poignant findings that arose from the qualitative data. (3) emotion work appears to represent a key aspect of the Caseworker role, however, apart from referring to empathy (England \& Farkas, 1986; Rafaeli \& Sutton, 1987) and emotional support (Erickson, 1993, 2005; Vassilev et al., 2013), there appears to be no specific examples of how emotion work is achieved in 
practice e.g. more general statements about actions or processes are used. The qualitative result builds upon and adds to the social theory of emotion work.

The themes discussed were identified in the face-to-face interviews with individual Chief Officers; focus groups with Technical Officers and Caseworkers, as well as the open-ended sections from the Caseworker questionnaire (see Chapter Four). After transcribing the data by hand and reading through the written data, several recurrent themes were identified. These were confirmed using a word frequency search in NVivo, and by cross-checking themes with the supervisory teams.

\section{Building up a relationship with a client}

One of the prominent themes identified in the qualitative data was the recognition of the importance of Caseworkers building a relationship with their client through faceto-face contact and going the extra mile for their clients. The online questionnaire asked Caseworkers to give examples of going the extra mile ${ }^{30}$ and Technical Officers and Chief Officers also alluded to this.

One Caseworker gives a very clear example of how they have exceeded their job description when asked in the questionnaire to describe instances where they have gone 'the extra mile. The extract below shows that to facilitate the best possible outcomes, the Caseworker prioritises building relationships with a client by focusing on emotional engagement, taking an interest in certain aspects of their personal life e.g. family, and using an informal communication style. Effectively they recognise the importance of emotions and actively engage on an affective level to build personalised relationships with their clients.

'I call to check up on client's even after the case is closed, I do this if I know they are particularly alone or going through an emotionally difficult time. I do this because I think as a Caseworker you bond with the clients; we are the only ones they have sometimes.... I try to make the effort with nervous or reluctant clients to get to know them more personally, I feel this helps with the Caseworker relationship, I will always ask about their weekend, or the grandchildren etc. This is what makes me love my job - without it, we would all be robots - I think it is important our client's feel like they're talking to a human, with natural emotional responses - to enable us to get the best outcome whilst promoting independence as much as we can' [NEW9].

\footnotetext{
${ }^{30}$ Q. 22 Like many jobs, there may be several things that Caseworkers do to "go the extra mile" in helping to support their clients, which are not reflected in their job description, but which contribute significantly to helping improve outcomes for clients. Please write down your thoughts about this in as much detail as possible using the box below:
} 
On the face of it, this results in relationships which appear to be like those between family and friends, more akin to an informal relationship. Another aspect of relationship building is ensuring face-to-face engagement with clients, which Chief Officers consider to be fundamental to service delivery;

'I think the work we do, you can't do over the phone with people... if I had to pick one key task for the Caseworkers, it's that, it's that engagement, being able to build that rapport...' [CO 13].

'They're [Caseworkers] an integral part of that and I think breaking the ice with that particular individual er before one of the ugly fellas goes out before one of the Technical Officers goes out and upsets them forever. It broke the ice...' [ $\left.\mathrm{TO}_{2}\right]$.

These extracts illustrate how this contact is valued because it provides opportunities for communication and is the foundation for building these relationships and allowing other services to complete their tasks. The outcomes that Care \& Repair Cymru aim to achieve cannot be done online or over the phone.

A theme of developing trust within the client and Caseworker relationship was also alluded to by Chief Officers and Technical Officers;

'There's a lot of, you know, you are going into someone's home and there's a lot of trust around that you know you've got to build up a relationship with the client as well' $[\mathrm{CO} 10]$.

'...I think it's important to have the social skills to be able to get Mrs Jones to trust you erm, rather than go in because there are a lot of questions that we need to ask... They are very personal questions...' [CO8].

'The Caseworkers' job is to find out exactly what that client's needs, wants first but then needs, and to do it in a way that they build up a bond with that client and not a friendship but a trust' [CO9].

'... they need kind of to build that trust in us that you know we've helped, and we can do it...' [CO12a].

'Tou build up that relationship and that trust erm and it does make the role of Care \& Repair quite unique and difficult to get out off I feel at times' [TO 1].

These extracts give the impression that developing a sense of trust is fundamental to these relationships.

\section{Empathy}

Chief Officers also alluded to another emotional dimension of the Caseworkers' interaction with clients, the ability empathise with their situation. Having and using empathy is a recognised component of emotion work theory (England \& Farkas, 1986) and successful workplace interaction (Rafaeli \& Sutton, 1987). Chief Officers 
were asked during interview to comment on how they would present the Caseworker role to a new recruit (see Appendix item 4);

'...somebody who comes out who is understanding, who I think the client can relate to in terms of this person's actually hearing what I'm saying and, I'm kind of saying I'm struggling with these things and this person is listening to me and not saying, well we can give you, this, this and this, actually they're hearing what I want and what I need as well' [CO12a].

Characteristic of this type of empathy would be a Caseworker's ability to listen to people and respond to their needs and worries in an appropriate manner. One Chief Officer commented;

'...the fact that she's worrying about it, is enough and it's the empathy and the understanding that that Caseworker brings to that situation that can turn it round and to stop it turning into something you know far bigger' [CO6].

From a Caseworkers' perspective, being empathetic involves making client's feel valued by building a relationship of trust, and by taking time to listen and understand the issues which the client's themselves consider important;

'As a Caseworker you never know when you first visit a property what you are going to find behind that front door. Being able to assess a client by giving them time to talk and showing interest in their lives goes a long way to gaining their trust and getting to know what really matters to them. By following through with your promises, addressing the problems they want you to, not what you think they need and keeping them informed can make all the difference in making that client feel valued'. [NEW 7].

One Technical Officer alluded to the pressure and stress that can arise from empathising with client's personal situations and attempting to put solutions in place;

'It is truly stressful when you see these people... if you've got any empathy with them, it's incredibly stressful and on top of that you've got the stress of have you got the CDM bit right you know, because... you're focussed on getting something done for that individual, doesn't matter how small...' [ECR\&C]

While another Technical Officer and Chief Officer alluded to how failing to deliver services or help clients can cause feelings of stress and strain;

'...it is difficult and sometimes I can imagine most time [Technical] officers go home quite stressed and the pressure on them, thinking I've got to try and deliver this... [TO6].

'We do what we can, but we can't do everything, and that's the difficulty, and this is what I say to Caseworkers... sometimes they come back and it's a bit demoralising to not be able to do what they want to do and as long as they do their best, what more do you want them to do? [CO15].

Part of relationship building is therefore a Caseworker's capacity to harness empathy by listening, understanding their client's needs, making clients feel valued and 
stemming any client anxiety. However, an outcome of using empathy for the Caseworkers and Technical Officers are feelings of stress.

\section{Going the extra mile...Caseworker perspectives}

Caseworkers provided practical examples of going the extra mile and these included both affective and more instrumental tasks. In addition to showing empathy by listening to people and recognising the importance of communication, one Caseworker also helped her clients with very practical tasks;

'...just listening to people, spending the time to have a bit of a chat, particularly with those that don't leave the house often for mobility or mental health reasons. Sharing a joke. Being non-judgemental... Other little things as well, such as putting their rubbish out while I am there as they don't have the strength to lift bag out of bin and carry outside. Lots of other little things that can't remember off top of my head' [NEW2].

Others allude to the importance of continuity of care along with listening and reassuring clients; all of these entailed working beyond normal contracted hours:

'A Caseworker will contact a client and check all is ok, and won't close the case till everything is ok, e.g. call on the way home, ring up as often is needed. Offer reassurance and a listening ear at all times' [NEW4].

'I generally go that extra mile for clients and do as much as I can in order to help them which usually means eating into my own time, working later in the office or at home'. [Carm1].

One Caseworker refers directly to the need for time to complete her work and how this can spill into weekends;

'... some time over visits to help meet all clients' needs... less time for lunch sometimes. Taking phone calls over weekends on occasions. Being overworked and stressed to ensure clients' needs are being met. (It would be much easier not to ask questions to avoid the extra work but would never do this)' [Gwy\&Ang3].

Another spoke about engagement which supplements a lack of family support for social care needs (additional responsibility);

'Over the years I have done many things that are not on my job description, for example, I have visited a client who was not well and had no family members and ended up doing her food shopping before arranging for a Care Agency to come in, booked hospital transport, fitted a new zip in a pair of trousers, sang hymns and played the piano!!!' [Con\&Den2].

Technical Officers also spoke about supplementing family support by project managing building works and attempting to take away the stress from their clients;

'We hope by our involvement that they don't feel it's too onerous to go ahead ... hopefully by the time they've got to know you and trust you they feel that with you organising it and overseeing it and taking care of all the things that families did, 
that they literally you know just have to sit there and wait for the outcome... we try and improve their lives without having the stress...' [TO 1].

Another Caseworker spoke about how caring and emotional engagement has become a core component of their work in supporting clients;

'The issue may not be linked to housing repairs or adaptations either; we are more and more assisting clients with their wellbeing and feelings. I feel the Caseworkers at Care and Repair [agency] go above and beyond the call of duty on a daily basis' [NEW9].

From these examples it is clear that Caseworkers do become emotionally invested in the relationships they create and build with their clients;

'Tou do get attached to people especially if you've been working with them for a while, it's really sad then, you feel a personal loss when they die; especially if you've been helping them for years' [Caseworker in focus group].

This notion is supported by the reference to experiences of stress by one Caseworker.

A Technical Officer also talked about the strain that Caseworkers can encounter through their work;

'It can be very, very stressful for the Technical Officers. Caseworkers have a similar situation because Caseworkers and Technical Officers, we're the front line. We're the people who go out there and meet the clients, meet the sad clients, the emotional situations, the terrible conditions, we're front line and we have to deal with it.' [TO7].

However, despite the stress that can arise from this sort of work, one Technical Officer stated,

'...This is the most stressful job I've ever had but it's also the most rewarding one' [TO8].

This provides an insight as to why the individuals working in Care \& Repair remain in their job roles despite all the challenges that they are face, as was evidenced in the quantitative analysis of the Caseworker study population, in which just over half of all Caseworkers (52.7\%) had been in post for $5+$ years.

However, taking additional responsibility also extended to individuals who could not become clients because they were not part of Care \& Repair's remit. This could entail helping clients despite them not fitting into their criteria or acting as brokers and putting the clients in contact with other services;

'We very rarely turn a client away, even if they are outside of our criteria, if they are in need, we find a way of helping, this may be as simple as referring to an organisation who can assist or actually helping ourselves to ensure the problem is solved' [NEW9].

One Chief Officer alluded to innate attributes in addition to empathy and other skills as being fundamental to the Caseworker role; 
'...you've got to have the right person as a Caseworker, you've got to be very understanding, very empathetic because it's no good treating it as any other job because it's not; you've got to be a special type of person and you want to help somebody... it's about the whole package' [CO9].

In summary, the qualitative data derived from the interviews, questionnaire and focus groups with Care \& Repair staff provides tangible examples of how emotion work is enacted by the Caseworkers, and the recognition of its importance for service delivery. From a staff perspective, emotion work involves building a relationship of trust with clients. This is perceived to be an important element of the Caseworkers' role which can only be achieved through face-to-face interaction and emotionally engaging with clients. Emotional engagement is also viewed as a fundamental component of this process. The nature of these relationships appears to be like those between family and friends where Caseworkers will take on additional responsibility for their clients, encompassing both instrumental and affective tasks. Staff view going the extra mile for clients as taking on additional responsibility which at times goes beyond the Caseworkers' job remit. However, the staff acknowledge that a consequence of the nature of their work and approaching the clients in this manner is that they encounter stress and feel personal loss if their clients pass away. The findings from this section allude to the way of working (emotion work), and the unique, almost overly personal relationships Caseworkers form with their clients.

\section{Section Two: Client experiences of the services}

The previous section looked at different dimensions of emotion work from the perspective of staff. This section now examines the perspectives of clients who have used the service to see if these same elements of emotion work are present, in addition to exploring the outcomes of the Caseworkers' emotion work.

\section{Trustworthy and professional service}

\section{Trust and reliability}

Two interrelated themes that arose from the client interviews were the notions of trust and reliability. Participant 3, a female living on her own, alluded to the trust that Care \& Repair as an organisation have built into their reputation;

'But [friend] said to me, because he knows [Caseworker], and he said to me, "Look now, I'll give you [Caseworker's] number. Give her a ring. Now, she's a good one." They've got an excellent name you know?' [Participant 3].

Following this, participant 4, an older lady living on her own, alluded to how this Care \& Repair Cymru agencies' reputation extends to their recommendations and 
links with trusted services and contractors. This suggests that Caseworkers use their position to act as brokers between clients and other services;

'And if they can't do it, they suggest somebody trustworthy to do it. That is the secret, because otherwise you're in the Yellow Pages and you don't really know what you're looking for' [Participant 4].

This issue was also referenced by Participant 9, a woman living on her own with back and spine issues following a fall, who was referred to Care \& Repair by social services. She has since used Care \& Repair to access financial assistance which could be used to address home maintenance issues. However, she also commented on Care \& Repair being a trusted and reliable organisation to contact if any issues arose;

'I suppose at least you know that the tradesmen coming in are trusted and... If you did have any problems you can, you have erm someone to ring and contact' [Participant 9].

Finally, Participant 6, a single visually impaired lady living on her own, spoke about the reassuring presence the Caseworkers have given her considering her personal circumstances of moving to a new area and not having a developed network of personal support;

' $Y$ ou can trust them and rely on them, which makes a lot, a lot of difference to me. I've got somebody that I can trust and rely on' [Participant 6].

These extracts highlight the interconnectedness of the outcomes of relationships that Caseworkers form with clients. Through creating relationships of trust, clients feel reassured that they can access services which are trustworthy and that the presence and accessibility of Caseworkers provides support.

In sum, while trust does not appear in the emotion work literature, it represents one of the processes needed to develop positive relationships with clients, as well as an outcome of the emotion work undertaken by Caseworkers.

\section{Practical knowledge and expertise}

Client participants also referenced the functional dimension of the Caseworker role, in particular the knowledge and expertise used by Caseworkers to guide clients through complicated and arduous processes. Participant 1a, a lady who was part of a married couple stated that;

'Things we, we didn't have a clue about; she guided us through everything you know? [Participant 1a].

Additionally, Participant 5, a divorced man living on his own with quite severe mobility problems, which were mainly caused by back surgery which was undertaken a few weeks prior to the interview. Due to this client's reduced mobility a buzzer, 
intercom and electric door entry system were installed into his home. When asked about it he responded that his local Care \& Repair Cymru agency;

'...recommended that. I must say, it was talked about and the next moment it was all installed, which was very good, and the system works very well... They've been very, very strong on the advice to be given and to the limitations that I would have...' [Participant 5].

Participant 9 also alluded to the specific types of knowledge that Caseworkers have which can be immensely useful for clients;

'[Caseworkers $]$... really inform you of what's available' [Participant 9].

The specialised knowledge that the Caseworkers have can be used to give impartial advice or to achieve practical outcomes or advise on future processes. This is best summarised by Participant 7a, a married man living with his wife, both of whom had health and mobility issues;

'The point is, without [Caseworker], or not only [Caseworker] but somebody who was knowledgeable as [Caseworker], knew exactly what people were entitled to...We wouldn't have known where to turn, seriously...' [Participant 7a].

The theme of the Caseworkers' expertise was also addressed in the Chief Officer interviews;

'... almost like being a jack of all trades, I wouldn't say master of none because you are very much a knowledgeable source of expertise' [CO4].

This knowledge and expertise can be used by Caseworkers to achieve practical and beneficial outcomes. This is highlighted in another theme which was discussed by Caseworkers, which was financial support application processes.

\section{Follow-up service: Caseworkers 'being there' for clients}

The type of relationships that clients and Caseworkers form with each other is discussed in a different manner by the clients. In addition to focusing on the outcomes of these relationships, clients also alluded to the longevity of their relationship with Caseworkers and how this led to certain outcomes discussed below.

\section{Emotional engagement of the Caseworkers: client's perspectives}

While the Caseworkers place a priority on taking an interest in their clients and engaging with them emotionally to develop relationships; the outcomes of these actions appear to be appreciated by clients. Participant 8 , a married gentleman whose wife had several health and mobility issues, said;

'Circumstances have led us that way and [Caseworker] has taken an interest by coming along to see how [wife] is getting on and the other ladies are coming here' [Participant 8]. 
Participant 8 went on to say how the services offered by his local Care \& Repair Cymru agency exceeded his expectations and that due to his experiences he will be returning to them if he is in need.

Similarly, Participant 6 discussed the caring and supportive relationship that she developed with her Caseworker. This was reflected through the Caseworker taking an active interest in the wellbeing of this participant and being accessible whenever needed;

'...she's back and forth asking me how I am, how I'm getting on, do I need help? She's there... she has been there if I wanted to talk to somebody. She said, "We're here with you now, all the time. This is our job," and they go beyond their job actually...there is always that-I don't know- the connection there with them. It's not like somebody coming in and just saying, "Yes, we'll do this, and we'll do that." They do care, and they will do it' [Participant 6].

These extracts illustrate the complexity of the relationships that Caseworkers and clients develop. In many ways, in line with the assertion made earlier, the relationships appear to be similar in nature to those found within families. While staff view emotional engagement as a key aspect of the Caseworker role, it appears that the reality of the service from client's points of view match this. The affective tasks undertaken by the Caseworkers are viewed by clients as going the ex tra mile for their clients. The following sections now outline the perceived outcomes of the relationships that clients and Caseworkers develop.

\section{Alleviating worry and stress}

A prevalent theme was the alleviation of stress, where the relationships developed with Caseworkers provided some form relief from worry. For example, Participant 2, a married woman with mobility issues living with her husband who was suffering with dementia, described the outcomes of contacting her local Care \& Repair agency. Aside from the practical outcomes including having a greater income, undertaking repairs and maintenance works on her home e.g. having a new boiler fitted, she also spoke of how her emotional wellbeing had improved;

'I just think they're amazing people... Like I said, they were amazing. They took everything completely. They took all the weight off my shoulders and made life much easier' [Participant 2].

The quote above illustrate that clients recognise that they have had the stress "taken off them” by receiving help from Care \& Repair. Quite often, participants would relay that because the sense of stress and relief had been taken away from them; that their 
lives felt as though they were easier and less pressurised. This is illustrated in the quote below:

'... it has made my life a lot easier... they've taken the pressure off me; a lot of pressure and they've done things for me which is very good' [Participant 6].

The alleviation of worry and stress does link into a sense of reassurance. The reassurance that there is an organisation or individuals there for the clients to rely on or go to for specific issues does appear to alleviate stress. This is illustrated in the quote below:

'Puts our mind at rest to think that there's somebody there that's going to help...' [Participant 7b].

The idea of alleviating some of the worries and stresses faced by older people throughout Wales was also commented upon in the Technical Officer focus groups. In speaking about delivering front line services one Technical Officer asserted that:

'...to take some of the stress out of it for them [older people]. We hope by our involvement that they don't feel it's too onerous to go ahead...' [TO1].

This could also be understood as a form of emotion work in which the Caseworkers are sharing the burden of their clients' issues to alleviate stress and worry while finding solutions. One client even spoke about what would have happened without the help they received from their local Care \& Repair agency.

'Oh, she did everything for us, fair play. Everything that I needed to do, I wouldn't have had anything, and I would never have claimed either' [Participant 2].

Examining this excerpt, it could be postulated that this client may have not applied to any of the help that she was entitled to because of the stress associated with the processes. However, her assigned Caseworker intervened and helped this client to maximise her income.

The quotes examined in this section illustrate that an integral part of the front-line services of Care \& Repair agencies is to alleviate some of the stress that some older people face in their daily lives. Additionally, many of the clients interviewed stated that this was a highly valued aspect of the service which provided them with a sense of reassurance and ease when confronted with what can be arduous processes.

\section{Confidence and reassurance in service delivery or contractors}

Another of the recurrent themes that arose from the client interviews were sentiments of confidence and reassurance stemming from their relationships with Caseworkers. One female client living on her own claimed that asking her local Care \& Repair Cymru agency for recommendations for approved contractors has given her 
a sense of reassurance that a trusted organisation has vetted several local contractors and services;

'It's given me confidence, yes. It's given me confidence, absolutely' [Participant 3].

The same client along with others referred to the confidence they felt from being able to readily access their Caseworker.

'It is, it's like a rock for you, you know, to help you... They are, they're available, you know? They're really good, I've got to say' [Participant 3].

A similar theme arose from an interview with another client living on her own who contacted her local Care \& Repair Cymru agency for minor building adaptation work.

'They're like a bolthole. They're always there' [Participant 4].

Furthermore, one client who was a disabled gentleman living on his own commented that having a local organisation who is working to help older people remain in their own homes is reassuring;

'It's been so reassuring to think that there's a body out there which is operating for you... Basically, it's my helping hand, they're the guardian angel behind it all, sort of thing' [Participant 5].

The statements listed above refer to the Care \& Repair service, but some clients referenced that the Caseworkers gave them a sense of reassurance. For instance, Participant 6, a disabled female living on her own had recently moved into an area where she had few friend or kinship connections. This client had to cope with recent vision loss, bereavement and moving into a house which was not yet suitable to her needs. When speaking about her situation she was thankful that she can rely on her relationships with her Caseworkers;

'I've got somebody there, because as [Caseworker] said to me "If you need anything, if you want to talk about anything, ring me." So, I know if I've got a problem, that's all I've got to do is ring [Caseworker] and she's here... That means a lot...It makes a big difference because you know you've got somebody at the end of the phone... So at least I've got somebody out there that knows everything and can help me, and they've taken it all off me, the stress, everything'. [Participant 6, a visually impaired, lady living alone in Bridgend].

One participant went even further to state that having access to Care \& Repair services can be a lifeline for some older people, especially because there is a form of follow-up. Rather than simply, undertaking and completing one type of intervention, there's a follow-up and consistent service delivery. This provides clients with a sense of reassurance that there is somebody there for them to help if needed.

One participant acknowledged that the continuity of service and care provided by the Caseworkers is reassuring for clients: 
'They're there for us and that's it...And that's the beautiful thing about being introduced to Care $\mathcal{E}^{2}$ Repair, that it's a lifeline if you need it. It's not just a one off and forget it. They're there for you' [Participants 7a].

In sum, from the client interviews, it appears that the continuity of service provision provides a sense of reassurance for the clients. Conversely, from a service delivery point of view, continuity of service appears to be something which the Care \& Repair agencies strive to achieve.

\section{Emotion work and the Caseworker role}

The qualitative data alluded to emotion work being part of the Caseworker role, while the staff of Care \& Repair Cymru tended to focus on the processes involved with building a relationship with the clients and addressing their needs, and clients focused on the outcomes of these processes.

For the staff of Care \& Repair Cymru, developing relationships is fundamental to service delivery. This is achieved through emotion work or several processes undertaken to develop positive relationships that enhance the wellbeing of those involved. In the context of the Caseworker role, emotion work entails taking an additional responsibility for clients by undertaking instrumental and affective tasks. The Caseworkers' ability to empathise with their clients is also key to relationship development and earning the trust of their clients.

The client data explores the relationship with Caseworkers from a different perspective by focusing on the outcomes of the Caseworkers' actions. Specifically, the emotional engagement of the Caseworkers was appreciated by clients in that Caseworkers genuinely cared for them, which provided reassurance that there were trustworthy and professional individuals willing to help them. This also gives clients confidence to contact contractors and third sector organisations that have been recommended to them. Finally, through their connection with Caseworkers the worry and stress of facets of everyday life are alleviated or taken on by the Caseworkers.

Two cross-cutting themes were identified in the qualitative data on trust and alleviating stress and providing reassurance. Trust was viewed by the staff of Care \& Repair Cymru as fundamental to establishing relationships with clients. To achieve this, Caseworkers take on additional responsibility regarding their clients which means engaging in instrumental and affective tasks which are not dissimilar to 
kinship relations. However, trust is also an outcome of the Caseworkers' caring interactions with their clients. The trust gives Care \& Repair agencies and services a reliable reputation which extends to the recommendations to other services that care \& Repair make. This trust facilitates the Caseworkers' role as link agents by linking clients with vetted services that clients can trust. Therefore, while trust is not mentioned in the emotion work literature, in the context of this thesis, developing trust and using it in the formation of relationships is part of the emotion work that Caseworkers undertake.

The second cross-cutting theme was that of reassurance and alleviating worry and stress. Care \& Repair Cymru staff view alleviating worry and stress and providing reassurance as a function of the Caseworkers' job role. This was also the reality from the perspective of the clients. Many of them described the alleviation of worry and stress that had resulted from their relationship with Caseworkers. Therefore, the processes or actions of Caseworkers reassuring their clients and alleviating worry could also be understood as a form of emotion work in which the Caseworkers are sharing the burden of their clients' issues to alleviate stress and worry while finding solutions. This will be discussed in greater detail in Chapter Eight: Discussion and Conclusions.

\section{Chapter Summary}

This chapter examined how emotion work theory applies to the Caseworker role. It did this by examining the realities of the Caseworkers' work from the perspective of staff members and contrasting this with the experiences of clients. This was to determine if the realities of the Caseworker role were observed by clients and to explore the outcomes of the Caseworker intervention. An examination of the qualitative data provides a more in depth understanding of how Caseworkers link with their clients and the skills they employ to develop and maintain these relationships. Several interrelated themes have been alluded to in this chapter which exemplify how emotion work is undertaken and the outcomes of these processes.

The next chapter will discuss the findings from Chapter Five, Six and Seven together and ground them in the literature that has been reviewed in Chapter Two in this thesis and the social, policy and cultural context (outlined in Chapter One) in which this thesis is based. 


\section{CHAPTER EIGHT}

\section{Discussion and Conclusion}

Through its aims and research questions, the thesis set out to examine how Caseworkers are networking with each other, for example as sub-groups, and whether there are any clear patterns and explanations for this. Moving beyond the Caseworker network, it also set out to establish the nature of the links they build with other organisations across Wales and explanations for these. Qualitative interviews were designed to help extend understanding of the relationships formed with clients and the experiences of the Caseworker service from the perspective of clients. Combined, these findings provide an empirical basis for revisiting the notion of Caseworkers as link agents.

The discussion will elaborate key findings which address these aims and questions and ground them in the thesis' theoretical framework of network theory, and human and social capital and emotion work theories. This chapter will conclude by considering the potential for future research in this area.

\section{How Caseworkers are connecting with each other}

Findings have shown that Caseworkers do indeed build different forms of connectivity through their network; they are connecting with each other directly, sometimes as individuals (in dyads - indegree and/or outdegree scores), sometimes as members of sub-groups of 3 or more members (cliques).

On the whole Caseworkers are not making a lot of direct connections with each other as they have relatively low outdegree and indegree scores (3.55 and 2.68 respectively). There are, however, some exceptions to this. As individuals, some can act as what could be called hubs (that is they have higher than average indegree or outdegree scores) because they represent a centre of activity in the network; they make lots of connections with other Caseworkers or their Caseworker colleagues are choosing to link with them. Another type of $h u b$ are individuals who have exceptionally high connections overall within the Caseworker network (combined indegree and outdegree scores). These findings are corroborated by Woodcraft (2011) research on mapping and improving public service delivery. 
There are also Caseworkers who appear to be bridges because they take on brokerage roles within the network by facilitating links between Caseworkers other than themselves (betweenness centrality scores which measure the potential for a Caseworker to act as a broker between two colleagues who are otherwise unconnected). This role in the network is similar to the 'gatekeeper' role described in the work of Stephenson (2005) and Woodcraft (2011) and the 'co-ordinator' role described by Nicaise et al. (2012).

Like high centrality measures (indegree and outdegree), Caseworkers with higher than average betweenness centrality are structurally and functionally important to the structure of the Caseworker network. This is because they occupy strategic positions in the network because they lie on the greatest number of shortest paths between other Caseworkers and therefore have the potential to connect many pairs of otherwise unconnected Caseworker colleagues. Hence, removing them from the network could cause the network to become disconnected and paths between unconnected Caseworkers would become longer.

Effectively, CWs with higher than average betweenness centrality scores have the potential to be key players in the network. This has certainly been suggested in other network studies which have focused on bridges between different networks of services or organisations including Meltzer et al. (2010); Nicaise et al. (2012); and Valente, Corongesa, Stevens, and Cousineau (2008).

Finally, Caseworkers are networking by building sub-groups (cliques). These subgroups are formed from a minimum of 3 Caseworkers who link together and who do not necessarily work in the same agency; the largest sub-group within the Caseworker network contains 6 individuals.

\section{Explaining these networking connections}

As hubs are situated near the centre of a network or in clusters of activity, this suggests that these individuals play an important role in the Caseworker network. This interpretation is supported by network theorists. Borgatti et al. (2013) conceptualise centrality measures (indegree and outdegree) as representing an individual's structural importance in a network. In other words, if $h u b$ individuals with a high number of connections were to be removed, it would leave parts of the network disconnected. In terms of their functional role, Hanneman and Riddle (2005) consider those with high centrality scores as important or powerful in networks 
because they have the ability to spread influence across a network, or have specific attributes which draw others to them. Meltzer et al. (2010) exploration of the usefulness of individual social network measures in selecting individuals to join quality improvement teams in an academic healthcare context in Chicago led them to conclude that an individual's position in a network affects group dynamics and communication depending on the amount or quality of information that they are privy to.

A few Caseworkers had much higher than average incoming links (higher indegree scores for Con\&Den 1 and NEW8). Hanneman and Riddle (2005) suggest that hub individuals with high indegree scores can be considered to have an amount of prestige. Through a capital lens, this prestige represents the resources individuals build including knowledge, skills or social contacts (human and social capital attributes). According to Bourdieu's version of social capital theory, this prestige represents the volume of capital that individuals possess which along with the network size is associated with the volume of social capital possessed by individuals (Bourdieu, 1973). Additionally, in Coleman's iteration of the theory, prestige signifies the sources of information that other Caseworkers can access and make use of to facilitate social action (Coleman, 1988, 1990).

These interpretations give further meaning to some of the empirical findings of this thesis which have shown that higher indegree scores were associated with Caseworkers who had previous work experience in the business sector. In short, the forms of knowledge and skills (human capital), or professional contacts (social capital) that Caseworkers have developed because of their experience of working in the business sector are proving to be assets that other Caseworkers wish to access and draw on for their own work. The prestige referred to by Hanneman and Riddle (2005) is synonymous to this type of knowledge and work experience. This could be highly valued in the current policy context of collaboration, economies of scale and cost effectiveness.

For these hub individuals with a high outdegree scores, Hanneman and Riddle (2005) also suggest it could mean they are gregarious or have the potential to be highly influential because they can reach out to many people within a network.

Social capital theorists including Hawe and Shiell (2000), Lin (2001), Portes (1998), and Woolcock and Narayan (2000), share the interpretation that that social capital 
takes an investment of time and energy to develop. Therefore, it is reasonable to assume that individuals would have a greater number of connections after they have occupied the role for some time, compared to individuals who are new to the post. The findings from this thesis add a new insight to these assumptions because outdegree scores are associated with different lengths of time a Caseworker has been in post. Results show that Caseworkers who are new to the post (6 - 12 months) have higher than average outdegree score than those who have been in post longer. This suggests that new starter Caseworkers invest a lot of time initially building 'outward facing links' to other Caseworkers. This finding certainly fits into the version of social capital theory forwarded by Lin (1999) in which social capital is understood as investment in relationships with expected returns. Indeed, the finding that newly appointed Caseworkers make a concerted effort to develop relationships may reflect the aim of generating certain outcomes or expected returns for these Caseworkers e.g. access to specialised knowledge.

These results may also reflect a changing social context and the need to be more efficient in using the resources available to deliver services to clients. New Caseworkers may effectively be seeking social and human capital resources from colleagues who have been a part of the workforce for a longer period and will have had opportunities to build their knowledge and expertise through workplace training and experience. New starter Caseworkers could effectively be reaching out more than average to their more experienced colleagues to access certain forms of knowledge, skills or professional contact which will be enable them to fulfil their role as Caseworkers.

The findings discussed so far confirm the association between at least 2 dimensions of certain forms of capital (working in the business sector and the length of time a Caseworker has been in post) and patterns of networking. These dimensions represent opportunities for Caseworkers to develop their professional contacts (social capital) in addition to their knowledge and skills (human capital). This interpretation suggests that social and human capitals are not mutually exclusive. However, previous network studies by Ennis and West (2010); Inkpen and Tsang (2005); Lin (1999); Mobius et al. (2004); and Tsai and Ghoshal (1998) mainly focused on social capital and social networks. This thesis has indicated that a more nuanced understanding of human and social capital should be incorporated into social network analysis studies. For instance while networking and social capital go hand in hand (Ennis \& West, 2010), and social capital refers to the capital of relationships and the 
resources embedded within them, these resources are the knowledge, skills or contacts that certain individuals build and others want to access. This should therefore be considered in studies of social networks

\section{Caseworkers' patterns of interaction}

Based on the work of Stephenson (2005); Woodcraft (2011) who identified three different types of networking roles in social networks; this thesis also aimed to determine if the patterns of interactions between Caseworkers could be typified and two distinct groups of connectivity were identified. However, in contrast to the mean centrality scores which indicated that Caseworkers do not make a great number of connections the results from the LPA indicates that the Caseworker study population is characterised by the high connector group containing more individuals, with a relatively smaller group of low connectors.

Most Caseworkers are not making a great number of connections which is exemplified by the low connector group containing more individuals, with a relatively smaller group of high connectors.

Further statistical tests indicated that the only statistically significant factor that was associated with Caseworkers belonging to a high connector or low connector group was whether they had previous work experience in the customer service sector. Caseworkers that have this type of work experience are more likely to be members of the low connectors group. It was surprising to find that no other social or human capital factor could explain group membership. This could be because this empirical measure is too simplistic and inadequate for capturing the reality of how the Caseworkers are connecting with one another.

For instance, in the low connectors group, there are individuals who make connections albeit not very many, but there are also individuals who are not making any connections at all. These nuances are lost. Equally, there is one anomaly in the low connectors group; Con\&Den 1 had 11 overall connections. When it is considered that the mean number of connections is 2.53, the logic of classing this individual as having low connectivity does not make sense.

Furthermore, this Caseworker has an indegree score of 10, which is the maximum indegree score of all the Caseworkers, which suggests that this individual has a degree of prestige about them. In other words, they could be a key individual in the 
Caseworker network because they possess social or human capital which others wish to access. Inclusion in the low connectors group overlooks these possibilities.

Therefore, the nuances of whether Caseworkers have only an indegree score or outdegree score or a combination of both is not reflected in any detail in these two groups.

\section{Caseworkers linking across agencies}

Findings also indicate that networking patterns are not constrained by agency location through the identification of sub-groups (cliques) or agency size by examining direct connections between Caseworkers.

This is shown through the final type of connectivity that moves beyond dyadic links and shows Caseworkers are linking together as sub-groups within the larger network. Many Caseworkers are networking as small sub-groups of 3 or 4 individuals but there are some larger sub-groups containing 5 or 6 individuals. This type of networking is not geographically constrained and illustrates collaborative working between agencies. Of the 56 cliques that were identified in the network, only 12 were same agency cliques. This suggests that although Caseworkers link with colleagues in their own agencies, agency location is not the only factor which could explain their networking behaviour.

As well as geographic location, agency size does not constrain patterns of networking. During a conference presentation, the question was raised about whether agency size could impact the Caseworkers' networking e.g. working in a larger agency means that Caseworkers can access more connections. However, the results examining the direct links made between Caseworkers do not support this assertion. Indeed, the social network analysis indicated that RCT5 had the highest overall score of connections and worked in an agency that had 4 Caseworkers in total. Similarly, Caer3 and NEW3 had the third highest total number of connections in the Caseworker network. But the Caerphilly agency was 3 times smaller than North East Wales in terms of the number of Caseworkers employed. Considering that these two agencies are quite different in size, the Caseworkers nonetheless had the same number of connections.

In sum, this thesis has made several poignant findings regarding how Caseworkers network and collaborate with each other for the purposes of work. This is not 
determined by geographic location and indicates that the Caseworkers' personal attributes influence the shape of the $\mathrm{CW}$ to $\mathrm{CW}$ network.

\section{Working collaboratively with organisations beyond Care \& Repair Cymru}

Moving beyond the Caseworker network, the thesis also assessed the links that Caseworkers said they made with organisations external to Care \& Repair Cymru this involved using other statistical tests of association. These links were studied in terms of the frequency of contacts and breadth of organisations contacted. The purpose was to establish the nature of the links they build with other organisations across Wales and to identify explanations for these.

The findings show that working collaboratively across different sectors is a vital component of the Caseworker role. Overall Caseworkers identified 383 different organisations and 16 reasons for approaching or referring to external organisations including fundraising for clients, increasing the income of clients and addressing health and safety issues around their homes (see Table 6.2). One of the most frequent contacts made was with home maintenance services which one would expect, but Caseworkers also form professional connections with a range of other types of organisations; some of which have to do directly with housing (e.g. slips, trips and falls services), some of which do not (e.g. financial assistance, fundraising). This shows the diversity of their work which involves not only linking with lots of organisations but linking across several sectors. Caseworkers are therefore a strong example of partnership working in action and this extends across Wales.

In seeking to find explanations, what is striking is that the manner in which Caseworkers connecting with each other is not linked with how they are linking with external organisations. Rather the frequency and breadth of connections with external services is associated with different individual and contextual factors.

\section{Factors that influence the number of external organisations Caseworkers connect with}

The analyses indicated that there were three factors which were associated with different breadth of connections with external services.

More 'seasoned' Caseworkers have more external organisation connections The length of time a Caseworker has been in post is associated with the breadth of connections that they make to 6 different types of organisations. Caseworkers who 
had been in post between 1-3 years had the greatest mean breadth of connections with 4 of these organisation types, new starter Caseworkers had the lowest mean breadth of connections with external organisations overall.

This finding finds explanation in social capital theory which recognises that social capital requires an investment of time and energy to build up and maintain (Grossman, 2013; Hawe \& Shiell, 2000; Portes, 1998; Siisiainen, 2003; Woolcock \& Narayan, 2000). It follows that newer Caseworkers would have the lowest mean breadth of connections with some external organisations because they have not been in post long enough to develop a great number of links with them.

Although there was no statistical association between how Caseworkers are networking and breadth of links they make with external organisations; this could explain why new Caseworkers reach out to their colleagues within the Caseworker network. As a group they have the lowest breadth of connections with external services, but the highest scores of outdegree. This high level of networking may represent their attempts to build their external links by reaching out to their more experienced colleagues to access the contacts that they have developed.

The findings also show that in terms of time in role, it does not take the Caseworkers a great length of time to build up the number of collaborative partnerships they have. Indeed, after being in post for a year they have some of the highest breadth of connections with external services. This suggests that the way these relationships are built or the sharing of social capital throughout the Caseworker network is highly effective.

\section{Regional variation in the breadth of connections made by Caseworkers}

Agency location was associated with the breadth of connections Caseworkers made with external services. For example, of the 15 agencies included in the analyses, the agency covering North East Wales had the highest or second highest mean score of connections while Newport has the lowest mean score of connections for all but one type of connection. What explanations or factors might be causing this?

First, the Chief Officer themselves reaffirm from a qualitative perspective that geographic location impacts external organisation links. There are several explanations which could underpin this finding but overall this indicates that the 
local context in which Caseworkers are operating does impact how they connect with external organisations.

One explanation is that different forms of social and human capital are concentrated in different agencies, suggesting an uneven distribution these capitals or hotspots of expertise across the organisation.

Another explanation which arose from the Chief Officer interviews was that the Caseworkers' job description varies between agencies. This was reflected in responses to the Caseworker questionnaire, which showed in their role, some bring more 'specialist' and focusing on achieving specific or specialised outcomes for their clients. For instance, in the North East Wales agency there was a 'hoarding Caseworker' whose role was to support older individuals who had hoarding tendencies. This may result in Caseworkers linking with external organisations in different manners.

The strategic mission of local Care \& Repair Cymru agencies can also influence how the Caseworkers are linking with external organisations. For instance, the analyses indicated that the Swansea agency had the highest mean breadth of connections with home maintenance organisations. This reflects the agency's strategic focus of developing its own social enterprise service around home maintenance

Furthermore, local working partnerships, funding opportunities and the needs of the local population may impact how agency Caseworkers develop partnerships. For instance, some Chief Officers indicated that they had partnerships with their local health board.

Finally, regional variations in levels of deprivation, rurality and housing quality may influence the breadth of connections that Caseworkers make. For instance, according to the Welsh Index of Multiple derivation (Welsh Government, 2014f) there are pockets of income, housing and health deprivation spread across Wales. These factors have the potential to impact how Caseworkers link with their partner organisations. For instance, in a rural area which has high levels of housing and income deprivation, like parts of North East Wales, Caseworkers may make a greater breadth of connections with a range of organisations such as fuel poverty, slips, trips and falls and health organisations. 
These explanations appear to align with previous social network studies (Coleman et al., 1957, 1966; Van den Bulte \& Lilien, 2001; West et al., 1999) which argue that the context in which networks and relationships are grounded should also be considered. In this thesis client's preferences have been shown to influence the way Caseworkers connect with external organisations to deliver services.

\section{Clients shaping service delivery}

The final factor which was associated with how Caseworkers link with external services was an 'issue on the ground', namely when they encountered clients who were reluctant to change their home environment. There were six types of organisations associated with this issue, and in each instance, correlated with a higher breadth of connections.

These organisations were distinct because client's needs could be addressed without having to make vast changes to the environment i.e. re-enablement, domestic help and fuel poverty organisations. In other words, the services provided by these organisations would be minimally invasive in terms of changing the clients' environment.

This finding is important because it illustrates how links with organisations is shaped not only by the client's needs, but by their preferences and demands.

\section{Factors that shape how often Caseworkers connect with external organisations}

Caseworkers who indicated that they had previous experience of working in managerial roles made more frequent connections with personal support services (e.g. befriending and community support) while making less frequent connections with charities and home maintenance organisations. This could be a result of the social and human capital that these Caseworkers have developed through their past work experience. Managerial roles require 'planning and supervision of operations' (The National Archives, 2016), and this may help them to manage a diverse portfolio of contacts with external organisations.

Furthermore, working in public healthcare services (e.g. NHS) before joining Care \& Repair Cymru was associated with less frequent referrals to health services. Caseworkers with this sort of professional background may have built up effective professional connections so that more frequent contact is not required. These 
findings give an insight into certain management strategies used by Caseworkers to make best use of their capital to minimise the time spent on linking with external organisations.

Finally, 'changing eligibility criteria for benefits claims' is associated with Caseworkers making more frequent contact with personal care services and home maintenance organisations. These results suggest that the current social context of welfare retrenchment also influences how Caseworkers are connecting with external services. Changing eligibility criteria for benefits claims may well result in more frequent contact, because accessing these types of services for their clients requires more negotiation.

As with client's preferences, the social and policy context has influenced the frequency of connections that caseworkers make with external organisations.

\section{Experiences of the Caseworker service: 'emotion works'!}

Qualitative insights provided by clients coming into contact with Caseworkers give an insight that there is something unique in how Caseworkers are linking with them. Most notably, the relationships between Caseworkers and their clients involved a level of emotional engagement or emotion work.

From the Caseworker perspective this meant developing relationships based on empathy, reliability and trust. Client's felt that this led to the formation of genuine caring relationship that alleviated stress and provided them with reassurance. For instance, several clients felt as though Caseworkers took an active interest in them, their family and their overall wellbeing, and reassured them through times of change or stress.

Feelings of trust, reliance and reduction in stress are affective outcomes which stem from the relationships that Caseworkers build and maintain with their clients and involves emotional engagement and going the extra mile. In effect they are taking additional responsibility for clients through instrumental and affective tasks normally undertaken by family members.

The notion that Caseworkers actively care for their clients is illustrated by the strain that frontline workers talked of in the focus groups. Many of the Technical Officers and the Caseworkers described instances where they would encounter difficult 
situations or instances where they were unable to provide solutions for their clients. The findings give the impression that many Care \& Repair Cymru staff remove or alleviate the stress and worry from their clients and in doing so almost take on this stress themselves. However, despite this, many Caseworkers and Technical Officers stated that they derive a lot of job satisfaction from their work roles.

These forms of investment represent the emotion work that Caseworkers undertake to enhance the well-being of their clients. Emotion work is therefore a key element of the Caseworkers' interaction with their clients and shows that they are linking with them in a different way to their other professional contacts. With the exception of providing emotional support (England \& Farkas, 1986; Erickson, 1993, 2005; Zapf et al., 1999), support (Vassilev et al., 2013), and empathy (England \& Farkas, 1986), most theories of emotion work simply refer to the actions or processes undertaken to develop positive relationships and enhance the wellbeing of others (England \& Farkas, 1986; Erickson, 1993, 2005; Strazdins, 2000). This thesis has extended emotion work theory to a new workforce and has provided empirical evidence as to what some of these processes or actions entail for Caseworkers.

Trust has been identified as a key component of the relationships between the Caseworkers and clients. It is necessary for Caseworkers to build relationships with their clients and is also an outcome of the client and Caseworker interaction. Trust is a notion which is referenced by Coleman (1988); Nahapiet and Ghoshal (2000); Putnam (2000); Tsai and Ghoshal (1998) in their work on social capital. In particular, Putnam's iteration of social capital theory suggests that the presence of trust is indicative of the volume of social capital possessed by Caseworkers and Care \& Repair Cymru agencies (Putnam, 2000). The findings show that trust is a pre-requisite and outcome of the links that Caseworkers make with clients.

Therefore, emotion work is a skill that can be developed perhaps through work or possibly it is an extension of an individual's personality. Several members of the Care \& Repair Cymru workforce stated that their Caseworker role is more of a vocation and requires a very specific type of person. Specifically, they need to be individuals who can listen and relate to clients in addition to having the practical skills and knowledge. Taking this notion into consideration, the thesis findings confirm that emotion work not only captures the unique way that Caseworkers link with clients, but it is a manifestation of their social and human capital and lived experiences. 
Caseworkers use their human capital i.e. their skills, educational knowledge and experience to relate and link with their clients and refer them onto other individuals or services that can help them. They also build up trust using these skills in addition to their social capital which allows them to deliver services effectively to their clients to meet their needs and facilitate independent living.

This in part links with what some social capital theorists call charisma which is intrinsic to individuals (Glaeser et al., 2002). Emotion work is also partly innate, but it is also reflective of Caseworkers' professional competencies which stems from their human capital. The findings from this thesis provide illustrations of how emotion work manifests itself in the Caseworker service from the perspective of their clients. In other words, the emotional work undertaken by Caseworkers is complementary to their human capital and is necessary to build relationships.

The interpretation here is of emotion work. Contrary to emotional labour theory, Caseworkers are not simply putting on an affective display to conform to organisational expectations. Instead it appears that the Caseworkers take an active interest in their client's lives and become invested in the outcomes.

\section{Implications of the research for government and Caseworkers in the current policy climate in Wales and beyond}

The results have some implications for the development and delivery of services.

They highlight the potential relevance for a more formal mentoring or training scheme focused on helping Caseworkers create better links with other staff within and beyond their own agencies. This may help to up-skill members of certain agencies or new starter Caseworkers and make the organisation more competitive or specialist in a certain area in a shorter period of time.

The Caseworkers' network shows individuals are linking as hubs of connectivity, bridging agents, and cliques or sub-groups. This has important practical implications; these individuals may well be crucial for spreading information across the Caseworker network. It would be for Care \& Repair Cymru to assess if there is some strategic significance in taking a closer look to the relevance of these hot spots. 
Findings about social and human capital have shown that some forms of expertise can stimulate networking and external linking. For recruitment purposes, if cross sector and inter-agency working remains a priority, these human and social capital profiles can inform recruitment strategies. For instance, being aware of pockets of expertise (e.g. knowledge of benefits funding applications, sensory impairment adaptations and organisations willing to fund or collaborate with agencies) could lead to the recruitment of individuals who have different or complementary knowledge and skill sets to each agency.

Identifying the highly skilled or connected individuals in the network could also have important implications for future training plans. Agencies may wish to upskill the rest of their Caseworker workforce so that if a highly skilled Caseworker were to leave then the $\mathrm{CW}$ to $\mathrm{CW}$ network could adapt and be able to retain a good range of knowledge and skills so that they could continue to meet the needs of their clients. Furthermore, agencies may wish to encourage greater links between Caseworkers, so if individuals are highly connected or act as bridges in the CW to CW network were to leave, then the network.

This thesis has added to the knowledge and research record on frontline workers and service delivery which have been examined through social network analysis. In a similar manner to Woodcraft (2011), the findings of this thesis has found that individuals occupy different roles in social networks. This has reinforced the importance of recognising different networking roles as this can help to improve service delivery.

At an organisational level, Care \& Repair Cymru recognise the importance of emotion work and building affective relationships with clients. This is also supported in part through the socio-demographic data from the Caseworkers, where over half of them (56.4\%) had attended interpersonal skills training including mental health and dementia training. Further investment into training of this nature may help Caseworkers develop their awareness and emotional skills as findings show this to be a key element of successful relationship building.

Additionally, while the Caseworkers' emotion work is valued, it has to be acknowledged that it can put them at risk of emotional burnout. This has been acknowledged and explored in health care professions e.g. nursing (Zapf et al., 1999) and represents an important aspect of human resource management. 
Again, at an organisational level, the findings could be used by Chief Officers of the $\mathrm{C} \& \mathrm{RC}$ agencies to extend their awareness of the cross-collaborative nature of their workforce and the reality of the Caseworker role. During initial introductions to Care \& Repair Cymru agencies it was apparent that each agency held an amount of pride about their agencies and viewed them to be autonomous and independent from each other. However, the findings question this assumption and indicate that inter-agency collaboration takes place. Therefore, the perceptions held by the Chief Officers and the workforces they manage could be changed to greater appreciate the collaborative practices that takes place between agencies.

The Caseworkers can use the findings to develop a greater awareness of how their work fits into the broader national level strategies for older people. While many of the Caseworkers indicated that they work in collaboration with their Caseworker colleagues and organisations beyond Care \& Repair Cymru, many may not appreciate the implications of this and how they operationalise core policy values of the Social Services and Well-being(Wales) Act 2014 and the Well-being of Future Generations (Wales) Act 2015.

The findings also have several policy implications. For instance, this thesis addresses knowledge gaps about a diverse and specialised CW workforce who deliver services with the aim of facilitating independent living for older people in a context of austerity and collaboration. The findings also point out the potential oversights of the Auditor General for Wales (2018) report and can be used by Care \& Repair Cymru as an evidence base to illustrate that cross-collaborative working is a key feature of the $\mathrm{C} \& \mathrm{RC}$ agencies as a whole.

Additionally, this thesis presents Care \& Repair Cymru Caseworkers as an example of good practice of collaborative working across different sectors also has implications for the rest of the UK and beyond. The findings can be used as a strong working example of effective partnership working in practice, which other organisations, sectors, and regions could seek to replicate. This is particularly poignant in a policy context of austerity and increased competition.

This thesis has further implications for policy because it points to the complexity associated with attempting to facilitate independent living and ageing in place for older people. This is exemplified by the finding that Caseworkers have identified that 
they link with a total of 383 organisations across 16 different sectors. This indicated that the Caseworkers' work does not simply entail addressing housing needs, rather, Caseworkers attempt to address a range of issues including health, social care and financial assistance to help their clients retain their independence and age in place. Additionally, the importance placed on building relationships with clients has been highlighted in the qualitative data. However, the value of the Caseworker service is also highlighted because they use their skills and knowledge and collaborative practices to address this myriad of issues. This operationalises with the policy agenda of collaboration and person-centred service delivery.

\section{Theoretical contributions}

This thesis has made several contributions to knowledge and has added to the theoretical record of frontline workers such as carers, support workers and Caseworkers who work with older people across health, housing and social care sector. Additionally, a social network analysis approach has also been applied to the Welsh context and a third sector organisation. This approach to research has not previously been undertaken in Wales and offers several theoretical contributions.

An example of previous research on frontline public service delivery was a study by Woodcraft (2011),who SNA to explore and improve public service delivery in a high priority neighbourhood. This study found that a lack of trust in public services was associated with apathy and low levels of networking between residents. Woodcraft did not explore trust theoretically, but rather used this finding to shape the practical initiatives that were used to improve public service delivery. However, this thesis has developed on this by examining trust theoretically as a manifestation and consequence of social and human capital.

This thesis has achieved its objective by examining the individuals who comprise a workforce that deliver services to older people. Social and human capital have been used to determine the characteristics or attributes that the Caseworkers as individuals bring to Care \& Repair Cymru. This has confirmed the contention made by Ennis and West $(2010,2013)$ that a social network analysis approach can provide a greater understanding of the assets contained within a community or group of individuals which can then be used to inform community work and development practices. Specifically, understanding how micro-level relationships can be used to challenge broader social structures or issues can be explored. 
The findings have contributed to knowledge of applied social network studies by confirming that the assets or characteristics contained within a group of individuals (notably previous experience of working in the business sector and the length of time CWs have been in post) have tangible outcomes and impacts in terms the numbers of contacts CWs develop beyond their own organisation.

Like Nicaise et al. (2012) this thesis has explored networking in the context of fragmented model of public services. Whilst Nicaise et al. (2012) studied several organisations and their networks across two settings and this thesis focused on one organisation; both identified the importance of people or organisations acting as brokers as part of service delivery as they have the capacity to straddle and bring together fragmented services and link individuals to several different types of organisation.

Both Bolton (2000) and Lopez (2006) explored nursing and care work workforces using emotional labour and both concluded the applicability of this theory to such occupations was limited and needed reconceptualising. This is because workers in these professions do not simply not employ surface or deep acting when engaging with patients or clients and hence emotional labour theory does not accurately capture or describe the nature of emotional exchanges in caring professions. This position was reinforced by the qualitative findings of this thesis that indicated that Caseworkers take time to form a genuine relationship with their clients.

Bolton (2001) also used the theory of emotion work to examine how nurses cope with the emotional demands of their work. However, very few studies have explored the emotional processes themselves outside of creating a space for them to occur (Lopez, 2006). This thesis has addressed this gap by exploring the processes that enable Caseworkers to build relationships with their clients. The findings have added to knowledge by providing examples of what these processes entail including emotional engagement, taking an interest in the lives of their clients and completing tasks which are traditionally undertaken by family members.

Furthermore, previous research by Bolton $(2001,2005)$ has also explored the strain or burn out that can occur as a consequence of emotions in the workplace. The qualitative data from the Caseworker and Technical Officer focus groups provides evidence for this and reaffirms the potential for burnout and strain to occur as a result of emotion work. Whilst emotional labour suggests that burnout occurs from the 
need to continually align emotional displays to meet with organisational expectations (Hochschild, 2003), the findings from this thesis provides evidence that that strain and emotional burnout can occur as a result of workers' genuine emotional engagement and investments made to establish relationships with clients.

Finally, very few studies have investigated how emotional processes are used to develop relationships between workforces and service users. This thesis has addressed this gap and has added to the theoretical literature on emotions in work forces by applying emotion work to explore how Caseworkers build and maintain their relationships with their clients.

In sum, social network analysis, network, capital and emotion work theory has been applied to a new context and workforce which has led to some fascinating insights on collaborative practices, trust, relationship building and emotional engagement, which has contributed on knowledge in several ways.

\section{Are Caseworkers link agents?}

The empirical evidence gathered in this thesis has meant that the preliminary conceptualisation of the Caseworkers as potential link agents outlined in Chapter Two can be revisited. The evidence shows that Caseworkers do act as link agents by interacting with different groups of people in different ways to deliver services. However, the actions that they undertake go beyond case management and brokerage which are not captured in their job description.

As part of their role they interact with other Caseworkers, to share knowledge, expertise and support. This can be achieved through direct connections with other individuals or groups of Caseworkers, or by acting as a bridge between colleagues who are otherwise unconnected. Additionally, CWs link with a diverse range of organisations outside of Care \& Repair Cymru. The breadth and frequency of these connections can be influenced by a Caseworker's capital or driven by contextual factors including the clients' preferences as well as their needs. In some instances, they do act in a brokerage capacity by referring clients to their partner organisations e.g. contractors. The final and perhaps most obvious type of link that the Caseworkers make are with their clients. CWs form genuine caring relationships with them using emotion work, which is a manifestation of the capital that they have accumulated. 
Figure 8.1- Illustration of the conceptualisation of the Caseworkers as link agents

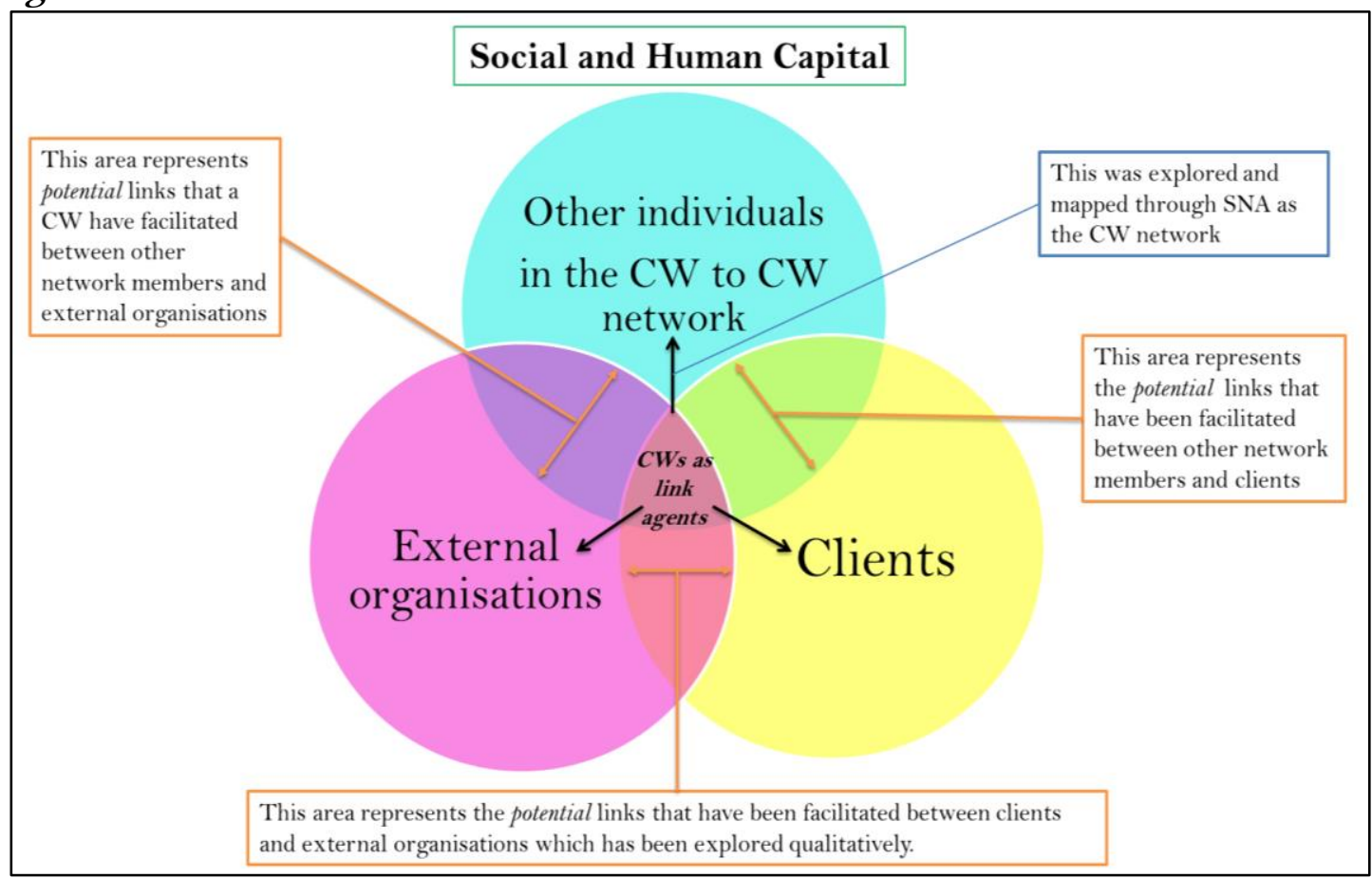

An illustration of how the social and human capital of Caseworkers, or the sum of their competencies, shapes how they link with clients, external organisations and other Caseworkers is provided above (see Figure 8.1).

Figure 8.1 captures how Caseworkers link with others and the potential that these links have to put otherwise unconnected individuals together e.g. clients and services. The links Caseworkers develop with colleagues, external organisations and clients has been confirmed and explored in this thesis. And the patterns of networking and links with external organisations are shaped in some part by the capital that CWs have developed.

However, the results and Figure 8.1 show that there are other potential links and networks that Caseworkers are involved in. Specifically, they may link other colleagues with external organisations; clients with external organisations; and other Caseworkers and clients. These represent potential networks which could be explored.

It is also important to note that Caseworkers' emotion work is not just the skills that they employ to deliver services to clients. Rather, emotion work encompasses much more than just skills. In this thesis it captures the care that Caseworkers bring to their role, which is reflected in them going the extra mile by carrying out instrumental and affective tasks to help their clients as much as possible. At times it appears that 
Caseworkers are not merely striving to achieve their organisational aim but are filling a gap of informal care; a role has traditionally been fulfilled by the family. These actions also have the potential to have a detrimental impact on CWs.

\section{Limitations}

While this thesis has produced several innovative findings, there are limitations.

First is that the study design did not examine relationships between Caseworkers and other staff members at Care \& Repair Cymru. However, the qualitative data also reveals that collaboration and interaction between Caseworkers and Technical Officers within the agencies was also important. The thesis presents a partial picture of the structure of relationships in which Caseworkers are engaged with in the organisation as a whole. However, undertaking this more extensive analysis of the social networks would be impossible in the context of a PhD.

Second, the data captured in the online self-completion questionnaire only provides a snapshot in time of the Caseworker network. This is particularly relevant for this thesis because at the time of data collection Care \& Repair Cymru agencies were undergoing a mass restructuring, which could impact how Caseworkers link with each other and external organisations.

Also, due to the restructuring of the Care \& Repair Cymru agencies, in some instances there were Chief Officers who were not familiar with all the counties that their merged agencies covered. Therefore, their contextual knowledge of the context in which their CWs work was limited.

Additionally, bias may have arisen when the social network data was collected as Caseworkers may not have thought to include Caseworker colleagues from their own agency. Therefore, the sociogram may not capture all of the links that the participating Caseworkers have developed.

Another limitation of the thesis comes from the study design, and in particular, the recruitment of the client participants. Because Care \& Repair Cymru agencies were involved in their selection, there is a potential that they only selected people who they believed would be satisfied with the services, or who they thought would be the most articulate. 
The design of the questions in the self-completion questionnaire could be improved in future studies. Two questions in particular could have been designed in a way to capture more information about the Caseworkers. These include the questions pertaining to the age of the participants and how long they had been in post as a Caseworker. The responses were already coded into categories, however for the purposes of analyses it would have been more beneficial to collect continuous variables and not categorical ones.

Finally, the small sample sizes used were a limitation. Certain statistical tests could not be undertaken because there were not enough individuals. For instance, the Latent Profile Analysis may have detected a greater diversity of networking profiles had the sample size been bigger.

\section{Critical outlook for further research}

This research has produced innovative findings which contribute to both academic and practical knowledge and the results have highlighted several potential areas where further research can be carried out.

First research could be conducted on the Care \& Repair Cymru Caseworker population. This would create a longitudinal study which examines how the networking activity and inter-organisational working of Care \& Repair Cymru Caseworkers changes over time. Re-mapping the CW to CW network over time is particularly important because of the current social context where resource allocation needs to be justified.

Second, there is potential to expand the social network analysis approach in current research to include all Care \& Repair Cymru staff and the external organisations they link with. This allows for a fuller and more detailed exploration of all the potential networks, links and networking role that Caseworkers are involved in as highlighted in Figure 8.1.

Third, the regional variation in networking and linking with external organisations could be explored further to determine if indicators such as the Welsh Index of Multiple Deprivation could be included as a proxy for variations in health, housing and financial wellbeing. 
Additionally, the SNA approach used as part of this thesis can also be applied to the wider UK contexts and beyond. For instance, in England there have been many conversations and debates (including Age UK, 2013; Glasby, 2017; Goodwin, 2012; Ham, 2014; RAND Europe \& Ernst \& Young, 2012) about whether health and social care budgets should be merged to produce more effective and efficient care packages for older people (amongst others). Using a social network analysis approach would enable researchers to map where collaborative practices are occurring and instances where they are not. These findings could then be used to inform policy and practice.

\section{Conclusions}

Using a pragmatic exploratory mixed-methods approach this thesis has shown that Caseworkers are acting as link agents- working collaboratively with their fellow Caseworkers, external organisations and building affective relationships with their clients as part of delivering services to facilitate ageing in place and independent living.

The networking that occurs between colleagues shows that there is specialist expertise spread throughout the Caseworker network. This an important finding for Care \& Repair Cymru because these individuals have key skills or knowledge which can be drawn upon to continue to meet the needs of an increasingly older population in the face of welfare retrenchment, austerity, increasing complexity of needs and diminishing pools of informal support.

The links that they make with external organisations show that as an organisation Care \& Repair Cymru is meeting the aims of the Wellbeing and Future Generations (Wales) Act 2015, most notably the collaboration agenda. Not only are Caseworkers making a lot of connections with external organisations but the organisations that they link with are varied. Linking with these organisations demonstrates that Caseworkers are addressing multiple requirements which go well beyond housing. This research has effectively provided an evidence base which the Auditor General for Wales (2018) was lacking. For instance, the report claimed that public agencies that deliver housing adaptation services and solutions lack strategic links to other organisations; and ignore the wider needs of older people by focusing mainly on the structural housing needs. This thesis has proved that this is incorrect.

Caseworkers also build relationships with their clients by going the extra mile to take on instrumental and affective responsibilities which are traditionally associated with 
kinship networks. While as an organisation Care \& Repair Cymru recognise the importance of this aspect of the Caseworkers' role, it vital for policy to acknowledge this otherwise it can be glossed over or lost in a context where policy agendas are focused on efficiency, and the monetary value of publicly funded services.

In sum, the Caseworker role is vital in the grass roots level implementation of Welsh Government's wider social policy, social care and economic agendas by promoting citizenship-focused service delivery, cross-sector partnership working, and stimulation of local businesses (Auditor General for Wales, 2018; Care \& Repair Cymru, 2017b; Expert Group on Housing an Ageing Population in Wales, 2017; Welsh Government, 2014b), but this largely remains unrecognised. In effect they are a hidden workforce operating in the background facilitating cross-sector working and service delivery to aid older people to live independently and age in place.

They are indeed Wales' best kept secret! 


\section{Appendices}

\section{Appendix Item 1- Illustration of the ontological and}

epistemological underpinnings of this project

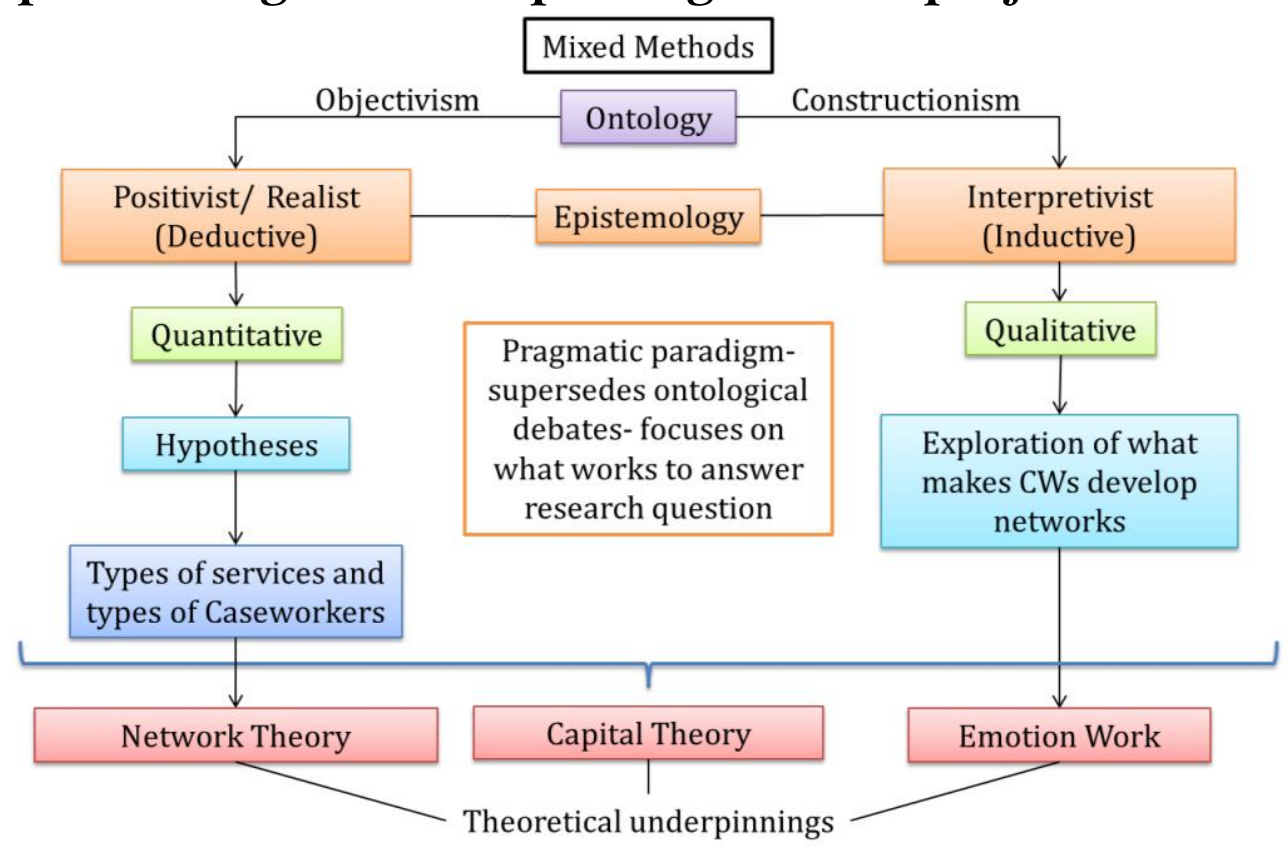


Appendix Item 2: Information Pack for Care \& Repair Cymru Caseworkers

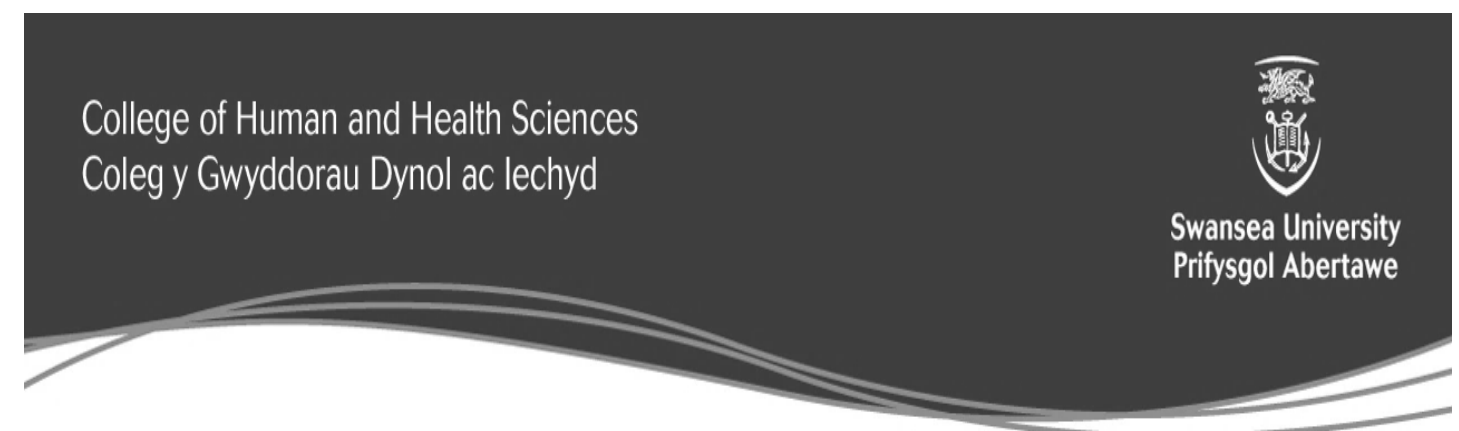

I am pleased to invite you to take part in my research study exploring the role that Care \& Repair Cymru Caseworkers have in helping older people retain their independence so that they can live in their own homes and communities. I am particularly interested in exploring how Caseworkers act as brokerage or link agents in helping to meet the client's requirements and needs.

However, before you decide if you would like to participate, it is important for you to understand why the research is being done and what participation will involve.

Please take some time to read the following information carefully, and if you would like some further information or if anything remains unclear please feel free to get in contact using the information listed at the end of this form.

I would like to stress that you do not have to accept this invitation and should only agree to take part if you want to.

Thank you for taking the time to read this.

\section{This form is available in large print. If you require large font, please contact:}

\section{Joanna L. Wolton, Centre for Innovative Ageing, College of Human and Health Sciences,

\author{
University, \\ Singleton Park, Swansea, SA2 8PP.
}

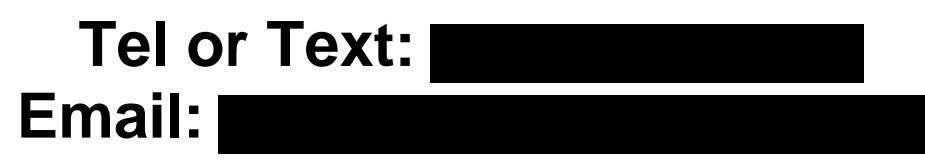




\section{What is the purpose of the study?}

Enabling older people to remain in their own homes and communities for as long as possible is an issue that is currently high on the agenda of the Welsh Government, and funding has been made available to recognise this goal. Care \& Repair Cymru are one of the organisations working with the Welsh Government to achieve this.

The aim of this study is to explore the role that Care \& Repair Cymru's Caseworkers play in helping older people retain their independence and live in their own homes and communities. In particular the researcher will be focusing on understanding how Caseworkers act as brokerage or link agents between clients and other organisations in helping to meet the client's needs and requirements. This study will aim to map the connections made by Caseworkers across Wales and discuss their networking activities to demonstrate partnership working within the local and national social context. Additionally, this research is interested in the views and experiences of the older people as clients of Care \& Repair agencies.

My research will provide more evidence about the contributions that Care \& Repair agencies across Wales make in helping older people to remain living in their own homes and communities for as long as possible. This research will also contribute to the evidence-base needed to ensure that policies and funding to help support these services to continue their work into the future.

\section{Why have I been chosen to take part?}

You have been approached because you currently work for Care \& Repair Cymru in the role of Caseworker. Your knowledge of the demands for services in your local area will help the researcher to fully understand the Caseworker role, and how the services offered by Care \& Repair agencies are shaped by the local area.

\section{Do I have to take part?}

You have no obligation to take part in this research. Participation is entirely voluntary, and you are free to withdraw at any time, without explanation and without being disadvantaged in anyway.

\section{What will happen if I decide to take part?}

If you feel satisfied that you have been given enough information about the study and would like to take part, you will be asked to sign the attached consent form and send it back to me in the post at the address listed below or you can send a signed copy by email as a scanned document within a week of receiving this pack.

\section{What will happen if I take part?}

If you decide to take part and return the consent form at the end of this pack back to me, I will then send you a link to the online questionnaire to complete. The questions in the questionnaire will focus on your actions as a link agent and will seek to explore the organisations that you collaborate with to achieve Care \& Repair Cymru's goals of helping older people to retain their independence and remain living in their own homes for as long as possible. 
Once the questionnaires have been completed, I may contact you again via telephone or email to ensure that all data has been correctly recorded. You may also be invited to take part in a follow-up focus group. The follow-up focus group will discuss some of the issues or themes that have arisen in the questionnaire. Please be assured that all personal information that is collected about you will be kept confidential. Your answers may be then be used as points of discussion in a focus group, but no identifying information will be given to participants

Each session will be audio recorded, but your responses will be kept confidential and any results that are published will be anonymous. It is important to note that this research is being undertaken on behalf of Swansea University and not Care \& Repair Cymru.

Please note that this is an independent research project and therefore it is not a form of evaluation and your involvement in the research project will not impact your position in Care \& Repair Cymru.

6. Why is the focus group tape-recorded?

The focus group will be audio-recorded so that I do not miss anything you say. The recordings will be written up and anonymised so that there are no identifying names in the record. The recording will be securely stored by Swansea University and encrypted.

\section{Expenses and/or payment?}

There will be no expenses or payment for your involvement in this project.

\section{Are there any risks in taking part in the research?}

There are no risks involved in taking part, but it is possible you may not want to answer some of the questions. If you do not want to answer any of the questions you do not have to, please just tell me that you would prefer to not answer that particular question and I will move onto the next question.

9. Are there any benefits in taking part in the research project?

There are no direct benefits for the individuals who participate in the study. I hope that the results will provide more evidence about the contributions that Care \& Repair agencies across Wales are making to this policy agency by helping older people to remain independent. The research will also contribute to the evidencebase needed to ensure that policies and funding to help support these services continue into the future.

10. Will my participation be kept confidential?

All information that is collected about you for the study will be kept confidential. I will not include your name or any other information that might identify you in the written record of the interview. The only circumstances in which I would let anyone know you are taking part would be if I felt you may be at risk of harming yourself or someone else.

11. Will my taking part be covered by an insurance scheme?

Those who participate in this ethically approved study have insurance cover provided by Swansea University.

12. What will happen to the results of the study? 
Anonymised data will be stored on a secure, password protected computer for me to analyse during the course of the study. I will not use your name or any other identifying information in the research thesis.

This data will be kept by the researcher beyond the end of the duration of the research project (for approximately five years) and will be used by the researcher and members of the supervisory team to present findings at conferences, on our website and in journal articles. Therefore, the findings of the research will be publicly available.

If you are interested in receiving a copy of the results in a form of executive summary or as a full thesis then the researcher can send you a copy of the findings upon the completion of the study. Additionally, information bulletins about the research will be made through the Care \& Repair website and twitter feeds.

\section{What will happen if I decide I no longer want to take part?}

Participation is entirely voluntary, and you can withdraw from the study at any time without explanation. Results that are already written up at the time of withdrawal may still be used, only if you are happy for this to occur. Otherwise, you may request that they are destroyed and that no further use is made of them. Your decision will not count against you or disadvantage you in any way.

\section{Who can I contact if I have further questions?}

You can call, email or write to me with any concerns or questions using my contact details on the next page. Please ask questions as often as you want. I am available to take telephone calls Monday-Friday between 9.30am and 3.30pm. You can also contact my supervisor, Dr Sarah Hillcoat-Nallétamby.

Joanna Wolton
PhD Student and Researcher
College of Human and Health
Sciences,
Centre for Innovative Ageing
Floor 1, Haldane Building,
Swansea University
Swansea
SA2 8PP
Tel
Email:

Dr Sarah Hillcoat-Nallétamby Associate Professor of Social Policy and Ageing.

College of Human and Health Sciences,

Centre for Innovative Ageing

Swansea University

Swansea

SA2 8PP

Tel:

Email: 
Consent Form

This is a requirement of the College of Human and Health Science's (based at Swansea University) ethics procedure. The researcher is required to seek informed consent of all participants. This is not a requirement on behalf of Care \& Repair Cymru and will not affect your position or relationship to Care \& Repair.

Name:

Location of Care \& Repair Agency:

Office telephone number:

Mobile:

Email:

1. I confirm that I have read and understood the participant information sheet attached to this form and agree to take part in the above research.

(Please tick and initial).

2. I understand what my role will be in this research project, and all my questions have been answered to my satisfaction by the researcher.

므 (Please tick and initial).

3. I understand that my right to withdraw from the research at any time, for any reason and without prejudice.

(Please tick and initial).

4. I have been informed that any information I provide will be anonymised and that the confidentiality of the information will be safeguarded.

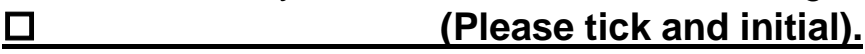

5. I consent to the interview/ focus group being audio recorded for research purposes.

ㅁlease tick and initial).

6. I understand that I am free to ask any questions at any time before and during the study

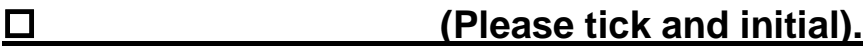

7. I have been provided with a copy of this form and the participant information sheet.

(Please tick and initial).

Data protection: I agree to the University processing personal data that I have supplied. I agree to the processing of such data for any purposes connected with the Research Project as outlined to me. 
Witnessed by:

(Print) Signed: Date:

This form is available in large print. If you require large font, please contact

Joanna L. Wolton, Centre for Innovative Ageing, College of Human and Health Sciences, University,
Singleton Park, Swansea, SA2 8PP. Tel or Text: Email: 


\section{Appendix Item 3: The Self-completion Caseworker Questionnaire}

College of Human and Health Sciences

Coleg y Gwyddorau Dynol ac lechyd

Swansea University

Prifysgol Abertawe

\section{Caseworker Questionnaire}

This questionnaire is part of a research project being carried out by Swansea University, which aims to provide a better understanding about the important role that caseworkers play in helping older people to live independently at home in the community, by linking and working with other service providers and organisations on behalf of their clients. We would be very grateful if you would take time to complete the questions below, as your answers will help us develop a clearer picture about the caseworker role across the different Care \& Repair agencies in Wales. You will need about 30 minutes to complete the questionnaire.

As explained in the information pack you will have received about the research, all your answers will be treated in the strictest confidence. This is an independent research project being carried out by Swansea University, and in compliance with the University's regulations, when analysing or reporting our findings we will not include your name or any information that might identify you personally. Some of the information gathered may subsequently be used to develop new questions for follow-up focus group discussions with Care \& Repair caseworkers. For reasons of quality control and confidentiality, we would ask you not to discuss your responses to the questionnaire with your colleagues. Your opinions are important to us- Thank you for participating in this research! 


\section{Section A- Your background}

This part of the questionnaire will help us have a better understanding about caseworkers in terms of their educational qualifications, training and past employment history. Please tick the appropriate boxes which best describe your situation and profile.

1. In which Care \& Repair Agency are you employed?

2. Gender:

Male

Female

3. Age: 16-19

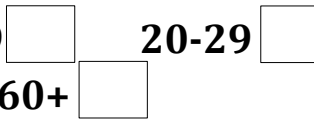

30-39

40-49

50-59

4. What is your highest level of educational qualification?
a) No Qualifications
b) GCSE/ O Levels or equivalent
c) Apprenticeship
d) Further education/ A Levels or Equivalent
e) Higher education/
f) Other degree level or above

If "Other" please specify in the box below and on the next page:

5. Do you have any other professional or vocational qualification?

Yes No

If "No" please skip to Question 6. If "Yes", please specify in the box below:

6. Please let us know about any training you have received since working for Care \& Repair in your role as caseworker, including any certified qualifications (e.g. First Aid)? Please specify in the box below (including approximate date of completion: 
7. Please could you explain how you think this training has helped enhance your work:

8. Please indicate which sectors you have previously worked in prior to taking on the role of caseworker (Please tick all relevant categories)

Banking \& finance

$\square$ Business

Construction/

engineering

Charity \& voluntary sector

$\square$ Creative arts \& $\square$ Education
design

Environment/agriculture \begin{tabular}{|}
$\square$ Healthcare (non- \\
NHS)
\end{tabular} Hospitality, leisure,
travel

IT \& Technology

$\square$ Legal

Logistics \& transport

Marketing, media, PR

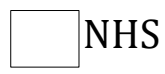

$\square$ Public
administration

(e.g. local

Government)

$\square$ Publishing/ journalism

$\square$ Recruitment \& HR

Retail/ customer service

Security/uniformed services (e.g./ HM

Social services

Volunteer

Forces/Police)

None

Other

Other please specify in the box below 
9. Indicate which of the following categories describe your past work experience. (Please tick all categories that are relevant to you).

a) Professional occupation (manager/ director/ CEO)

(e.g. science, engineering, technology, conservation, health, education, Legal, IT, public or private sector services etc.).

b) Associate professional and technical occupation

(e.g. technicians in science, engineering, IT, nursing, therapy, social welfare, Police, fire \& ambulance, sports and leisure services).

c) Administrative and secretarial occupation

(e.g. civil service, clerk, library assistant, IT engineer, agricultural \& related trades, market research interviewer, communication operator, secretary, Personal assistant).

d) Skilled trade occupation

(e.g. bricklayer, roofer, carpenter, plasterer, painter, IT engineer, agricultural $\&$

Related trades, butcher, baker, chefs, florist etc.)

e) Personal service occupation

(e.g. care or nursery assistant, child minder, veterinary nurse, barbers,)

\section{f) Sales and customer service occupation}

(e.g. sales assistants, market/street traders, call centre agent, Hospitality, travel).

g) Process, plant and machine operative (e.g., routine inspectors, HGV drivers, bus, coach or agricultural machinery Drivers etc.)

h) Elementary occupations

(E.g. general labouring, cleaners, catering assistants/bar staff, traffic wardens).

Other, please specify in the box below: 
10. Please tell us how you think your previous work experience has helped in your role as a caseworker (e.g. experience of working with the public/ knowledge of benefits applications). Please could you explain using the box provided below: 


\section{Section B-Your role and responsibilities as a caseworker}

This part of the questionnaire is designed to help us have a better understanding of the different and potentially complex aspects of your work in your role as a caseworker. Please complete this section by tick the appropriate boxes

11. What is your job title (i.e. caseworker/technical caseworker)?

12. Is your role funded by 'core funding'?

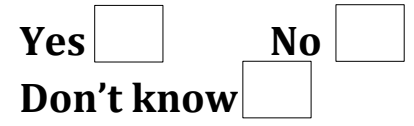

12a. If 'Yes' or 'Don't know' please skip onto the next question. If 'No', please indicate how your post is funded in the space provided below:

13. How long have you been a caseworker?

Less than 6 months

6 months- 1 year

1-2 years

2-3 years

3-4 years

4-5 years

$5+$ years

14. Do you work full time or part time?

Full time

Part-time

15. Do you work across several Welsh counties or parts of a county Yes No

15a. If no, is there a specific area in which you are based? If so, please let us know in the space provided below: 
16. What would you say are the main tasks that you undertake as part of your role as a caseworker?

a) Basic benefits check

b) Informing the client about their rights and entitlements

c) Fundraising on behalf of clients to pay for works needed

d) Making referrals to other services, (e.g. re- enablement)

e) Linking with services across Different services/sectors/ providers

f) Introducing a client to digital Techology (e.g.RNIB Online Today)

g) Up-dating CARIS database

h) Healthy homes checks

i) Case management

j) Linking with other family members

k) Providing advice \& information about housing options (e.g. moving home, adaptations)

m) Promoting Care and Repair and the services they provide to clients, organisations and groups.

l) Linking with other Care \& Repair agencies and staff

n) Supporting a client through a Period of change e.g. moving on/ building works/ hoarding

Other, please detail in the space provided below: 


\section{Section C- Linking with other Caseworkers}

In this section we are interested in understanding more about the networks you have built up with other caseworker colleagues who may be based wither in your own, or in other Care \& Repair agencies. The different Care \& Repair agencies are listed below. Please provide the names of any caseworkers in any of the agencies that you have contact with for work purposes. If you do not contact any caseworkers from a particular agency, simply leave the box blank. [If you are in contact with more than one caseworker, please ensure you separate each name with a comma (,)].

17.

\section{a). Blaenau Gwent Care \& Repair:}

\begin{tabular}{lll}
1. & 2. & 3. \\
\hline 4. & 5. & 6. \\
\hline 7. & 8. & 9. \\
\hline
\end{tabular}

\section{b). Bridgend County Care \& Repair:}

\begin{tabular}{lll}
\hline 1. & 2. & 3. \\
\hline 4. & 5. & 6. \\
\hline 7. & 8. & 9. \\
\hline
\end{tabular}

c). Care \& Repair Caerphilly:

\begin{tabular}{lll}
\hline 1. & 2. & 3. \\
\hline 4. & 5. & 6. \\
\hline 7. & 8. & 9. \\
\hline
\end{tabular}

\section{d). Cardiff \& the Vale Care \& Repair}

\begin{tabular}{lll}
\hline 1. & 2. & 3. \\
\hline 4. & 5. & 6. \\
\hline 7. & 8. & 9. \\
\hline
\end{tabular}

\section{e). Care \& Repair Carmarthenshire LTD:}

\begin{tabular}{lll}
1. & 2. & 3. \\
\hline 4. & 5. & 6. \\
\hline 7. & 8. & 9. \\
\hline
\end{tabular}

\section{f). Conwy and Denbighshire Care \& Repair}

\begin{tabular}{lll}
\hline 1. & 2. & 3. \\
\hline 4. & 5. & 6. \\
\hline 7. & 8. & 9. \\
\hline
\end{tabular}

\section{g). Gofal A Thrwsio Gwynedd A Môn:}

\begin{tabular}{lll}
1. & 2. & 3. \\
\hline 4. & 5. & 6. \\
7. & 8. & 9.
\end{tabular}

\section{h). Merthyr Care \& Repair:}

1.

2.

3. 


\begin{tabular}{lll}
\hline 4. & 5. & 6. \\
\hline 7. & 8. & 9. \\
\hline
\end{tabular}

i). Care \& Repair Monmouthshire and Torfaen

\begin{tabular}{lll}
1. & 2. & 3. \\
\hline 4. & 5. & 6. \\
\hline 7. & 8. & 9.
\end{tabular}

i). Neath Port Talbot Care \& Repair:

\begin{tabular}{lll}
1. & 2. & 3. \\
\hline 4. & 5. & 6. \\
7. & 8. & 9. \\
\hline
\end{tabular}

k). Newport Care \& Repair:

\begin{tabular}{lll}
1. & 2. & 3. \\
\hline 4. & 5. & 6. \\
\hline 7. & 8. & 9.
\end{tabular}

1). Care \& Repair North East Wales (Flintshire and Wrexham)

\begin{tabular}{lll}
1. & 2. & 3. \\
\hline 4. & 5. & 6. \\
7. & 8. & 9. \\
\hline
\end{tabular}

m). Care \& Repair in Powys:

\begin{tabular}{lll}
1. & 2. & 3. \\
\hline 4. & 5. & 6. \\
\hline 7. & 8. & 9. \\
\hline
\end{tabular}

n). Care \& Repair Rhondda Cynon Tâf:

\begin{tabular}{lll}
1. & 2. & 3. \\
\hline 4. & 5. & 6. \\
\hline 7. & 8. & 9. \\
\hline
\end{tabular}

o) Swansea Care \& Repair:

\begin{tabular}{lll}
1. & 2. & 3. \\
\hline 4. & 5. & 6. \\
\hline 7. & 8. & 9. \\
\hline
\end{tabular}

West Wales Care \& Repair (Pembrokeshire and Ceredigion):

\begin{tabular}{lll}
1. & 2. & 3. \\
\hline 4. & 5. & 6. \\
7. & 8. & 9. \\
\hline
\end{tabular}

End of section C 


\section{Section D-Who do you link with?}

A lot of the work caseworkers undertake involves building networks and collaborative links with other service providers, sectors and organisations to ensure that client requirements are met. This section of the questionnaire is designed to help build a clearer picture of the networks you have developed since working with Care \& Repair.

The following tables we would like you to complete relate to different aspects of the caseworker role; (financial applications; fundraising; referrals; healthy home checks; home maintenance/ adaptations). For each table, please indicate which organisations and service providers you link with in your work, and on average how often you contact them.

Please fill in the tables within the five sections using a tick symbol " $\checkmark$ " to indicate which organisations and service providers you approach or collaborate with to help deliver each type of service. If we have missed some, please add them in the spaces at the bottom of the tables labelled "Other" and detail the name and type of service they offer.

We also need to have an idea of how often you link with these different organisations and service providers, so please used a tick symbol " $\checkmark$ " to indicate how often you are in contact with them on an average month.

Finally, we would also like to know if you think that the particular service you link with offer a good standard of service, therefore depending on your opinion indicate either " $\mathrm{Y}$ " for yes or "N" for no.

Please see the example:

(a) Helping client with financial support needs

\begin{tabular}{|c|c|c|c|c|c|c|}
\hline \multicolumn{7}{|c|}{$\begin{array}{l}\text { Applying for financial help: includes organisations that you approach } \\
\text { or refer clients to for support with formal applications for financial } \\
\text { help (e.g. benefits), after a basic benefits check has been completed by } \\
\text { Care \& Repair. }\end{array}$} \\
\hline \multirow[t]{2}{*}{$\begin{array}{l}\text { Organisation } \\
\text { name and logo }\end{array}$} & \multirow{2}{*}{$\begin{array}{l}\text { Contact or } \\
\text { collaboration } \\
\text { made (tick if } \\
\text { applicable) }\end{array}$} & \multicolumn{4}{|c|}{$\begin{array}{l}\text { In an average month how often } \\
\text { would you link with these services } \\
\text { (tick most relevant category) }\end{array}$} & \multirow{2}{*}{$\begin{array}{l}\text { In your } \\
\text { opinion } \\
\text { is this a } \\
\text { good } \\
\text { service } \\
(\mathrm{Y} / \mathrm{N}) ?\end{array}$} \\
\hline & & Daily & Weekly & Monthly & $\begin{array}{l}\text { One-of } \\
\text { contact }\end{array}$ & \\
\hline $\begin{array}{l}\text { Department For } \\
\text { Work \& Pensions }\end{array}$ & $\checkmark$ & & $\checkmark$ & & & $Y$ \\
\hline
\end{tabular}

Please note that organisations can appear in more than one category if they provide more than one service. 


\section{(a) Helping Client with financial support needs}

18.0rganisations you approach after completing a basic benefits check, because they offer support to clients who need help in applying for financial help (e.g. benefits).

\begin{tabular}{|c|c|c|c|c|c|c|}
\hline \multirow[t]{2}{*}{$\begin{array}{l}\text { Organisation } \\
\text { name and logo }\end{array}$} & \multirow{2}{*}{$\begin{array}{l}\text { Contact or } \\
\text { collaboratio } \\
\mathrm{n} \text { made (tick } \\
\text { if } \\
\text { applicable) }\end{array}$} & \multicolumn{4}{|c|}{$\begin{array}{l}\text { In an average month how often } \\
\text { would you link with these services } \\
\text { (tick most relevant category) }\end{array}$} & \multirow{2}{*}{$\begin{array}{l}\text { In your } \\
\text { opinio } \\
\mathrm{n} \text { is } \\
\text { this a } \\
\text { good } \\
\text { service } \\
(\mathrm{Y} / \mathrm{N}) ?\end{array}$} \\
\hline & & $\begin{array}{l}\text { Dail } \\
\mathbf{y}\end{array}$ & $\begin{array}{l}\text { Weekl } \\
\text { y }\end{array}$ & $\begin{array}{l}\text { Monthl } \\
\text { y }\end{array}$ & $\begin{array}{l}\text { One-of } \\
\text { contac } \\
t\end{array}$ & \\
\hline
\end{tabular}

Department for

Work and

Pensions

Pension Credit

Service/

Pension Credit

Citizen's Advice

Bureau

Council Tax

Reduction Team

Age Cymru/ Age

UK

Age Connects

Carers Centre

Job Centre Plus

Turn 2 Us

Supporting

people

MantellGwyned

d

Other? Please specify names and type of service offered 


\section{(b) Fundraising}

19a. Fundraising: Business- includes any businesses or social enterprises you approach on behalf of a client to find funding to pay for essential maintenance works or other services.

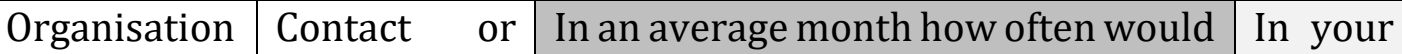
name and collaboration you link with these services (tick opinion logo made (tick if most relevant category) applicable)

\begin{tabular}{|c|c|c|c|c|c|c|}
\hline \multicolumn{6}{|c|}{ applicable) } & \multirow{2}{*}{$\begin{array}{l}\text { good } \\
\text { service } \\
(\mathrm{Y} / \mathrm{N}) ?\end{array}$} \\
\hline & & Daily & Weekly & Monthly & $\begin{array}{l}\text { One-of } \\
\text { contact }\end{array}$ & \\
\hline $\begin{array}{l}\text { British Gas } \\
\text { Energy } \\
\text { Trust }\end{array}$ & & & & & & \\
\hline $\begin{array}{l}\text { Health } \\
\text { through } \\
\text { Warmth/ } \\
\text { Npower }\end{array}$ & & & & & & \\
\hline $\begin{array}{l}\text { North Wales } \\
\text { Energy } \\
\text { Advice } \\
\text { Centre }\end{array}$ & & & & & & \\
\hline Nest/ Nyth & & & & & & \\
\hline $\begin{array}{l}\text { Other? Pl } \\
\text { names and } \\
\text { offered }\end{array}$ & $\begin{array}{l}\text { ase specify } \\
\text { zpe of service }\end{array}$ & & & & & \\
\hline
\end{tabular}




\begin{tabular}{|c|c|c|c|c|c|c|}
\hline \multicolumn{7}{|c|}{$\begin{array}{l}\text { 19b. Fundraising: Public sector-includes any public sector organisations } \\
\text { you approach on behalf of a client to find funding to pay for essential } \\
\text { maintenance works other services. }\end{array}$} \\
\hline \multirow[t]{2}{*}{$\begin{array}{l}\text { Organisation } \\
\text { name and } \\
\text { logo }\end{array}$} & \multirow{2}{*}{$\begin{array}{l}\text { Contact or } \\
\text { collaboration } \\
\text { made (tick if } \\
\text { applicable) }\end{array}$} & \multicolumn{4}{|c|}{$\begin{array}{l}\text { In an average month how often would } \\
\text { you link with these services (tick } \\
\text { most relevant category) }\end{array}$} & \multirow{2}{*}{$\begin{array}{l}\text { In your } \\
\text { opinion } \\
\text { is this a } \\
\text { good } \\
\text { service } \\
(\mathrm{Y} / \mathrm{N}) ?\end{array}$} \\
\hline & & Daily & Weekly & Monthly & $\begin{array}{l}\text { One-of } \\
\text { contact }\end{array}$ & \\
\hline \multicolumn{7}{|l|}{$\begin{array}{l}\text { Health } \\
\text { Board } \\
\text { Council }\end{array}$} \\
\hline \multicolumn{7}{|l|}{ Grants office } \\
\hline \multicolumn{7}{|l|}{$\begin{array}{l}\text { Local } \\
\text { Council/ } \\
\text { Authority }\end{array}$} \\
\hline \multicolumn{7}{|l|}{$\begin{array}{l}\text { Housing } \\
\text { Associations }\end{array}$} \\
\hline $\begin{array}{l}\text { Other? Ple } \\
\text { names and } \\
\text { services offe } \\
\text { you approa } \\
\text { associations } \\
\text { provide their }\end{array}$ & $\begin{array}{l}\text { ase specify } \\
\text { types of } \\
\text { red. Also, if } \\
\text { ch housing } \\
\text { please } \\
\text { names. }\end{array}$ & & & & & \\
\hline
\end{tabular}


19c. Fundraising: Charity- includes registered charitable organisations unrelated to any professional bodies that you approach on behalf of a client to find funding to pay for essential maintenance works or other services

\begin{tabular}{|c|c|c|c|c|c|c|}
\hline \multirow[t]{2}{*}{$\begin{array}{l}\text { Organisation } \\
\text { name and } \\
\text { logo }\end{array}$} & \multirow[t]{2}{*}{$\begin{array}{l}\text { Contact or } \\
\text { collaboratio } \\
\mathrm{n} \text { made (tick } \\
\text { if applicable) }\end{array}$} & \multicolumn{4}{|c|}{$\begin{array}{l}\text { In an average month how often } \\
\text { would you link with these services on } \\
\text { behalf of a client (tick most relevant } \\
\text { category) }\end{array}$} & \multirow{2}{*}{$\begin{array}{l}\text { In your } \\
\text { opinio } \\
\mathrm{n} \text { is this } \\
\mathrm{a} \text { good } \\
\text { service } \\
(\mathrm{Y} / \mathrm{N}) ?\end{array}$} \\
\hline & & $\begin{array}{l}\text { Dail } \\
\mathbf{y}\end{array}$ & $\begin{array}{l}\text { Weekl } \\
\text { y }\end{array}$ & $\begin{array}{l}\text { Monthl } \\
y\end{array}$ & $\begin{array}{l}\text { One- } \\
\text { off } \\
\text { contac } \\
\text { t }\end{array}$ & \\
\hline $\begin{array}{l}\text { National } \\
\text { Benevolence } \\
\text { Charity }\end{array}$ & & & & & & \\
\hline $\begin{array}{l}\text { Silversmiths } \\
\text { Charity }\end{array}$ & & & & & & \\
\hline $\begin{array}{l}\text { Community } \\
\text { Foundation } \\
\text { Fund in } \\
\text { Wales }\end{array}$ & & & & & & \\
\hline $\begin{array}{l}\text { Francis } \\
\text { Winham } \\
\text { Foundation }\end{array}$ & & & & & & \\
\hline $\begin{array}{l}\text { British } \\
\text { Jewellers, } \\
\text { Giftware } \\
\text { Benevolent } \\
\text { Society }\end{array}$ & & & & & & \\
\hline $\begin{array}{l}\text { The Eaton } \\
\text { Fund for } \\
\text { Artists, } \\
\text { Nurses and } \\
\text { Gentlewoma } \\
\text { n }\end{array}$ & & & & & & \\
\hline $\begin{array}{l}\text { The Charity } \\
\text { for Civil } \\
\text { Servants } \\
\text { (Civil Service } \\
\text { Benevolent } \\
\text { Fund) }\end{array}$ & & & & & & \\
\hline $\begin{array}{l}\text { Supporting } \\
\text { Friends }\end{array}$ & & & & & & \\
\hline $\begin{array}{l}\text { Newstraid } \\
\text { Benevolent } \\
\text { fund }\end{array}$ & & & & & & \\
\hline $\begin{array}{l}\text { Lighthouse } \\
\text { Fund }\end{array}$ & & & & & & \\
\hline $\begin{array}{l}\text { Bankworkers } \\
\text { Charity }\end{array}$ & & & & & & \\
\hline
\end{tabular}




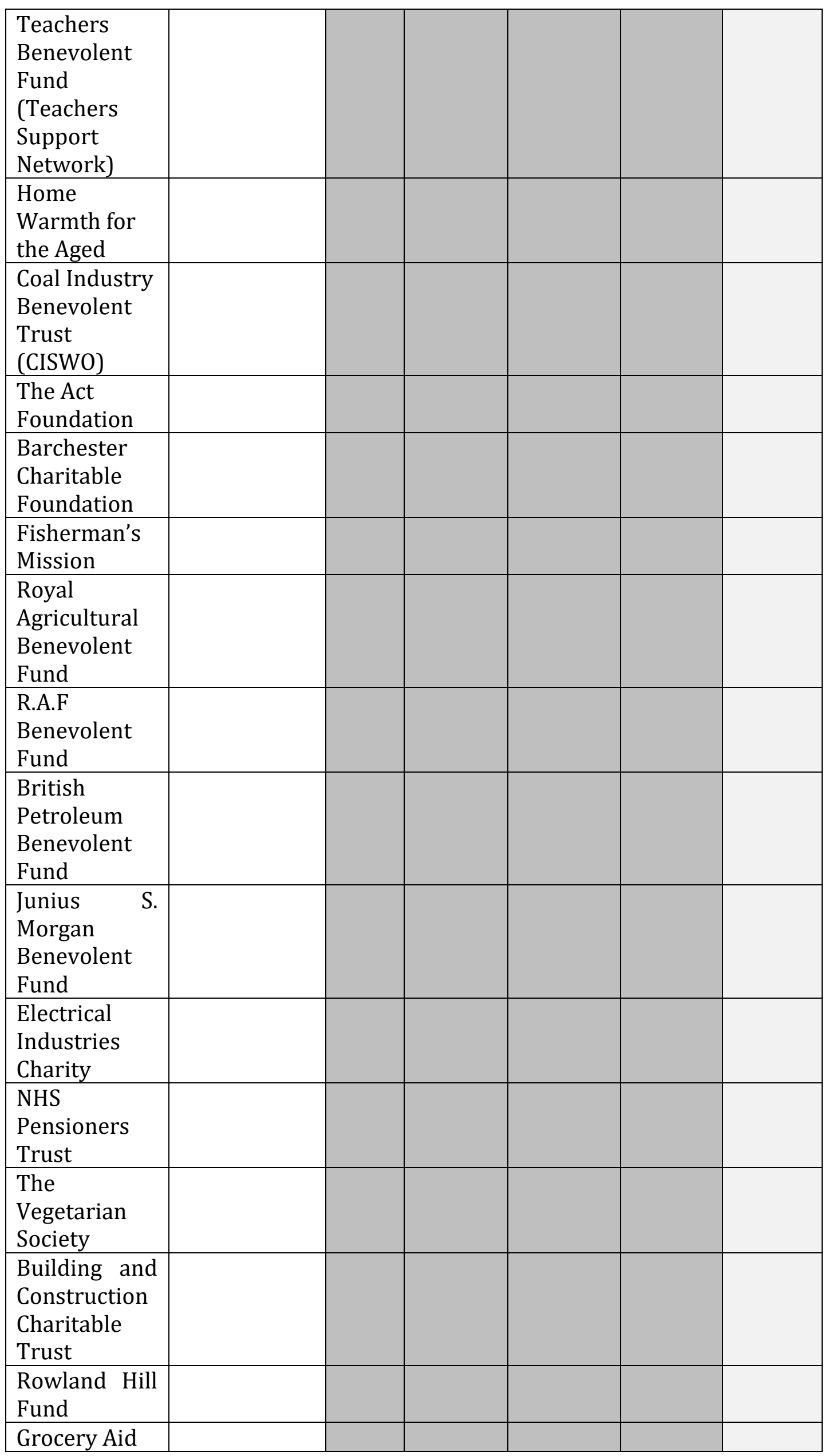




\begin{tabular}{|l|l|l|l|l|l|}
\hline British Gad & & & & & \\
Energy Fund & & & & & \\
\hline $\begin{array}{l}\text { Queens } \\
\text { Nursing }\end{array}$ & & & & & \\
\hline Retail Trust & & & & \\
\hline $\begin{array}{l}\text { Other? Please specify } \\
\text { names and types of } \\
\text { services offered }\end{array}$ & & & & \\
\hline
\end{tabular}


19d. Fundraising: Professional/ occupational-includes organisations that offer benevolent funding based on the occupational background of the client (or their spouse) that you approach on behalf of a client to find funding to pay for essential maintenance works or other services.

\begin{tabular}{l|l|l|l} 
Organisatio & Contact or & In an average month how often & In your
\end{tabular} n name and collaboratio would you link with these services opinion is logo n made

(tick most relevant category)

(tick if applicable)

\begin{tabular}{|c|c|c|c|c|c|c|}
\hline & $\begin{array}{l}\text { (tick if } \\
\text { applicable) }\end{array}$ & & \multirow{2}{*}{$\begin{array}{l}\text { service }(Y / N \\
) ?\end{array}$} \\
\hline & & $\begin{array}{l}\text { Dail } \\
\mathbf{y}\end{array}$ & $\begin{array}{l}\text { Weekl } \\
\mathbf{y}\end{array}$ & $\begin{array}{l}\text { Monthl } \\
\mathbf{y}\end{array}$ & \begin{tabular}{|l}
$\begin{array}{l}\text { One- } \\
\text { off } \\
\text { contac } \\
\text { t }\end{array}$ \\
\end{tabular} & \\
\hline Roya & & & & & & \\
\hline
\end{tabular}

British

Legion

Grocery Aid

Coal Industry

Social

Welfare

Organisatio n (CISWO)

(SSAFA)

Soldier's and Sailor's Family Association

NHS

Pensioners

Fund

Railways

Benevolent

Fund

Royal Air

Force

(RAF)

Cavell Trust

Electrical

and

Electronics

Industries

Benevolent

Association

(EEIBA)

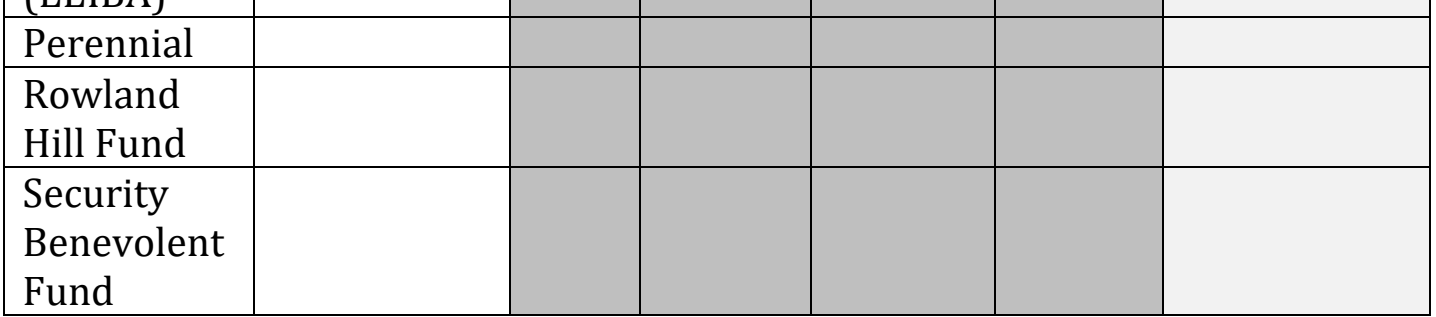


Other? Please specify names and types of services offered 


\section{(c) Making referrals to other services for Personal Support}

20a. Befriending services/ community support: includes organisations you approach or refer clients to when they are in need of emotional support (e.g. bereavement). This can include volunteer services and community organisations

\begin{tabular}{|c|c|c|c|c|c|c|}
\hline \multirow[t]{2}{*}{$\begin{array}{l}\text { Organisation } \\
\text { name and } \\
\text { logo }\end{array}$} & \multirow{2}{*}{$\begin{array}{l}\text { Contact or } \\
\text { collaboration } \\
\text { made (tick if } \\
\text { applicable) }\end{array}$} & \multicolumn{4}{|c|}{$\begin{array}{l}\text { In an average month how often } \\
\text { would you link with these services } \\
\text { (tick most relevant category) }\end{array}$} & \multirow{2}{*}{$\begin{array}{l}\text { In your } \\
\text { opinion } \\
\text { is this a } \\
\text { good } \\
\text { service } \\
(\mathrm{Y} / \mathrm{N}) \text { ? }\end{array}$} \\
\hline & & Daily & Weekly & Monthly & $\begin{array}{l}\text { One-off } \\
\text { contact }\end{array}$ & \\
\hline $\begin{array}{l}\text { Royal } \\
\text { Voluntary } \\
\text { Service } \\
\text { (WRVS) }\end{array}$ & & & & & & \\
\hline $\begin{array}{l}\text { Friends of } \\
\text { the Elderly }\end{array}$ & & & & & & \\
\hline $\begin{array}{l}\text { Ffrindiau/ } \\
\text { Age Cymru }\end{array}$ & & & & & & \\
\hline $\begin{array}{l}\text { Contact the } \\
\text { Elderly }\end{array}$ & & & & & & \\
\hline $\begin{array}{l}\text { Volunteer } \\
\text { Bureau }\end{array}$ & & & & & & \\
\hline $\begin{array}{ll}\text { BT } & \& \\
\text { Silverline } & \\
\end{array}$ & & & & & & \\
\hline $\begin{array}{l}\text { Forget me } \\
\text { Not }\end{array}$ & & & & & & \\
\hline Red Cross & & & & & & \\
\hline $\begin{array}{l}\text { Cruse } \\
\text { Bereavement } \\
\text { Care }\end{array}$ & & & & & & \\
\hline $\begin{array}{l}\text { Community } \\
\text { Groups and } \\
\text { classes: if } \\
\text { applicable, } \\
\text { please } \\
\text { specify } \\
\text { name(s) }\end{array}$ & & & & & & \\
\hline Libraries & & & & & & \\
\hline $\begin{array}{l}\text { Other? Plea } \\
\text { names and } \\
\text { services offe } \\
\text { you appro } \\
\text { community-b } \\
\text { organisations } \\
\text { provide their }\end{array}$ & \begin{tabular}{l} 
Ise \\
\multicolumn{2}{c}{ specify } \\
types of \\
red. Also, if \\
bach local \\
ased \\
names
\end{tabular} & & & & & \\
\hline
\end{tabular}


20b. Re-enablement: includes organisations you approach because they offer re-enablement services and promote independence of clients following a recent illness or stay in hospital.

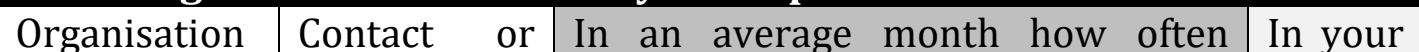
name and collaboration would you link with these services opinion logo $\quad$ made (tick if (tick most relevant category)

\begin{tabular}{|l|l|l|l|l|l|l|} 
& applicable & \multicolumn{3}{|l|}{} & $\begin{array}{l}\text { good } \\
\text { service } \\
\text { (Y/N)? }\end{array}$ \\
\hline $\begin{array}{l}\text { Disability Can } \\
\text { Do }\end{array}$ & Daily & Weekly & Monthly & $\begin{array}{l}\text { One- } \\
\text { off } \\
\text { contact }\end{array}$ & & \\
\hline Sight Cym & & & & & & \\
\hline
\end{tabular}

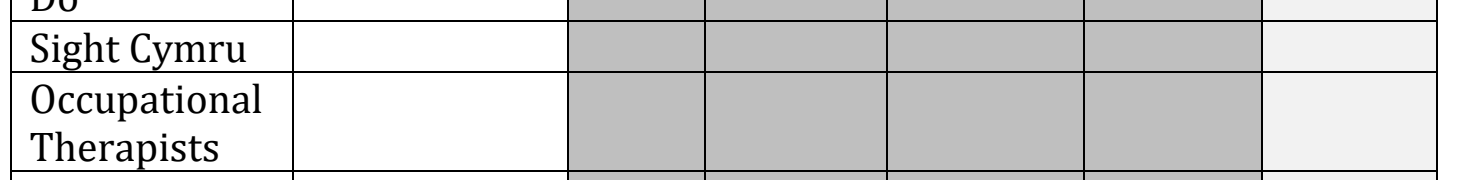

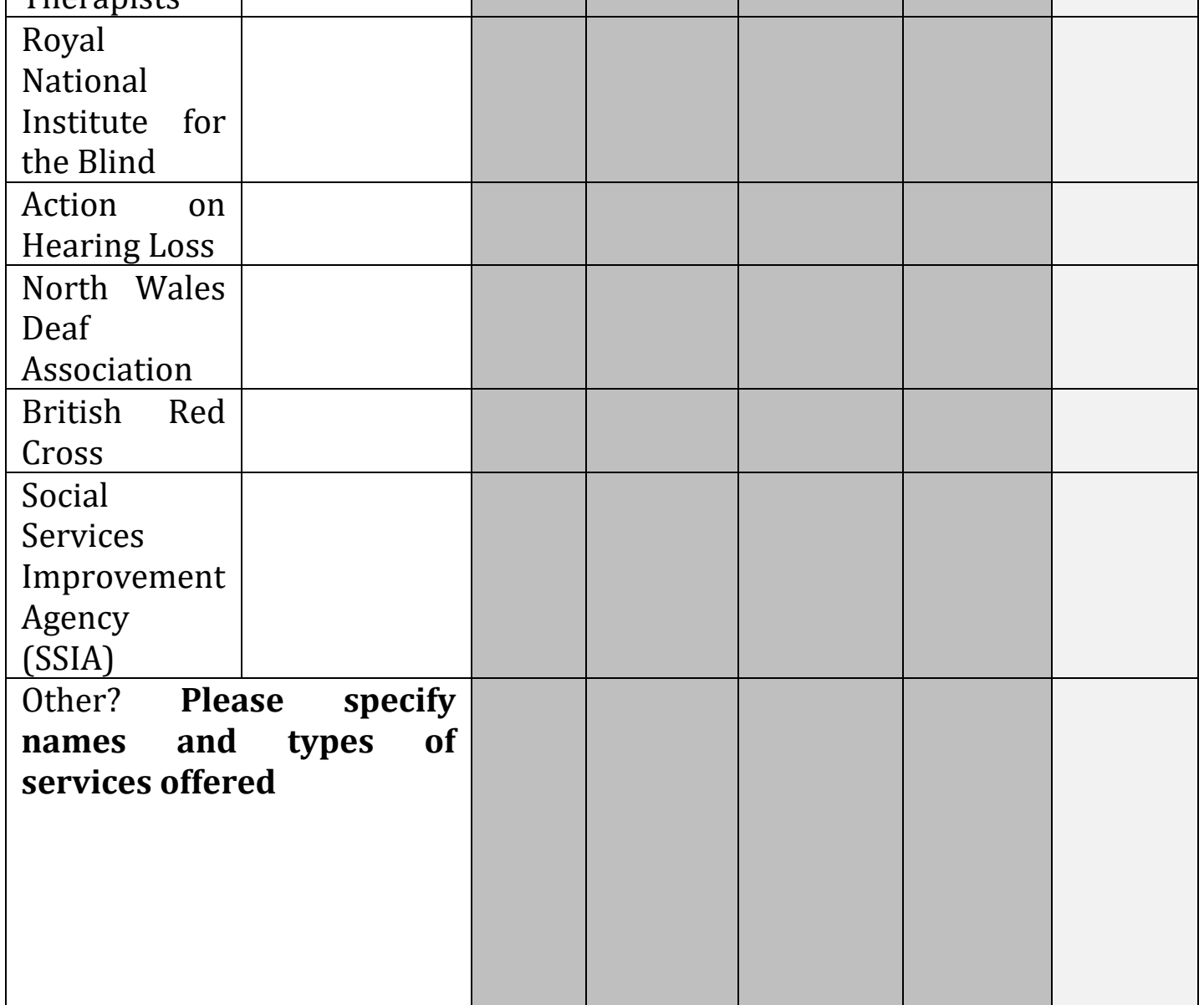


20c. Health: includes organisations you approach because they offer support to clients who have health, mobility, sensory or cognitive impairments or issues.

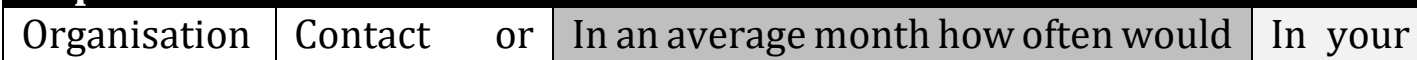
name and collaboration you link with these services (tick opinion logo made (tick if most relevant category)

\begin{tabular}{|c|c|c|c|c|c|c|}
\hline - & applicable) & & & & & good \\
\hline & & Daily & Weekly & Monthly & $\begin{array}{l}\text { One-off } \\
\text { contact }\end{array}$ & service \\
\hline
\end{tabular}

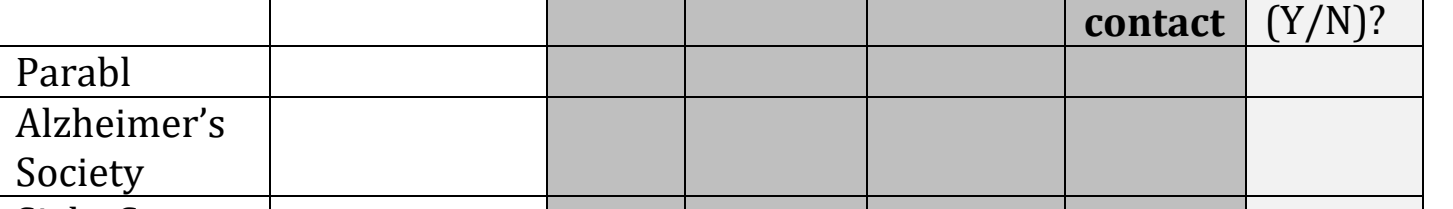

\begin{tabular}{|l|l|l|l|l|l|}
\hline Sight Cymru & & & & & \\
\hline Heart & & & & & \\
\hline
\end{tabular}

Foundation

Occupational therapists

Royal

National

Institute for the Blind

Action on

Hearing Loss

Mental

Health Team

Crossroads

Vest

Community

Transport

Other? Please specify

names and types of

services offer: 
20d. Personal Care: includes organisations you approach or refer a client to for support with their personal care (this includes things such as washing and dressing).

\begin{tabular}{|l|l|l|l|l|l|l|}
$\begin{array}{l}\text { Organisation } \\
\text { name } \\
\text { Logo }\end{array}$ & $\begin{array}{l}\text { Contact or } \\
\text { collaboration } \\
\text { made (tick if } \\
\text { applicable) }\end{array}$ & \multicolumn{2}{|l|}{$\begin{array}{l}\text { In an average month how often would } \\
\text { you link with these services (tick } \\
\text { most relevant category) }\end{array}$} & $\begin{array}{l}\text { In your } \\
\text { opinion } \\
\text { is this a } \\
\text { good } \\
\text { service } \\
\text { (Y/N)? }\end{array}$ \\
\hline $\begin{array}{l}\text { Local } \\
\text { Authority/ } \\
\text { Local } \\
\text { Council }\end{array}$ & Daily & Weekly & Monthly & $\begin{array}{l}\text { One-off } \\
\text { contact }\end{array}$ & & \\
\hline $\begin{array}{l}\text { Crossroads } \\
\text { Please specify } \\
\text { types of }\end{array}$ & & & & & \\
\hline $\begin{array}{l}\text { Other? } \\
\text { names } \\
\text { services offered }\end{array}$ & & & & & \\
\hline
\end{tabular}

20e. Domestic Help: includes organisations you approach or refer clients into for minor domestic support e.g. dog walkers, cleaners and transport.

\begin{tabular}{|l|l|l|l|l|l|l|}
\hline $\begin{array}{l}\text { Organisation } \\
\text { name and } \\
\text { logo }\end{array}$ & $\begin{array}{l}\text { Contact or } \\
\text { collaboration } \\
\text { made (tick if } \\
\text { applicable) }\end{array}$ & \multicolumn{3}{|l|}{$\begin{array}{l}\text { In an average month how often would } \\
\text { you link with these services on behalf } \\
\text { of client (tick most relevant } \\
\text { category) }\end{array}$} & $\begin{array}{l}\text { In your } \\
\text { opinion } \\
\text { is this a } \\
\text { good } \\
\text { service } \\
\text { (Y/N)? }\end{array}$ \\
\hline $\begin{array}{l}\text { Wiltshire } \\
\text { Farm Foods }\end{array}$ & Daily & Weekly & Monthly & $\begin{array}{l}\text { One-off } \\
\text { contact }\end{array}$ & & \\
\hline $\begin{array}{l}\text { Cinnamon } \\
\text { Trust }\end{array}$ & & & & & & \\
\hline $\begin{array}{l}\text { Vest } \\
\text { community } \\
\text { Transport }\end{array}$ & & & & & \\
\hline $\begin{array}{l}\text { Mail and } \\
\text { Telephone } \\
\text { Preference } \\
\text { Service }\end{array}$ & & & & & \\
\hline Dial Ride & & & & & \\
\hline $\begin{array}{l}\text { Other? Please specify } \\
\text { names and types of } \\
\text { services offered }\end{array}$ & & & & & \\
\hline
\end{tabular}


20f. Digital inclusion: includes organisations you approach to help clients become more confident in using digital technologies in their everyday lives, including for health prevention or as part of a care plan (i.e. falls prevention).

\begin{tabular}{|l|l|l|l|l|l|l|}
\hline $\begin{array}{l}\text { Organisation } \\
\text { name and } \\
\text { logo }\end{array}$ & $\begin{array}{l}\text { Contact or } \\
\text { collaboration } \\
\text { made (tick if } \\
\text { applicable) }\end{array}$ & \multicolumn{2}{|l|}{$\begin{array}{l}\text { In an average month how often would } \\
\text { you link with these services (tick } \\
\text { most relevant category) }\end{array}$} & $\begin{array}{l}\text { In your } \\
\text { opinion } \\
\text { is this a } \\
\text { good } \\
\text { service } \\
\text { (Y/N)? }\end{array}$ \\
\hline Careline & Daily & Weekly & Monthly & $\begin{array}{l}\text { One-off } \\
\text { contact }\end{array}$ & & \\
\hline Telecare & & & & & & \\
\hline $\begin{array}{l}\text { RNIB Online } \\
\text { Today } \\
\text { Project }\end{array}$ & & & & & \\
\hline Libraries & & & & & \\
\hline $\begin{array}{l}\text { Other? Please specify } \\
\text { names and types of } \\
\text { services offered }\end{array}$ & & & & & \\
\hline
\end{tabular}




\section{(d) Healthy Homes Check}

21a. Fuel Poverty: includes organisations you approach or refer clients onto to help prevent or stop fuel poverty.

\begin{tabular}{|c|c|c|c|c|c|c|}
\hline \multirow[t]{2}{*}{$\begin{array}{l}\text { Organisation } \\
\text { name } \\
\text { Logo }\end{array}$} & \multirow{2}{*}{$\begin{array}{l}\text { Contact or } \\
\text { collaboration } \\
\text { made (tick if } \\
\text { applicable) }\end{array}$} & \multicolumn{4}{|c|}{$\begin{array}{l}\text { In an average month how often would } \\
\text { you link with these services (tick } \\
\text { most relevant category) }\end{array}$} & \multirow{2}{*}{$\begin{array}{l}\text { In your } \\
\text { opinion } \\
\text { is this a } \\
\text { good } \\
\text { service } \\
(\mathrm{Y} / \mathrm{N}) ?\end{array}$} \\
\hline & & Daily & Weekly & Monthly & $\begin{array}{l}\text { One-off } \\
\text { contact }\end{array}$ & \\
\hline $\begin{array}{l}\text { Npower/ } \\
\text { Health } \\
\text { through } \\
\text { Warmth }\end{array}$ & & & & & & \\
\hline SWALEC & & & & & & \\
\hline $\begin{array}{l}\text { NEST/ ECO } \\
\text { Scheme }\end{array}$ & & & & & & \\
\hline Heatforce & & & & & & \\
\hline $\begin{array}{l}\text { Energy } \\
\text { Savings } \\
\text { Trust/NEST }\end{array}$ & & & & & & \\
\hline $\begin{array}{l}\text { British Gas } \\
\text { Energy Trust }\end{array}$ & & & & & & \\
\hline $\begin{array}{l}\text { Other? Ple } \\
\text { names and } \\
\text { services offe }\end{array}$ & $\begin{array}{l}\text { specify } \\
\text { types of } \\
\text { ed }\end{array}$ & & & & & \\
\hline
\end{tabular}

\section{1b. Fuel Safety: includes organisations that you approach to ensure} clients' homes are safe in terms of gas and fuel safety issues

\begin{tabular}{|l|l|l|l|l|l|l|}
\hline $\begin{array}{l}\text { Organisation } \\
\text { name } \\
\text { Logo }\end{array}$ & $\begin{array}{l}\text { Contact or } \\
\text { collaboration } \\
\text { made (tick if } \\
\text { applicable) }\end{array}$ & \multicolumn{2}{|l|}{$\begin{array}{l}\text { In an average month how often } \\
\text { (tick most relevant category) }\end{array}$} & $\begin{array}{l}\text { In your } \\
\text { opinion } \\
\text { is this a } \\
\text { good } \\
\text { service } \\
\text { (Y/N)? }\end{array}$ \\
\hline Fire Service & Daily & Weekly & Monthly & $\begin{array}{l}\text { One- } \\
\text { off } \\
\text { contact }\end{array}$ & & \\
\hline $\begin{array}{l}\text { NEST/ ECO } \\
\text { Scheme }\end{array}$ & & & & & \\
\hline $\begin{array}{l}\text { Trading } \\
\text { Standards/ } \\
\text { POVA Team }\end{array}$ & & & & & \\
\hline $\begin{array}{l}\text { Other? Please specify } \\
\text { names and types of } \\
\text { services offered }\end{array}$ & & & & \\
\hline
\end{tabular}


21c. Slips, trips and falls prevention: includes organisations that you approach on behalf of the client to prevent slips, tips and falls in the home.

\begin{tabular}{|l|l|l|l|l|l|}
\hline $\begin{array}{l}\text { Organisation } \\
\text { name and } \\
\text { logo }\end{array}$ & $\begin{array}{l}\text { Contact or } \\
\text { collaboration } \\
\text { made (tick if } \\
\text { applicable) }\end{array}$ & \multicolumn{2}{|l|}{$\begin{array}{l}\text { In an average month how often would } \\
\text { you link with these services (tick }\end{array}$} & $\begin{array}{l}\text { In your } \\
\text { opinion } \\
\text { is this a } \\
\text { good } \\
\text { service } \\
\text { (Y/N)? }\end{array}$ \\
\hline $\begin{array}{l}\text { Community } \\
\text { Safety Team }\end{array}$ & & Daily & Weekly & Monthly & $\begin{array}{l}\text { One-off } \\
\text { contact }\end{array}$ \\
\hline Telecare & & & & & \\
\hline Careline & & & & & \\
\hline $\begin{array}{l}\text { British Red } \\
\text { Cross }\end{array}$ & & & & & \\
\hline Falls Team & & & & & \\
\hline $\begin{array}{l}\text { Social } \\
\text { Workers } \\
\text { (Local } \\
\text { Authority) }\end{array}$ & & & & & \\
\hline $\begin{array}{l}\text { Social } \\
\text { workers } \\
\text { (Hospital) }\end{array}$ & & & & & \\
\hline $\begin{array}{l}\text { Doctors/ G. } \\
\text { Ps }\end{array}$ & & & & & \\
\hline $\begin{array}{l}\text { Other? please specify } \\
\text { names and } \\
\text { services offered } \\
\text { types }\end{array}$ & & & & & \\
\hline
\end{tabular}

21d. Security of the Home: includes organisations you contact to because they aim to ensure a client's home is safe and secure against criminal acts (this includes minor housing adaptations and community security schemes)

\begin{tabular}{|l|l|l|l|l|l|l|}
\hline $\begin{array}{l}\text { Organisation } \\
\text { name and } \\
\text { logo }\end{array}$ & $\begin{array}{l}\text { Contact or } \\
\text { collaboration } \\
\text { made (tick if } \\
\text { applicable) }\end{array}$ & \multicolumn{2}{|l|}{$\begin{array}{l}\text { In an average month how often } \\
\text { would you link (tick most relevant } \\
\text { category) }\end{array}$} & $\begin{array}{l}\text { In your } \\
\text { opinion } \\
\text { is this a } \\
\text { good } \\
\text { service } \\
\text { (Y/N)? }\end{array}$ \\
\hline $\begin{array}{l}\text { Trading } \\
\text { Standards/ } \\
\text { POVA Team }\end{array}$ & Daily & Weekly & Monthly & $\begin{array}{l}\text { One-off } \\
\text { contact }\end{array}$ & & \\
\hline Bobby Van & & & & & \\
\hline $\begin{array}{l}\text { Other? please specify } \\
\text { names and types of } \\
\text { services offered }\end{array}$ & & & & & \\
\hline
\end{tabular}

(e) Home maintenance and adaptations 


\section{Includes any organisations that you approach or refer clients to when}

their property (including the external surroundings) needs building works and adaptations (both minor and extensive).

\begin{tabular}{|c|c|c|c|c|c|c|}
\hline \multirow[t]{2}{*}{$\begin{array}{l}\text { Organisation } \\
\text { name and } \\
\text { logo }\end{array}$} & \multirow{2}{*}{$\begin{array}{l}\text { Contact or } \\
\text { collaboration } \\
\text { made (tick if } \\
\text { applicable) }\end{array}$} & \multicolumn{4}{|c|}{$\begin{array}{l}\text { In an average month how often would } \\
\text { you link with these services (tick } \\
\text { most relevant category) }\end{array}$} & \multirow{2}{*}{$\begin{array}{l}\text { In your } \\
\text { opinion } \\
\text { is this a } \\
\text { good } \\
\text { service } \\
(\mathrm{Y} / \mathrm{N}) ?\end{array}$} \\
\hline & & Daily & Weekly & Monthly & $\begin{array}{l}\text { One-off } \\
\text { contact }\end{array}$ & \\
\hline $\begin{array}{l}\text { Local } \\
\text { contactor(s): } \\
\text { if applicable } \\
\text { please } \\
\text { specify } \\
\text { name(s) }\end{array}$ & & & & & & \\
\hline $\begin{array}{l}\text { Housing } \\
\text { Department }\end{array}$ & & & & & & \\
\hline Melin & & & & & & \\
\hline Derwen & & & & & & \\
\hline Age UK & & & & & & \\
\hline Poppy Call & & & & & & \\
\hline $\begin{array}{l}\text { Carers } \\
\text { centre }\end{array}$ & & & & & & \\
\hline $\begin{array}{l}\text { Gerddi } \\
\text { Gwyrrd }\end{array}$ & & & & & & \\
\hline $\begin{array}{l}\text { Care \& } \quad \& \\
\text { Repair- } \\
\text { social } \\
\text { enterprise }\end{array}$ & & & & & & \\
\hline $\begin{array}{l}\text { Grounds for } \\
\text { Action }\end{array}$ & & & & & & \\
\hline Discovery & & & & & & \\
\hline $\begin{array}{l}\text { Local } \\
\text { college(s). If } \\
\text { applicable, } \\
\text { please } \\
\text { specify } \\
\text { name(s) }\end{array}$ & & & & & & \\
\hline $\begin{array}{l}\text { Other? Ple } \\
\text { names anc } \\
\text { services offe }\end{array}$ & $\begin{array}{l}\text { sse specify } \\
\text { types of } \\
\text { ed }\end{array}$ & & & & & \\
\hline
\end{tabular}




\section{Section E- Issues on the ground}

In this last section, we would like to seek your point of view about the things that can sometimes make it challenging or difficult for you to carry out your work as a caseworker on the ground, and the work that you may undertake in "going that extra mile" to support clients.

23. From the list below, please indicate the factors that correspond to the sorts of difficulties you may have experienced in carrying out your work as a caseworker.

a) Organisations and

services are reluctant to

b) Difficulties

work together (e.g. accessing clients

c) ICT problems (e.g.

competition for clients,

who live in remote poor internet

funding resources etc.) areas

d) Client reluctant to accept help

e) Client's objections to giving personal

f) Client is unhappy access) details e.g. about changing

financial their home environment information

g) Couples disagree between themselves

h) Client's family objects to C\& R

i) Limited funds about the support interventions available to help support needed client's needs

j) Changing to benefits eligibility criteria

k) Language barriers

l) Organisational restructuring

m) Time needed to

complete administrative tasks

Please tell us about any other challenges or difficulties we have missed by writing down your thought in as much detail as possible in the box below: 
24. From your point of view as a caseworker, do you think there are things that could be done to improve your working environment or role within Care \& Repair? (e.g. more training). Please write down your thoughts in as much detail as possible in the box below:

25. Like many jobs, there may be several things that caseworkers do to "go the extra mile" in helping to support their clients, which are not reflected in their job description, but which contribute significantly to helping improve outcomes for clients. Please write down your thoughts about this in as much detail as possible using the box below:

26. To end the questionnaire, I would like to know if there is anything about your role as a caseworker that I have missed out which is important to you. I would be really interested to read what you have to say. (Please write down your thoughts in the box below and on the next page if necessary).

This is the end of the questionnaire. 
Thank you for taking part!

I really appreciate that you have had to give up your time to take part in this study. Your views and experiences are very important and will be used to inform the outcomes of this study.

Contact for follow-up interviews and focus group participation

Due to the complexity of the research, I would like to seek your permission to contact you again either to ensure all data has been correctly recorded or to invite you to take part in a focus group.

The follow-up focus group will discuss some of the issues or themes that have arisen in the questionnaire. Please be assured that all personal information that is collected about you will be kept confidential. I will not include your name or any other information that might identify you personally. But your anonymised answers may be used as points of discussion in this focus group.

Please note that a follow-up interview may not be required but if you would be happy to be contacted for a follow up interview or be invited to participate in a focus group, could you please sign your name below:

I am happy to be contacted for follow-up interviews if deemed necessary by the researcher or be approached to participate in a focus group with other caseworkers

Signed:

Date:

Once I have received your completed questionnaire back, I will send you a debriefing sheet. This debriefing sheet will give you information on what will happen next and for the contact information of the researcher.

Thank you once again!

Joanna L. Wolton 


\section{Appendix Item 4: Chief Officer Interview schedule}

Introduction:

Thank you for agreeing to take part and taking time out of your day to come and speak to me. I am speaking to you today because I am interested in understanding more about the case worker role and the broader context in which they care working.

The reason I am speaking to you is because you have knowledge about the local and national contexts in which the case workers and your agency(ies) are based. Given the very diverse nature of Wales' counties and their older inhabitants, it is important to have agency managers' perspectives about how local contexts shape the types of services available to their older clients at the local level (e.g. local level partnerships between organizations; funding; cultural and linguistic context etc.)

The information you give me today will help me inform the topics covered and the questions used in the questionnaire that will be sent out to the case workers in the New Year. Your answers will also help with the analysis of the questionnaire results.

Is there anything you would like to ask me or clarify before we begin?

1. Which Care \& Repair Agency(ies) are you responsible for managing?

Prompts: So how many staff do you manage overall? Number of CWs?

2. Can you give me a rough idea of your current client base?

Prompts: Age; Gender; Socio-economic background

3. As you can probably tell from my accent, I am not from Wales, could you tell me a bit about the local area in which your agency(ies) are based?

4. Imagine I am a new recruit to Care \& Repair, from your perspective as a manager how would you define the $\mathrm{CW}$ role to me?

Prompt: What is it exactly that makes them so vital to the organization?

5. What would you say are the main tasks of the $\mathrm{CW}$ role?

Prompt: What sorts of activities do they undertake as part of their role?

6. Could you tell me roughly how many visits case workers make in an average month?

Prompts: How many are first/ initial visits to clients? How many are follow-up visits roughly?

7. One thing that I noticed when visiting some agencies is that the working relationship between CWs and TOs are very different depending on the agency. From my observation I noticed that there were some agencies in which the two roles worked closely together, for instance they would go on joint visits. However, there were others where CWs and TOs would communicate with each other, but they would work more independently from one another. Have I understood this correctly?

Prompt: How do the two roles interact in the agency(ies) you manage?

8. How are the clients divided between case workers, i.e. do the case workers work in patches/ assigned to a particular area of the county?

9. Who would you say are the main organizations or partners (these can be both non-governmental and governmental) who you link with regularly? 
Prompts: Do you tend to work closely with the local authorities? And how about the health boards (ask name...)?

10. At the moment there seems to be a real emphasis on cross-sector working. I was wondering what your experiences or perspective on this was... does this work in reality?

Prompts: Are there factors that can make this more difficult? Are some organizations easier to work with others? Why?

11. Have you seen a change in the demand for specific types of services? Prompts: Is there a particular service that has become more popular recently? Is there a change in what the client wants and needs now compared to a few years ago? In your opinion, why do you think this is?

12. From your perspective as a manager/Chief Officer what would you say are the main challenges and difficulties faced by the case workers on the ground?

13. Could you tell me if there are any wider reaching issues that influence the functioning of the agency and hence influence the types of activity/ work that CWs are able to carry out? For example, changes of LA funding, or mergers of different services?

Prompts: I assume you are talking about the recent merger of Care and Repair Agencies? Are there any other factors that have an impact on the work that case workers are able to undertake?

14. Are there any services that you don't currently offer which have been requested by some older people?

Prompt: Is there a particular service that you think will become more in demand in the future?

15. Based on what you have told me so far, is there anything else you want to tell me that will help me better understand the $\mathrm{CW}$ role and the broader contexts in which they are working? 


\section{Appendix Item 5: Client Interview schedule}

1. How did you first come to hear about Care \& Repair and what they do?

a. What did you think it was that they do?

2. Why did you link with Care \& Repair/ what was the point of their visit?

3. How did you get in contact with them?

a. Did they approach you?

b. Meet them at an event?

c. Heard about them from a friend?

d. Looked up online?

e. Referred by other agency e.g. GP or DWP or Age Cymru (etc.)?

4. Can you take me through what happened after you contacted them/ they contacted you?

a. Did you speak with them on the phone?

b. Did they arrange a visit?

c. How did you have to wait for them to come and see you?

5. What happened when they came to see you? / What did you talk about?

6. Did they only visit you once/ at all?

7. What happened after they visited you? / What did they do?

a. Did they visit you again?

b. Speak with them on the phone?

c. Contractors came round?

d. Put you in touch with other agencies?

8. Do you think your life has changed at all since you contacted/ were contacted by Care \& Repair?

a. How/ on what way/s?

9. What was your overall experience of Care \& Repair?

a. What did you think of them?

b. Is there anything that you know of that they don't do/ offer that would be useful?

c. Would you recommend them to others/ friends/ family?

10. What would you have done if Care \& repair were not there?

11. Is there anything else you would like to tell me about your experience with Care \& Repair, to help me better understand your point of view? 


\section{Appendix Item 6: Technical Officer Interview schedule}

Thank you for agreeing to take part in this research project and taking time out of your work to attend this networking meeting.

The reason I am here speaking to you all as Technical Officers is that as I'm sure you are aware, I am interested in understanding more about the caseworker role and the broader context in which they are working.

In your role as a Technical Officer, you have a good knowledge of your role in addition to the demands and availability of services in your local area. Therefore, after speaking with you all today I am hoping to understand a bit more about the work that is carried out by the Technical service and how this service links into, and compliments the casework service

The information you give me today will help me inform the topics covered and the questions used in the questionnaire that will be sent out to the caseworkers in the over the month of February. Your answers will also help with the analysis of the questionnaire results.

So, if you are happy to participate would you fill in the consent form for me? Just to let you all know participation is voluntary and you can drop out at any time. It will be audio recorded. If a question is unclear, please let me know and hopefully we can generate some interesting conversation. Are there any questions before we begin?

1. So, I suppose first it may be useful for me if you could all introduce yourselves, which agency you work for, and your job title as I know there's a bit of variation there.

2. Thank you for that. So, to start off I would like to ask you what is the role of a Technical Officer? That is, how would you define the role of a technical officer?

- And how does this relate to the ethos of Care \& Repair?

3. What sorts of activities do you do in your role as a technical officer? 
- Is that the same across the board or does anyone else have anything else to add?

4. In terms of the clients do you tend to work more with those who pay for private works or are they funded by the Local Authority?

- Or does it tend to be a mixture of the two?

- If it is a mixture what would you say is the ratio of private works to funded works?

5. I was wondering if you would be able to tell me how many visits you would carry out in an average month?

- Are they initial or follow-ups?

6. Have the needs of the clients changed recently? So, do you find yourselves overseeing particular works more frequently?

- For instance, is a particular adaptation being prescribed more to clients?

- Are clients asking for particular adaptations more often?

7. How would you define your relationship to the caseworker role/ service?

- Do you have a lot of involvement with case workers directly?

- For instance, do you ever do out on joint visits or do they refer on to you?

- Is your role more independent/ do you tend to primarily work independently from case workers?

- Who is the first point of contact for the client within your agency?

8. From your perspective as an individual who works as part of a frontline service, what would you say are the main challenges do you face in your role?

- $\quad$ Funding

- Increasing demand for services

- Lack of services

- Complexity of cases 
9. Would you say that caseworkers face a similar set of challenges?

10. Based on what we have been discussing so far is there anything else you would like to tell me that will help me better understand both the casework and technical service and the broader contexts in which you are all working? 
Appendix Item 7: Caseworker interview schedule for focus groups

1. Would anyone like to start us off with the first question with what's come out of your discussions?

2. What does the notion of support mean to you? Think about support between caseworkers; support from Care \& Repair as an organisation; support for clients).

3. What does going the extra mile mean to you?

4. Is there more uncertainty in your job role now?

5. Do you feel as though you have enough hours in the day? / Do you ever feel pressed for time in your job role? 
Appendix Item 8- Client information pack

College of Human and Health Sciences

Coleg y Gwyddorau Dynol ac lechyd

Swansea University

Prifysgol Abertawe

I am pleased to invite you to take part in my research study exploring the role that Care \& Repair Cymru Case workers have in helping older people retain their independence so that they can live in their own homes and communities. Before you decide if you would like to participate, it is important for you to understand why the research is being done and what participation will involve.

Please take some time to read the following information carefully, and if anything remains unclear or if you would like some further information please feel free to get in contact using the information listed at the end of this form.

You might want to discuss this invitation with your relatives, friends and other professionals. I would like to stress that you do not have to accept this invitation and should only agree to take part if you want to.

Thank you for taking the time to read this.

\section{This form is available in large print. If you require large font, please contact Joanna L. Wolton, Centre for Innovative Ageing,}

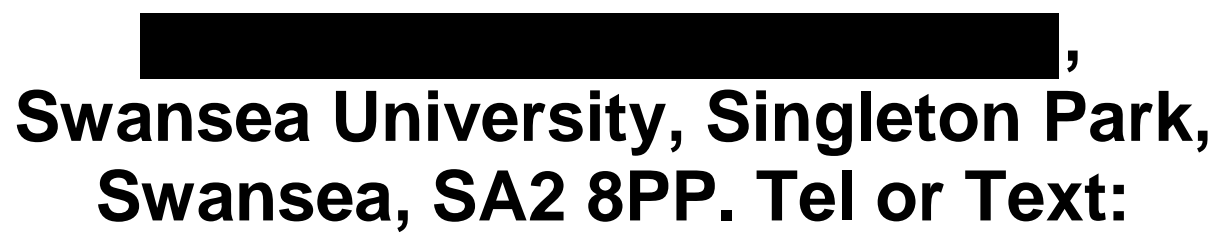

Email: 


\section{What is the purpose of the study?}

Helping older people to remain in their own homes and communities for as long as possible is an issue that is currently high on the agenda of the Welsh Government, and funding has been made available to meet this goal. Care \& Repair Cymru are one of the organisations working with the Welsh Government to achieve this.

The aim of this study is to explore the role that Care \& Repair Cymru play in helping older people retain their independence and live in their own homes and communities for as long as possible. In particular the research will be focusing on the role that Care \& Repair Cymru caseworkers undertake.

However, this research is also interested in the views and experiences of the older clients who have approached Care \& Repair agencies. Therefore, part of this study will look at how the clients themselves believe that their lives have changed as a result of the intervention of their local Care \& Repair agency. My research will provide evidence about the contributions that the Care \& Repair agencies across Wales make in helping older people to remain independent, and about the need for policies and finding to help support these services into the future. This is important because for Care \& Repair Cymru services to continue the work they undertake, and the outcomes of their interventions need to be highlighted and explored.

\section{Why have we been chosen to take part?}

You have been approached because you are aged over 65, living in your own home in Wales, have been a client of your local Care \& Repair agency and have made use of their services.

\section{Do I have to take part?}

You have no obligation to take part in this research. Participation is entirely voluntary, and you are free to withdraw at any time, without explanation and without being disadvantaged in anyway.

\section{What will happen if I decide to take part?}

If you feel satisfied that you have been given enough information about the study and would like to take part, you will be asked to sign the attached consent form and send it to the address listed on page 1 within a week of receiving this information pack.

\section{What will happen if I take part?}

Once I receive the completed consent form, I will contact you via telephone to arrange a time and date for an interview to take place that is convenient for both you and the researcher. This will take place in your home (if you feel comfortable), however, if this is not suitable then we can arrange for an alternative venue for the interview to take place.

During the interview, I will ask you a series of questions about your experiences of your local Care \& Repair agency and how you think their interventions or services have affected your life (if at all). This will last approximately one hour and will be audio-recorded.

\section{Why is the interview tape-recorded?}

The interview will be recorded so that I do not miss anything you say. The recordings will be written up and anonymised so that there are no identifying names or places in the record. The recording will be securely stored by Swansea University and encrypted. 


\section{Expenses and/or payment?}

There will be no expenses or payment for your involvement in this project.

\section{Are there any risks in taking part in the research?}

There are no risks involved in taking part, but it is possible you may find some of the questions upsetting. If you do not want to answer any of the questions you do not have to, please just tell me that you would prefer to not answer, and I will move onto the next question.

\section{Are there any benefits in taking part in the research project?}

There are no direct benefits for the individuals who participate in the study. I hope that the results will aid the local and national government to improve services and outcomes that will enable older individuals to live independently in the community.

\section{Will my participation be kept confidential?}

All information that is collected about you for the study will be kept confidential. I will not include your name or any other information that might identify you in the written record of the interview. The only circumstances in which I would let anyone know you are taking part would be if I felt you may be at risk of harming yourself or someone else.

\section{Will my taking part be covered by an insurance scheme?}

Those who participate in this ethically approved study have insurance cover provided by Swansea University.

\section{What will happen to the results of the study?}

Anonymised data will be stored on a secure, password protected computer for me to analyse during the course of the study. I will not use your name or any other identifying information in the research thesis.

The anonymised data will be stored on a secure, password protected computer. This data will be kept by the researcher beyond the end of the duration of the research project (for approximately five years) and will be used by the researcher and members of the supervisory team to present findings at conferences, on our website and in journal articles. Therefore, the findings of the research will be publicly available.

If you are interested in receiving a copy of the results in a form of executive summary or as a full thesis then the researcher can send you a copy of the findings upon the completion of the study (this is anticipated to be around September 2017).

\section{What will happen if I decide I no longer want to take part?}

Participation is entirely voluntary, and you can withdraw from the study at any time without explanation. Results that are already written up at the time of withdrawal may still be used, only if you are happy for this to occur. Otherwise, you may request that they are destroyed and that no further use is made of them. Your decision will not count against you or disadvantage you in any way.

\section{Who can I contact if I have further questions?}

You can call, email or write to me with any concerns or questions using my contact details below. Please ask questions as often as you want. I am available to take telephone calls Monday-Friday between 9.30am and 3.30pm. You can also contact my supervisor, Dr Sarah Hillcoat-Nallétamby and a representative to Care \& Repair. 


\begin{tabular}{|c|c|}
\hline $\begin{array}{l}\text { Joanna Wolton (PhD } \\
\text { Researcher) } \\
\text { Centre for Innovative Ageing }\end{array}$ & $\begin{array}{l}\text { Supervisor: Dr Sarah Hillcoat- } \\
\text { Nallétamby } \\
\text { Associate Professor } \\
\text { College of Human and Health } \\
\text { Sciences, } \\
\text { Centre for Innovative Ageing } \\
\text { Swansea University } \\
\text { Swansea } \\
\text { SA2 8PP } \\
\text { Tel: } \\
\text { Email: }\end{array}$ \\
\hline
\end{tabular}


Name:

\section{Consent Form}

Address:

Home telephone number:

Mobile:

Email:

1. I confirm that I have read and understood the participant information sheet attached to this form and agree to take part in the above research.

(Please tick and initial).

2. I understand what my role will be in this research project, and all my questions have been answered to my satisfaction by the researcher.

(Please tick and initial).

3. I understand that my right to withdraw from the research at any time, for any reason and without prejudice.

\section{ㅁlease tick and initial).}

4. I have been informed that any information I provide will be anonymised and that the confidentiality of the information will be safeguarded.

(Please tick and initial).

5. I consent to the interview being audio recorded for research purposes.

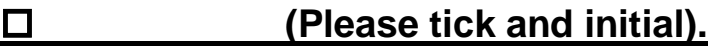

6. I understand that I am free to ask any questions at any time before and during the study

\section{믈 (Please tick and initial).}

7. I have been provided with a copy of this form and the participant information sheet.

\section{ㅁlease tick and initial).}

Data protection: I agree to the University processing personal data that I have supplied. I agree to the processing of such data for any purposes connected with the Research Project as outlined to me.

\section{Name of Participant}

(Print)

Signed

Date

\section{Witnessed by:}

(Print)

Signed

Date 
This form is available in large print. If you require large font, please contact Joanna L. Wolton, Centre for Innovative Ageing,

Swansea University, Singleton Park, Swansea, SA2 8PP. Tel or Text:

Email: 
Appendix Item 9- Figure showing part of the Microsoft Excel SNA matrix

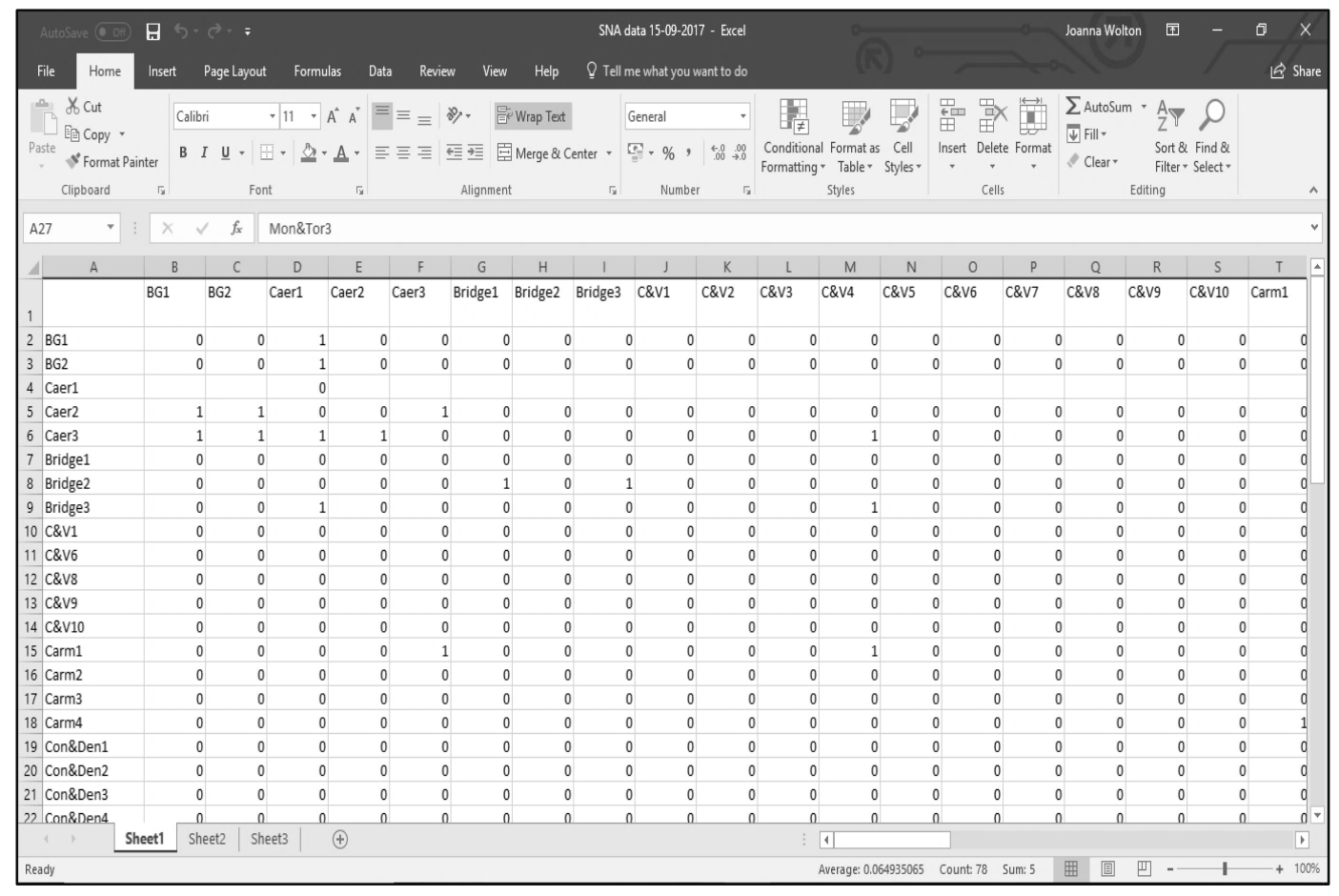




\section{Appendix Item 10- Technical explanation of the normalisation of SNA measures}

The normalised degree centrality measure is calculated by using the degree measure divided by the number of individuals in the network minus 1, then multiplied by one hundred (expressed as a formula below):

Normalised (in or out) Degree Centrality $=\quad$ (In or out) Degree measure $\quad$ x $\quad 100$ (Number of individuals in network-1)

Because this project has directed data (the direction and reciprocity of links can be determined), a normalised measure of outdegree, indegree and betweenness was computed using the above formula. 


\section{Appendix Item 11- Coding of independent variables}

Professional/Vocational qualifications:

Finance: 1 (yes including MLIA DiP, CeMAP) o (no). Health: 1 (yes, which included Trusted Assessor, Macmillan Cancer Support course, nursing training, Chartered Institute of Environmental health, RiPHH, Health and Social Care, Deaf Awareness and Sign Language) o (no). Management: 1 (yes including Higher Diploma in Administration, Prince2-project management, NEBSm, Craft Trainer Award, ILM) o (no). IT: 1 (yes, including European Computer Driving License) o (no). Practical: 1 (yes, including City and Guilds, PGCE, BTEC, Business Studies, Children’s Care and Development, CELTA) o (no). Energy: 1 (yes, including Qualified Domestic Energy Assessor, Home energy efficiency, NEA) o (no). NVQ: 1 (yes) o (no).

\section{Type of training received:}

Health and safety: 1 (yes) o (no). Housing and energy: 1 (yes, including housing adaptation; energy awareness and efficiency) o (no). Finance: 1 (yes) o (no). Personal skills: 1 (yes, including professional skills/ workplace training, interpersonal skills, IT training, IT/ administration) o (no). Interpersonal: 1 (yes, including health training, sensory awareness, and dementia training) o (no).

\section{Previous job sector:}

Public service Healthcare: 1 (yes, including healthcare (non-NHS), NHS, social services) o (no). Public service: 1 (yes, including: education, public administration and uniformed services) o (no). Business: 1 (yes, including banking and finance, business, legal, marketing media and PR, recruitment and HR) O (no). Practical/Technical: 1 (yes, including construction and engineering, creative arts and design, environment and agriculture, IT and technology) o (no). Charity and Voluntary: 1 (yes, including charity and voluntary, volunteer) o (no). Customer Service: 1 (yes, including hospitality, leisure and travel, retail and customer service).

\section{Previous work experience:}

Managerial: 1 (yes, including professional occupations, associate professional and technical occupations) o (no). Administrative: 1 (yes, including administrative and secretarial occupations) o (no). Customer service: 1 (yes, including personal service and sales and customer service) o (no). Practical: 1 (yes, including skilled trade occupation; process, plant and machine operative; elementary occupations) o (no). 
Job title:

Caseworker: 1 (yes, including Caseworkers, Casework Officer and Senior

Caseworkers) o (no). Technical Caseworker: 1 (yes, including Technical Caseworkers and Technical Caseworker Team Leader) o (no). Social care Caseworker: 1 (yes, including Senior Hospital to Home Caseworker, Critical Prevention Caseworker, and RRAP Caseworker) O (no). Specialist Caseworker: 1 (yes, including managing better Caseworker, Hoarding Caseworker and Affordable Warmth Caseworker) o (no).

\section{Core funding:}

From the questionnaire the responses categories were 'yes', 'no' and 'don't know'. For the purposes of analysis, the 'no' and 'don't know responses were recoded into one variable. Once the answers were recoded there were two variables used in the analysis: Yes-funded by Welsh Government: 1 (yes) o (no). Other type offunding: 1 (yes) o (no).

\section{Agency location;}

Blaenau Gwent, 2 (yes) Bridgend 3 (yes), Caerphilly 4 (yes), Cardiff and the Vale 5 (yes), Carmarthenshire 6 (yes), Conwy and Denbighshire 7 (yes), Groynedd a Mon 8 (yes), Merthyr Tydfil 9 (yes), Monmouthshire and Torfaen 10 (yes), Neath Port Talbot 11 (yes), Newport 12 (yes), North East Wales 13 (yes), Powys 14 (yes), Rhondda Cynon Taf 15 (yes), Swansea 16 (yes), West Wales 17 (yes).

\section{Issues on the ground:}

Organisations and services are reluctant to work together: 1 (yes) o (no). Difficulties in accessing clients who live in remote areas: 1 (yes) o (no). ICT problems: 1 (yes) o (no). Client reluctant to accept help: 1 (yes) o (no). Client's objections to giving personal details: 1 (yes) o (no). Client is unhappy about changing their home environment: 1 (yes) o (no). Couples disagree between themselves about the support needed: 1 (yes) o (no). Client's family objects to CER interventions: 1 (yes) o (no). Limited finds available to help support client's needs: 1 (yes) o (no). Changes to benefits eligibility criteria: 1 (yes) o (no). Language barriers: 1 (yes) o (no). Organisations restructuring: 1 (yes) o (no). Time needed to complete administrative tasks: 1 (yes) o (no). 


\section{Appendix Item 12- Portion of the coding matrix for the qualitative interviews wi}

\begin{tabular}{|c|c|c|}
\hline \multicolumn{3}{|l|}{$1:$ P1-Swansea 1} \\
\hline \multicolumn{3}{|l|}{ 1: P1-Swansea 1} \\
\hline $\begin{array}{l}2 \text { : P2-16090503- } \\
\text { _Swansea_2_Transcript }\end{array}$ & $\begin{array}{l}\text { We needed handrails. I've got three little steps out the back. They } \\
\text { put handrails there, handrails in the bathroom, fire alarm things, } \\
\text { and then they put in a new boiler for me. } \\
\text { Like I said, I've had new windows and new doors. I've had a new } \\
\text { roof. They have altered the bathroom for me. The bathroom's } \\
\text { been now altered so that we've got a walk-in shower so that it's } \\
\text { easier for both of us to shower. } \\
\text { As I say, the stair lift was put in. So, the money has made a world } \\
\text { of difference. The house, now, is in reasonably good repair. So, } \\
\text { that's it. }\end{array}$ & $\begin{array}{l}\text { The pressure. Ol } \\
\text { said, she was alw } \\
\text { only had to phon } \\
\text { Amazing. I cann } \\
\text { made life easier } \\
\text { Yes. Oh, I think } \\
\text { amazing people, } \\
\text { last May, then, fr } \\
\text { Everything is so } \\
\text { Can you underst } \\
\text { They took every } \\
\text { off my shoulders }\end{array}$ \\
\hline $\begin{array}{l}\text { 3: P3-16090601- } \\
\text { _Neath_Pt._1_Transcript }\end{array}$ & & $\begin{array}{l}\text { When you live al } \\
\text { you tend to worr } \\
\text { and whatever, bu } \\
\text { and it's there. Th }\end{array}$ \\
\hline $\begin{array}{l}4: \text { : } 3 \text {-16090602- } \\
\text { Neath_Pt.2_Transcript }\end{array}$ & & $\begin{array}{l}\text { Resolution came, } \\
\text { me. }\end{array}$ \\
\hline $\begin{array}{l}5: \text { :P4-16090603- } \\
\text { _Port_Talbot_Transcript }\end{array}$ & & $\begin{array}{l}\text { Although they } \mathrm{h} \\
\text { they put you at } \mathrm{y}\end{array}$ \\
\hline
\end{tabular}






[Occupational Therapist] came out then and said, "Well, we're going to need a grab rail by the toilet, a grab rail somewhere else," and various other different aids around.

Then, she came up with further recommendations of having a shower seat installed so I could go into the shower and sit down, and not trying to balance myself without a stick or anything else in the shower.

A level access all the way through. She put the extra grab rail in the porch way, so there are two there. One at the back of the house, one by the side of my bed, one by the toilet. The raised seat by the toilet, blocks underneath here to raise the settee up so I'm higher up then for access for getting off, or getting up, I should say.

I've had two seats provided. One for in the kitchen for preparing food and washing dishes, and things of that nature, and one in the bathroom, so I can be sitting at the basin to wash, shave and do all things of this nature. They've been set at suitable height, 


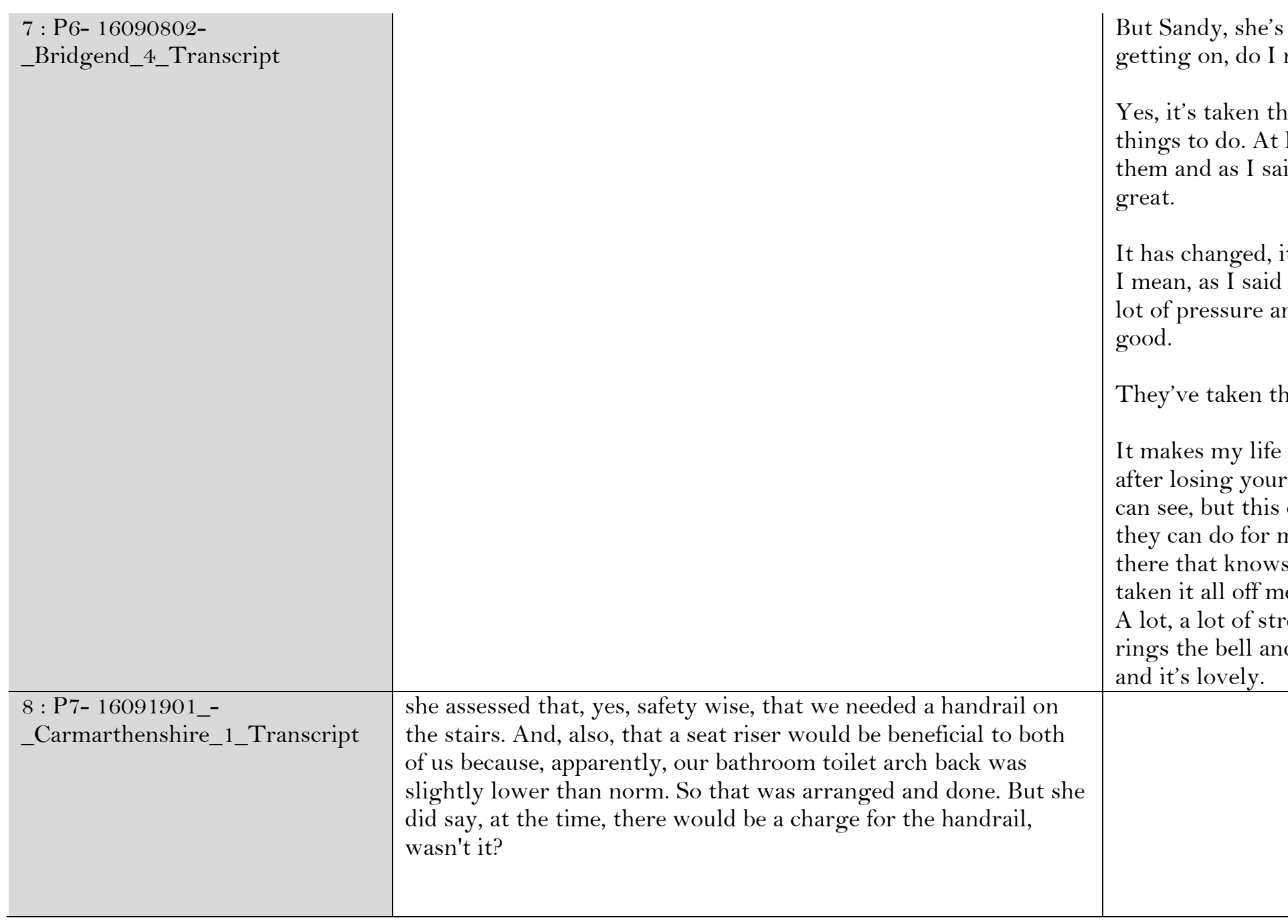




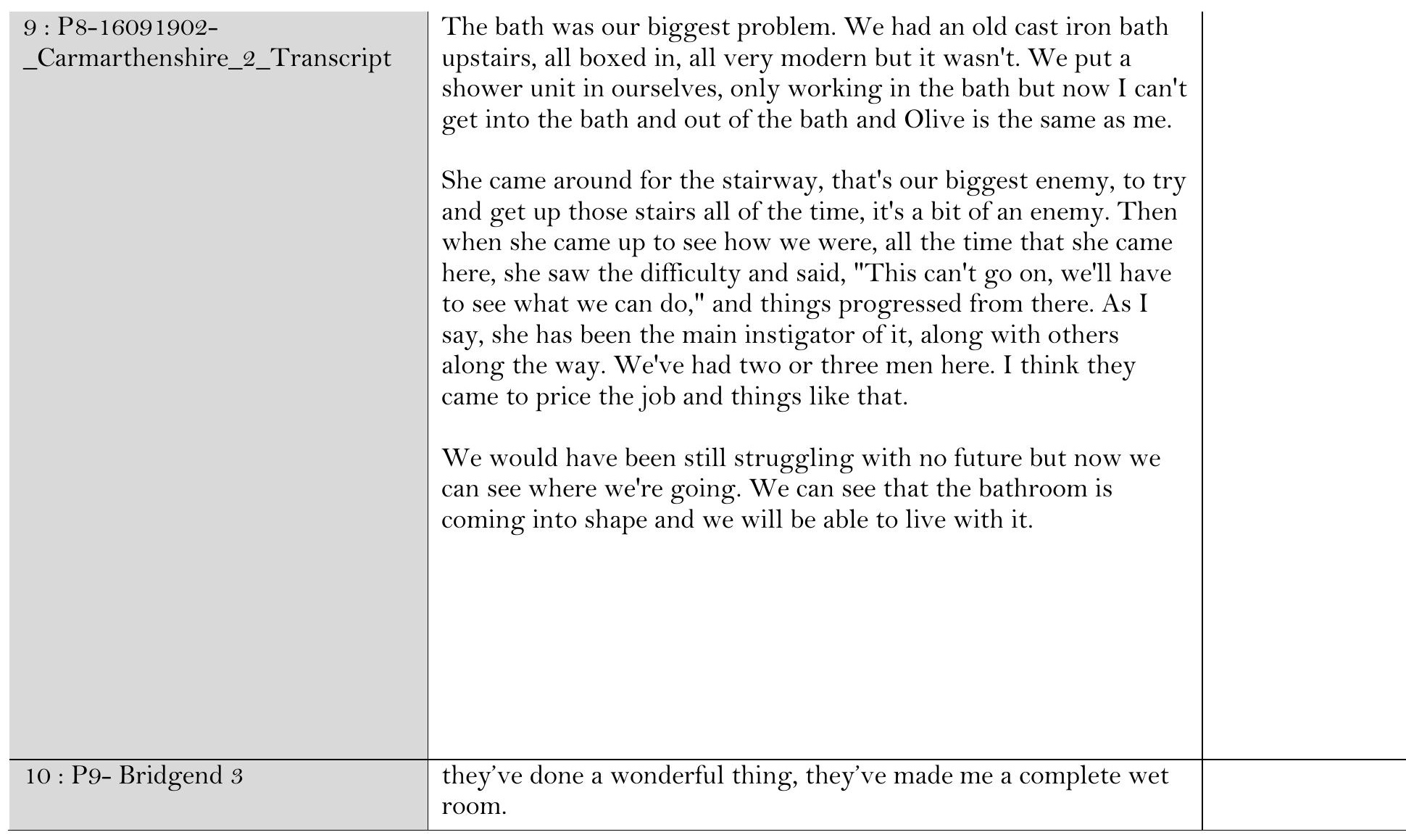


Appendix Item 13- Frequency distribution of capital and socio-demographic characteristics of Caseworkers

Independent Variables Agency Location

- North Wales

- South Wales

- Mid Wales

Gender

- Male

- Female Age

- 20-29

- 30-39

- $40-49$

- $\quad 50-59$

- $60+$

- $\quad$ Missing

Educational qualification

- No qualifications

- GCSE/ O Levels

- Apprenticeship

- Further education/ A Levels or equivalent

- Higher education/ degree level or above Professional/Vocational qualification

- Finance and management

- Health and Social Care$$
\text { - Energy }
$$

- Practical

- NVQ

- None

Training since joining organisation

- Health and Safety

- Housing and Energy

- Finance

- Administrative and Clerical

- Interpersonal Skills (e.g., dementia, mental health, death and loss)

Previous job sectors

Public Service- Healthcare

- Public Service

- Business

- Practical/ Technical

- Charity and Voluntary

- Customer service

Previous work experience

- Managerial

- Administrative

- Practical (e.g. skilled trade occupation, elementary occupations)

- Customer Service

Job Title

- Caseworker (Caseworker, casework officer, senior Caseworker)

- Technical (technical Caseworker and team leader

- Social Care (hospital to home)

- Specialist

Funded by core funding
$\%$ of study population ( $n=55)$

30.9 (response rate $89 \%$ )

63.6 (response rate 65\%)

5.5 (response rate 60\%)

12.7

87.3

9.1

16.7

35.2

35.2

3.7

1.8

1.8

21.8

3.6

32.7

40.0

10.9

16.4

3.6

5.5

18.2

21.8

50.9

70.9

76.4

60.0

61.8

56.4

29.1

56.4

40.0

21.8

43.6

50.9

45.5

63.6

27.3

43.6

74.5

9.1

5.5

10.9

67.3 


\begin{tabular}{r|r}
\hline Length of time in role & \\
- Less than 6 months & 12.7 \\
$-\quad 6$ months - 1 year & 5.5 \\
$-\quad 1-2$ years & 12.7 \\
$-\quad 2-3$ years & 3.6 \\
$-\quad 3-4$ years & 7.3 \\
$-\quad 4-5$ years & 5.5 \\
$-\quad 5+$ years & 52.7 \\
Working hours & \\
$-\quad$ Full time & 74.5 \\
$-\quad$ Part time & 23.6 \\
$-\quad$ Missing & 1.8 \\
Work across several welsh counties $/$ part of & \\
county & \\
$-\quad$ Yes & 65.5 \\
No & 34.5 \\
\hline
\end{tabular}




\section{Appendix Item 14- Centrality scores of non-responders to}

the caseworker questionnaire

\begin{tabular}{|c|c|c|c|c|c|c|}
\hline $\begin{array}{l}\text { No } \\
\text {. }\end{array}$ & $\begin{array}{l}\text { CW } \\
\text { identifier }\end{array}$ & $\begin{array}{l}1 . \\
\text { Outde } \\
\text { gree }\end{array}$ & $\begin{array}{l}. \\
\text { Indegre } \\
\mathrm{e}\end{array}$ & $\begin{array}{l}3 . \\
\text { Normalized } \\
\text { Outdegree }\end{array}$ & $\begin{array}{l}4 . \\
\text { Normalized } \\
\text { Indegree }\end{array}$ & $\begin{array}{l}\text { Total } \\
\text { number } \\
\text { of ties }\end{array}$ \\
\hline 3 & Caer 1 & 0.000 & 4.000 & 0.000 & 0.053 & 4 \\
\hline 10 & $\mathrm{C} \& \mathrm{~V} 2$ & 0.000 & 0.000 & 0.000 & 0.000 & O \\
\hline 11 & $\mathrm{C} \& \mathrm{~V} 3$ & 0.000 & 5.000 & 0.000 & 0.066 & 5 \\
\hline 12 & $\mathrm{C} \& \mathrm{~V} 4$ & 0.000 & 8.000 & 0.000 & 0.105 & 8 \\
\hline 13 & $\mathrm{C} \& \mathrm{~V} 5$ & 0.000 & 1.000 & 0.000 & 0.013 & 1 \\
\hline 15 & $\mathrm{C} \& \mathrm{~V} 7$ & 0.000 & 0.000 & 0.000 & 0.000 & $\mathrm{O}$ \\
\hline 27 & $\begin{array}{l}\text { Gwy\&An } \\
\text { g } 1\end{array}$ & 0.000 & 0.000 & 0.000 & 0.000 & O \\
\hline 31 & $\begin{array}{l}\text { Gwy\&An } \\
\text { g } 5\end{array}$ & 0.000 & 1.000 & 0.000 & 0.013 & 1 \\
\hline 33 & Mon\&Tor & 0.000 & 4.000 & 0.000 & 0.053 & 4 \\
\hline 36 & NPT 1 & 0.000 & 5.000 & 0.000 & 0.066 & 5 \\
\hline 40 & Nport 2 & 0.000 & 1.000 & 0.000 & 0.013 & 1 \\
\hline 41 & Nport 3 & 0.000 & 4.000 & 0.000 & 0.053 & 4 \\
\hline 53 & Pow 3 & 0.000 & 0.000 & 0.000 & 0.000 & O \\
\hline 57 & RCT 3 & 0.000 & 3.000 & 0.000 & 0.039 & 3 \\
\hline 63 & WW 2 & 0.000 & 0.000 & 0.000 & 0.000 & O \\
\hline 64 & WW 3 & 0.000 & 3.000 & 0.000 & 0.039 & 3 \\
\hline 66 & WW 5 & 0.000 & 1.000 & 0.000 & 0.013 & 1 \\
\hline 67 & WW 6 & 0.000 & 0.000 & 0.000 & 0.000 & O \\
\hline 68 & Bridge 4 & 0.000 & 0.000 & 0.000 & 0.000 & $\mathrm{O}$ \\
\hline 69 & Bridge 5 & 0.000 & 6.000 & 0.000 & 0.079 & 6 \\
\hline 70 & Bridge 6 & 0.000 & 1.000 & 0.000 & 0.013 & 1 \\
\hline 77 & Pow 5 & 0.000 & 1.000 & 0.000 & 0.013 & 1 \\
\hline
\end{tabular}




\section{Appendix item 15- Technical explanation of the Latent profile Analysis output}

Once the number of classes (patterns of interaction) had been determined by the latent profile analysis; the output from MPlus indicated the most probable class membership of each Caseworker based on their indegree and outdegree scores. These data were imported into SPSS to explore some of the observed characteristics of these groups based on the most probable class membership (Table 5.9).

Based on probable class membership, Group 1 has greater indegree and outdegree scores compared to group 2 . This can be determined by examining the mean scores of indegree and outdegree, which are 4.08 (SD 1.80) and 10.08 (SD 2.78) respectively for Group 1.

Group 2 tend to have lower scores of indegree which is illustrated by the mean indegree score of 2.24 (SD 2.07), with one exception (Con\&Den 1 who has 10 incoming ties $\left.{ }^{31}\right)$. Additionally, Group 2 also has a low outdegree score with the mean average score of 1.52 (SD 1.80).

${ }^{31}$ Con\&Den 1 was identified as an anomaly during the exploratory analysis of the Caseworkers contained in the different classes. 


\section{References}

Adams, S. (2010). A Perfect Storm: An ageing population, low income home ownership, and decay of older housing. Retrieved from Nottingham: http://careandrepairengland.org.uk/wp-content/uploads/2014/12/A-Perfect-Storm-Nov-10.pdf

Adams, S. (2017). Integration in Action Evaluation of Age UK Warwickshire's provision of impartial information and advice about housing, care and related finance in later life. $\quad$ Retrieved from http://careandrepair-england.org.uk/wpcontent/uploads/2017/05/AgeUk-W-Evaluation-report-Final.pdf

Adams, S. (2018). Small but Significant: The impact and cost benefits of handyperson services. Retrieved from http://careandrepair-england.org.uk/wpcontent/uploads/2018/07/Small-but-Significant-Handyperson-EvaluationCRE-2018.pdf

Adler, P. S., \& Kwon, S. W. (2002). Social capital: Prospects for a new concept. Academy of management review, 27(1), 17-40. doi:10.5465/AMR.2002.5922314

Age Concern and Help the Aged. (2009). One voice: shaping our ageing society, . Retrieved from London: http://www.ageuk.org.uk/documents/en-gb/forprofessionals/research/one\%20voice\%20(2009)_pro.pdf?dtrk=true

Age UK. (2013). Plan to merge NHS and social care budgets. Retrieved from https://www.ageuk.org.uk/latest-news/archive/plan-to-merge-nhs-andsocial-care-budgets/

Age UK Oxfordshire. (2011). Safeguarding the Convoy: A call to action from the Campaign to End Lonliness. Retrieved from

https://www.campaigntoendloneliness.org/wpcontent/uploads/downloads/2011/11/Safeguarding-the-convey.-A-call-toaction-from-the-Campaign-to-End-Loneliness.pdf

Ahlqvist, A., Nyfors, H., \& Suhonen, R. (2016). Factors associated with older people's independent living from the viewpoint of health and functional capacity: a register-based study. Nursing open, 3(2), 79-89.

Allan, H. T. (2006). Using participant observation to immerse oneself in the field: The relevance and importance of ethnography for illuminating the role of emotions in nursing practice. Journal of Research in Nursing, 11(5), 397-407.

Anonymous. (2011). Small investments could make a big difference to older generation. Western Mail.

Aronson, J., \& Neysmith, S. M. (1996). "YOU'RE NOT JUST IN THERE TO DO THE WORK" Depersonalizing Policies and the Exploitation of Home Care Workers' Labor. Gender \& Society, 10(1), 59-77. doi:10.1177/089124396010001005

Asparouhov, T., \& Muthén, B. (2014). Variable-specific entropy contribution. Recuperado de http://www.statmodel.com/download/UnivariateEntropy.pdf.

Auditor General for Wales. (2018). Housing Adaptations. Retrieved from Cardiff: http://www.audit.wales/publication/housing-adaptations

Baez, J. (2016). Network Theory. Online Retrieved from http://math.ucr.edu/home/baez/econ.pdf

Barbour, R. S. (2001). Checklists for improving rigour in qualitative research: a case of the tail wagging the dog? BMJ: British Medical Journal, 322(7294), 1115.

Baumeister, \& Leary. (1997). Writing narrative literature reviews. Review of general psychology, 1(3), 311.

Baxter, J., \& Boyce, S. (2011). Key Issues for the Fourth Assembly. Cardiff Bay: National Assembly for Wales Commission Retrieved from http://www.assembly.wales/NAfW\%20Documents/ki-020.pdf\%20\%2003112011/ki-020-English.pdf.

Becker, G. S. (1962). Investment in human capital: A theoretical analysis. Journal of political economy, 70(5, Part 2), 9-49. 
Becker, G. S. (1994). Human capital revisited. In Human Capital: A Theoretical and Empirical Analysis with Special Reference to Education (3rd Edition) (pp. 15-28): The university of Chicago press.

Beine, M., Docquier, F., \& Rapoport, H. (2008). Brain drain and human capital formation in developing countries: winners and losers. The Economic Journal, $118(528), 631-652$.

Bengston, V., Rosenthal, C., \& Burton, L. (1990). Families and Ageing: diversity and heterogeneity. In R. H. Binstock \& L. K. George (Eds.), Handbook of Aging and the Social Sciences. Third Edition (pp. 263-287). New York: Academic Press.

Benton, T., \& Craib, I. (2011). Philosophy of social science: the philosophical foundations of social thought. Second Edition, 10th Anniversary Edition. Houndsmill, Basingstoke, Hampshire ; New York.: Palgrave Macmillan

Bergen, A. (1992). Case Management in Community Care: Concepts, Practices and Implications for Nursing. Journal of advanced nursing, 17, 1106-1113. doi:http://dx.doi.org/10.1111/j.1365-2648.1992.tb02045.x

Berke, E. M., Koepsell, T. D., Moudon, A. V., Hoskins, R. E., \& Larson, E. B. (2007). Association of the built environment with physical activity and obesity in older persons. American journal of public health, 97(3), 486-492.

Bibbings, J. (2017). The Living Home Standard: how does Wales measure up? Retrieved from https://sheltercymru.org.uk/the-living-home-standardhow-does-wales-measure-up/\#top

Boldy, D., Grenade, L., Lewin, G., Karol, E., \& Burton, E. (2011). Older people's decisions regarding 'ageing in place': A Western Australian case study. Australasian Journal on Ageing, 30(3), 136-142. doi:http://dx.doi.org/10.1111/j.1741-6612.2010.00469.x

Bolton. (2000). Who cares? Offering emotion work as a 'gift'in the nursing labour process. Journal of advanced nursing, 32(3), 580-586.

Bolton. (2001). Changing faces: nurses as emotional jugglers. Sociology of Health $\xi^{2}$ Illness, 23(1), 85-100. doi:10.1111/1467-9566.00242

Bolton. (2005). Emotion management in the workplace: In.

Bone. (2002). Dilemmas of emotion work in nursing under market-driven health care. . The International Journal of Public Sector Management, 15(2), 140-150.

Borgatti, S. P., Everett, M. G., \& Johnson, J. C. (2013). Analyzing social networks: SAGE Publications Limited.

Borgatti, S. P., \& Halgin, D. S. (2011). On network theory. Organization science, 22(5), 1168-1181.

Borgatti, S. P., \& Li, X. (2009). On social network analysis in a supply chain context. Journal of Supply Chain Management, 45(2), 5-22.

Bourdieu, P. (1973). Cultural reproduction and social reproduction. London: Tavistock, 178.

Bourdieu, P., \& Wacquant, L. J. (1992). An invitation to reflexive sociology: University of Chicago press.

Bowles, S., Gintis, H., \& Osborne, M. (2001). The determinants of earnings: A behavioral approach. Journal of economic literature, 39(4), 1137-1176.

Braun, V., \& Clarke, V. (2006). Using thematic analysis in psychology. Qualitative research in psychology, 3(2), 77-101.

Brereton, Kitchenham, Budgen, Turner, \& Khalil. (2007). Lessons from applying the systematic literature review process within the software engineering domain. Journal of systems and software, 80(4), 571-583.

Brook. (2009). In critical defence of 'emotional labour'refuting Bolton's critique of Hochschild's concept. Work, employment छे society, 23(3), 531-548. doi:10.1177/0950017009337071

Bryman, A. (2008). Social research methods (3rd edition ed.). Oxford: Oxford University Press. 
Burgess, G. (2012, June). Housing an ageing population: housing options and the value of information and advice to older people in England. Paper presented at the European Network on Housing Research, Lillehammer, Norway.

Burholt, V. (2006). 'Adref': theoretical contexts of attachment to place for mature and older people in rural North Wales. Environment and Planning A, 38(6), 10951114 .

Burholt, V., Dobbs, C., \& Victor, C. (2016). Transnational relationships and cultural identity of older migrants. GeroPsych.

Burholt, V., \& Sardani, A. V. (2018). The impact of residential immobility and population turnover on the support networks of older people living in rural areas: Evidence from CFAS Wales. Population, Space and Place, 24(4), e2132.

Burnard, P., Gill, P., Stewart, K., Treasure, E., \& Chadwick, B. (2008). Analysing and presenting qualitative data. British dental journal, 204(8), 429.

Burt, R. S. (1997). The contingent value of social capital. Administrative science quarterly, 339-365.

Byrne, B. (2004). Qualitative intervieing. In C. Searle (Ed.), Researching Society and Culture, Second Edition. London: Sage.

Cambridge Intelligence. (2017). KeyLines FAQs: Social Network Analysis. Retrieved from https://cambridge-intelligence.com/keylines-faqs-social-networkanalysis/

Cancian, F. (2000). Paid Emotional Care. In M. H. Meyer (Ed.), Care Work: Gender, Labor, and the Welfare State (pp. 136-148). London, United States: Taylor and Francis.

Care \& Repair Cymru. (2016a). Celebrating 25 Tears of Care \& Repair Cymru. Retrieved from

Cardiff: http://www.careandrepair.org.uk/files/6614/9164/8468/CARE REPAIR _25YEARS.pdf

Care \& Repair Cymru. (2016b). Consult, Respond, Change: Annual Review 2015-2016. Retrieved from Cardiff:

Care \& Repair Cymru. (2017a). About Us- Our Story. Retrieved from http://www.careandrepair.org.uk/en/about-us/our-story/

Care \& Repair Cymru. (2017b). About Us- What we do. Retrieved from http://www.careandrepair.org.uk/en/about-us/what-we-do/

Care \& Repair England. (2013). More than Bricks and Mortar. In. Nottingham: Care \& Repair England.

Care \& Repair North East Wales. (2018). Casework Support Services. Retrieved from http://careandrepairnew.co.uk/our-services/minor-adaptations/

Care\&RepairCymru. (2015). About Care \& Repair Cymru. Retrieved from http://www.careandrepair.org.uk/about/

Carrington, P. J. (2011). Crime and social network analysis. The SAGE handbook of social network analysis, 236-255.

Carter-Davies, L., \& Hillcoat-Nallétamby, S. (2015). Housing for Older People in Wales: An Evidence Review. Retrieved from

Caulfield, J. (2013). A Social Network Analysis of Irish Language Use in Social Media. (PhD ), Cardiff University, Unpublished. Retrieved from http://orca.cf.ac/id/eprint/53228

CGI Group Inc. (2011). Implementing social network analysis for fraud prevention. Retrieved from https://www.cgi.com/sites/default/files/whitepapers/Implementing-social-network-analysis-for-fraud-prevention.pdf

Chan, K., \& Liebowitz, J. (2005). The synergy of social network analysis and knowledge mapping: a case study. International journal of management and decision making, 7(1), 19-35.

Chartered Institute of Environmental Health. (2008). Good Housing Leads to Good Health: A toolkit for environmental health practitioners. Retrieved from London: 
https://www.cieh.org/media/1245/good-housing-leads-to-good-health-atoolkit-for-environmental-health-practitioners.pdf

Chartered Institute of Personnel and Development. (2017). Human capital theory: assessing the evidence for the value and importance of people to organisational success. Technical Report. Retrieved from London: https://www.cipd.co.uk/Images/human-capital-theory-assessing-theevidence_tcm 18-22292.pdf

Chwarae Teg. (2013). A woman's place: A study of women's roles in the Welsh workforcethe full report. Retrieved from https://www.cteg.org.uk/research/womansplace/

Clark, S. L., \& Muthén, B. (2009). Relating latent class analysis results to variables not included in the analysis. In.

Coff, R., \& Kryscynski, D. (2011). Invited editorial: Drilling for micro-foundations of human capital-based competitive advantages. Journal of Management, 37(5), 1429-1443.

Cohen, J. (1999). Trust, voluntary association and workable democracy: the contemporary American discourse of civil society. Democracy and trust, 233, 237.

Coleman. (1988). Social capital in the creation of human capital. American journal of sociology, S95-S120.

Coleman. (1990). Foundations of social theory. Cambridge, MA: Belknap.

Coleman, \& Bond. (1990). Ageing in the Twentieth Century. In J. a. C. Bond, P (Ed.), Ageing in Society: An Introduction to Social Gerontology. London: Sage

Coleman, Katz, \& Menzel. (1957). The diffusion of an innovation among physicians. Sociometry, 253-270. doi:10.2307/2785979

Coleman, Katz, \& Menzel. (1966). Medical innovation: A diffusion study: Bobbs-Merrill Indianapolis.

Colladon, A. F., \& Remondi, E. (2017). Using social network analysis to prevent money laundering. Expert Systems with Applications, 67, 49-58.

Collerton, J., Barrass, K., Bond, J., Eccles, M., Jagger, C., James, O., . . Kirkwood, T. (2007). The Newcastle 85+ study: biological, clinical and psychosocial factors associated with healthy ageing: study protocol. BMC geriatrics, 7(1), 14. doi: $10.1186 / 1471-2318-7-14$

Communities and Local Government Committee. (2018). Housing for older people: Second Report of Session 2017-19. Retrieved from London: https://publications.parliament.uk/pa/cm201719/cmselect/cmcomloc/370 /370.pdf

Coombes, L., Allen, D., \& Neale, J. (2008). In-depth interview. In J. Neale (Ed.), Research Methods for Health and Social Care (pp. 197-2 10). Hampshire: Palgrave Macmillan

Corti, L., Day, A., \& Backhouse, G. (2000). Confidentiality and informed consent: Issues for consideration in the preservation of and provision of access to qualitative data archives. Paper presented at the Forum Qualitative Sozialforschung/Forum: Qualitative Social Research.

Costa-Font, J., Elvira, D., \& Mascarilla-Miró, O. (2009). Ageing in place'? Exploring elderly people's housing preferences in Spain. Urban studies, 46(2), 295-316.

Creswell, J. (2009). Research design: Qualitative, quantitative, and mixed methods approaches (3rd ed.) (Third edition ed.). Thousand Oaks: Sage Publications.

Creswell, J. (2014). Research design : qualitative, quantitative, and mixed methods approaches. Los Angeles, California: Sage.

Crimmins, E. M., \& Beltrán-Sánchez, H. (2011). Mortality and morbidity trends: is there compression of morbidity? The Journals of Gerontology Series B: Psychological Sciences and Social Sciences, 66(1), 75-86. 
Cronin, P., Ryan, F., \& Coughlan, M. (2008). Undertaking a literature review: a stepby-step approach. British journal of nursing, 17(1), 38-43.

Cross, R., Borgatti, S. P., \& Parker, A. (2002). Making Invisible Work Visible: Using Social Network Analysis to Support Strategic Collaboration. California Management Reviere, 44, 25-46.

Crotty, M. (1998). The foundations of social research: Meaning and perspective in the research process. London:: Sage Publications.

Croucher, K., \& Lowson, K. (2011). Handypersons Evalutation: Interim Findings. Retrieved from London: https://assets.publishing.service.gov.uk/government/uploads/system/uplo ads/attachment_data/file/6318/1837939.pdf

Daly, M., \& Silver, H. (2008). Social exclusion and social capital: A comparison and critique. Theory and society, 37(6), 537-566.

Davey, J. (2006). "Ageing in place": the views of older homeowners on maintenance, renovation and adaptation. Social Policy Journal of Nerw Zealand, 27, 128.

Davidson, S. (2012). There's no place like your own Home. Retrieved from http://www.careandrepair.org.uk/theres-no-place-like-your-own-home/

Davison, S. (2012). There's No Place Like Your Own Home: An evaluation of the services of Care $\&$ Repair agencies in Wales. Retrieved from Cardiff:

Dawson, A., Bowes, A., Kelly, F., Velzke, K., \& Ward, R. (2015). Evidence of what works to support and sustain care at home for people with dementia: a literature review with a systematic approach. BMC geriatrics, 15(1), 59.

De Brún, A., \& McAuliffe, E. (2018). Social Network Analysis as a Methodological Approach to Explore Health Systems: A Case Study Exploring Support among Senior Managers/Executives in a Hospital Network. International journal of environmental research and public health, 15(3), 511.

Deaner, B., \& Phillips, D. (2013). Scenarios for the Welsh Government Budget to 202526. Retrieved from London

DeJordy, R., \& Halgin, D. (2008). Introduction to ego network analysis. Boston MA: Boston College and the Winston Center for Leadership E Ethics.

Denzin, N. K. (1978). Triangulation: A case for methodological evaluation and combination. Sociological methods, 339-357.

Department of Health. (2005). Supporting People with Long Term Conditions. An NHS and social care model to support local innovation and integration. Retrieved from London:

www.dh.gov.uk/en/Publicationsandstatistics/Publications/PublicationsPoli cyAndGuidance/DH 4100252

Department of Health. (2014). Comorbidities: a framework of principles for system-wide action. $\quad$ Retrieved from https://assets.publishing.service.gov.uk/government/uploads/system/uplo ads/attachment_data/file/307143/Comorbidities_framework.pdf

Diani, M., \& McAdam, D. (Eds.) (Ed.) (2003). Social movements and networks: Relational approaches to collective action. Oxford: Oxford University Press.

Dupuis-Blanchard, S., Gould, O. N., Gibbons, C., Simard, M., Éthier, S., \& Villalon, L. (2015). Strategies for Aging in Place: The experience of language-minority seniors with loss of independence. Global qualitative nursing research, 2, 2333393614565187.

Edwards, G. (2010). Mixed-method approaches to social network analysis. Retrieved from http://eprints.ncrm.ac.uk/842/1/Social_Network_analysis_Edwards.pdf

Electrical Safety First. (2018). How can we keep older people in Wales Safe? A report by electrical Safety First in association with Swansea University. Retrieved from https://www.electricalsafetyfirst.org.uk/what-we-do/safer-homes-wales/

England, P., \& Farkas, G. (1986). Households. Employment, and Gen. 
Ennis, G., \& West, D. (2010). Exploring the potential of social network analysis in asset-based community development practice and research. Australian Social Work, 63(4), 404-417. doi:10.1080/03 12407X.2010.508167

Ennis, G., \& West, D. (2013). Using social network analysis in community development practice and research: a case study. . Community Development Journal, 48, 40-57. doi:10.1093/cdj/bsso 13

Erickson, R. J. (1993). Reconceptualizing family work: The effect of emotion work on perceptions of marital quality. Journal of Marriage and the Family, 888-900.

Erickson, R. J. (2005). Why emotion work matters: Sex, gender, and the division of household labor. Journal of marriage and family, 67(2), 337-351.

European Commission. (2006). Healthy Ageing-A Challenge for Europe (29). Retrieved from

http://ec.europa.eu/health/ph_projects/2003/action1/docs/2003_1_26_fre p_en.pdf

Evans, G. W. (2003). The built environment and mental health. Journal of urban health, 80(4), 536-555.

Everett, M. G., \& Borgatti, S. P. (2005). Extending centrality. Models and methods in social network analysis, 35(1), 57-76.

Expert Group on Housing an Ageing Population in Wales. (2017). Our Housing AGEnda: meeting the aspirations of older people in Wales. Retrieved from Cardfiff: https://gov.wales/docs/desh/publications/170213-expert-group-finalreport-en.pdf

Fausset, C. B., Kelly, A. J., Rogers, W. A., \& Fisk, A. D. (2011). Challenges to aging in place: Understanding home maintenance difficulties. Journal of Housing for the Elderly, 25(2), 125-141. doi:10.1080/02763893.2011.571105

Feeney, B. C., \& Lemay Jr, E. P. (2012). Surviving relationship threats: The role of emotional capital. Personality and Social Psychology Bulletin, 38(8), 1004-1017.

Fernandez, R. (2007). Alfred Marshall lecture women, work, and culture. Journal of the European Economic Association, 5(2-3), 305-332.

Field. (2003). Social Capital. London: Routledge.

Field. (2009). Discovering Statistics Using SPSS (Third Edition ed.). London, Thousand Oaks, New Delhi, Singapore: Sage.

Fielding, N., \& Thomas, H. (2001). Qualitative interviewing. In N. Gilbert (Ed.), Researching Social Life: Second Edition (pp. 123-145). London: Sage.

Fielding, N., \& Thomas, H. (2008). Qualitative Interviewing. In N. Gilbert (Ed.), Researching Social Life: Third Edition (pp. 245-266). London, Thousand Oaks California, New Dehli, Singapore.: Sage.

Fielo, S. B., \& Warren, S. A. (2001). Home adaptation: Helping older people age in place. Geriatric Nursing, 22(5), 239-246.

Fisk, M. J. (1996). Home truths: issues for housing in Wales: Gomer.

Fisk, M. J., \& Hall, D. (1997). Building our Future: The Housing Challenge for Wales: Institute of Welsh Affairs.

Flap, H., \& Völker, B. (2013). Social Capital. In R. Wittek, T. Snijders, \& V. Nee (Eds.), Handbook Of Rational Choice Social Research (pp. 220-251). Palo Alto, C.A. USA: Stanford Social Sciences.

Fleming, L., \& , \& Waguespack, D. M. (2007). Brokerage, boundary spanning, and leadership in open innovation communities. Organization science, 18, 165-180.

Flick, U. (2006). An introduction to qualitative research: Third Edition. London: Sage Publications.

Friedmann, P. D., Hendrickson, J. C., Gerstein, D. R., \& Zhang, Z. (2004). Designated case managers as facilitators of medical and psychosocial service delivery in addiction treatment programs. The journal of behavioral health services $\mathcal{O}^{\circ}$ research, 31(1), 86-97. 
Fries, J. F. (2002). Aging, natural death, and the compression of morbidity. Bulletin of the World Health Organization, 80, 245-250.

Fries, J. F. (2005). The compression of morbidity. Milbank Quarterly, 83(4), 801-823.

Galaskiewicz, J., \& Wasserman, S. (1994). Introduction: Advances in the Social and Behavioural Sciences from Social Network Analysis. In S. W. J. Galaskiewicz (Ed.), Advances in Social Network Analysis (pp. xiii-3). Thousand Oaks, London: Sage.

Garton, L., Haythornthwaite, C., \& Wellman, B. (1997). Studying online social networks. Journal of Computer-Mediated Communication, 3(1), O-O.

Gendron, B. (2004). Why emotional capital matters in education and in labour? toward an Optimal exploitation of human capital and knowledge management. Retrieved from https://hal.archives-ouvertes.fr/hal-00201223/document

Gillies, V. (2003). Family and intimate relationships: A review of the sociological research. London: Families \& Social Capital Research Group, South Bank University.

Gitlin, L. N. (2003). Conducting research on home environments: Lessons learned and new directions. The Gerontologist, 43(5), 628-637.

Glaeser, E. L., Laibson, D., \& Sacerdote, B. (2002). An economic approach to social capital. The Economic Journal, 112(483).

Glasby, J. (2017). The holy grail of health and social care integration: Cost savings may be hard to identify but the real benefits are human. The British Medical Journal, 356(j801). doi: https://doi.org/10.1136/bmj.j801

Glasby, J., \& Dickinson. (2014). Partnership Working in Health and Social Care: What is Integrated Care and How Can We Deliver It? (Second Edition ed.). Bristol: Policy Press.

Golant, S. M. (2008). Commentary: Irrational exuberance for the aging in place of vulnerable low-income older homeowners. Journal of Aging $\mathscr{E}^{2}$ Social Policy, 20(4), 379-397.

Goodwin, N. (2012, 19/04/2012). 19/04/2012. Retrieved from https://www.kingsfund.org.uk/blog/2012/04/lessons-integrated-carepilots

Gray, B. (2009). The emotional labour of nursing-Defining and managing emotions in nursing work. Nurse Education Today, 29(2), 168-175.

Greenhalgh, Thorne, \& Malterud. (2018). Time to challenge the spurious hierarchy of systematic over narrative reviews? European journal of clinical investigation, $48(6)$, e12931.

Griffiths, S., \& Kippin, H. (2017). Public Services after Austerity: Zombies, Suez or Collaboration? The Political Quarterly.

Grossman, E. R. (2013). An examination of Putnam's, Coleman's, and Bourdieu's conceptualizations of social capital and the structural differences across class, race, and gender groups: The University of Akron.

Grundy, E., \& Henretta, J. C. (2006). Between elderly parents and adult children: a new look at the intergenerational care provided by the 'sandwich generation'. Ageing \& Society, 26(5), 707-722.

Ham, C. (2014). Now is the time to create a combined health and social care system. Retrieved from https://www.kingsfund.org.uk/blog/2014/09/time-createcombined-health-and-social-care-system

Hamza, N., \& Gilroy, R. (2011). The challenge to UK energy policy: An ageing population perspective on energy saving measures and consumption. Energy Policy, 39(2), 782-789.

Handler, S. (2014). A Research and Evalutation Framework for Age Friendly Cities. In. Retrieved from http://hummedia.manchester.ac.uk/institutes/micra/A\%20Research\%20an d\%20Evaluation\%20Framework\%20for\%20Agefriendly\%20Cities web\%20version.pdf 
Hanneman, R. A., \& Riddle, M. (2005). Chapter 10. Centrality and Power. In Introduction to social network methods: University of California Riverside.

Hawe, P., \& Shiell, A. (2000). Social capital and health promotion: a review. Social science E medicine, 51(6), 871-885. doi:10.1016/So277-9536(00)00067-8

Hayes. (2009). Beyond Baron and Kenny: Statistical mediation analysis in the new millennium. Communication monographs, 76(4), 408-420.

Hayes, Mann, Morgan, Kelly, \& Weightman. (2012). Collaboration between local health and local government agencies for health improvement. Cochrane Database of Systematic Reviews, 10, 1-138.

Haynes, P. (2009). Before Going Any Further With Social Capital: Eight Key Criticisms to Address. Retrieved from http://digital.csic.es/bitstream/10261/14203/1/Before Going Any Furth er_With_Social_Capital_Eight_Key_Criticisms_to_Address\%5B1\%5D.pdf

Haynes, P. (2014). A Framework to Address the Key Problems of Social Capital. In: Royal Holloway University.

Healy, J. D., \& Clinch, J. P. (2004). Quantifying the severity of fuel poverty, its relationship with poor housing and reasons for non-investment in energysaving measures in Ireland. Energy Policy, 32(2), 207-220.

Hébert, R. (1997). Functional decline in old age. Canadian Medical Association Journal, $157(8), 1037-1045$.

Heywood, F., Oldman, C., \& Means, R. (2002). Housing and Home in Later Life. Milton Keynes: Open University Press.

Hillcoat-Nallétamby, S. (2014). The Meaning of "Independence" for Older People in Different Residential Settings. The Journals of Gerontology Series B: Psychological Sciences and Social Sciences, 69(3), 419-430. doi:10.1093/geronb/gbuoo8

Hillcoat-Nalletamby, S., \& Ogg, J. (2014). Moving beyond 'ageing in place': older people's dislikes about their home and neighbourhood environments as a motive for wishing to move. Ageing \& Society, 34(10), 1771-1796.

Hillcoat-Nalletamby, S., \& Sardani, A. (2015). Preliminary Research Evaluation Report. Moving On: Qualitative Service Evaluation. In. Porthmadog: Canllaw.

Hjermstad, Fayers, Haugen, Caraceni, Hanks, Loge, ... Collaborative, E. P. C. R. (2011). Studies comparing Numerical Rating Scales, Verbal Rating Scales, and Visual Analogue Scales for assessment of pain intensity in adults: a systematic literature review. Journal of pain and symptom management, 41(6), 1073-1093.

Hochschild, A. R. (2003). The managed heart: commercialization of human feeling (20th edition ed.). Berkeley, California: University of California Press.

Hoff. (2015). Current and future challanges of family care in the UK: Future of an ageing population: evidence review. Retrieved from London: http://dera.ioe.ac.uk/24148/1/gs-15-18-future-ageing-family-care-er09.pdf

Holloway, I., \& Wheeler, S. (2002). Qualitative Research in Nursing (Second Edition ed.). Oxford: Blackwell.

Hoover, R. S., \& Koerber, A. L. (2011). Using NVivo to answer the challenges of qualitative research in professional communication: Benefits and best practices tutorial. IEEE transactions on Professional Communication, 54(1), 6882.

Howden-Chapman. (2004). Housing standards: a glossary of housing and health. Journal of Epidemiology \& Community Health, 58(3), 162-168.

Howden-Chapman, Signal, \& Crane. (1999). Housing and health in older people: ageing in place. Social Policy Journal of New Zealand, 14-30. 
Howe, K. R. (1988). Against the Quantitative- Qualitative Incompatilibity Thesis or Dogmas Die Hard. Educational researcher, 17(8), 10-16. doi: 10.3102/0013189X017008010

Hülsheger, U. R., \& Schewe, A. F. (2011). On the costs and benefits of emotional labor: A meta-analysis of three decades of research. Journal of occupational health psychology, 16(3), 361.

Hwang, E., Cummings, L., Sixsmith, A., \& Sixsmith, J. (2011). Impacts of home modifications on aging-in-place. Journal of Housing for the Elderly, 25(3), 246257.

Inkpen, A. C., \& Tsang, E. W. (2005). Social capital, networks, and knowledge transfer. Academy of management review, 30(1), 146-165. doi:10.5465/AMR.2005.15281445

Institute for Fiscal Studies. (2017). Recent cuts to public spending. Retrieved from https://www.ifs.org.uk/tools_and_resources/fiscal_facts/public_spending_ survey/cuts_to_public_spending

IpsosMORI, Shelter, \& British Gas. (2017). Living Home Standard. Retrieved from https://sheltercymru.org.uk/wp-content/uploads/2015/02/Living-HomeStandard-Wales-FINAL-ENG.pdf

Israelsen, R. D., \& Yonker, S. E. (2017). Key human capital. Journal of Financial and Quantitative Analysis, 52(1), 175-214.

Ivers, R., Cumming, R., \& Mitchell, P. (2002). Poor vision and risk of falls and fractures in older Australians: the Blue Mountains Eye Study. New South Wales public health bulletin, 13(2), 8-10.

Jahan, N., Naveed, S., Zeshan, M., \& Tahir, M. A. (2016). How to conduct a systematic review: a narrative literature review. Cureus, $8(11)$.

Jamshed, S. (2014). Qualitative research method-interviewing and observation. Journal of basic and clinical pharmacy, 5(4), 87.

Joffe, H. (2012). Thematic analysis. In Harper \& Thompson (Eds.), Qualitative research methods in mental health and psychotherapy: A guide for students and practitioners (Vol. 1, pp. 219-223). Chichester: Wiley-Blackwell.

Johansson, K., Josephsson, S., \& Lilja, M. (2009). Creating possibilities for action in the presence of environmental barriers in the process of 'ageing in place'. Ageing \& Society, 29(1), 49-70.

Johnson, R. B., \& Onwuegbuzie, A. J. (2004). Mixed methods research: A research paradigm whose time has come. Educational researcher, 33(7), 14-26. doi:10.3102/0013189X033007014

Johnson, R. B., Onwuegbuzie, A. J., \& Turner, L. A. (2007). Toward a definition of mixed methods research. Journal of mixed methods research, 1(2), 112-133.

Joseph Rowntree Foundation. (1997). The state of UK housing. Retrieved from York: file://tawe dfs/Students/6/836106/Downloads/h209\%20(1).pdf

Kannisto, Koivunen, \& Välimäki. (2014). Use of mobile phone text message reminders in health care services: a narrative literature review. Journal of medical Internet research, 16(10).

Kidd, P. S., \& Parshall, M. B. (2000). Getting the focus and the group: enhancing analytical rigor in focus group research. Qualitative health research, 10(3), 293308.

Kitzinger, J. (1995). Qualitative research. Introducing focus groups. BMJ: British medical journal, 311 (7000), 299.

Klein, B. E., Moss, S. E., Klein, R., Lee, K. E., \& Cruickshanks, K. J. (2003). Associations of visual function with physical outcomes and limitations 5 years later in an older population: the Beaver Dam eye study. Ophthalmology, 110(4), 644-650. 
Krieger, J., \& Higgins, D. L. (2002). Housing and Health: Time Again for Public Health Action. American journal of public health, 92(5), 758-768. doi: 10.2 105/ajph.92.5.758

Krothe, J. S. (1997). Giving voice to elderly people: Community-based long-term care. Public Health Nursing, 14(4), 217-226.

Kruskal, C. P., \& Snir, M. (1986). A unified theory of interconnection network structure. Theoretical Computer Science, 48, 75-94.

Laumann, E. O., Marsden, P. V., \& Prensky, D. (1989). The boundary specification problem in network analysis. Research methods in social network analysis, 61, 87.

Lawton, M. P. (1982). Competence, environmental press, and the adaptations of older people. In W. Lawton, and Byerts (Ed.), Aging and the Environment: Theoretical Approaches (pp. 97-120). New York: Springer.

Leather, P., \& Morrison, T. (1997). The State of UK Housing: A factfile on dwelling conditions: Policy Press Bristol.

Leech, N. L., \& Onwuegbuzie, A. J. (2009). A typology of mixed methods research designs. Quality \& Quantity, 43(2), 265-275. doi:10.1007/s $11135-007-9105-3$

Leeson, G. W., Harper, S., \& Levin, S. (2004). Independent living in later life: Literature review: DWP.

Leidner, R. (1993). Fast food, fast talk: Service work and the routinization of everyday life: University of California Press.

Leslie, K., \& Canwell, A. (2010). Leadership at all levels: Leading public sector organisations in an age of austerity. European Management Journal, 28(4), 297305.

Liebowitz, J. (2005). Linking social network analysis with the analytic hierarchy process for knowledge mapping in organizations. Journal of knowledge management, 9(1), 76-86.

Lin. (1999). Building a network theory of social capital. Connections, 22(1), 28-51.

Lin. (2001). Social Capital: A Theory of Structure and Action. New York: Cambridge University Press.

Lin, \& Chiou. (2011). Social Brokerage behind Knowledge Sharing. Paper presented at the Advances in Social Networks Analysis and Mining (ASONAM), 2011 International Conference on.

Lloyd, E., McCormack, C., McKeever, M., \& Syme, M. (2008). The effect of improving the thermal quality of cold housing on blood pressure and general health: a research note. Journal of Epidemiology \& Community Health, 62(9), 793-797.

Long, Cunningham, \& Braithwaite. (2013). Bridges, brokers and boundary spanners in collaborative networks: a systematic review. BMC health services research, 13(1), 158. doi:10.1186/1472-6963-13-158.

Long, Cunningham, Carswell, \& Braithwaite. (2013). Who are the key players in a new translational research network?. BMC health services research, 13(1), 1-11. doi:10.1186/1472-6963-13-338.

Lopez, S. H. (2006). Emotional labor and organized emotional care: Conceptualizing nursing home care work. Work and occupations, 33(2), 133-160.

Lowndes, V., \& Squires, S. (2012). Cuts, collaboration and creativity. Public Money \& Management, 32(6), 401-408.

Luchinskaya, Ogle, \& Trickey. (2017). A delicate balance? Health and Social Care spending in Wales from http://www.walespublicservices2025.org.uk/files/2017/03/Wales-healthand-social-care-final_amended_04-2017.pdf

Luke, D. A., \& , \& Harris, J. K. (2007). Network analysis in public health: history, methods, and applications. The Annual Review of Public Health, 28, 69-93. doi:10.1146/annurev.publhealth.28.021406.144132

Mackintosh, S., \& Leather, P. (2016). The Disabled Facilities Grant: Before and after the introduction of the Better Care Fund. Retrieved from http://careandrepair- 
england.org.uk/wp-content/uploads/2014/12/dfg-report-final-interactiveconverted-draft-6-small.pdf

Manini, T. M. (2013). Mobility decline in old age: a time to intervene. Exercise and sport sciences reviews, $41(1), 2$.

Marin, A., \& Wellman, B. (2011). Social network analysis: An introduction. In J. S. \& \& P. J. Carrington (Eds.), The SAGE handbook of social network analysis (pp. 1125). London: Sage.

Marsden, P. (1998). Memetics and social contagion: Two sides of the same coin. Journal of Memetics-Evolutionary Models of Information Transmission, 2(2), 171185.

Mason. (2010). Sample size and saturation in PhD studies using qualitative interviews. Paper presented at the Forum qualitative Sozialforschung/Forum: qualitative social research.

Mason, \& Roys. (2011). The Health Costs of Cold Dwellings. Retrieved from Garston: https://www.cieh.org/media/1380/the-health-costs-of-cold-dwellings.pdf

Maxim, P. S. (1999). Quantiative Research Methods in the Social Sciences. Oxford: Oxford University Press.

McIllwain, J. S. (1999). Organized crime: A social network approach. Crime, Law and Social Change, 32(4), 301-323.

McLachlan, G., \& Peel, D. (2000). Mixtures of factor analyzers. Finite Mixture Models, 238-256.

McQuaid, R. W. (2000). The theory of partnership: why have partnerships? Routledge Advances in Management and Business Studies, 19, 9-35.

McQueen, A. C. (2004). Emotional intelligence in nursing work. Journal of advanced nursing, 47(1), 101-108.

Means, R. (2007). Safe as houses? Ageing in place and vulnerable older people in the UK. Social Policy \& Administration, 41(1), 65-85. doi:10.1111/j.14679515.2007.00539.x

Meltzer, D., Chung, J., Khalili, P., Marlow, E., Arora, V., Schumock, G., \& Burt, R. (2010). Exploring the use of social network methods in designing healthcare quality improvement teams. Social science \& medicine, 71(6), 1119-1130. doi:10.1016/j.socscimed.2010.05.012

Merton, R. K., Fiske, M., \& Kendall, P. L. (1956). The Focused Interview: A Manual of Problems and Procedures. New York: Free Press.

Meyer, M. (2010). The Rise of the Knowledge Broker. Science Communication, 32(1), 118-127. doi:10.1177/1075547009359797

Mislove, A., Koppula, H. S., Gummadi, K. P., Druschel, P., \& Bhattacharjee, B. (2008). Growth of the flickr social network. Paper presented at the Proceedings of the first workshop on Online social networks.

Mislove, A., Marcon, M., Gummadi, K. P., Druschel, P., \& Bhattacharjee, B. (2007). Measurement and analysis of online social networks. Paper presented at the Proceedings of the 7th ACM SIGCOMM conference on Internet measurement.

Mobius, M., Quoc-Anh, D., \& Rosenblat, T. S. (2004). Social capital in social networks. Retrieved March, 3, 2009.

Moody, H. R. (2010). Aging: Concepts and Controversies (6th Edition ed.). Los Angeles, London, New Dehli, Singapore, Washington: Pine Forge Press.

Morgan, D. L. (1996). Focus groups. Annual review of sociology, 22(1), 129-152.

Moriarty, J., Manthorpe, J., \& Cornes, M. (2015). Reaching out or missing out: approaches to outreach with family carers in social care organisations. Health $E^{2}$ social care in the community, 23(1), 42-50.

Moses, J. W., \& Knutsen, T. L. (2012). Ways of knowing : competing methodologies in social and political research (Second Edition). New York, NY Palgrave Macmillan. 
Mur-Veeman, I., Hardy, B., Steenbergen, M., \& Wistow, G. (2003). Development of integrated care in England and the Netherlands: managing across publicprivate boundaries. Health policy, 65(3), 227-241.

Musselwhite, C. (2011). Successfully giving up driving for older people. Retrieved from London: http://eprints.uwe.ac.uk/16246

Mynatt, E. D., Melenhorst, A.-S., Fisk, A.-D., \& Rogers, W. A. (2004). Aware technologies for aging in place: understanding user needs and attitudes. IEEE Pervasive Computing, 3(2), 36-41.

Nahapiet, \& Ghoshal. (2000). Social capital, intellectual capital, and the organizational advantage. In Knowledge and social capital (pp. 119-157): Elsevier.

National Assembly for Wales. (2001). Welsh House Condition Survey 1998. Cardiff: National Assembly for Wales.

National Assembly for Wales Public Accounts Committee. (2018). The Welsh Government's Supporting People Programme. Retrieved from Cardiff Bay: https://www.assembly.wales/laid\%20documents/cr-ld 11557/cr-ld11557e.pdf

National Institute of Aging. (2010). Global Health and Aging. Retrieved from http://www.who.int/ageing/publications/global health/en/

Newman, M. E. (2005). A measure of betweenness centrality based on random walks. Social networks, 27(1), 39-54.

NHS. (2018, 10/01/2018). What is the Mental Capacity Act? Retrieved from https://www.nhs.uk/conditions/social-care-and-support/mental-capacity/

Nicaise, P., Tulloch, S., Vincent, D., Matanov, A., Priebe, S., \& Lorant, V. (2012). Using Social Network Analysis for Assessing Mental Health and Social Services Inter-organisational Collaboration: Findings in Deprived Areas in Brussels and London. Administration and Policy in Mental Health and Mental Health Services Resaerch, 40, 331-339. doi:10.1007/s 10488-012-0423-y.

Nowotny, H. (1981). Women in public life in Austria. In C. F. Epstein \& C. R.L. (Eds.), Access to power: Cross-national studies of women and elites (pp. 145-158). London: George Allen \& Unwin.

Oberski, D. (2016). Mixture models: Latent profile and latent class analysis. In Modern Statistical Methods for HCI (pp. 275-287): Springer.

Office for National Statistics. (2011). DC2403WA - Welsh language skills by tenure by age - Household Reference Persons. Retrieved from https://www.nomisweb.co.uk/census/2011/DC2403WA/view/209295770

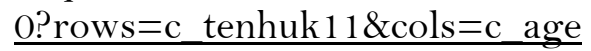

Office for National Statistics. (2013). Characteristics of Older People: What does the 2011 Census tell us about the "oldest old" living in England \& Wales? Retrieved from https://www.ons.gov.uk/peoplepopulationandcommunity/birthsdeathsand marriages/ageing/articles/characteristicsofolderpeople/2013-12o6\# general-health

Office for National Statistics. (2015). National population projections, 2014- based Statistical Bulletin. Retrieved from http://www.ons.gov.uk/ons/dcp171778_420462.pdf

Office for National Statistics. (2016). Families and households in the UK: 2016. Retrieved from https://www.ons.gov.uk/peoplepopulationandcommunity/birthsdeathsand marriages/families/bulletins/familiesandhouseholds/2016

Office for National Statistics. (2017a). Births in England and Wales: 2017. Retrieved from

https://www.ons.gov.uk/peoplepopulationandcommunity/birthsdeathsand marriages/livebirths/bulletins/birthsummarytablesenglandandwales/2017 \#the-number-of-live-births-and-the-total-fertility-rate-decreased-in-2017 
Office for National Statistics. (2017b). Estimates of the Very Old (including Centenarians): 2002 to 2016: People in the oldest age groups in the UK (90 to 104 and 105 and over) by sex and age, and comparisons of the oldest populations in the 4 constituent countries of the UK. Retrieved from https://www.ons.gov.uk/peoplepopulationandcommunity/birthsdeathsand marriages/ageing/bulletins/estimatesoftheveryoldincludingcentenarians/2 002to2016\#main-points

Oldman, J. (2014). Housing in Later Life. Retrieved from https://www.ageuk.org.uk/globalassets/age-uk/documents/reports-andpublications/reports-and-briefings/safe-at$\underline{\text { home/rb_july 14_housing_later_life_report.pdf }}$

ONS. (2012). 2011 Census- Population and Household Estimates for Wales, March 2011. Retrieved from Online: http://www.ons.gov.uk/ons/rel/census/2011census/population-and-household-estimates-for-wales/stb-2011-censuswales.html

ONS. (2014). Changes in the Older Resident Care Home Population between 2001 and 2011. Retrieved from London:

Oswald, F., Jopp, D., Rott, C., \& Wahl, H.-W. (2011). Is aging in place a resource for or risk to life satisfaction? The Gerontologist, 51(2), 238-250. doi:10.1093/geront/gnq096

Otte, E., \& Rousseau, R. (2002). Social network analysis: a powerful strategy, also for the information sciences. Journal of information Science, 28(6), 441-453.

Peace, S., Holland, C., \& Kellaher, L. (2011). 'Option recognition'in later life: variations in ageing in place. Ageing \& Society, 31(5), 734-757.

Phillips, J. E., Ajrouch, K. J., \& Hillcoat-Nallétamby, S. (2010). Key concepts in social gerontology. London, Thousand Oaks, New Dehli, Singapore, Washington D.C: Sage.

Piketty, T. (2014). Capital in the 21st century. Cambridge, MA: Belknap Harvard University Press.

Pisaniello, S. L., Winefield, H. R., \& Delfabbro, P. H. (2012). The influence of emotional labour and emotion work on the occupational health and well-being of South Australian hospital nurses. Journal of Vocational Behavior, 80, 579591. doi:10.1016/j.jvb.2012.02.015.

Portes, A. (1998). Social Capital: Its Origins and Applications in Modern Sociology. Annu. Rev. Sociol, 24, 1-24. doi:10.1146/annurev.soc.24.1.1

Pow, J., Gayen, K., Elliott, L., \& Raeside, R. (2012). Understanding complex interactions using social network analysis. Journal of clinical nursing, 21(19pt20), 2772-2779. doi:http://dx.doi.org/10.1111/j.13652702.2011.04036.x

Powell, J., Mackintosh, S., Bird, E., Ige, J., Garrett, H., \& Roys, M. (2017). The role of home adaptations in improving later life. Retrieved from Bristol: http://eprints.uwe.ac.uk/33945/1/The\%20role\%20of\%20home\%20adaptati ons\%20in\%20improving\%20later\%20life_Full\%20report.pdf

Procter, R., Greenhalgh, T., Wherton, J., Sugarhood, P., Rouncefield, M., \& Hinder, S. (2014). The day-to-day co-production of ageing in place. Computer Supported Cooperative Work (CSCW), 23(3), 245-267.

Provan, \& Milward. (2001). Do networks really work? A framework for evaluating public-sector organizational networks. Public administration review, 61(4), 414-423.

Provan, Veazie, Staten, \& Teufel-Shone. (2005). The Use of Network Analysis to Strengthen Community Partnerships. Public Administration Review, 65, 603614. doi:10.1111/j.1540-62 10.2005.00487.x

Putnam, R. D. (2000). Bowling alone: America's declining social capital. In Culture and politics (pp. 223-234): Springer. 
Putnam, R. D., Leonardi, R., \& Nanetti, R. Y. (1994). Making democracy work: Civic traditions in modern Italy: Princeton university press.

Quadri, P., Tettamanti, M., Bernasconi, S., Trento, F., \& Loew, F. (2005). Lower limb function as predictor of falls and loss of mobility with social repercussions one year after discharge among elderly inpatients. Aging clinical and experimental research, $17(2), 82-89$.

Rabiee, P. (2013). Exploring the relationships between choice and independence: experiences of disabled and older people. British Journal of Social Work, 43(5), 872-888. doi:10.1093/bjsw/bcs022

Rafaeli, A., \& Sutton, R. I. (1987). Expression of emotion as part of the work role. Academy of management review, 12(1), 23-37.

Rainer, H., \& Siedler, T. (2005). O brother, where art thou? The effects of having a sibling on geographic mobility and labor market outcomes. Institute for social and Economic Research Working Paper Series 2005-18.

RAND Europe, \& Ernst \& Young. (2012). National Evaluation of the Department of Health's Integrated Care Pilots Retrieved from Cambridge, UK: https://assets.publishing.service.gov.uk/government/uploads/system/uplo ads/attachment_data/file/215103/dh_133127.pdf

Rantakokko, Mänty, \& Rantanen. (2013). Mobility Decline in Old Age. Exercise and Sport Sciences Review, 41(1), 19-25.

Ray, C. T., Horvat, M., Croce, R., Mason, R. C., \& Wolf, S. L. (2008). The impact of vision loss on postural stability and balance strategies in individuals with profound vision loss. Gait E posture, 28(1), 58-61.

Roberts, M. S. (2014). The influence of lifestyle on the association between health conditions and cognitive function in older people : analysis of CFAS Wales Interim Data. (PhD Thesis), Swansea, Swansea

Robson, C. (2003). Real World Research, 2nd Edition. Oxford: Blackwell.

Roebuck, J. (1979). When does" old age begin?: The evolution of the English definition. Journal of Social History, 12(3), 416-428.

Rogers, A., Brooks, H., Vassilev, I., Kennedy, A., Blickem, C., \& Reeves, D. (2014). Why less may be more: a mixed methods study of the work and relatedness of 'weak ties' in supporting long-term condition self-management. Implementation Science, 9(1), 19.

Ross, Curry, \& Goodwin. (2011). Case management: What it is and how it can best be implemented. In. London: The King's Fund.

Roys, M., Davidson, M., Nicol, S., Ormandy, D., \& Ambrose, P. (2010). The real cost of poor housing. Retrieved from Watford: https://www.hud.gov/sites/documents/REAL_COST_POOR_HOUSING. $\underline{\mathrm{PDF}}$

Rudge, J., \& Gilchrist, R. (2005). Excess winter morbidity among older people at risk of cold homes: a population-based study in a London borough. Journal of Public Health, 27(4), 353-358.

Saldaña, J. (2015). The coding manual for qualitative researchers: Sage.

Sarantakos, S. (1998). Social research, 2nd Edition. Basingstoke: Macmillan.

Sarantakos, S. (2005). Social Research (Third Edition). Houndmills, Basingstoke, Hampshire: Palgrave Macmillan.

Sarantakos, S. (2013). Social Research 4th Edition (4th ed.). London: Palgrave Macmillan.

Schoeni, R. F., \& Blank, R. M. (2000). What has welfare reform accomplished? Impacts on welfare participation, employment, income, poverty, and family structure. Retrieved from California: http://www.dtic.mil/dtic/tr/fulltext/u2/a376640.pdf

Schultz, T. W. (1961). Investment in human capital. The American economic reviere, 51(1), 1-17.

Scott, J. (1991). Social Network Analysis: A Handbook. London: Sage. 
Secker, J., Hill, R., Villeneau, L., \& Parkman, S. (2003). Promoting independence: but promoting what and how? Ageing \& Society, 23(3), 375-391.

Seed, P. (1990). Introducing Network Analysis in Social Work. London: Jessica Kingsley Publishers.

Seidel, D., Richardson, K., Crilly, N., Matthews, F. E., Clarkson, P. J., \& Brayne, C. (2010). Design for independent living: activity demands and capabilities of older people. Ageing \& Society, 30(7), 1239-1255.

Shergold, I., \& Parkhurst, G. (2012). Transport-related social exclusion amongst older people in rural Southwest England and Wales. Journal of Rural Studies, 28(4), 412-421.

Sibbald, S. L., Wathen, C. N., Kothari, A., \& Day, A. M. (2013). Knowledge flow and exchange in interdisciplinary primary health care teams (PHCTs): an exploratory study. Journal of the Medical Library Association: JMLA, 101(2), 128-137. doi:10.3163/1536-5050.101.2.008

Siisiainen, M. (2003). Two concepts of social capital: Bourdieu vs. Putnam. International Journal of Contemporary Sociology, 4O(2), 183-204.

Sixsmith, A., \& Sixsmith, J. (2008). Ageing in place in the United Kingdom. Ageing international, 32(3), $219-235$.

Small, B. J., Dixon, R. A., \& McArdle, J. J. (2011). Tracking cognition-health changes from 55 to 95 years of age. Journals of Gerontology Series B: Psychological Sciences and Social Sciences, 66(suppl_1), i153-i161.

Smith, Bakker, Leenders, Gabbay, Kratzer, \& Van Engelen. (2006). Is trust really social capital? Knowledge sharing in product development projects. The Learning Organization, 13(6), 594-605.

Smith, Flowers, \& Larkin. (2009). Interpretative Phenomenological Analysis: Theory, Method and Research. . London, Thousand Oaks, New Dehli, Singapore: SAGE Publications.

Social Research Association. (2013). Ethical Guidelines. In: Social Research Association.

Spengler, J. (1977). Adam Smith on Human Capital. The American Economic Reviere, $67(1), 32-36$.

StatsWales. (2017a, April 2017). General Fertily rates \& Total Fertility rates by year (1971 onwards) Retrieved from https://statswales.gov.wales/Catalogue/Health-and-Social-Care/BirthsDeaths-and-Conceptions/Births/totalfertilityrateandgeneralfertilityrate-byyear

StatsWales. (2017b, 26-09-2017). Household Estimates by Local Authority, Wales, 1991 to 2016 Retrieved from https://statswales.gov.wales/Catalogue/Housing/Households/Estimates/a veragehouseholdsize-by-localauthority-year

Stephenson, K. (2005). Trafficking in trust: The Art and Science in Human Knowledge Networks. In Couglin, Wingard, \& Hollihan (Eds.), Enlightened Power: How Women are Transforming the Practice of Leadership. San Francisco, CA.: Jossey-Bass.

Stones, D., \& Gullifer, J. (2016). 'At home it's just so much easier to be yourself': older adults' perceptions of ageing in place. Ageing $\mathcal{E}^{2}$ Society, 36(3), 449-481.

Strazdins, L. M. (2000). Integrating emotions: Multiple role measurement of emotional work. Australian Journal of Psychology, 52(1), 41-50.

Sweetland, S. R. (1996). Human capital theory: Foundations of a field of inquiry. Review of Educational Research, 66(3), 341-359.

Tan, E. (2014). Human capital theory: A holistic criticism. Review of Educational Research, 84(3), 411-445.

Tashakkori, A., ., \& Teddlie, C. (1998). Mixed methodology : combining qualitative and quantitative approaches. Thousand Oaks, California: Sage. 
Tein, J.-Y., Coxe, S., \& Cham, H. (2013). Statistical power to detect the correct number of classes in latent profile analysis. Structural equation modeling: a multidisciplinary journal, 20(4), 640-657.

Terry, L. (2017). Producing the Goods: Collaboration as the next frontier of productivity. Retrieved from London: http://www.nlgn.org.uk/public/wpcontent/uploads/Producing-the-Goods_Final.pdf

The National Archives. (2016). SOC2010 volume 3: the National Statistics Socioeconomic classification (NS-SEC rebased on SOC2010). Retrieved from http://webarchive.nationalarchives.gov.uk/20160105160709/http://www.o ns.gov.uk/ons/guide-method/classifications/current-standardclassifications/soc2010/soc2010-volume-3-ns-sec--rebased-on-soc2010-user-manual/index.html

Theodosius, C. (2008). Emotional labour in health care: The unmanaged heart of nursing. London; New York: Routledge.

Tichy, N. M., Tushman, M. L., \& Fombrun, C. (1979). Social Network Analysis for Organizations. Academy of management review, 4, 507-5 19.

Timonen, V., \& Doyle, M. (2008). From the workhouse to the home: evolution of care policy for older people in Ireland. International Journal of Sociology and Social Policy, 28(3/4), 76-89.

Tinker. (1997). Housing for elderly people. Reviews in Clinical Gerontology, 7(2), 171 176.

Tinker, Wright, McCreadie, Askham, Hancock, \& Holmans. (1999). Alternative Models of Care for Older People. Report for the Royal Commission on Long-term Care. Research Volume Two. Retrieved from London:

Tomassini. (2005). The demographic characteristics of the oldest old in the United Kingdom. Population Trends, 120(Summer), 15-22.

Treweek. (1996). Emotion work, order, and emotional power in care assistant work. In V. James \& J. Gabe (Eds.), Sociology of health and illness monograph series. Health and the sociology of emotions (pp. 115-132). Malden: Blackwell Publishing.

Tsai, W., \& Ghoshal, S. (1998). Social Capital and Value Creation: The Role of Interim Networks. Academy of Management Journal, 41, 464-476. doi: $10.2307 / 257085$

Valderas, J. M., Starfield, B., Sibbald, B., Salisbury, C., \& Roland, M. (2009). Defining comorbidity: implications for understanding health and health services. The Annals of Family Medicine, 7(4), 357-363.

Valente, T. W., Corongesa, K. A., Stevens, G. D., \& , \& Cousineau, M. R. (2008). Collaboration and competition in a children's health initiative coalition: A network analysis. Evaluation and Program Planning, 31(4), 392-402. doi:doi: 10.1016/j.evalprogplan.2008.06.002

Van den Bulte, C., \& Lilien, G. L. (2001). Medical innovation revisited: Social contagion versus marketing effort. American Journal of Sociology, 106(5), 14091435. doi: 10.1086/320819

Vassilev, I., Rogers, A., Blickem, C., Brooks, H., Kapadia, D., Kennedy, A., .. Reeves, D. (2013). Social networks, the 'work'and work force of chronic illness selfmanagement: a survey analysis of personal communities. PloS one, 8(4), e59723.

Vincent, J. A. (2006). Age and Old Age. In G. Payne (Ed.), Social Divisions (Second editon). (Second ed., pp. 194-215). Basingstoke: Palgrave Macmillan.

Ward, V., House, A., \& Hamer, S. (2009). Knowledge brokering: the missing link in the evidence to action chain? Evidence $\mathcal{E}^{2}$ policy: a journal of research, debate and practice, 5(3), 267-279. 
Wäsche, H. (2015). Inter-Organizational Cooperation in Sport Tourism: A Social Network Analysis. Sports Management Review, 18, 542-554. doi:http://dx.doi.org/10.1016/j.smr.2015.01.003

Wasserman, S. a., \& Faust, K. (1994). Social Network Analysis: Methods and Applications. Cambridge: Cambrudge University Press.

Watts, T. (2016). Meeting the housing needs of older people: the key to better care and a more integrated society? Working with Older People, 20(4), 199-203.

Webb, C., \& Kevern, J. (2001). Focus groups as a research method: a critique of some aspects of their use in nursing research. Journal of advanced nursing, 33(6), 798805.

Welsh Assembly Government. (2000). In Safe Hands: Implementing Adult Protection Procedures in Wales. Cardiff Welsh Assemly Government Retrieved from http://www.wbsb.co.uk/pdf/In\%20Safe\%20Hands\%5B1\%5D.pdf.

Welsh Assembly Government. (2008). The Welsh Housing Quality Standard: Revised Guidance for Social Landlords on Interpretation and Acheivement of the Welsh Housing Quality Standard. In: Welsh Assembly Government.

Welsh Assembly Government. (2011). Sustainable Social Services for Wales: A Framework for Action. Retrieved from Cardiff:

Welsh, E. (2002). Dealing with data: Using NVivo in the qualitative data analysis process. Paper presented at the Forum Qualitative Sozialforschung/Forum: Qualitative Social Research.

Welsh Government. (2011). Together for Health: A Five Year Vision for the NHS in Wales. Retrieved from Cardiff: http://www.wales.nhs.uk/sitesplus/documents/829/togetherforhealth.pdf

Welsh Government. (2013). The Strategy for Older People in Wales 2013-2023. Retrieved from Cardiff: http://www.cpa.org.uk/cpa-lgaevidence/Merthyr_Tydfil_County_Borough_Council/The_Strategy_for_Ol der_People_in_Wales_2013-2023.pdf

Welsh Government. (2014a). A Framework for Delivering Integrated Health and Social Care For Older People with Complex Needs. Retrieved from Cardiff: https://gov.wales/docs/dhss/publications/140319integrationen.pdf

Welsh Government. (2014b). Help for the disabled and older people. Retrieved from http://gov.wales/topics/housing-and-regeneration/housingquality/maintenance-and-repair/disabledhelp/?lang=en

Welsh Government. (2014c). The Strategy for Older People in Wales. Retrieved from Cardiff:

http://gov.wales/topics/health/publications/socialcare/strategies/older/?l $\underline{\text { ang }=\text { en }}$

Welsh Government. (2014d). The Wales we want by 2050- A Welsh Society's commitment to a better quality of life for future generations. Retrieved from Cardiff: https://gov.wales/docs/dsjlg/publications/140218-wales-we-want-en.pdf

Welsh Government. (2014e). Welsh Indes of Multiple Deprivation (WIMD) 2014 Revised. Retrieved from Norwich, UK: http://gov.wales/docs/statistics/2015/150812-wimd-2014-revised-en.pdf

Welsh Government. (2014f). Welsh Index of Multiple Deprivation (WIMD) 2014revised. Retrieved from Norwich: http://gov.wales/docs/statistics/2015/150812-wimd-2014-revised-en.pdf

Welsh Government. (2015a). Well-being of Future Generations (Wales) Act 2015: The Essentials. Retrieved from Cardiff: http://gov.wales/docs/dsjlg/publications/150623-guide-to-the-fg-acten.pdf

Welsh Government. (2015b). Well-being of Future Generations (Wales) Act: The Essentials. Cardiff: Crown from 
http://gov.wales/docs/dsjlg/publications/150623-guide-to-the-fg-acten.pdf.

Welsh Government. (2017a). ENABLE-Support for Independent Living: A Better Way of Delivering Home Adaptations. In W. Government (Ed.): Crown.

Welsh Government. (2017b, 20-10-2017). National Population Projections. Retrieved from https://gov.wales/statistics-and-research/nationalpopulation-projections/?lang=en

Welsh Government. (2017c, 13th October 2017). Supporting People programme. Retrieved from https://gov.wales/topics/housing-andregeneration/services-and-support/supporting-people/?lang=en

Welsh Government. (2018, 25-06-2018). ENABLE-Support for Independent Living. Retrieved from https://gov.wales/topics/housing-andregeneration/housing-quality/aids-and-adaptations/support-forindependent-living/?lang=en

West, E., \& Barron, D. N. (2005). Social and geographical boundaries around senior nurse and physician leaders: an application of social network analysis. CJNR (Canadian Journal of Nursing Research), 37(3), 132-149.

West, E., Barron, D. N., Dowsett, J., \& Newton, J. N. (1999). Hierarchies and cliques in the social networks of health care professionals: implications for the design of dissemination strategies. Social science \& medicine, 48(5), 633-646.

Wey, T., Blumstein, D. T., Shen, W., \& Jordán, F. (2008). Social network analysis of animal behaviour: a promising tool for the study of sociality. Animal behaviour, $75(2), 333-344$.

Wharton, A. S. (1993). The affective consequences of service work: Managing emotions on the job. Work and occupations, 20(2), 205-232.

WHO. (2002). Proposed working definition of an older person in Africa for the MDS Project. Retrieved from http://www.who.int/healthinfo/survey/ageingdefnolder/en/

WHO. (2015). World report on ageing and health. Luxembourg World Health Organization.

Wiles, Crow, Heath, \& Charles. (2008). The management of confidentiality and anonymity in social research. International Journal of Social Research Methodology, 11(5), 417-428.

Wiles, Leibing, Guberman, Reeve, \& Allen. (2012). The meaning of "aging in place" to older people. The Gerontologist, 52(3), 357-366.

Williams, S. J. (1998). Capitalising'on emotions? Rethinking the inequalities in health debate. Sociology, 32(1), 121-139. doi: 10.1177/0038038598032001008

Winstanley, M. (2011, 17th February 2011). The Rural Exodus. BBC history. Retrieved from http://www.bbc.co.uk/history/british/victorians/exodus_01.shtml

Woodcraft, S. (2011). Mapping Social Networks to Improve Public Service Delivery: A Project with Neighbourhoos Management in King's Lynn, West Norfolk. In: The Young Foundation [online].

Woolcock, M., \& Narayan, D. (2000). Social capital: Implications for development theory, research, and policy. The World Bank Research Observer, 15(2), 225-249. doi: $10.1093 / \mathrm{wbro} / 15.2 .225$

Wright, R. W., Brand, R. A., Dunn, W., \& Spindler, K. P. (2007). How to write a systematic review. Clinical orthopaedics and related research, 455, 23-29.

$\mathrm{Xu}$, J., \& Chen, H. (2005). Criminal network analysis and visualization. Communications of the ACM, 48(6), 100-107.

Yang, S., Keller, F. B., \& Zheng, L. (2017). Social Network Analysis: Methods and Examples. London: Sage. 
Zapf, D., Vogt, C., Seifert, C., Mertini, H., \& Isic, A. (1999). Emotion work as a source of stress: The concept and development of an instrument. European Journal of work and organizational psychology, 8(3), 371-400.

Zhang, Yu, Fan, \& Duan. (2013). Research collaboration in health management research communities. BMC medical informatics and decision making, 13(1), 5265. doi:10.1186/1472-6947-13-52

Zhang, \& Zhang. (2009). Edge anonymity in social network graphs. Paper presented at the Computational Science and Engineering, 2009. CSE'09. International Conference on Social Computing, Vancover. 